\title{
AUTISM AND SOCIAL INTERACTION: A DISCURSIVE PSYCHOLOGICAL STUDY
}

\author{
BY \\ GATES ELEANOR HENDERSON
}

\begin{abstract}
A thesis
submitted to Victoria University of Wellington in fulfilment of the requirements for the degree of Doctor of Philosophy
\end{abstract}

Victoria University of Wellington

2020 


\begin{abstract}
In psychological research, autistic people are generally characterised as possessing disordered social cognition and embodiment in comparison to non-autistic people.

Specifically, a deficit in Theory of Mind (the capacity to think about other people's mental states in order to understand and predict their behaviour) and altered tactile sensation have been proposed as some significant psychological differences present in autism. Autistic people are characterised as experiencing social interactional difficulties that impact socialemotional reciprocity. Examples of such impact include struggling to approach others to interact or to make personal or relevant contributions to an interaction.

While there is a substantial literature on the cognitive properties of autistic individuals compared to non-autistic individuals and how these impact social psychological phenomena, there is considerably less research that analyses autistic people in their own right as social agents in naturally-occurring, everyday settings. As well, there is a challenge to the ideology behind deficit-oriented frameworks of autism in the form of the neurodiversity movement. This thesis draws on ethnomethodology, discursive psychology, and conversation analysis to contribute to both the naturalistic study of autistic people in social interaction and the development of positive, competence-oriented, and ecological approaches to autism. This will be achieved by analysing the social action, as produced in talk and with the body, of autistic children in interaction with their family members in their homes.
\end{abstract}

Ten hours of video recordings were collected in the homes of four volunteer families with at least one autistic child member. Recordings were made by the families themselves of the mundane domestic activities they engaged in, including episodes of cooking and mealtimes, members playing together, preparing for school, and discussing the day's activities. After detailed transcription, instances of the children providing accounts for their own behaviour and embraces (or resistance to them) were collected for and became the focus of detailed analysis. An extended sequence constituting a common parenting activity (directing a child to do something) was also selected. This research takes the domains of Theory of Mind and tactile sensation that are prominent within psychological research on autism and treats them as social interactional accomplishments.

The first empirical chapter examines how children accounted for their own behaviour. It found that the children's accounts were oriented toward the displayed expectancies and characterisations of the child and their conduct either in responding to first pair parts (e.g., 
resisting a suggestions with an embedded presumption of the child's knowledge), or in launching their own first action (e.g., requesting more food). These accounts constitute concern for how the children's interactants could, or do, treat them in response to their behaviour, accomplishing Theory of Mind embedded in their everyday action.

With respect to tactile sensation, the second empirical chapter analyses embraces. Embraces occurred within and between a variety of other activities. Analyses showed how both children and parents initiated embraces and many were accomplished as nonproblematic by the children. Participants arranged their bodies such that the embrace was coordinated with the talk and ongoing action, and utilised both verbal and embodied resources to initiate and terminate. Children prioritised their ongoing actions, treating some embraces or embrace initiations as interruptive by avoiding, escaping or otherwise misaligning with them.

The third empirical chapter demonstrates how one family's extended sequence of action directing their child to use the bathroom before bedtime was comprised of a variety of different relational activities. In the process of managing the larger project of the directive, parent and child negotiated complex elements of their relationship including issues of power and responsibility, shared knowledge and experiences, and expectations of group membership.

This thesis offers a critical perspective on the conceptualisation of autism in psychology. It grounds this alternative view of autism based on an empirical analysis of how the autistic children and their family members in the interactions analysed manage complex social psychological matters in the production of their social action. It expands upon discursive psychological research on the accomplishment of social cognition as action produced within talk-in-interaction. It also exemplifies a direction a neurodiversity-sensitive psychology of social action could take and identifies ways that this can be further developed. 


\section{Acknowledgements}

Working on my PhD research has been one of the most enjoyable and rewarding times in my life. The research that I have done, the topics I have engaged with, and the people who have appreciated my work have provided me with a sense of meaning, value, and understanding of things that I didn't have before. I'm going to thank a few specific people here but note that if you have interacted me at any point through this I appreciate you and your patience as I probably talked to you ad infinitum about this work.

First, I want to acknowledge the families that participated in this research. I'm so grateful that you all have allowed me into you homes to record this data. Your participation has meant the world to me and without it this research could not have happened. Thank you so much.

I am very thankful to have had the support of my supervisor, Ann Weatherall. Since discovering conversation analysis from you those years ago, our relationship has been very important to me. Your passion for the research has rubbed off on me and that, along with your concern for my wellbeing and future, has shown me so much of what I would want to be as a supervisor myself. Thank you for encouraging me to investigate the diverse topics I have in this process, encouraging me to engage with literature outside our psychological box, and broadening the horizons of my thinking.

I am intensely grateful to have been able to share these last few years, and an office, with my Studies in Social Interaction comrades Emma Tennent and Fiona Grattan. You two have been immensely helpful in encouraging my confidence in my analytic work as well as providing stability to my otherwise flighty nature. All the conversations we have had, the ideas and values we have shared have helped more than most to get me to where I am today as an academic (and a person more generally). I'm excited for our futures as dream team collaborators.

While I haven't shared an office with her for very long, Jessica La has also been a much appreciated on my support team. I feel like we are similar in a lot of ways and that we are pretty good at hyping each other up in terms of confidence and saying our piece. Always love to hear your points of view on things.

We have had so many people through the Studies in Social Interaction research group over the last few years. I won't name you all for fear of missing someone out but having you 
all at data sessions over the years and allowing me to tell you everything and more about what I'm doing has all helped. And having a strong team has been an inspiration for me.

I'm thankful to have had such a supportive group of friends in the postgrad cohort. Michaela Pettie, Gloria Fraser, Sophie Hedley, Kealagh Robinson, Kris Nielsen, Jess Shaw, Kate McLeod - I appreciate that you have all encouraged me and supported me (despite my EMCA fanaticism) and, especially, that you have all stuck with me when I've not been particularly good at reaching out. Knowing that you are all there means a lot to me and I hope to all continue in the form of QuACC for a while yet.

As with the above, I need to thank all my non-psychology friends for sticking with me despite it being very difficult to leave my psychology bubble. Amber Bohanna, Joseph Habgood, Adeline Greig, Adam Crawshaw, Jaime Grace, Britnee Tapara - Being there when I needed to talk, and letting me talk your ears off has been more help than you could imagine. You have all supported me in the troubles and joys of doing this work.

I am very grateful to Mum who has practically provided for me when things got tough and always been a strong supportive voice. Even though so much of our communication has been about my work and university issues, it's always nice to know you're there to hear it. And I love hearing that you brag about me to people. Always good for a confidence boost.

Lastly, I want to recognise Victoria University of Wellington and the financial support they've provided in the form of the Victoria Doctoral Scholarship, without which I could not have done this work. 


\section{Contents}

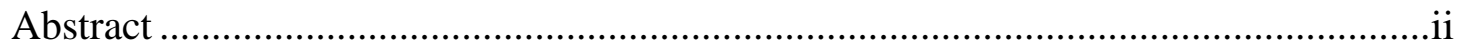

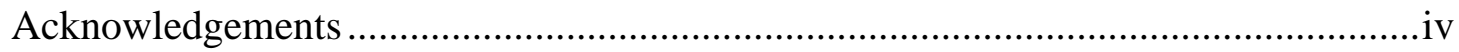

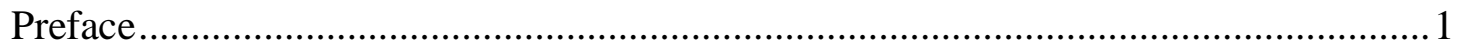

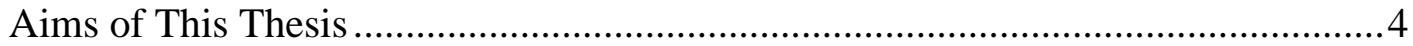

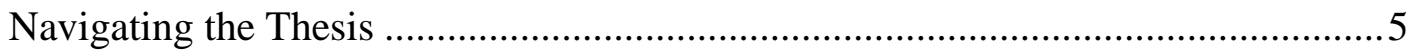

Chapter One: Neurodiversity and the Study of Social Interaction..............................

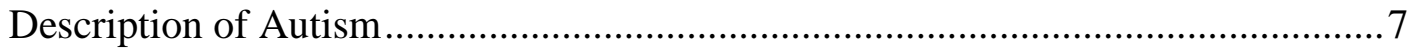

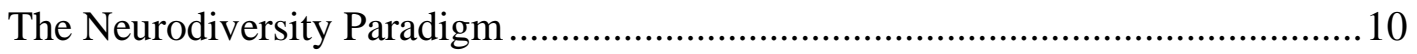

Ethnomethodology and Respecifying the Social ................................................. 13

Conversation Analysis and Studying Talk-in-Interaction.................................... 17

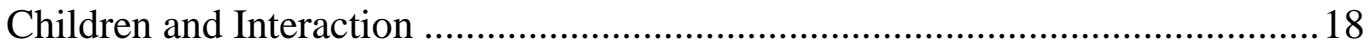

Accomplishing the Psychological: Discursive Psychology ....................................20

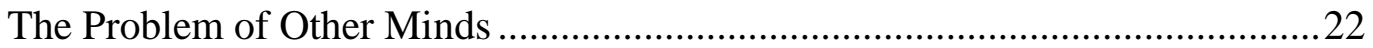

Mind-Reading? Or, Social Practice? ...................................................................2 23

Discursive Psychology for Critical Autism Studies...............................................26

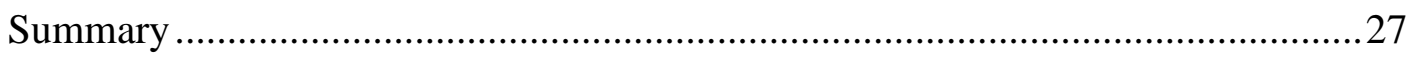

Chapter Two: Autism, Language and Social interaction ...........................................29

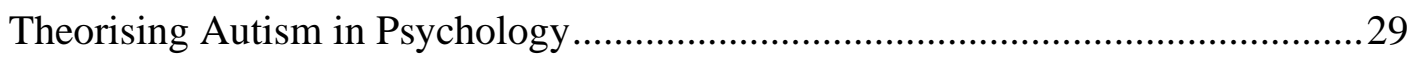

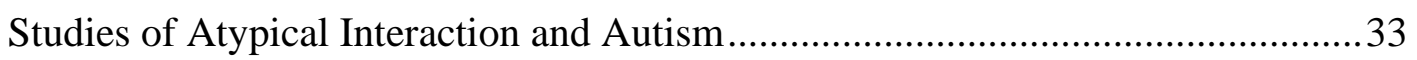

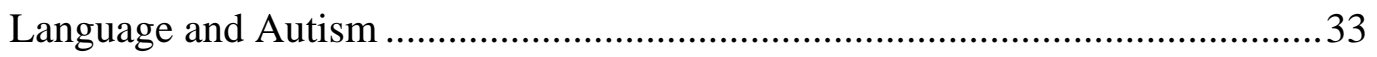

Naturalistic and Conversation Analytic Studies of Autism ................................ 35

Methodological Fusion: Conversation Analysis and Experimental Psychology.38

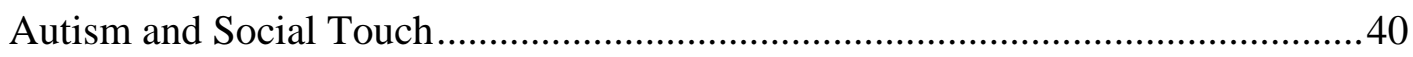

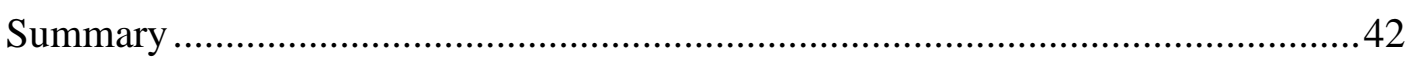

Chapter Three: Doing Discursive Psychology: Data Collection, Transcription and

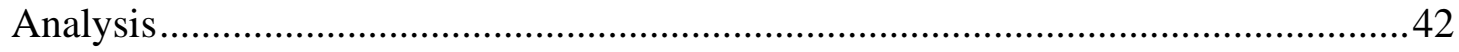

Observation and Naturalistic Data in Social Sciences ............................................43

Considerations Made in Collecting Naturalistic Video Data................................... 45

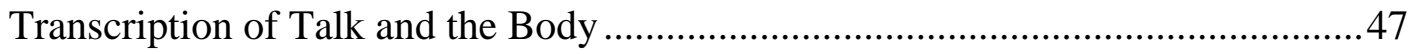

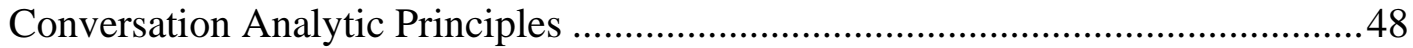

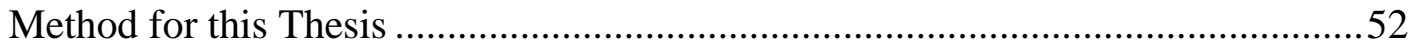

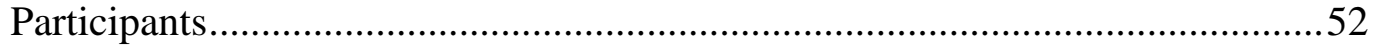

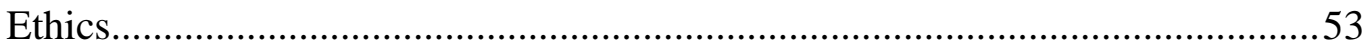

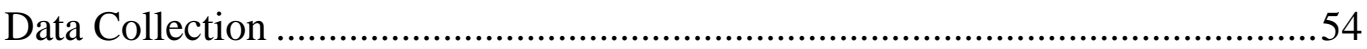




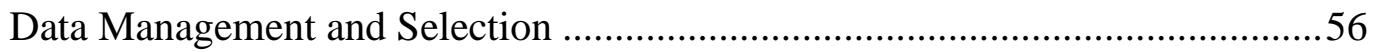

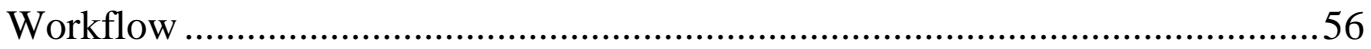

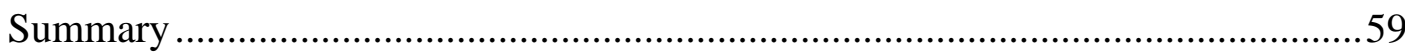

Chapter Four: Enacting Self- and Other-Orientation by Providing Explanations of

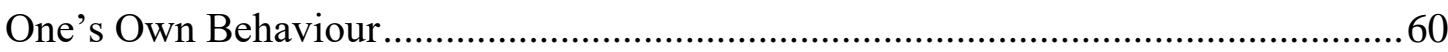

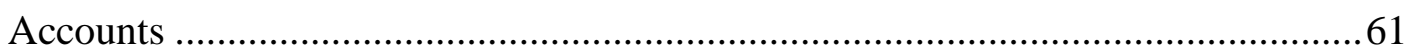

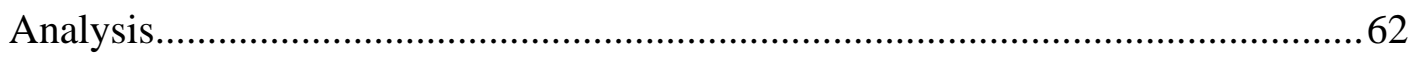

Explanations Constructing the Link between Cognition and Conduct ................63

Explanations Addressing Others' Understandings Displayed in First Pair Parts 65

Explanations Deployed in Initial Position .......................................................69

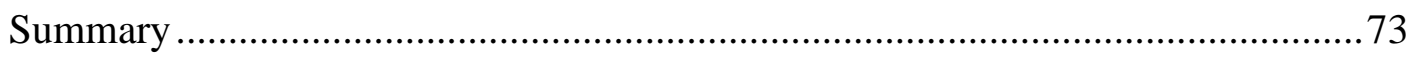

Chapter Five: Approach and Avoidance of Affectionate Touch by Autistic Children 75

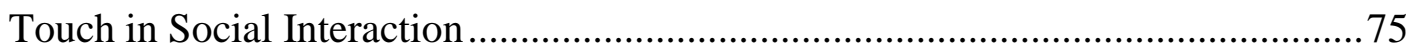

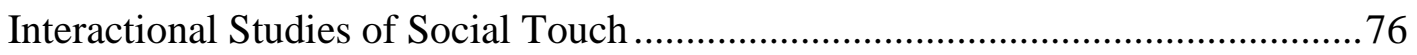

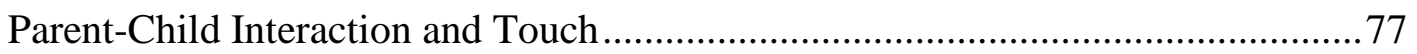

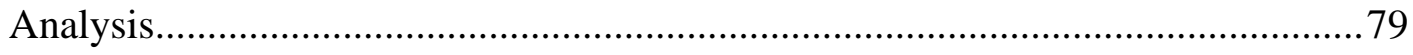

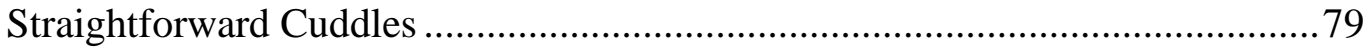

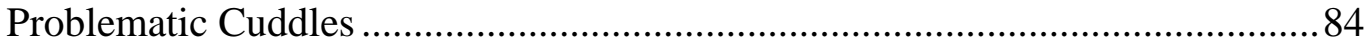

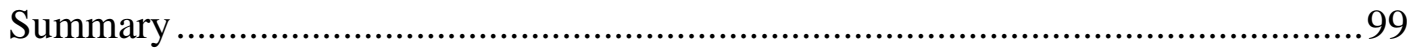

Chapter Six: Negotiating an Activity Contract Between an Autistic Child and their

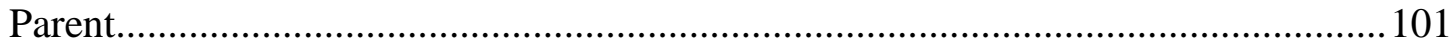

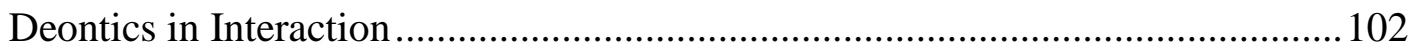

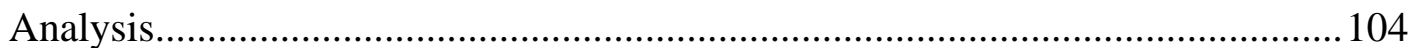

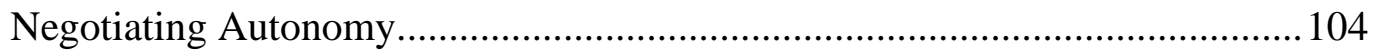

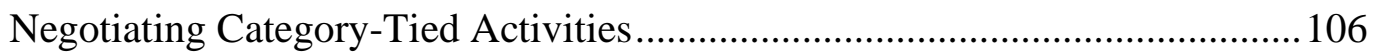

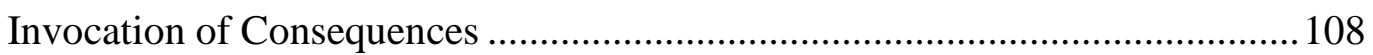

Utilising Beneficiary Status and Coercion......................................................... 111

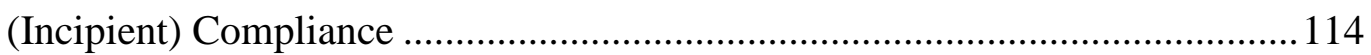

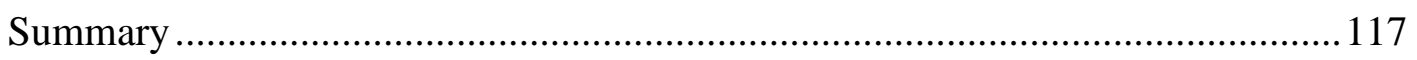

Chapter Seven: Respecifying Autism: General Discussion ..................................... 119

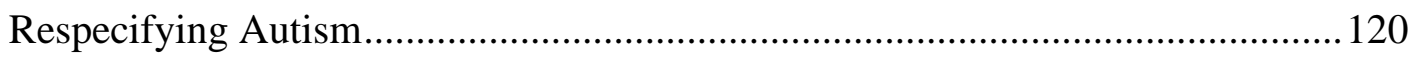

Accounts as Complex Orientation to Others in Interaction ............................. 121

Engaging in, and Avoiding, Social Touch in Interaction................................. 124

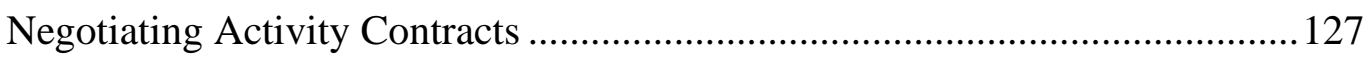

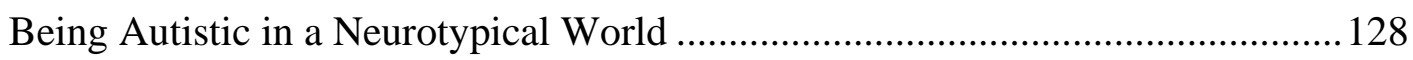


The Future for Discursive Psychology and Neurodiversity Studies ..................... 132

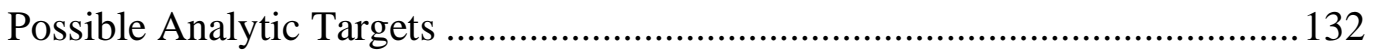

Cognition, Behaviour, and Masking …......................................................... 133

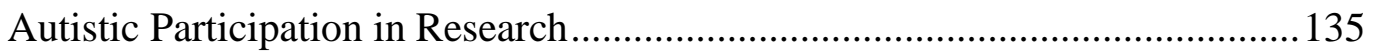

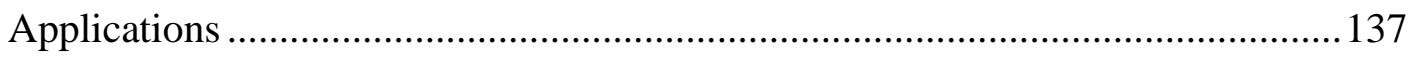

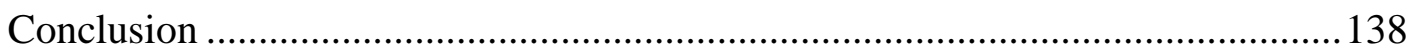

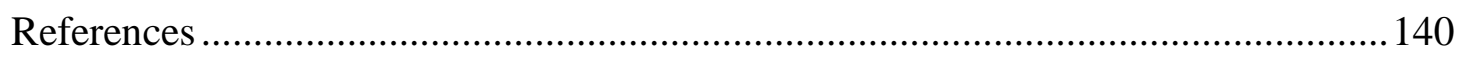

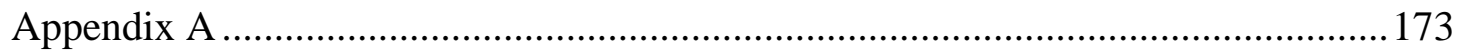




\section{Preface}

"There is from the start an extreme autistic aloneness."

(Kanner, 1943, p. 242)

Leo Kanner (1943) first described infantile autism and its associated social deficits after clinical observations of children. He noted that they displayed an extreme disinterest in the external world and other people, anxious attempts at the "maintenance of sameness" (p. 245), and a limited behavioural repertoire. Infantile autism is now known by its modern, formal diagnostic title Autism Spectrum Disorder, or simply autism. Research on autism and autistic people ${ }^{1}$ has tended to perpetuate the idea that it is pathological and based on disordered, or deficient cognitive processing. This thesis contributes to a social and academic movement to challenge this tendency by understanding autism as an example of natural human diversity in thinking and acting and analysing autistic children's naturally occurring social interaction to demonstrate their sophisticated (yet possibly different) social ability.

Table 1

Illustrative Behaviours Constituting ASD Diagnostic Criteria

\begin{tabular}{|c|c|}
\hline $\begin{array}{l}\text { Social communication and interaction } \\
\text { deficits }\end{array}$ & Repetitive, stereotyped behaviours \\
\hline $\begin{array}{ll} & \text { Little initiation of interaction } \\
- & \text { No emotional reciprocity } \\
- & \text { Reduced imitation of others } \\
- & \text { Tendency to label or request over } \\
& \text { contributing to conversation } \\
- & \text { Atypical use of non-verbal } \\
& \text { communication (e.g., eye contact, } \\
& \text { gesture, facial expression) } \\
- & \text { Reduced social interest } \\
- & \text { Lack of social play and imagination } \\
- & \text { One-sided friendships }\end{array}$ & $\begin{array}{ll}\text { - } & \text { Motor stereotypies (i.e., hand } \\
& \text { flapping) } \\
- & \text { Repetitive speech } \\
\text { - } & \text { Excessive concern with ritual or } \\
\text { routine } & \text { Hyper- or hypo-reactivity to sensory } \\
\text { - } & \text { input } \\
\text { - Excessive sensory restrictions (i.e., } \\
\text { with regard to food) } \\
\text { - Excessive fascination with specific } \\
\text { domains of interest, objects, or } \\
\text { sensations }\end{array}$ \\
\hline
\end{tabular}

Note: list drawn from the DSM-5 (American Psychiatric Association, 2013).

Autism spectrum disorder (ASD, or autism) is characterised in the Diagnostic and Statistical Manual of Mental Disorders (5th ed.; DSM-5; American Psychiatric Association, 2013) as a neurodevelopmental disorder characterised by difficulties in social communication

\footnotetext{
${ }^{1}$ On the basis of Kenny et al., (2015), identity-first language (e.g., autistic person, as opposed to person with autism) will be used in this thesis.
} 
and social interaction as well as by repetitive, stereotyped behaviours. Behaviours that contribute to the meeting of these criteria are described below in Table 1 .

In New Zealand, an Autism Spectrum Disorder diagnosis is ideally given by a multidisciplinary team comprised of members from different professions including, paediatricians, psychiatrists, psychologists, and speech-language therapists (New Zealand Ministries of Health and Education, 2016). The core components of the diagnostic process include; interviews with the person being assessed as well as with relevant others like parents/family members, or teachers; observations of the individual in both structured and unstructured settings; evaluation of medical history; and assessments of communication and cognitive functioning (New Zealand Ministries of Health and Education, 2016). These components are comprised of, or supplemented as needed by, standardised assessment measures, including rating scales (like the Childhood Autism Rating Scale; CARS: Schopler, Reichler, \& Renner, 1986), semi-/structured interviews (like the Autism Diagnostic Interview-Revised; ADI-R; Le Couteur, Lord, \& Rutter, 2003), and standardised observation schedules (Like the Autism Diagnostic Observation Schedule, $2^{\text {nd }}$ Edition; ADOS-2: Lord, Rutter, DiLavore, Risi, Gotham, \& Bishop, 2012). There are no biological markers of autism and all assessments and diagnoses of Autism Spectrum Disorder depend on clinicians' judgements (Karim, 2017).

Diagnoses of autism have increased in prevalence significantly since its first description. In the past, the prevalence of autism has been reported as 1 in 10000 people (Karim, 2017), however recent estimates by the Center for Disease Control (Maenner et al., 2020) has been as high as 1 in 54 (1.9\%) people having a diagnosis of Autism Spectrum Disorder. The New Zealand Ministries of Health and Education (2016) suggest that there were, as of their writing, at least 40,000 autistic individuals in New Zealand. This increase in prevalence rate has been controversial. While some consider it the result of an actual rise in prevalence due to things like vaccines, evidence suggests it is the result of broadening the diagnostic category (Gernsbacher, Dawson, \& Goldsmith, 2005), and increased awareness of autism by parents, professionals and self-advocates (Eyal, 2013). In studies from the United Kingdom, children tended to be diagnosed with Autism Spectrum Disorder after entry into school (Hosozawa, et al., 2020) and problems in schooling, as well as in social development, language delays, obsessive and problematic behaviour were the kinds of things parents tended to report when first accessing assessments and support for their children later receiving an autism diagnosis (Crane, Chester, Goddard, Henry, \& Hill, 2015). 
Autism is considered a highly heterogenous condition. As well as the behavioural characteristics captured by the diagnostic criteria, autistic children are reported to commonly experience other conditions such as attention-deficit/hyperactivity disorder, mood disorders, sleep disorders, language disorders, food related difficulties, aggression or difficult temperament, and epilepsy (Mannion, Leader, \& Healy, 2013; Soke, Maenner, Christensen, Kurzius-Spencer, \& Schieve, 2018). As well, while autistic people's overall quality of life is generally positive (when studied generally and by self-report; Moss, Mandy, \& Howlin, 2017), individual aspects of quality of life are commonly more negative than that of nonautistic people (van Heijst, \& Geurts, 2014).

A particular aspect of quality of life that is impacted in autistic people's lives is the degree to which they engage in social activities with other people or participate in social settings. Parent-rated conversational ability and the capacity to perform certain daily functional tasks (e.g., using public transportation, make trip arrangements, read an analog clock) are negatively correlated with social participation (Orsmond, Shattuck, Cooper, Sterzing, \& Anderson, 2014) and autistic people report difficulties interacting with nonautistic people (Crompton, Hallett, Ropar, Flynn, \& Fletcher-Watson, 2020). As well as that, negative stereotypes of autistic people are still common (Wood, \& Freeth, 2016) and nonautistic people are less likely to interact with autistic people based on first impressions (Sasson, et al., 2017; though this tendency is mitigated with diagnosis disclosure; Sasson, \& Morrison, 2017). As such, a big part of autistic people's social quality of life is shaped by the impacts of a world not designed for them, as well as non-autistic people's tendency to find autistic people difficult to understand (Alkhaldi, Sheppard, \& Mitchel, 2019) and their stigmatising beliefs (Morrison, DeBrabander, Faso, \& Sasson, 2019).

While these stereotypes and stigmas are still widely endorsed, research tends to focus on presumed deficits in autistic social ability and cognition. Globally, funding for autism research prioritises biological research and assessments of interventions (den Houting, \& Pellicano, 2019; Pellicano, Dinsmore, Charman, 2014). However, there is a move to address this by critiquing old narratives about autism and autistic people and constructing new ones. This effort at critical autism studies is couched within the neurodiversity paradigm, as is the research presented in this thesis. Neurodiversity paradigm refers to an approach to neurodiversity (i.e., the diversity of brain and cognition with humanity) that recognises such diversity as natural and challenges the status of different patterns of neurocognitive functioning as healthier than others (Walker, 2014). Within this paradigm, autism is 
recognised as a difference in psychological functioning compared to the neurotypical norm. Autism is not in itself disordered or deficient but can appear as such in dominantly neurotypical social spaces.

This thesis will demonstrate a way of progressing neurodiversity paradigm research and understanding autism/autistic people by studying autistic people's social behaviour in situ. This will be accomplished by adopting a discursive psychological approach, informed by ethnomethodology, to study autistic children interactions with their family members.

\section{Aims of This Thesis}

This thesis critically examines, using conversation analysis within a discursive psychological framework, three phenomena in autistic children's social interaction. These phenomena are; the provision of explanations for their own behaviours; the engagement in, and avoidance of, physical affection; and the negotiation of an activity contract between a child and their parent.

These phenomena concern the development and recognition of interpersonal relationships and who participants are to each other, the management of other people's knowledge and specifically their understanding of what the autistic individual is doing, and the practical management of social touch. As such, they constitute central phenomena in the domains typically considered sites of disorder in autistic people's social interaction. This thesis aims to describe how the participants in this research navigated these sites together in order to co-produce a coherent social landscape, critiquing the common psychological position that autistic individuals are, by definition, socially disordered. This thesis is not concerned with whether the autistic participants manage each of these interactional practicalities well or in a manner resembling how neurotypical interactants do. What is of concern is how these kinds of practicalities are managed by the autistic individuals and their interactional co-participants when they arise.

As well as critique and building a positive image of autistic sociality within the family, this thesis speaks to broader debates around what it means to be a social actor. Drawing on discursive psychology, this thesis contributes to broader discussions on cognition, experience, and action. In analysing how these autistic children and their families navigate the social world themselves, this thesis also documents the ways that they solve interactional problems that are common to all social actors; how do we make ourselves understandable by other people?; how do we make our experiences available and 
recognisable to others?; how do we draw on the features of our local interactional world to progress or resist activities and the ways those activities construct our identities? In addressing these more general social questions, this thesis will show how these can be managed both by participants and studied by analysts without reference to the mind or cognition.

\section{Navigating the Thesis}

This thesis is divided into eight chapters. Chapter One introduces the key frameworks and methods used in this thesis to approach the study of autistic people's social interaction. The neurodiversity paradigm will be discussed before introducing ethnomethodology as an approach to the social inquiry that accomplishes some of what the neurodiversity movement and critical autism studies aims to do. An example of what ethnomethodology and conversation analysis can do for neurodiversity and critical autism studies will be given by showing what these approaches have done for the study of childhood and children's social behaviour. Discursive psychology, as an approach to psychological inquiry that draws on ethnomethodology and uses conversation analysis will be introduced next and discussed in terms of how it addresses psychological concepts that are considered critical to autism (e.g., Theory of Mind).

Chapter Two presents a review of research literature on autism language and social interaction. Descriptions of autism have centred social ability, but modern theories have tended to present largely asocial cognitive causes. Some of these core theories will be introduced before a discussion of research broadly from the domains of clinical linguistics and psychology that has investigated differences in autistic people's talk compared to neurotypical talk. Research drawing from conversation analysis will be reviewed next, showing how a focus on social interaction in situ highlights the accomplishment of social action by autistic people in interaction with others. Finally, recent research on autism and touch will be reviewed, to be picked up again specifically in Chapter Five.

Chapter Three will discuss the data and methods of analysis. Theoretical and methodological considerations to be made in designing research involving naturalistic data will reviewed before I describe the particular methods used in this research.

Chapters Four, Five, and Six comprise the analytic segments of the thesis. Chapter Four provides an analysis of autistic children's explanations for their own behaviour, discussing primarily how they are designed with an orientation to others knowledge and 
presumptions about their behaviour. Chapter Five provides an analysis of moments of social touch (specifically embraces) as they occur in the course of social interaction. The focus will be on how both parents and children initiate embraces and how the children resist or abandon embraces and what this might suggest about autistic people's social touch aversion and the study of it. Chapter Six provides an extended case study looking at how a mother directs her child and how the child resists this direction. This analysis demonstrates the online development, maintenance, and transformation of the relationship between the child and their mother.

Chapter Seven will summarise and synthesis the findings of the individual analytic chapters and draw them together in a discussion of what these studies can tell us about autistic people's social interaction and how psychologists should approach research in this area. I will argue for a respecification of autism and explain how the findings of this thesis align with that respecification. I will also discuss the ways in which discursive psychological research like that presented here can develop in approaching autism and autism-relevant psychological concerns, both in terms of specific targets for analysis and involvement of autistic participant researchers. 


\section{Chapter One: \\ Neurodiversity and the Study of Social Interaction}

"...counter the imaginary ideal of a cognitive normal subject."

Bertilsdotter Rosqvist, Stenning, \& Chown (2020, p. 228)

Bertilsdotter Rosqvist, Stenning, and Chown said the above in the context of describing the epistemological positions of the chapter authors within the recent text Neurodiversity Studies (2020). This thesis means to contribute to this counter. In this chapter, I will briefly describe autism as it is generally understood in psychology and psychiatry. I will then introduce the position of the neurodiversity paradigm (Walker, 2014). This critical paradigm challenges the conceptualisations of autism and autistic people popular within psychological and medical work by positioning the features commonly ascribed to autism as equally a part of humanity's psychological diversity. Following that, ethnomethodology, conversation analysis, and discursive psychology will be introduced as approaches to studying the social and the psychological that prioritise the actual, real-life accomplishment of action. These approaches will be characterised in ways that demonstrate how they align with the critical agenda of the neurodiversity paradigm and critical autism studies in particular by centring autistic people's lived activity and reframing social difficulties autistic people have into interactional problems to be solved by all parties.

\section{Description of Autism}

Autism is more fully named Autism Spectrum Disorder in the DSM-5 (American Psychiatric Association, 2013). Autism is characterised as a neurodevelopmental disorder. Neurodevelopmental disorders are conditions in which the brain develops in such a way that it functions and responds to stimuli in ways that are substantially different from typically developed brains (Karim, 2017) with their initial onset in early development. Historically, the formal features of Autistic Spectrum Disorder ${ }^{2}$ were described as the triad of impairments. The triad consisted of impairments to social interaction, communication ${ }^{3}$, and imagination

\footnotetext{
${ }^{2}$ Note that in its initial DSM form, autism was referred to as the autistic spectrum, not the autism spectrum (Wing, 1981a; Wing, \& Gould, 1979)

${ }^{3}$ The distinction between social interaction and communication made here is generally a distinction between understanding of norms at play in social settings versus production and comprehension of language.
} 
(Wing, 1981a; 1981b). Since the publication of the DSM-5, the diagnostic criteria for Autism Spectrum Disorder have condensed this triad into two criteria. The first criterion is deficits ${ }^{4}$ in language and social communication. These deficits might be characterised by reduced social-emotional reciprocity in social interaction with others, difficulties reading or using non-verbal communicative actions, and difficulties with developing and maintaining relationships (American Psychiatric Association, 2013). The second criterion is "restricted, repetitive patterns of behavior, interests, or activities" (p. 50) as demonstrated by at least two of; repetitive movements/behaviours (like repeating speech of hand flapping); preference for routines and structure; highly specific interests; and either heightened or lowered sensory reactivity to sensory input.

Autism appears in people young and is persistent throughout the lifespan. Evidence suggests that when autism is diagnosed it is most commonly done so around the age of entry into school (Hosozawa et al., 2020), but recent research suggests that diagnosis can be relatively confidently made around 14 months of age (Pierce et al., 2019). Recent estimates by the Center for Disease Control (Maenner et al., 2020) suggest a prevalence rate of autism around 1 in 54 (1.9\%) people while in New Zealand the estimate is around at least 40,000 autistic individuals (New Zealand Ministries of Health and Education, 2016).

As autism is generally considered a neurodevelopmental disorder, there has been considerable research conducted in order to understand what causes as well as whether (and how) it can be treated. While no absolute causal links have been made (Hodges, Fealko, \& Soares, 2020), researchers have reported a large variety of both postnatal and prenatal factors that are associated with autism. Autism is thought to have a substantial genetic on the basis of studies comparing prevalence rates in monozygotic and dizygotic twins (Hallmayer et al., 2011; Rosenberg et al., 2009). For example, in a study of 277 twin pairs, the concordance rate for monozygotic twins (i.e., the rate at which both twins would receive an autism diagnosis) was $31 \%$ and for dizygotic twins $88 \%$, suggesting strong, heritable genetic factors (Rosenberg et al., 2009). Within families there is also what is called the broader autism phenotype. This is a tendency for family members of someone diagnosed with autism to display autism-like behaviours (Pickles et al., 2000). Many potential underlying genetic factors behind this heritability have been studied, finding a massive diversity of possible contributions to neuronal differences (Geschwind, 2011).

\footnotetext{
${ }^{4}$ Deficit is the term used in the DSM-5.
} 
Chemical differences in the brains of autistic people (compared to non-autistic people) have been found. Neurochemically, most major neurotransmitter systems studied have been found to differing between autistic and non-autistic people's brains. For example, the serotonin system appears to be comparatively different in autistic people's brains. Autistic people often have higher levels of blood serotonin compared to non-autistic people and this is thought to be related to the different function of the serotonin reuptake transporter (Cook Jr et al., 1997). A gene which regulates the function of the serotonin transporter has been linked to both high blood serotonin and altered social, as well as repetitive, behaviour (VeenstraVanderWeele et al., 2012) and some autistic people appear to demonstrate social behaviour more similar to non-autistic people under treatment with selective serotonin reuptake inhibitors (Moore, Eichner, \& Jones, 2004). As well, multiple aspects of the dopaminergic system (synthesis, reception, and reuptake) have been found to differ in autistic people's brains (Nguyen et al., 2014), and this has been linked to repetitive and stereotyped behaviour by autistic people (Staal, 2015).

Autistic people's brains are argued to be both structurally and functionally different from non-autistic people's brains as well. Researchers argue that autistic people's brains possess a different degree of connectivity, with aspects of hyper- and hypoconnectivity found compared to non-autistic people's brains (Di Martino et al., 2013; O'Reilly, Lewis, \& Elsabbagh, 2013). As well, specific brain regions are implicated in autism. For example, the size of the amygdala has been seen to correlate with the degree of social difficulties demonstrated in autistic people's brains (Juranek et al., 2006). Functional magnetic resonance imaging studies have shown functional differences in many different regions of the brain, including regions typically thought to be involved with face perception, perspective taking, and communication (Dichter, 2012).

Autism is described in the DSM-5 as a neurodevelopmental disorder. As a result the focus on research has tended to be on the individual-level correlates (e.g., genetics, neurology, behaviour) of the individual so diagnosed. As well, these correlates and features of autism are commonly described in relation to a presumed normal thinking and acting human, leading autism to be described in terms of reductions from or deficits of the normal capacity. This approach is challenged by the neurodiversity movement, described below. 


\section{The Neurodiversity Paradigm}

As discussed above, autism is characterised by the DSM as a disorder of cognitive and neurological development. However, a movement has developed, both within academic and activist circles, to challenge this characterisation by emphasising neurodiversity. Neurodivergence refers to the "perceived variations seen in cognitive, affectual, and sensory functioning differing from the majority of the general population" (Rosqvist, Stenning, \& Chown, 2020, p. 1). Nick Walker (a neurodiversity scholar) proposed the terms pathology paradigm and neurodiversity paradigm to refer to two distinct approaches to differences in psychological and behavioural functioning. While the pathology paradigm emphasises deficiencies, disorder, and difference as a contrast against health (and is the dominant conceptual framework for understanding psychological and behavioural diversity), the neurodiversity paradigm recognises that neurodiversity is natural and valuable, and that characterisations of this diversity as disordered is a construction that relies on dynamics of power and inequalities in society (Walker; 2013; Walker, 2014; Chapman, 2019).

The neurodiversity movement is concerned with criticising the "default pathologization and undue medicalization of "natural human variants"' (Chapman, 2019, p. 371) by psychiatry, psychology, and society more generally. It does this by drawing inspiration from social models of disability (Oliver, 1990). Social models of disability make a distinction between impairment and disability (Oliver, 1990, Goodley, 2011). Impairment is generally thought of in terms of a physical or mental limitation that impedes some kind of action (e.g., inability to walk due to paraplegia, or difficulty reading certain text like in dyslexia), and disability is the social and political consequences of that impairment (like not being able to enter buildings without ramps, or to read signs because texts are dominantly in fonts unreadable by dyslexic people). This distinction highlights that differences in ability are not necessarily natural and are often products of society's organisation.

The neurodiversity movement aims to deconstruct the presumed inherent disability in conditions like autism, to "problematise neurotypical domination" (Bertilsdotter Rosqvist, Stenning, \& Chown, 2020, p. 2) and show instead how many of the disabling features of these conditions are the result of their participation in a society that does not cater to their unique ways of engaging with the world, or is in fact actively hostile to them. An example of this regarding autism is the fact that autistic individuals commonly express heightened sensory sensitivity (Kern et al., 2006). This sensitivity itself is not necessarily disabling or disordered, but in combination with what society as a whole deems appropriate sensory stimuli in public life (e.g., the harsh lighting and music played in supermarkets or malls, or 
the general preference of people to wear scented deodorants and perfumes) autistic people's sensory hyperresponsivity can be disruptive and disabling.

An important feature of the neurodiversity paradigm is the recognition that the conditions hitherto described are produced by, and exist within, a broader cultural context (Nadesan, 2005). Not only are neurodivergent people made disabled in their interactions with a dominantly neurotypical society, the categories we ascribe membership of disabled people to are produced and reproduced in those interactions too. Philosopher Ian Hacking proposed that conditions like autism are not to be thought of as natural kinds to be objectively described as biologically or physically real by medical and psychological professionals. Instead, he suggests they be thought of as human kinds developed, maintained, and altered through a complex process of interactions. These interactions are between the so categorised individuals, the professionals that study and serve those individuals, and the broader society in which those category memberships are consequential (Hacking, 1996; 2006). When considered in these terms, autism appears not as a distinct biological entity which itself causes harm to those it affects, but instead as a constructed socio-political category deployed to address a population with certain dispositional tendencies.

The neurodiversity movement as a social movement encourages a change in how autistic people are seen as being made disabled by society, and therefore implies required changes for society's organisation such that the disabling is mitigated or removed entirely. However, the neurodiversity paradigm does not just impact our ethics and societal concerns; it is also scientifically advantageous to adopt a diversity approach (compared to a deficit approach) for studying how autism actually plays out in the world. Dinishak (2016) explains that deficit understandings of autism impede scientific progress in studying autism and autistic people in two significant ways. First, describing something like autism only in terms of what it is not produces only a negative characterisation of autism, leaving autism with no positive identity. Second, because deficits are a comparative quantitative concern, deficit thinking only cares for those features of autism which can be counted in all individuals for comparison, ignoring the possibility that there may be important qualitative differences or similarities which would not be found by measurement.

On the basis of these criticisms, the neurodiversity movement adopts stances towards research similar to the stances taken by many feminist researchers in studies of gender. For example, Nick Walker (2013) drew on feminist Audre Lorde when referencing her famous quote "For the master's tools will never dismantle the master's house" (Lorde, 1984, p. 110- 
114), recognising that without shifting paradigms, approaches, and methods, research is doomed to reinforce the system of oppression that research into autism has been a major part of.

The neurodiversity paradigm serves as both an ethical approach to the societal treatment of autistic people and as an academic approach to the study of autism in the world. Researchers working within the neurodiversity paradigm have founded the nascent field of critical autism studies. Critical autism studies is an autistic-led field of research with the aims of critically "investigating power dynamics that operate in discourses around autism, questioning deficit-based definitions of autism, and being willing to consider the ways in which biology and culture intersect to produce 'disability"' (Waltz, 2014, p. 1337; Woods, Milton, Arnold, \& Graby, 2018). Using "inclusive and non-reductive methods" (Orsini, \& Davidson, 2013, p. 12), critical autism studies are intended to provide new, emancipatory theories on autism, it's culture, and the relationship between autism, biology and society (Orsini, \& Davidson, 2013; Chown, et al., 2016).

This shift in paradigm has resulted in new characterisations of what autism is, and what it means to be autistic. Different accounts have arisen to shift focus in autism research away from considering autistic people as disordered individuals and towards considering autistic people as participants in a broader environment in which autism manifests (Milton, 2013; Chapman, in press). An important piece of this reorientation has been the recognition of the double empathy problem (Milton, 2012). Autism has traditionally been positioned as the reason why autistic people cannot or do not engage appropriately in social interaction with others as well as the reason why others cannot understand them or engage with them appropriately. The double empathy problem is a reconsideration of the problem of social interaction between autistic and non-autistic people where both sides experience difficulty in appropriate social interaction. This is not due to the autistic persons lack of typical social ability but due to the fact that autistic people and non-autistic people engage in sociality differently. In this regard, what might be called autism is less an internal feature of the solabelled autistic person but is more a feature of "the social interaction between two differently disposed social actors" (Milton, 2012, p. 884).

As the double empathy problem notion shows, much of what is considered autism actually lies in the interactional space between actors, rather than within individually disordered people. Some researchers have taken this notion further to suggest that autism as a 
whole does not exist as a biomedical entity, but as a constructed, historically-, socially-, and politically-contingent category within which people demonstrating certain aspects of dispositional diversity (Milton, 2014) are placed (Nadesan, 2005, Waltz, 2013). For example, a way of considering autism has been suggested that understands it as a serial collective, or a group of people so collected together because they share a certain relationship to the social and political world (Chapman, 2019). People are not characterised as autistic (either by themselves or by others) because they share certain essential features, but because they recognise in themselves (or others recognise in them) similar relations to disabling features in the world (Chapman, 2019).

Acceptance of the neurodiversity paradigm and the birth of critical autism studies embodies a change in the conceptualisation of autism and autistic people since its initial descriptions. This development accompanies changes in the way people as social agents are conceptualised more generally in the social sciences. The next section will discuss ethnomethodology and conversation analysis as examples of these alternative approaches to human sociality that are particularly fitted to the study of a neurodiverse social world.

\section{Ethnomethodology and Respecifying the Social}

Experiments are the dominant approach by which autistic people's social cognition or social abilities are studied in psychological research. In fact, psychology has largely moved the study of social behaviour into the lab and transformed sociality into performance on decontextualised tasks (Hollin, 2014; Rozin, 2001; Baumeister, Vohs, \& Funder, 2007). If we are to take seriously Nick Walker's (2013) proposition to dismantle the edifice of pathologizing psychological research, an alternative understanding of how to approach social phenomena is needed. Ethnomethodology is one such alternative.

Ethnomethodology is a policy or program of research originally developed by sociologist Harold Garfinkel (1967). Ethnomethodology concerns the study of people's everyday sensemaking procedures (ethnomethods, methods, or practices). Sensemaking does not refer to neuro-cognitive interpretations of the social environment around them, but instead refers to how people make the social environment sensible (i.e., intelligible). Ethnomethodology is concerned with both how participants make sense of social actions (Francis, \& Hester, 2004) as well as how participants produce their actions in ways that are intelligible to others (Heath et al., 2010). 
Garfinkel challenged the notion that social facts around identity (e.g., autism in the present case) or institutions (i.e. psychology as an academic discipline) were pre-existing objects to be understood as predictive or causal variables exerting some influence on what people do (Garfinkel, 2002). Respecifying social facts this way means that all social facts are concretely real but only in that they were produced that way. The apparent order of all social life, that social life happens at all, is a practical accomplishment.

The recasting of social facts as accomplishments also extends to scientific facts. Ethnomethodological research into the work of scientists has demonstrated how science is largely a constructive endeavour. Lynch (1985), drawing on ethnomethodology, described how scientific facts were the result of practices which produced docile objects, or rendered the desired aspects of the thing under study visible. A clear example is the staining of brain tissue in order to make individual component cells or structures individualizable. This notion of the docile object has been extended into the social and psychological sciences. Rose (1988) describes people as "ephemeral, they are shifting, they change before one's eyes and hard to perceive in any stable manner" (p. 188), and as such psychology developed "techniques for the disciplining of human difference" (p. 187). These techniques are both ideological and practical such that they afford the collection of details about people which render their psychological features stable and amenable to scrutiny while also producing a sense of the normal human variation against which people can be compared (Rose, 1988). In this way, psychological facts derived by scientific procedures are not merely reflections of a true empirical object, but are a new object in themselves, the result of interactions between phenomena and techniques.

Garfinkel's formulation of the accomplishment of social facts and order already provides an important re-casting of autism. Rather than thinking of autism, and by extension autistic people, as pre-existing entities in the world with features and characteristics to be understood by scientific investigation. Instead, the notions of autism and of autistic people are conceived as productions of social action. Autism does not refer to a thing that pre-exists and governs the cognition and behaviour of autistic people, it is a construct which groups together the attributed characteristics of a person (or group of people) and draws them together as a whole for various social, or scientific, means.

One thing that ethnomethodology concerns itself with is investigating the basis on which characteristics are drawn together to produce sensible categories like that of autistic 
people. One idea that is specifically relevant to the production and recognition of the category of autistic people is that of Trust. Spelled here with a capital to distinguish it from its mundane use, Trust refers to a particular attitude that members of a society must engage with regard to others' social behaviour without which social interaction cannot progress. Attitude here does not refer to a cognitive entity, but more of a way of approaching interaction. This attitude was formulated by Garfinkel (1963) in terms of three conditions. First, participants must be oriented to the same expectancies (i.e., playing by the same rules) as each other. Second, a participant must presume that their co-participants are oriented to the same expectancies as they are. Third, co-participants must also presume that of the first participant. When these conditions are not met, interactions would become troubled. Garfinkel demonstrated the trouble that could result from the breaching of the Trust conditions in his classic breaching experiments, showing that when this happens people become anxious in their search for something that makes sense of the other's currently non-sensical behaviour (Garfinkel, 1967).

The negative consequences of breaching Trust are not necessarily equal. The trouble that can result when Trust is breached has been shown to predominantly impact minority communities, such as racial/ethnic, gender/sexuality, or disabled minorities (such as autistic people) as the marginalisation they experience is the result of parties not being able to Trust that the same interactional methods are equally available (Turowetz, \& Rawls, 2019). When members of a society can generally Trust each other and breaches of Trust occur, certain kinds of breaches can be causally attributed by participants to one member, rather than to the mutual contribution of sense making methods to an interaction. Ethnomethodologists have discussed how this attribution occurs in the form of diagnosis of mental illness (Coulter, 1979). In this way, diagnosis of mental illness is a way of bringing someone's otherwise incomprehensible conduct into some kind of sense and makes available certain ways of managing troubles resulting from the breach in Trust (i.e., deployment of medical treatments, dismissal of conduct as symptomatic, etc cetera).

There is a clear link between ethnomethodology and critical autism and neurodiversity studies. Where ethnomethodologists are concerned with Trust, neurodiversity scholars and activists have formulated the same problem in the terms of the double empathy problem discussed earlier. Both approaches highlight the mutual responsibility for comprehensibility in social interaction. The double empathy problem is a specific case of the notion of Trust which highlights how autistic people are produced on the basis of, and as 
responsible for, difficulties in interactional intelligibility. In other words, people are rendered autistic on the basis of particular ways that Trust is breached in interactions with them.

How people are rendered autistic involves another key ethnomethodological idea; accountability. Trust requires that people presume they are using the same interactional methods the same way. Accountability refers to the sense made of an interactional participant's conduct, or the comprehensibility of one's action. Garfinkel wrote that social actors make their activity "visibly-rational-and-reportable-for-all-practical-purposes" (1967, p. vii) meaning that action is designed so that it is understandable for what it is or can at least be explained when required (ten Have, 2004). When sense cannot be made (like in a breach of Trust) some reparation attempts may be made (Garfinkel, 1967); people may be held to provide an account of their behaviour that makes it sensical. Alternatively, when it is clear there has been a breach in Trust and an interactional participant has demonstrated a misunderstanding of one's conduct, they may attempt to repair the breach themselves. For example, behaviours attributed to autistic people like echolalia (repetitions in speaking) or self-stimulatory behaviours (e.g., hand flapping) are commonly treated by neurotypical people as symptomatic of their autism. This treatment demonstrates the sense made of these behaviours by interactional participants; either the individual labelled autistic may be expected to make some sense of their behaviour, or some sense is made of it by attributing it to autism/madness/mental illness. Accountability will be discussed further in chapter four, where some of the ways the autistic children who participated in my research make behaviour sensible to other people will be analysed.

Tied to accountability is the notion of reflexivity, which refers to how participants actions are built both to show their understandings of previous action as well as providing their response (ten Have, 2004). Garfinkel called this display of understanding embodied in responsive action the documentary method of interpretation as the action itself is said to document or stand in for the interpretation of the previous action by doing what it does (Garfinkel, 1967, pp. 77-79). A demonstration of this is when a child is engaged in some activity it is not supposed to be (e.g., sneaking treats from the pantry) and after hearing their name called, they turn and lock eyes with their parent. At this point the child says "I'm hungry". This utterance demonstrates their understanding of what is happening as their parent catching them in the act and also provides a response to having been caught, by providing a justification for doing it. Autistic people are generally thought to make nonsensical contributions to conversations (e.g., echolalic repetitions are often considered meaningless; 
Korkiakangas, Rae, \& Dickerson, 2012), however considering their utterances in terms of the documentary method of interpretation would lead analysts to treat them as far from nonsensical, but actually produced in the service of making sense.

\section{Conversation Analysis and Studying Talk-in-Interaction}

Ethnomethodological ideas formed the backbone of conversation analysis, a method for studying naturally occurring social interaction initially developed by Harvey Sacks. Drawing inspiration from Garfinkel, Sacks was also concerned with the produced order of society and social action. He argued that this order does not just exist at aggregate levels requiring accumulated data and statistics to uncover" (Sacks, 1995), instead there is "order at all points" to be seen on a moment to moment basis, at both "higher" and "lower" levels in society (Schegloff, in Sacks, 1995). Conversation analysis adopts Sacks" "order at all points" conception of organisation and studies a particular locus of order, social interaction, as talk in social interaction is considered a "primordial site of human sociality" (Schegloff, 1987, p. 101).

Conversation analysis is a qualitative method through which we can study the ethnomethods used by members of a society to produce its order. Conversation analysts study the production and concretisation of the social facts Garfinkel was concerned with but specifically as that happens within naturally occurring talk-in-interaction (Sidnell, 2013). In order to do so, conversation analysts analyse social interaction with an ethnomethodological attitude in order to produce an emic account of how social interaction unfolds through the sequential ordering of practices designed to accomplish action.

My use of the term "design" above is not to imply that participants to social interaction are necessarily conscious of the way they deploy these practices or accomplish certain actions as much of the way talk is organised is done so outside of participants reportable conscious awareness (Sidnell, 2013). Instead, design here refers to how practices are treated by participants. Participants display an understanding, in the moment, that talk is engaged for a purpose in order to say, do, or mean something at that time and place. Conversation analysts depend on the naturalistic study of these in-the-moment displayed understandings of, or participant's orientations to, what is happening in any given interaction

\footnotetext{
${ }^{5}$ In fact, Sacks described how often poor statistical analyses seem to produce something ordered so that belief in aggregate order had to be challenged (1995).
} 
to describe what actions are being recognisably accomplished and the ways by which this happens (Goodwin, \& Heritage, 1990).

Talk is treated by conversation analysts (and by participants to said talk) as primarily a vehicle for action (Hoey, \& Kendrick, 2017), as a way to accomplish the everyday actions that constitute our social world (Schegloff, 1995). Actions are described by Schegloff (1996) as those accomplishments which compose the "moment-to-moment flow of daily life" ( $p$. 164). Such action includes greetings, invitations, offers, requests, as well as more complicated doings like marrying, sentencing, announcing as news and all other things that people do at every moment of their lives. These actions are said to be accomplished by practices, or verbal or non-verbal behaviours that have a unique character, that occur within certain local positions in the sequential order of talk and have particular implications for how the action it accomplishes should be understood (Heritage, 2011).

Ethnomethodological and conversation analytic work has been crucial in developing sociological critiques and alternative analyses of many areas of social inquiry and social life. One of the major domains (and most relevant for the issues of this thesis) this impact has been seen in is in the field of childhood studies where children's culture and participation in society is centred. This body of work will be briefly reviewed below as a demonstration of the kind of critical contributions ethnomethodology and conversation analysis can make to studies of social populations and practices.

\section{Children and Interaction}

For a long time, research on children and their social abilities took the position that children are developmentally deficient compared to adults and socialisation and development represents progress towards being fully complete adult members (Keel, 2016). Sacks (1995), however, spoke of a children's culture, referring to the set of cultural resources available to children for children's concerns. One interactional practice that Sacks (1995) refers to is the use of children's starters in the form of "you know what?" or similar and how this way of beginning a conversation managed the children's reduced rights to interactional access by making relevant the adult calling upon the child to proceed.

Treating children as if they have their own competences and culture is to treat them as members. A member, according to Garfinkel and Sacks (1970), is someone who displays an appropriate mastery of natural language. In other words, membership "refers to capacities or competencies that people have as members of society; capacities to speak, to know, to 
understand, to act in ways that are sensible in that society and in the situations in which they find themselves" (ten Have, 2002, para. 17). Research into children qua children, as Sacks exhorts us to do is to study the things children do as children to manage children's practical problems in the world. This attitude towards children's competence forms the basis of new social studies of childhood (James, Jenks, \& Prout, 1998). This approach to studying children as members of their own culture has been employed by conversation analysts (Butler, 2008; Kyratzis, \& Goodwin, 2017; Church, 2009; Stivers, Sidnell, \& Bergen, 2018).

Studies of children's talk have demonstrated their interactional competences. By looking at what children do, conversation analysts have shown children demonstrating sensitivity to the interactional contingencies operating upon themselves and their interactional partners. Through sophisticated use of interactional technologies children can attend to concerns around requests being granted (Wootton, 1981; Wootton, 2007), to interactional norms about who speaks when (Wootton, 2006), to power relations when told to do something (Kent, 2012), and to the use of category memberships to organise the activity of themselves and their peers (Butler, 2008). Looking at the interactional practices used by children, researchers can study children as children, rather than as adults-in-training (Mackay, 1991).

As well as looking at children's practices, ethnomethodologists and conversation analysists have studied the practices of parents and adults in their interactions with children. These studies show, for example, how adults claim or negotiate their authority over children (Cekaite, 2010), manage their children's attention (Cekaite, 2016), manage children's distress both verbally and physically (Kupetz, 2019; Cekaite, \& Kvist Holm, 2017), and scaffold children into preferred behaviours and understandings of those behaviours (Sterponi, 2009). Studies like these and of children's practices demonstrate how being a child and being a parent are mutually constituted in interaction with each other (Pontecorvo, Fasulo, \& Sterponi, 2001), and are not necessarily always predetermined, omnirelevant categories.

Ethnomethodological and conversation analytic studies of children's and parents' practices can be a model for how autistic people's interactional practices can be studied, as social agents with practices in their own right. But while the differences between children and adults are more inferentially available on the basis of visual observation (e.g., children are observably smaller), the differences between autistic and non-autistic people are typically 
primarily conceived as psychological. Discursive psychology provides an approach to analysing the interactional accomplishment of these psychological aspects.

\section{Accomplishing the Psychological: Discursive Psychology}

Discursive psychology is an approach to studying psychological topics by respecifying them (à la Garfinkel and ethnomethodology) into discursive or interactional achievements. Discursive psychology was originally developed to challenge some of the foundational assumptions in cognitive psychology about the relationships between the mind, behaviour and environment. Potter, Edwards, and Wetherell (1993) characterised the cognitivist worldview as being concerned with a threefold distinction of identity, mind, and reality, where reality was considered the unproblematic, obvious environment within which the other two objects were situated. While the environment is treated as transparently available to the researchers, the identity and the mind are treated as interdependent and less obvious, thus requiring investigation. Examples of this abound in cognitive psychological work, where researchers construct scenarios where there are correct and incorrect characterisations of or responses to the environment in the form of stimuli presented to participants that participants' behaviour can be compared against. For example, in the Mind in the Eyes task (Baron-Cohen, Jolliffe, Mortimore, \& Robertson, 1997), participants are asked to identify the emotional state of a person on the basis of just an image of the eye region of the face. The emotions in these images are predetermined such that there are correct and incorrect responses available to the participant. This is compared to emotional recognition, or attribution, in naturally occurring social interaction which is not a case of accurate identification but a mutual accomplishment in which attributions are made and accepted, rejected, or negotiated.

Discursive psychology challenges this characterisation of psychology and the environment. To Potter, Edwards, and Wetherell (1993), psychology cannot reasonably dismiss the environment as directly accessible and obvious, and adequate accounts of human psychology require consideration of the environment. This consideration of the environment cannot be straightforward, however, as any description of the world is, by definition, limited and selective (i.e., made on the basis of certain purposes for describing). Thus, psychologists cannot prioritise any description of the world and must instead incorporate an understanding of the constructions of different versions of the world in understanding human psychology (Potter, Edwards, \& Wetherell, 1993). 
This fundamental position within discursive psychology (i.e., that the world cannot be taken for granted and no description of the world has an a priori privileged status as a description) aligns with the critique of autism research made by critical autism and neurodiversity researchers. Descriptions of autism and autistic people have largely been given by scientists and doctors who treat them or study them through a lens of pathologisation. As such, these descriptions of the world and the autistic perspective of it have been privileged over other descriptions by autistic people themselves. A discursive psychological perspective, like the one taken in this thesis, disavows descriptions of the world from the analyst's perspective, instead focusing on the descriptions of affairs produced by autistic people themselves (and their interactional co-participants) and that are treated as relevant for the local ongoing action.

An implication of the acceptance that the world is not as transparent as it may seem is that this concern with constructed versions of reality extends to the other two objects of interest to psychologists, the identity and the mind, as they are themselves also components of the environment, reflexively constitutive of and constituted by the world they are situated within. As such, discursive psychology recognises that descriptions of the traditionally psychological world are as much versions as anything else. The typical divisions of the psychological, the perceptual, the cognitive, attention, memory, vision, language are descriptions that are also not to be taken as objectively concrete, but instead as divisions of human conduct described and utilised for a purpose. The task for the psychologist, having recognised this, is to understand how and why those versions are constructed.

Discursive psychology studies how versions of the world and the psychological are constructed and deployed through discourse for rhetorical purposes (Potter, \& Wetherell, 1987; Edwards, \& Potter, 1992). Discursive psychology challenged characterisations of language that emphasised its function as purely to provide descriptions of states of affairs (Edwards, \& Potter, 1992). This latter view of language held that language was primarily about encoding some information and transmitting it to another person to be decoded. Within a cognitive psychological world view this meant that talk about knowledge, memories, feelings, thoughts, and other mentalised phenomena were also just descriptions of real affairs occurring within an individual's mind. One way that discursive psychology does this is via the study of the use of the psychological thesaurus, or the use of recognisably psychological terms, for the accomplishment of various actions (Edwards, and Potter, 2005). 
However, beyond studying the psychological thesaurus, discursive psychological research also investigates how psychologically relevant notions like memory, emotion, intersubjectivity, agency, intention et cetera are built up or made available through people's action (Edwards, \& Potter, 2005). An example of this is the psychological notion of Theory of Mind. Because Theory of Mind has a prominent position in theories about autism and social functioning, an extended discussion of it and its discursive psychological respecification will be given in the next subsections.

\section{The Problem of Other Minds}

One of the specific findings behind the claim that autistic people are socialcognitively egocentric (i.e., cannot or tend not to engage in perspective taking) is the finding of a deficit of, or delay in development of, Theory of Mind cognition (Baron-Cohen, Leslie, \& Frith, 1985). Having a Theory of Mind means that one imputes mental states onto oneself and others (Premack, \& Woodruff (1978). Furthermore, this imputation of mental states is considered theoretical in a similar sense to theories in science due to the fact that mental states are (ostensibly) not observable and must be inferred from conduct, and they are used to predict further conduct (Premack, \& Woodruff, 1978). It is claimed that in autistic development the cognitive abilities that a Theory of Mind would depend upon do not mature, or mature late, limiting autistic children's ability to adequately infer others' mental contents and therefore understand and predict social behaviour (Baron-Cohen, 1991; Lombardo \& Baron-Cohen, 2010; Kimhi, 2014).

The initial evidence for an explanation of autism based on a Theory of Mind deficit came from assessment of false beliefs. Autistic children tended to rely on their own perspective when asked to report on the perspective of others (Baron-Cohen et al., 1985). Since this finding, many studies have claimed to show deficits in a range of Theory of Mind abilities, ranging from attributions of mental states to characters in a vignette (Happé, 1994), or to a person based only on an image of the eye region (Baron-Cohen, Jolliffe, Mortimore, \& Robertson, 1997), to more complex uses of beliefs to understand emotions (Baron-Cohen, 1991) and make moral judgements (Margoni, \& Surian, 2016).

The Theory of Mind deficit hypothesis has held sway over the way autism has been conceived since Baron-Cohen et al.'s (1985) paper (Gernsbacher, \& Yergeau, 2019). However, there have been serious challenges to the centrality of Theory of Mind deficits in autism. Theory of Mind deficits themselves have been proposed as the result of some other 
interactional capacity that is itself the cause of autism such that Theory of Mind deficits are common in, but not causal of, autism (Boucher, 2012), and Gernsbacher and Yergeau (2019) demonstrate in their review that Theory of Mind research on autistic people commonly fails to replicate and that the tasks developed to assess it exhibit poor convergent and predictive validity. These kinds of critiques have led some to suggest abandoning Theory of Mind not just in thinking about autism (Gallagher, 2004) but in thinking about social interaction as a whole (Gallagher, 2020; see also volume 14, issue 5 of Theory \& Psychology, 2004).

\section{Mind-Reading? Or, Social Practice?}

An alternative to the study of Theory of Mind is put forward by discursive psychologists (Antaki, 2004). Discursive psychologists take an anti-cognitive stance towards language and behaviour and focus their attention on the accomplishment of social action rather than cognition (Edwards, \& Potter, 1992). As discussed earlier, discursive psychology holds the view that social interaction proceeds not via inferences of, and prediction from, mental state theories, but instead through a situated and displayed analysis of, and response to, recognisable social practices that are resources to build intersubjectivity and progress coordinated actions (Edwards \& Potter, 1992; Edwards, 1997; Garfinkel, 1967; Schegloff, 1992). Rather than being situated in a private world of the mind and positioned as occurring prior to, and motivating, behaviour, mentalised constructs like knowledge, emotion, and memory are construed as publicly displayed in our social conduct.

Understanding language as the medium through which action is conducted (rather than as reference to, or description of, things like mental states) has substantial implications for the problem of understanding social action that the Theory of Mind account tries to solve. It addresses this problem by bracketing off the mind's cognitive processes as underpinning behaviour (or even denying its relevance altogether; Coulter, 2008) and studying only that which is public and visible. Where a Theory of Mind approach retains an interpretative gap between the observed (i.e., behaviour) and the thing theorised (i.e., intention, knowledge, emotions, et cetera), the discursive approach places the thing theorised very concretely in the observable world (Edwards, 2012). As such, things traditionally thought of as internal states become publicly available actions within an interactional, sequential space and participants produce the meaning and relevance of those public behaviours in their responses to them. Theory of Mind is respecified as the ability to appreciate and coordinate the social actions (and their implications) conducted by one's interlocutors (Antaki, 2004; Bottema-Beutel, 
2017). The primary problem being solved by interlocutors is not what is the intention behind a behaviour, but what is an appropriate next move (Enfield, \& Sidnell, 2017).

This discursive concern with the appreciation and coordination of public social actions has at least three, major, implications for research into autism. First, it positions the kinds of things that Theory of Mind was proposed to address as something that can be empirically studied in naturally occurring interaction. This is in contrast to the experimental approach typically taken to study Theory of Mind. Experimental assessments like this suffer from low convergent (Warnell, \& Redcay, 2019) and ecological validity (e.g., tests of Theory of Mind do not correlate with more naturalistic assessments, proposed to be due to cognitive compensation; Livingston, Colvert, the Social Relationships Study team, Bolton, \& Happé, 2019). Secondly, Theory of Mind assessments are social interactions in their own right. As a result, the way the testing scenario plays out as an interaction can impact how participants provide their responses to the task and how those responses are understood as competent, appropriate responses (Maynard \& Turowetz, 2017; Korkiakangas, Dindar, Laitila, \& Kärnä, 2016). Third, it allows researchers to document the collaborative work that goes into understanding our interlocutors. The discursive approach reformulates understanding our interlocutors into a two-person problem; Namely, each participant engages in ensuring that one's understanding of what another is doing/thinking/feeling/ et cetera is jointly accomplished, rather than an individualised accomplishment of separate, theorising brains. This is specifically important in the context of autism as the difficulties that people have in interaction when a participant is autistic are usually attributed to the autistic participant only and represented as being the result of their individual cognitive deficits. However, it has been pointed out that when these kinds of interactional difficulties occur, they are often as much due to the non-autistic participant not understanding the autistic one as the other way around (Heasman \& Gillespie, 2018). Milton, Heasman, and Sheppard (2018) refer to this as the double empathy problem, highlighting the idea that while autistic people may have difficulty interacting with neurotypical people, neurotypical people also experience difficulty interacting with autistic people.

Previous conversation analytic work has both directly and indirectly commented on the issues surrounding Theory of Mind. Harvey Sacks admonished that analysts should not be concerned with how or whether people are thinking when they act, instead they should just focus on their actions (Sacks, 1992). Schegloff (1992) discussed how a turn at talk demonstrates understandings of the turn preceding it. For example, when someone launches a 
request for some piece of information and another provides that information then the provider has demonstrated their understanding of that turn as a request for information.

Discursive psychological work has also looked at the social behaviours presumed to require Theory of Mind by looking at cases of talk by people with schizophrenia. These studies have shown people with schizophrenia demonstrating their understandings of, and concerns with, others' mental states through direct reference to mental states, like emotions and beliefs, and also how they project their interlocutor's turns (McCabe, Leudar, \& Antaki, 2004). As well, Antaki (2004) demonstrated how turns at talk from schizophrenic patients which may appear to demonstrate Theory of Mind deficiency can be understood as displaying an understanding of the local interactional contingencies and thus these speakers are highly competent rather than deficient. Another study of Theory of Mind in autism has been conducted using conversation analysis, but not from within discursive psychology (KremerSadlik, 2004). This study argued that autistic children's answering of parents' questions was not just a case of recognising the questioner's intentions but required that the children understand socio-cultural norms around actions and the conditional relevance of first pair parts.

An implication of discursive psychology's concern with the naturally occasioned accomplishment of the psychological in social interaction is that discursive psychology removes psychological research out of the laboratory and into the mundane settings where social interaction happens (Huma, Alexander, Stokoe, \& Tileagă, 2020). Psychological work is part of the social world outside of the laboratory, and so researchers need to study what happens in those mundane settings in order to grasp how that psychological work is done and what it is done for. A practical issue for the discursive psychologist is to find those settings where the psychological phenomenon of interest occurs in order to collect data on its occurrence. To do this, discursive psychology draws inspiration from ethnomethodology in the form of the perspicuous setting. Perspicuous settings are those interactional contexts where the phenomenon of interest is particularly relevant to the action that goes on in that context and is thus most clearly observable (Vom Lehn, 2014). For example, consider the study of physical affection by autistic people. A perspicuous setting for such a study would be one in which there would be autistic people and (ostensibly) opportunities for physical affection. In the case of this thesis, the family home was chosen as a perspicuous setting for the study of autistic children's everyday activities, including physical affection. 


\section{Discursive Psychology for Critical Autism Studies}

The previous sections of this chapter outlined ethnomethodology, conversation analysis, and discursive psychology as approaches to conceptualising and studying the social world, and specifically the production and management of the psychological in social interaction. Links between these and some of the perspectives within the neurodiversity movement and critical autism studies were also made, for example in discussing the double empathy problem and Garfinkel's Trust argument.

Above, I described Dinishak's (2016) critique of the deficit approach to studying autism; that it was not just ethically, but scientifically disadvantageous to take a deficit approach as it tended to leave autism with only a negative picture of what constitutes it and ignores qualitative differences that may be missed (as deficits are largely a quantitative issue). Discursive psychology is a way to study autism and autistic people's behaviour that avoids these issues by making positive observations, focusing on competency and mutual accomplishment. Because discursive psychology analyses what actually occurs in social interaction, observations made about what autistic people do can only be based on what is actually recorded. Rather than setting an arbitrary task and seeing whether a person can accomplish it or not, discursive psychology focuses on what kinds of things autistic people might freely get involved in and how they accomplish whatever tasks their day to day lives and the people, objects, and events in them - would otherwise naturally require of them.

Discursive psychology can also be strongly aligned with the critical, emancipatory aims of critical autism studies ${ }^{6}$. Critical autism researchers are concerned with providing "new, enabling narratives of autism" (Davidson, \& Orsini, p. 12) and critiquing the pathologising, individualising approaches common in studies of autism (Woods, Milton, Arnold, \& Graby, 2018). Discursive psychology is strongly concerned with critiquing narratives in psychology that remove not just the person but the environment as well (Potter,

\footnotetext{
${ }^{6}$ Ethnomethodological, conversation analytic and discursive psychological research have slightly different approaches to concerns around political aims like emancipation. One of ethnomethodology's historically core ideas is the notion of ethnomethodological indifference. The policy of ethnomethodological indifference was described by Garfinkel and Sacks (1970) as "seeking to describe members' accounts of formal structures wherever and by whomever they are done, while abstaining from all judgements of their adequacy, value, importance, necessity, practicality, success, or consequentiality." (p. 345). While this notion is central, some discursive psychological and other conversation analytic research re-evaluated to what extent analysts should be indifferent and towards what we are indifferent. Studies of prejudice (e.g., Whitehead, \& Stokoe, 2015) and of sexuality and gender (Speer, 2002) have relied on theory and made judgements of the nature of some talk (e.g., as racist or sexist) in order to select targets for analysis. I argue that a sequential analysis of talk as it occurs requires a certain degree of indifference to understand participants' orientations, however, once that analysis has been done, the understanding of the structure of that analysed interaction is now available for theorising.
} 
Edwards, \& Wetherell, 1993). In this way, discursive psychology is engaged with the general concern, of which the critical autism perspective is a more specific case.

There is also now a strong encouragement for autism researchers to increase focus on research that "acknowledge[s] the need to address the everyday realities of autism" (Pellicano et al., 2018, p. 81) as well as increasing the participation of autistic people in the research to add an autistic voice (Chown et al., 2017; Milton, \& Bracher, 2013). Discursive psychology does this by only using data would pass the unwell social scientist test (Potter, \& Shaw, 2018), or data recordings of events that would have happened whether the researcher was studying it or not. By studying autistic people in their naturally occurring activities, discursive psychology provides accounts of how these things happen and what the autistic participants showed was doable or difficult. As well, discursive psychology (from its ethnomethodological and conversation analytic roots) is concerned with producing an emic account of social interaction. This means that, while an analyst is the one writing the account, they can only write what the participants clearly demonstrate is important for understanding the interaction as it happened. In this way, priority is given to the interpretations of the autistic people and their interaction co-participants over the analyst's. This has been argued in other contexts where the participants to research or the activities they engage in are less amenable to study in other formats, for example, young children (Bateman, 2017).

\section{Summary}

This chapter has introduced the theoretical approaches taken in this thesis. While the neurodiversity paradigm provides a metatheory for recasting autism as psychological difference, ethnomethodology was introduced as a way of recasting the social from one of pre-existing identities with mentalised intentions to one of actions and normative accountability. Discursive psychology, as an approach to psychology informed by ethnomethodology and the method of conversation analysis ethnomethodology inspired, has also been described.

Ethnomethodology, conversation analysis, and discursive psychology have been demonstrated to align with the principles of the neurodiversity paradigm and with the goals and aims of critical autism studies. This has been done by demonstrating ethnomethodology's general approach to Trust in social interaction and the production of social facts (the general theorisation of the double empathy problem), the study of autistic people's real lives in interactional settings that naturally impact them (versus contrived laboratory tasks), and a 
demonstration of how ethnomethodology and discursive psychology have produced critical accounts of the social lives of children/parents and Theory of Mind and social cognition, respectively. The next chapter will conduct a review of the literature on autism, language, and social interaction. 


\section{Chapter Two: \\ Autism, Language and Social interaction}

"The outstanding, "pathognomonic", fundamental disorder is the children's inability to relate themselves in the ordinary way to people and situations from the beginning of life."

(Kanner, 1943, p. 242).

Descriptions of autism have always focused on the social behaviour of autistic people as fundamental to the diagnosis. For instance, Kanner (1943) noted not just tendencies for autistic children to avoid or ignore other people but also behavioural and linguistic differences when they did engage in social interaction. Increasingly, language became a prime focus in autism research with all domains of linguistic ability investigated for possible delays or apparent deficits. Ultimately, while a variety of patterns in language ability across linguistic domains were found (e.g., phonology, morphology, syntax; Tager-Flusberg, 2009), pragmatics, or the use of language in social contexts, became a central focus (Wilkinson, 1998; Loveland, Landry, Hughes, Hall, \& McEvoy, 1988).

In this chapter, I introduce some of the research literature on autism, language and social interaction. Firstly, some of the core cognitive theories that have held sway in explaining autism will be introduced and discussed with regard to how they claim to explain the social behaviour of autistic people. Then, research on language and social interaction and autism will follow, beginning with findings from psycholinguistics and aligned fields and conversation analytic research. Lastly, research from the more recent trend of investigating social touch in autism will be described as a movement to reposition the social concerns in autism away from just verbal language differences towards including autistic embodiment in interaction.

\section{Theorising Autism in Psychology}

Since autism's beginnings as a psychiatric diagnosis, ostensive social and linguistic deficits have been considered central to it. Leo Kanner first described infantile autism in his 1943 paper, “Autistic Disturbances of Affective Contact”. Kanner's assessment of 11 children brought to his clinic lead him to describe a syndrome with the core deficit being "the children's inability to relate themselves in the ordinary way to people and situations from the 
beginning of life" (Kanner, 1943, p. 242). This core deficit manifested in a selection of common traits, including; an "extreme autistic aloneness" or disregard/disinterest in the external world and other people, certain difficulties with language (i.e., being mute, speaking only to name objects or repeat things previously said, misuse of first- and second-person pronouns), extreme rote memory, anxious attempts at the "maintenance of sameness", and limited behavioural repertoire and/or interests (Kanner, 1943). The year after Kanner's description was published, Hans Asperger (1944, translated by Frith, 1991) described a syndrome similar to Kanner's infantile autism which he called autistic psychopathy.

Although the emphasis on social and relational difficulties has remained in descriptions of autism, different theories have been proposed to explain why these difficulties arise. In the 1980s, cognitive explanations of autism became popular. These kinds of theories position the behavioural features of autism as the result of disordered higher-level, abstract, cognitive processes. These processes are not necessarily obviously linked to social ability but have widespread impact due to their basic nature. Amongst a large diversity of theories now proposed, three specific theories have dominated the intersecting space of autism, cognitive psychology, and cognitive neuroscience (Hollin, 2014). These theories concern deficits in executive functioning, meta-representation and Theory of Mind, and what is called central coherence.

As discussed in Chapter One, social interaction has been thought by many to require a Theory of Mind in order understand and respond to behaviour. The Theory of Mind deficit hypothesis has been central to autism theorising since it was proposed in a study by BaronCohen, Leslie, and Frith (1985). Three key ideas came together in the production of this theory (Hollin, 2014). The first was Premack and Woodruff's (1978) article discussing the notion of Theory of Mind. According to them, humans impute mental states to themselves and to others and it is by this imputation that we understand our own and others' action and intention. This capacity to conceive of others' minds is considered central to human social ability. The second was the development of a theory of meta-representations. Leslie (1987) wrote that children must develop a capacity to build cognitive representations that are not susceptible to change based on pretence. For example, your notion of what a cup is should not change based on you having pretended that a cup was a hat once. In order to retain these stable notions despite being able to conceive or and engage with a variety of objects in a variety of ways and contexts that may or may not involve fantasy or pretence, there must be higher order stable representations that are kept. The third was the observation by Wing, 
Gould, Yeats, and Brierly (1977) that autistic children did not tend to engage in pretend play. In their study, they found that eight out of twelve autistic children did not engage in symbolic play (i.e., play that involved some kind of pretending, for example pushing a toy and pretending to mow lawns with it) and the remaining autistic children engaged in stereotyped play, and as a result they claimed that the absence of symbolic play is closely linked to autism (Wing et al., 1977; however, later work suggests that autistic children engage differently in both symbolic and functional play; Jarrold, Boucher, \& Smith, 1993).

Baron-Cohen, Leslie, and Frith (1985) proposed that autistic children did not possess a Theory of Mind on the basis that in order to understand oneself and another's cognition, you would need to have a meta-representation of cognition itself to preserve notions of your cognition and other's cognition that would otherwise impact each other. They found support for this claim in the form of the Sally-Anne test which required a participant to be able to conceive of what someone else might be thinking in order to pass it. Most autistic children failed this test, and those that did pass it failed a second-order version of the test (which required being able to conceive of what someone thought about someone else; Baron-Cohen, 1989). The Theory of Mind deficit hypothesis has been a cornerstone of autism theorising, with people adopting the terms mindreading and mindblindness to refer to the different social abilities of typically developing people and autistic people (Baron-Cohen, 1997).

Executive functioning deficits have also been proposed as a fundamental contributor to autism. Executive functions are generally said to include working memory, planning, attention and response inhibition (Chown, \& Beardon, 2017). Executive dysfunction is associated with social impairment (Leung, Vogan, Powell, Anagnostou, \& Taylor, 2016; Torske, Nærland, Øie, Stenberg, \& Andreassen, 2018) and autistic people are thought to be impaired in some or all subdomains of executive functioning (Pennington, \& Ozonoff, 1996; Hill, 2004). For example, Ozonoff and Jenson (1999) found that autistic participants scored much worse than typical control participants in a Tower of Hanoi task (a task which requires participants to plan out steps of moves to arrange a set of items in a stack). However, evidence has been varied and inconsistent. For example, Pennington and Ozonoff (1996) show that autistic people have a pattern of executive function deficits that distinguish them from those with attention-deficit/hyperactivity disorder. However, a meta-analysis by Demetriou et al. (2017) of all studies of autistic people's executive function led them to conclude that autistic people did generally have impaired executive function and that this impairment was not domain-specific, suggesting the autistic executive dysfunction profile 
could include all executive function domains. Although executive function deficits seem to be implicated in social behaviour, the evidence is mixed concerning the link with autistic people's social abilities.

Weak central coherence is another major factor presumed central to autism. The weak central coherence hypothesis (Frith, \& Happé, 1994; Happé, \& Frith, 2006) suggests that what distinguishes autistic from neurotypical individuals is the differential tendency to appreciate the gist, or gestalt wholes, over the details that comprise them. While neurotypical people tend to orient to 'the bigger picture', autistic people are suggested to prefer to concentrate on details and component parts. For example, autistic participants have been found less likely to succumb to visual illusions compared to neurotypical participants and this has been explained as a result of tending not to integrate the details of the image into a perceptual whole (Happé, 1996). There is only minimal evidence that weak central coherence is associated with the sociable abilities of autistic people (Russel-Smith, Mayberry, Bayliss, $\&$ Sng, 2012), but this theory aimed to explain thing other theories tended to ignore, suggesting that weak central coherence as a perceptual bias might occur alongside differences in social cognition (Happé, \& Frith, 2006).

It has been argued that these abstract cognitive capacities contribute to social behaviour via several pathways. For example, it has been suggested that domain-general cognition (e.g., aspects of executive function and central coherence processing) support Theory of Mind in domain-specific cognition (Pellicano, 2010). Ultimately, academic consensus is that, while these things are commonly implicated in autism, there is no explanation of autism based on singular cognitive processes (Pellicano, 2010; BottemaBeutel, Kim, \& Crowley, 2019).

Many other theories have been put forward to explain autism besides the ones just described (Chown, \& Beardon, 2017). Cognitive explanations all share a basic property; they focus attention on the contributions of individual differences to someone's appearing autistic. This individualised, cognitive focus, along with the development of neuroscientific technologies, has afforded the search for explanations the capacity to investigate a wide variety of possible individual contributions to one's appearance as autistic, including searching further inwards into the individual to find neural and genetic substrates that underly the proposed cognitive deficiencies. For example, neuroscientific work has shown "anatomic abnormalities" (Baron-Cohen, 2004, p. 945) in the structure and function of multiple different 
brain regions, and many studies have investigated genes possibly implicated in autism (Geschwind, 2011). Research into what individuals bring genetically, neurologically, and psychologically, to their environmental circumstances (e.g., to social interactions) is important as these constitute some of the resources available to the individuals in managing and responding to those circumstances. However, it will be seen from the above descriptions of cognitive explanations of autism, that they focus on how more general or basic cognitive capacities (e.g., reasoning about beliefs, holistic versus detail-oriented perception, and executive function) contribute to autism. While these theories focus attention on the general cognitive capacities of individuals, attention can also be directed towards an understanding of specifically social cognition and behaviour. The next section will discuss the research that looks specifically at autistic individual's abilities in specifically social domains.

\section{Studies of Atypical Interaction and Autism}

The study of autistic people's social interaction tends to come under the domain of atypical interaction. Atypical interaction is characterised by at least one participant to the interaction having a disability or impairment that consequentially impacts the talk they are party to (Wilkinson, 2019). Although social interaction commonly involves troubles in speaking, hearing or understanding, atypical interaction is typically considered distinct from these normal 'problems' on the basis that the troubles are linked to some impairment or disability, such as hearing impairments, speech impairments, or cognitive impairments (Wilkinson, 2019). While the study of these interactions has long been conducted within different academic and professional fields and subfields (i.e., psychology, speech and language pathology/therapy, clinical linguistics et cetera), in the last 30 years, naturalistic studies of these kinds of interactions utilising conversation analysis have become increasing popular (Wilkinson, 2019; Wilkinson, Rae, \& Rasmussen, 2020).

\section{Language and Autism}

Autistic children commonly develop language differently to neurotypical children. Studies have shown that a proportion of autistic individuals will never use language (Kim, Paul, Tager-Flusberg, \& Lord, 2014). The exact proportion of non-verbal autistic people has been debated to range between around 20 percent up to 50 percent (Kim et al., 2014; Anderson et al., 2007; Rutter, 1978). Kjelgaard and Tager-Flusberg (2001) found that about $25 \%$ of their sample of children diagnosed with autism could be categorised as having normal language skills while the rest displayed some degree of language abnormality. 
Clinical linguistic research tends to suggest that autistic people's speech delivery differs from neurotypicals, but much of this research has been inconsistent or inadequately studied (like in the context of prosody; Arciuli, 2014). Studies of articulation have claimed that autistic children tend to be delayed in their phonological development (Bartolucci, Pierce, Streiner, \& Eppel, 1976), and that differences in articulation can persist even when other language abilities are normal (Cleland, Gibbon, Peppé , O’Hare, \& Rutherford, 2010). Research into prosody has resulted in widely varied conclusions (McCann and Peppé, 2003), but it is consistently claimed that stress (particularly the use of contrastive stress; Paul, Augustyn, Klin, \& Volkmar, 2005) and recognition of the pragmatic uses of prosody differ (Diehl, Bennetto, Watson, Gunlogson, \& McDonough, 2008).

Pragmatics, or language use in context, is thought to be the central language domain affected by autism (Wilkinson, 1998). Kanner (1943) described elements of autistic children's talk as uncommunicative. Research has suggested that autistic children do not develop their discursive skills alongside their structural language abilities at the same rate as typically developing children (Tager-Flusberg, \& Anderson, 2001) and this pattern of development occurs even in children who achieve "optimal outcomes" in development after diagnosis (Kelley, Paul, Fein, \& Naigles, 2006).

Many pragmatic differences have been found in connection with autism. Autistic children tend to respond incorrectly, or not at all, to other people's attempt to coordinate their attention (Loveland, \& Landry 1986; Loveland, Landry, Hughes, Hall, \& McEvoy, 1988) and tend not to initiate joint attention with others (Shumway, \& Wetherby, 2009). When they do, research suggests they may have a restricted range of behaviours and use those behaviours incorrectly (e.g., primarily using questions to initiate interaction rather than as sincere requests for information unknown to the questioner; Hurtig, Ensrud, \& Tomblin, 1982). More specifically, children with autism display reduced use of comments that provide new topical information, affirmations, and turn taking vocalisations/continuers (Tager-Flusberg, \& Anderson, 1991; Loveland et al., 1988). Experimental evidence shows autistic children display reduced consideration of the Gricean maxims in judging the appropriateness of responses to questions (Surian, Baron-Cohen, \& Van der Lely, 1996). Although autistic children may have a restricted repertoire that they use, pragmatics research suggests they can comprehend more than they can use. For example, children with autism are just as able to comply with a request regardless of the grammatical form the request takes (i.e., imperatives 
versus interrogatives or declaratives; Kissine, De Brabanter, \& Laybaert, 2012; Kissine et al., 2015).

By focusing on language pragmatics, research into autism and language gets closer to studying the actual phenomenon of autism as it is manifest in the social world. A critique that can be held against this research, however, is that it paints a picture of autism and social language disorder that depends on external evaluation by analysts and the application of decontextualizing research methods like experiments. As such, the picture of autism and language disorder revealed is one that is heavily determined by the presumptions of researchers about what is normal or appropriate social language use, as all methodological choices embody presumptions regarding autism and what competence would look like; Dindar, Lindblom, \& Kärnä, 2017. In the next section, research into social interaction based in conversation analysis will be reviewed, showing how an approach to language and interaction that emphasises the participants' own judgements of appropriateness provides some nuance into discussions about autistic people's social interaction.

\section{Naturalistic and Conversation Analytic Studies of Autism}

An important feature of the above discussed research into autism and language and is that results are based on researchers' decontextualised, outside judgements about the suitability of participants' talk. Language is generally considered unproblematically isolable and transparent such that autistic people's use of language can be compared against some standard of appropriateness or correctness. In comparison, conversation analytic research into autistic social interaction focuses on how talk is accomplished between interactional parties and so the emphasis is not on correctness but on participants' orientations to local, interactional suitability or talk and the actions accomplished by it. This subsection will review the conversation analytic research into autistic social interaction.

Since the first conversation analytic study of autistic talk (Frankel, 1982) researchers of atypical talk have had a special interest in autism. One way that conversation analysis has been used to study autism is by looking at the technical procedures by which someone is diagnosed as autistic in the first place. By looking at how assessments of possibly autistic children are made (Maynard, \& Turowetz, 2017a; Korkiakangas, Dindar, Laitila, \& Kärnä, 2016) as well as how medical professionals use information from their assessments to construct a child as autistic (Maynard, \& Turowetz, 2017b), conversation analysts have 
shown the interactional mechanisms and practical reasoning involved in producing autism out of children's behaviour.

Beyond the diagnosis of autism, conversation analysts have studied features of autistic people's talk that are typically considered symptomatic presentations of autism. The most heavily studied of these has been echolalia, or the repetition of utterances (Stribling, Rae, \& Dickerson, 2007; Sterponi, \& Shankey, 2014; Local, \& Wootton, 1995; Sterponi, \& de Kirby, 2016), however other features like, perseverative topic talk (Stribling, Rae, \& Dickerson, 2009), atypical pronoun usage (Sterponi, \& de Kirby, 2016), "problematic" physical behaviour (Damico, \& Nelson, 2005), and social inflexibility (Muskett, Perkins, Clegg, \& Body, 2009) have been analysed as well. These kinds of studies look into the local, functional properties of the so-called symptomatic behaviours or utterances, commonly finding that these behaviours are actually not symptomatic or disordered but are, in fact, coordinated social actions, deployed by the children to attend to local interactional contingencies (Sterponi, \& de Kirby, 2016; Sterponi, de Kibry, \& Shankey, 2015). For example, certain echolalic responses seem to be used in order to increase the chances that the utterance is heard in contexts where the child orients to possible hearing difficulty by their recipient (Stribling, Rae, \& Dickerson, 2007), similarly what appears as inflexibility in interaction can actually constitute attempts to retain authority over the ongoing course of action (Musket et al., 2009) or solicitation of responses in environments of low up-take (Bottema-Beutel, Louick, \& White, 2015)

Conversation analytic research has looked at how autistic people manage interactional contexts in which they are thought to be impaired. Considering the impact Theory of Mind theories have had on understanding autism, some conversation analysts have examined moments in which joint attention and mutual understanding have been explicitly accomplished in interactions with autistic people. These studies have looked at the accomplishment of joint visual attention through pointing (Dindar, Korkiakangas, Laitila, \& Kärnä, 2016a) and through mutual gaze (Korkiakangas, \& Rae, 2014), the management of interaction troubles and repair (Dindar, Korkiakangas, Laitila, \& Kärnä, 2016b), and autistic children's awareness of, and response to, other people's assessment of their bodies (Ochs, 2015). Despite claims that mutual understanding and engagement are tough for autistic people, they can be capable of managing these complex joint-attentional issues in interaction. 
There is also a growing conversation analytic literature on autism in family life and in the home (see the ethnography of autism study by Ochs and colleagues; Ochs, \& Solomon, 2010). Using naturalistic data and interactional methods, specific interactional phenomena (e.g. how autistic children begin and contribute to narratives [Solomon, 2004], participate in moral discourse in a variety of ways [Sterponi, 2004], and are scaffolded in question-answer sequences [Kremer-Sadlik, 2004]) as well as broader ideas around autism and sociality have been investigated. Autism has been understood within language socialisation theory, arguing that the interactional challenges faced by autistic individuals is similar to those of secondlanguage learners. While noting there is some overlap between the communities of autistic and non-autistic people in terms of how certain actions are accomplished (like between language communities), there are also large differences which have to be managed on the go in the midst of social activity (Ochs, 2002). Situating autism and autistic people in this broader realm of sociality and culture highlights what is typically taken for granted about social interaction seen from a neurotypical perspective.

In arguing for this socio-cultural, situated approach to autism, a distinction between interpersonal and socio-cultural perspective taking was proposed to address issues around Theory of Mind and autism (Ochs, Kremer-Sadlik, Sirota, \& Solomon, 2004). In so doing, the interpersonal perspective-taking (i.e., concern with other's traits and mental states) commonly emphasised in Theory of Mind Conceptions could be put aside to examine how autistic individuals manage interaction in terms of socio-cultural perspective-taking (i.e., management of turn-taking, interactional sequences, indexicality and inference et cetera). Doing this made visible the cline of difficulty that autistic children tended to demonstrate, where turn-taking and local structure were easier to manage and indexicality and inferences with reference to cultural knowledge were harder (Ochs, et al., 2004). Despite this cline of difficulty, autistic children studied showed an awareness of the observability of their bodies (Ochs, 2015), and the linkages between their behaviour and inferable identities (Ochs, \& Solomon, 2005).

Ochs and Solomon (2005) proposed two kinds of non-exclusive explanations for autistic children's social competence. One was that their common interlocutors (e.g., family, friends, teachers) were generous in designing their interactional moves for the autistic participants in order to support them. This is supported by other studies showing how interactions in which autistic children participate are scaffolded by their interlocutors (Rendle-Short, 2002; Rendle-Short, 2014). The other was that the autistic children themselves 
had practices for managing interactional difficulties. Ochs and Solomon (2004) described two; make interactional contributions that were sequentially appropriate despite being possibly only-partially relevant topically; shifting discussion to topics with less personal, more objective cultural content. In this way, social competence is seen as the interactional result of input from both autistic people themselves and their interlocutors, rather than solely internal to the individual.

There is a tension in many of the conversation analytic and such aligned research when it comes to atypical talk. While many of the studies reviewed here are based on phenomena that have been characterised as disordered in the interactions of autistic people, the approach to these phenomena and findings regarding them tend to challenge the notion that they are the results of disordered cognition or are symptomatic and therefore nonfunctional. In fact, these studies tend to show that these phenomena serve important conversational functions and can be recognised as communicative by interlocutors. While it is the case that some of autistic people's conduct stands out to neurotypical people as odd (this must be the case, or else what is the basis for having them assessed and diagnosed?) that can be true while also accepting the fact that they are publicly displaying their practical, inthe-moment reasoning, making their working logic clear. So, while interaction with autistic individuals might appear atypical, that it is characterised that way demonstrates the breach of Trust whereby neurotypical interactants can no longer take for granted that their logic is immediately, mutually comprehensible.

\section{Methodological Fusion: Conversation Analysis and Experimental Psychology}

The previous two subsections have treated the experimental and conversation analytic research into social interaction and autism as quite distinct. This thesis specifically focuses on practices in social interaction and their description with conversation analysis, but it is not necessarily the case that conversation analysis and experimental studies are exclusive or opposed to each other. There is a push by some sympathetic to both conversation analytic and experimental research to combine the two approaches. de Ruiter and Albert (2017) argue for a methodological fusion, claiming that conversation analysis and experimental psychology have much to offer each other. Specifically, they argue that conversation analysis can aid in addressing psychology's replication crisis via it's careful explication of naturally-occurring phenomena, and that experimental approaches can aid conversation analysis by using the 
patterns found through conversation analysis to generate testable and falsifiable hypotheses (de Ruiter, \& Albert, 2017) ${ }^{7}$.

What might a fusion of conversation analysis and experimental psychology look like? Kendrick (2017) (in arguing against the false dichotomy between naturalistic and experimental methods) specifies some ways experimental methods may support conversation analysis. He suggests that experimental studies can help to refine and test conclusions drawn from conversation analytic empirical findings. An example of this is the study by Heritage et al. (2007). Based on previous conversation analytic work on the marked anticipation of polarity in questions (specifically, that use of the word any in questions like do you have any other medical problems? anticipates a negative response, while the word some anticipates a positive response), Heritage et al. (2007) assigned doctors to experimental conditions where they were to ask their patients Is there anything/something else you would like to address in the visit today?. They found that patients asked with something tended to present more issues than those asked with anything. This experimental study is thus argued to have strengthened the claim concerning the polarity of such items as some or any. This kind of approach has been utilised in other research domains, for example in cognitive neuroscience studies investigating the neurological underpinnings of practices as enacted in interaction (Bögels, \& Levinson, 2017).

In the approach demonstrated by the Heritage et al. (2007) example, conversation analytic conclusions are further tested and explored through experimental methods. A related approach is to use experimental psychological findings in order to constrain and validate the analyses we make of interactional data. Conversation analysis is generally analytically anticognitivist ${ }^{8}$. However, some have argued that experimental findings can be used to validate and support claims made from conversation analysis by reference to what might be cognitively or neurologically possible (Sanders, 2005). For example, findings from

\footnotetext{
${ }^{7}$ A similar argument is also made by Rendle-Short (2019). Specifically in the context of research on autistic social interaction, she wrote that researchers in both quantitative and qualitative spaces should be engaging with the both quantitative and qualitative findings, as one provides a view of the "big picture of what 'mostly' or "usually' occurs" (p. 276) in atypical interaction, while the other provides the details of what happens when it does. It is important, however, to consider how this kind of approach presumes that qualitative and quantitative research would be expected to provide converging findings and coherent conclusions which is not necessarily the case or the goal (Maracek, 2003).

${ }^{8}$ By "analytically anti-cognitive", I mean that conversation analysts (regardless of what they apply their findings to argue about), tend not to refer to cognition when explaining the structure or unfolding of any given interaction. In this, analysts are following Harvey Sack's (1995, p. 11) admonishment to not "worry about whether [participants are] 'thinking'. Just try to come to terms with how it is that the thing comes off". As is demonstrated in this section, some conversation analysts argue that not only is appeal to cognition sometimes useful (Sanders, 2005), it is actually inescapable (Deppermann, 2012).
} 
developmental psychology concerning young children's capacity to make and understand offers and requests might help conversation analysts to determine whether an observed behaviour is likely to have been an offer versus a showing. There are, therefore, a few different ways that experimental and conversation analytic research are argued to be informative to each other.

It will be noticed that the research reviewed above all focuses on the linguistic/verbal modality in social interaction. In recent years, research with autistic people has begun to branch out to study other aspects that might be different in the experiences and behaviour of autistic people. Of interest here is the increasing interest in the sensation of touch, and specifically of touch as it is used/experienced in social interaction. Research on this nonverbal interactional modality will be reviewed next.

\section{Autism and Social Touch}

Autism has largely been considered a condition of the mind. This focus on the mind has occurred even though embodied sensory and motor differences/difficulties have been reported by autistic people in autobiographical reports (e.g., Grandin, 1992). There has been a lot of experimental research backing up the autobiographical reports suggesting that autistic people's sensory experiences (Kern et al., 2006) and their motor control (Fournier, Hass, Naik, Lodha, \& Cauraugh, 2010) are different from those of non-autistic people. This has led people to suggest that these embodied aspects should be considered central to autism (Fournier et al., 2010; Leary \& Hill, 1996), or even that the social features of autism might be, at least in part, explained by these sensorimotor differences (Hannant, Tavassoli, \& Cassidy, 2016).

Touch specifically has received attention as a sensory modality that is impacted in autistic individuals. Self-report studies of autistic adults (Lundqvist, 2015), parental selfreport of autistic infants (Foss-Feig, Heacock, \& Cascio, 2012; Silva, Schalock, \& Gabrielsen, 2015; Ben-Sasson, Soto, Martinez-Pedraza, \& Carter, 2013), observational studies of autistic infants (Baranek, 1999), and experimental studies (Blakemore et al., 2006; Cascio et al., 2008) demonstrate that autistic people experience tactile stimulation differently from non-autistic individuals. The nature of this difference is debated, with different studies showing systematic hyper-responsivity (Blakemore, et al., 2006; Cascio, et al., 2008) and hypo-responsivity (Foss-Feig, Heacock, \& Cascio, 2012). As well, these differences in tactile sensation are not general within autistic individuals; different kinds of touch at different locations produce different profiles of response in autistic people compared to non-autistic 
people. For example, Cascio et al. (2008) found autistic participants were hyper-responsive to vibrational touch on their forearms compared to their palms and Blakemore et al. (2006) found that autistic participants were hyper-responsive to high frequency vibrational touch but had a similar response to lower frequency vibration as non-autistic participants. In sum, research suggests that different tactile sensitivity is a common feature of autism.

Not only do autistic people appear to experience touch differently from non-autistic people, it is argued that this sensory difference is related to autistic people's social differences and/or difficulties. Lundqvist (2015) found that touch hyperresponsiveness ratings mediated social difficulties ratings, and Mammen et al. (2015) found infants' avoidance of touch at nine months of age predicted parent-reported autism-related behaviour at 18 months of age. Neurological studies have found a reduction in volume of the nerve fibres thought to conduct signals for social touch in a small sample of autistic children (Silva, \& Schalock, 2016), as well as abnormal perception of touch and pain in body areas more highly innervated by CT-afferents compared to those less so (Riquelme, Hatem, \& Montoya, 2015). Cognitive neuroscience studies have indicated that regions of the brain thought to be associated with social-emotional information processing show reduced response to stimulation of CTafferents in autistic people's brains than in non-autistic controls (Kaiser et al., 2015), and also that autistic individuals' brains produce electroencephalographic makers of vigilance in response to perception of social touch and this signals correlated with their reported aversion of touch (Peled-Avron, \& Shamay-Tsoory, 2017). The results of these studies suggest that not only do autistic people experience touch differently from non-autistic people, their experience of touch is strongly related to their experiences of and abilities in social interaction.

Considering this evidence for autistic people's different experience of touch, it is no wonder that autistic children may avoid touch or only engage in touch under certain conditions. It has been reported that autistic children engage in cuddles less than non-autistic peers (Baranek, 1999). As well, parents report that they struggle with expressing affection to their autistic children's aversion to touch (while recognising those same children can be physically affectionate on their own terms; Cullen, \& Barlow, 2002), and parents generally rate their autistic children as less cuddly than others (Vostanis, et al., 1998; The BASIS Team et al., 2012). What these results suggest is that autistic people's different, and commonly aversive, experience of touch is not just co-occurring with social difficulties, it contributes to them behaving in ways that appear disordered or different from others by their parents. 
Aversion to social touch is commonly understood as a consequence of physical differences in touch sensitivity in autistic people. Autobiographical reports from autistic people describe their sensory sensitivity in general as something that can impede typical behavioural engagement with activities or people (Jones, Quigny, Huws, 2003; Grandin 1992). As well as this, the link between touch sensitivity and social touch aversion is strengthened by neuroscientific research into the nervous pathways and brain regions claimed to be involved in social touch, with this research showing that autistic individuals have abnormalities compared to typical individuals in these anatomical domains (Cascio et al., 2008; Silva, \& Schalock, 2016). To date, there have been no conversation analytic studies that have focused on interpersonal touch in the social interactions of autistic people. Chapter Five will address this by analysing interpersonal touch between autistic children and their family members, describing how the touch is managed within, and as locus for, social action.

\section{Summary}

Descriptions of autism have tended to emphasize social behaviours. As psychology at large adopted an understanding of social cognition as abstract cognition directed towards ostensibly social things, theories of autism's causes tended to focus on asocial psychological elements. Despite this, studies of language and autism have contributed a lot in describing the behavioural nuances of autism. In this chapter, I have reviewed some of the literature that has suggested linguistic differences in the way autistic people talk, primarily focusing on pragmatic differences and difficulties. Most importantly for this thesis, I also reviewed the research literature on autistic people in social interaction that uses naturalistic data and employs conversation analysis to examine autistic people's interactional behaviour in situ. As well, I described some of the recent research into autism and social touch, highlighting this as something that multimodal conversation analysis can contribute towards. Having reviewed the state of the art in autism, language, and social interaction research, the next chapter will describe the methodological issues involved in conduction conversation analytic, discursive psychological research, as well as the procedures followed for this thesis.

\section{Chapter Three:}

\section{Doing Discursive Psychology: Data Collection, Transcription and Analysis}

“... there is no reason to look under the skull since nothing of interest is 
to be found there but brains."

(Garfinkel, 1963, p. 190)

As the founder of ethnomethodology, Garfinkel argued that social action could be understood by looking at it happening in the contexts in which it naturally occurs. To him, one need not look into the mind or the brain to understand how behaviour is organised. In this chapter, I will outline the methods and practical considerations by which this kind of research is conducted. The first half of the chapter will discuss the use of naturalistic data in social science research and outline some of the considerations to be made in determining what to record and how to do so. Then transcription of talk and embodiment will be discussed, leading into how to analyse the transcripts and data using conversation analytic principles. In the second part of the chapter, I will outline what decisions I made in the collection and analysis of data, based on what was discussed in the first half. The participants will be described as well as ethical considerations and the storage of the data. Finally, the phenomena collected for study will be briefly introduced.

\section{Observation and Naturalistic Data in Social Sciences}

Observation is a key aspect of all scientific investigations. In order to analyse some object or phenomenon, researchers must have made some observation of that object or phenomenon's features. In some fields, what this observation consists of, how it is done, and what it provides, is more or less obvious. For example, in order to study how the brain's activity changes in response to certain perceptual stimuli, one would observe the brain in some setting where that stimuli is present, and the data would be constituted by, for example, measurements of blood oxygen levels in regions of the brain. While psychology has generally adopted quantitative approaches like experiments and psychometric questionnaires for its observation and data collection (something that has been criticised by psychologists since the 1960s; Rozin, 2001; Baumeister, Vohs, \& Funder, 2007), there has always been a tradition of observing social activity in action (naturalistic observation) and recording that as data for analysis (naturalistic data).

Naturalistic observation involves observing what people do in their everyday lives. This kind of observation has looked different across time. An early example of naturalistic observation is Roger Barker's project during which he and a team of researchers took shifts observing and recording the activity of a young boy across one full day, from waking to 
going to sleep (Barker, \& Wright, 1951). The researchers followed him everywhere taking regular notes on his activities, including his own behaviour and that of those he interacted with. The result of this was a highly detailed account of what happened during that day.

Observation of social activity in its natural setting can result in a wide variety of data. For example, Barker, and Wright's (1951) work produced a data set that consisted of the researcher's descriptions in simple sentences of the child's behaviour. In the specific domain of parent/child social interaction, Lytton (1971) reviewed different data generated from observational studies of family members interacting with each other, specifying that these data can be from ranked more abstract representations of behaviour to more concrete. His review provided this hierarchy (abridged here as it is just for demonstration); after-the-fact ratings of behaviour, narrative summaries, categorising behaviour by predetermined codes, and specimen recording like in Barker and Wright. What I will call naturalistic data is absent from his discussion.

Naturalistic data consists of audio or video recordings of actual behaviour as it happens and is contrasted with contrived data, or data which is derived from artificial settings such as interviews and focus groups (where some report of prior action/thoughts/et cetera is given or coded as data) or experiments (where the setting in which a behaviour is observed is produced by the researcher; Potter, \& Shaw, 2018). The term naturalistic is used rather than natural data in order to address an epistemic caution required (Potter, \& Shaw, 2018); naturalistic data (contrasted with contrived data) is not to be understood as recording perfectly natural social action, but is instead to be understood as data collected in ways that prioritise, and attempt, the preservation of natural social action.

Potter and Shaw (2018) suggest two tests against which data can be assessed to determine its status as naturalistic data. These are the unwell social scientist test and the recovery of action test. Data that passes the unwell social scientist test is data that records activities that the research participants would have engage in even had the researcher not set up recording equipment or was not present. Interviews and focus groups, surveys, and experiments do not pass this test (i.e., they produce contrived data). Data that passes the recovery of action test is data that an analyst could use to reproduce the actual activity that the participants were engaged in. Narrative accounts and behaviour codes, for example, fail this test as one cannot use the account of the codes to preproduce that actual behaviour that participants produced (i.e., it misses details relevant for the ordering of action like speech 
delivery and the exact sequentiality of talk and gesture). Audio and video recording of actual behaviour do pass this test as they preserve the behaviour itself rather than preserve an analysts' description/gloss of it. Naturalistic data is important if social scientists aim to produce descriptions of what people do and how they do it.

Beyond the fact that naturalistic data attempts to preserve naturally occurring action as it happens, it also has other benefits to social science researchers over more abstracted data. Lomax and Casey (1998) summarise these benefits as follows; natural data collection is adaptable to most research settings, it provides a richness and complexity of human life that analysts would struggle to record otherwise, data is reliable as it can be replayed exactly and shared to other researchers for their own analyses. As well, Potter (2002; Potter, \& Shaw, 2018) include as benefits that naturalistic data remains free of analysts' own categorising and theorising, inferences concerning behaviour (meaning, motive et cetera) are reserved for analysis, it does not require that analysts nor participants are put into the third person position of disinterested reporters of social facts. As well as these, there are benefits resulting from the use of video recordings as the primary data such as the capacity to zoom in and out and edit digital video to juxtapose scenes from different times in the video (Heath, Hindmarsh, \& Luff, 2010). Having described here the value of naturalistic data, the next section will elaborate on some of the things researchers need to take in mind for collecting naturalistic data for conversation analysis.

\section{Considerations Made in Collecting Naturalistic Video Data}

Mondada (2006) states that if the point of analysis is to produce an endogenous account of how social interaction unfolds, then the video recordings of those interactions need to make available for analysis all the features of the local ecology of that interaction that were available for analysis by the participants in the moment. In order to capture all these features, researchers need to consider at least three different dimensions in making their recordings; timing, participation frameworks, and multimodal/embodied conduct (Mondada 2006). In considering timing, researchers need to consider capturing complete courses of actions from beginning to end which poses analytic issues concerning when a course of action has begun and ended as well as material concerns around battery life and tracking courses of action as they begin and end in different settings. In considering participation frameworks (i.e., the ways that people are organised with regard to each other in order to display their mutual participation in interaction; Goodwin, 2000), researchers should attempt to record all relevant parties to a course of interaction as well as their organisation with 
regard to each other and to other objects that impinge upon how the interaction unfolds (i.e., furniture, objects like books, phones, computers et cetera). In considering the multimodality of conduct, researchers should attempt to capture features of interaction beyond the linguistic including eye gaze, touch, expressions or other gestures.

While the above are aspects the researcher should orient to in the design of video data collection, considerations around video recording should be made with respect to how the recording might impact the action being recorded, as well as how it might impact the participants involved in terms of their comfort, privacy, and autonomy. There has been debate over if, and to what extent video recording of research participants impacts their behaviour (Lomax, \& Casey, 1998). Duranti (1997) discusses how video research, like any other context in which human actors understand they are being observed/recorded, is susceptible to the participant-observer paradox; in recording participants to try understand their natural behaviour, researchers introduce things which the participants might change their behaviour around. As it would be unethical to record participants without their knowledge, researchers only aim to minimise their impact on their research participants/setting.

However, both Duranti (1997) and Mondada (2006) direct researchers not to see the camera and participants' orientations to it as contaminating the data, but instead to see the camera as a resource that participants have for constructing their action. References to the camera can highlight actions that participants orient to as embarrassing, for example (Mondada, 2006) and in Duranti’s (1997, pp.118-119) words “even with a lens aiming at them, participants still manage to argue with one another, be overrun by emotions, reveal intimate aspects of their private lives, or engage in length evaluations of the private lives of other people". This is further evidenced in my data as participants happily shared interactions that involved disagreements or fights, for example. As well, conversation analytic research has shown how children's orientations to the camera, while common, are not to be seen as contamination of the data but instead as constituting the data as evidence of how children use the camera and the fact of their being recorded in accomplishing the activity that they are momentarily engaged in (Barriage, \& Searles, 2019; Sparrman, 2005). While the camera may not have been there had the researcher not placed it there, the issue of reactivity to the camera is live in all cases where participants know they are being recorded (i.e., in family home videos, et cetera) and the issue for the analyst is to understand how it impacts the different courses of action being captured. 
As well as considering the analytic requirements data is collected for, researchers need to consider the practical implications of video recording participants in the private lives. Researchers need to consider how they will manage participants' comfort, privacy, and autonomy. This means making decisions about whether the researcher will use a roving camera versus a fixed camera. A roving camera means being able to follow action as it shifts within a much larger arena (Heath, Hindmarsh, \& Luff, 2010), however it also means the researcher is likely to be following the action and always delayed (Laurier, \& Philo, 2006) and also obviously requires that the researcher be present in the research setting. The researcher being present means the researcher becomes inevitably part of the research setting (Lomax, \& Casey, 1998) but it also means the participants do not have a boundary of privacy. If the researcher is present it means they are likely to be party to things the participants may not wish made public (e.g., family disputes or discussion of sensitive family matters). Some possible affordances of using fixed, multiple cameras (to capture as much of the setting as possible) include that the researcher does not necessarily have to be present and the participants can select or censor what is shared for research, preserving some degree of their privacy and autonomy over how they are presented. This latter approach to collecting data by leaving participants in control of what is recorded, and when, is recommended and discussed by Laurier and Philo (2006) and has been used in conversation analytic studies of autism (Wootton, 1999; Sterponi, \& Shankey, 2014; Rendle-Short, Wilkinson, \& Danby, 2015).

\section{Transcription of Talk and the Body}

Conversation analysis requires detailed transcripts of conduct to be developed. In fact, the process of transcription is commonly thought of as being the first step to analysis (Heath, \& Luff, 1993, p. 309), as decisions made regarding the representation of conduct on the transcript are themselves analytic comments on that conduct (ten Have, 2007). Analysis proceeds from transcripts rather than just reference to the recordings themselves because transcripts allow the totality (or at least extended pieces) of the interaction conduct to be seen at once. Whereas talk or behaviour only exists for inspection in the moments it is occurring, transcripts allow the conduct to persist allowing analysts to see an extended picture of the sequential organisation of the conduct in question rather than just individual moments (Hepburn, \& Bolden, 2017).

The transcripts are produced in meticulous detail, such that many aspects of the production of conduct are captured. This meticulous detail is required as it is largely impossible to know before analysis which aspects of the local ecology and the conduct within 
it will be significant for comprehending how action unfolds (Heritage, 1984). To this end Gail Jefferson initially developed the basis of the conventions used by conversation analysts for transcribing the production of talk. These conventions are designed to help analysts capture the temporal or sequential ordering of talk, including marking when talk by two or more speakers is produced at the same time and silences where no talk is produced, as well as information about intonation, speed, or vocal quality. The reason for such detailed transcription is that nothing in interaction can be presumed to have no significance to the interactants (Heritage, 1988). As such, as much of the way talk is delivered is transcribed on the basis that anything could be a resource used by social participants in building action. These conventions have been adapted and expanded upon generally (Hepburn, \& Bolden, 2017), and more specifically to include specific conventions for transcribing conduct like crying (Hepburn, 2010). The conventions used in the analytic chapters of this thesis are included as Appendix A.

As video recording equipment has become more accessible and video recording of copresent social interaction has become more common, conventions for transcribing embodied conduct and not just talk have been developed. Different methods for transcribing embodied conduct have been developed according to what specific embodied conduct is being analysed. For example, Goodwin (1981) developed a simple transcription convention for marking the movement of eye gaze and simple gestures. Lorenza Mondada's conventions (2016), however, are useful for transcribing embodied conduct more generally in multimodal conversation analysis. In this style, onset and offset of some behaviour of interest is marked on the transcript with reference to the talk with which it co-occurs. This highlights the way that talk and embodied conduct laminate each other and are thus to be understood with reference to each other. While onset and offset of conduct are marked on the lines of talk they co-occur with, the conduct itself is described on its own lines beneath the talk. In this way multiple lines of conduct can be used to track the course of different behaviours (e.g., eye gaze and gesture) as they happen at once within turns of talk. Figures (Such as image screengrabs from video clips) can also be used to supplement these descriptions of conduct. A summary of multimodal transcription conventions used are included as Abstract B, and readers are also recommended to refer to Mondada (2016) for full explication.

\section{Conversation Analytic Principles}

As described in Chapter One, conversation analysis is a method for analysing social interaction by understanding the unfolding structure of interaction from the perspective of the 
participants to it. Drawing from ethnomethodology (Garfinkel 1967), conversation analysis approaches social interaction as mutual effort toward intersubjectivity, or sense-making, such that intelligibility and order are locally produced within the interaction. Despite claims that naturally occurring spoken language are too messy to analyse (Chomksy, 1957), early conversation analysts pointed out that participants to interaction manage it just fine and by taking an emic perspective on how they do it rather than imposing the external concerns an understandings of the analysts, researchers can understand the procedures by which participants produced the order of their interactions.

Conversation analysts utilise several of key principles and techniques in engaging with data. These are unmotivated looking, participants' orientations, and the next turn proof procedure. Unmotivated looking refers to the initial phase of observation when an analyst first engages a piece of data. Unmotivated looking means engaging with the data on its own terms without concern for preconceived hypotheses or objects in mind (Schegloff, 1996). By not looking for anything, the analyst can make note of interesting phenomena as they occur in their own right, in their contexts. Sacks (1995) said that this process allowed the analyst to more easily adopt the perspective of the participants and "pose [for analysis] those problems that the data bears" (p. 471).

Adopting the perspective of the participants is another key element of conversation analysis. Conversation analysts are not interested in imposing their externalised, decontextualised under but are instead concerned with understanding an interaction from the perspective of those who were participants to it (Sidnell, 2013). This requires that analysts attend to what participants do and how what they do shows their understandings of the ongoing course of action. Participants' orientations to the local ecology an action is understood by analysts by applying the next turn proof procedure (Sacks, Schegloff, \& Jefferson, 1974). The idea behind the next turn proof procedure is that in order to see how any particular turn at talk was understood by participants analysts can look to the next turn and see how the participant responded to it as participants' responses to conduct embody their interpretation of that conduct (Garfinkel, 1967). In prioritising participants' orientations in their analyses, conversation analysts strive to use the same resources to understand the organisation of any moment of interaction that the interactional participants used (Sidnell, 2013). 
In order to demonstrate the next turn proof procedure, I will present here a short analysis of a sequence of action across three turns. Previously, Morgan has told his Mum that a kid at school has been bullying him. In this interaction, Mum is trying to talk further about this bullying.

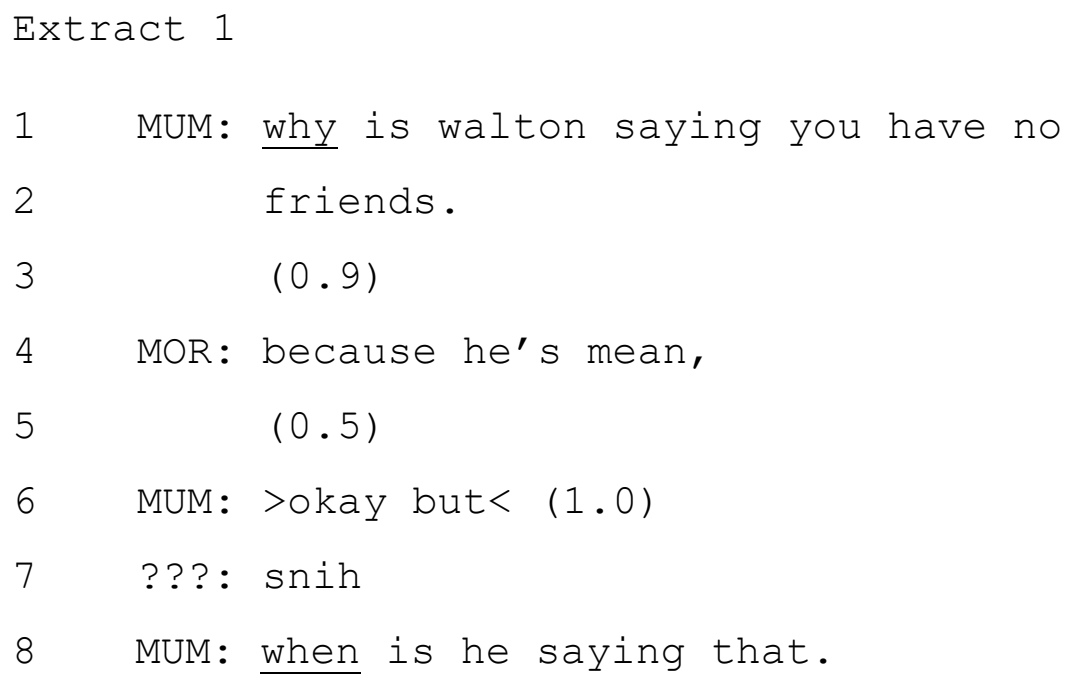

Across these three turns we can see Mum asks a question, Morgan answers it, and Mum responds to the answer. On line 1, Mum requests so information, asking what motive Walton has to say Morgan has no friends. Analysts can presume that it's a request for a motive due to the word "why" typical of initiating requests for explanations (Bolden, \& Robinson, 2011), but that presumption can be grounded and supported by looking at what Morgan and Mum do next. Morgan does not do nothing; he says something, suggesting that he understands what Mum said as something that should be responded to. His turn at line 4 is designed as a response to Mum's turn. He does this by beginning his turn with "because", which is a marker of what comes next being a reason or explanation for something (Shiffrin, 1987), making this turn fitted as a response to the turn before. As well, he uses "he's" to refer to Walton. As "he" is commonly used as a locally subsequent reference, it suggests the he refers to someone more specifically identified previously (Schegloff, 1996). Using the next turn proof procedure, it is clear to analysts that Morgan understands Mum's initial turn as a request for information (specifically an explanation) as he provides it.

While, the previous paragraph shows that Morgan understands Mum's turn as a request for an explanation, it does not show whether his understanding was correct or appropriate. Looking to Mum's turn at line 6 we can see her response to what Morgan said. Notably, Mum acknowledges Morgan's response with “okay” before immediately appending 
"but". This response tells Morgan that Mum heard him as well as indicating that it was not exactly the kind of response she was expecting. This is seen clearly in the fact that Mum then asks another question. This question on line 8 changes the question Morgan heard as being about motive into one about details of the situation that might lead Walton to say what he did. So, in this case, it can be seen that Morgan understood the question a certain way, recognised by his response to it, and that understanding was challenged by Mum, showing that it was not what Mum was originally asking for. Using the next turn proof procedure, analysts can understand how an interaction unfolds using the same resources that the participants did to conduct the interaction in the first place and analytic claims can be grounded in the data itself.

As segments of talk are selected and are subjected to sequential analysis, phenomena of interest are noticed. These phenomena may be single turns or sets of turns or they may be entire extended sequences. These may be treated differently with some phenomena gathered together in a collection of similar cases, and some treated separately and independently as case studies (Hutchby, \& Wooffitt, 2008). For this thesis, I developed two collections and one extended case study analysis.

The purpose of developing collections of similar cases of a phenomenon is to be able to highlight that similarities and differences between individual instantiations of the phenomenon (ten Have, 2007). While the cases in a collection may all be exemplars of the same kind of thing (for example, accounts), they will vary on the specific details of their occurrence due to their specific, local position in different courses of action. As such, collections of an interactional phenomenon resemble a naturalist's collections of biological specimens where such a collection of specimens allows for the systematic study of the possible elements in common and/or variations within the domain of interest (Heritage, 1988).

While collections are important for understanding the variety of way is particular action can be accomplished in the different interactional locations it may appear, it is important to remember as well that, as Schegloff said, "There is a constitutive order to singular occasions of interaction" (1987, p. 102). In any given moment, participants make sense of what is occurring around them and in their interactions and they do this without reference to collections of phenomena. Order and action are managed on a moment by moment basis, and as such, single, extended cases of interaction can be analysed in order to 
explicate this particularly local construction of order. Not only is the analysis of cases studies important for understanding local intersubjectivity, it is important to conversation analysts as a way of validating the findings of previous conversation analytic work (Schegloff, 1987). In this way, previous conversation analytic findings function as keys into the analysis of the moment at hand and they can be evaluated with reference to how well they seem to "unlock" for the analyst what is going on. If some previous finding does not seem to hold for the analysis in question that might suggest that the moment under current consideration is of a different nature than previously thought or suggest some limitations to the previous analysis.

\section{Method for this Thesis}

\section{Participants}

Participants were recruited with a flier that was distributed online (primarily on Facebook) initially by a local autism family support group and then by others who shared the posted flier around their own social networks. At the outset, I aimed to recruit families/households where any member had received a formal diagnosis of Autism Spectrum Disorder. The flier briefly said that research participation would involve families/household video recording themselves as they engaged with each other around the home and that interested parties could contact me via email.

Ultimately, four families contacted me. Each of these families made contact primarily because their children had been diagnosed with Autism Spectrum Disorder and as such the project became selectively concerned with child peer and parent-child interactions. One family had two children (Morgan and Aidan, aged ten and six), another had one child (Trevor, aged six), another had two children (Magnus and Olivia, aged ten and six), and the last had two children (Raphael and Cecilia, aged three and seven). Olivia was the only child not to have received a diagnosis of Autism Spectrum Disorder. All names used are pseudonyms. In two of the families one of more parents had also received an Autism Spectrum Disorder diagnosis (a mother from one family and a father from another family) and another mother had received a tentative suggestion of membership on the autism spectrum. All of the families were Pākehā/European speakers of New Zealand English (except for one family, the parents of which were both from the United States of America).

Specific details about the diagnostic assessments and the children's performance on standardised tests are not reported here. The reason for this is that that kind of data plays no role in the analysis of local social action as conducted here through conversation analysis. 
This means that any behaviour analysed in this thesis cannot be compared against a documented tendency to act in any sort of way, however conversation analysis aims to explicate how any individual behaviour shapes, and is shaped by, the local interactional context in which it is embedded.

\section{Ethics}

The project was approved by the Victoria University of Wellington School of Psychology Human Ethics Committee (Ethics Application ID: 0000025632).

I met with families to discuss the project, what would be required of them, what I intended to do with the data and how their privacy and security would be maintained. Both parents and children were present, but children were not always engaged with the discussion. This was not unexpected. All parents committed to participating during these discussions however I asked that they discuss participation in the project with their children using an informed assent form I designed for the children while I was not present having left the cameras with them. This way the parents and children could discuss on their own terms without my being there. When I returned to collect the cameras, I also received signed copies of the childrens' assent forms and families were given a last chance to look over the recordings they were sharing and withhold anything if desired.

All recordings were saved on a private and secure storage server at the university. Only myself and my PhD supervisor had direct access to this storage. As well, recordings were saved onto an encrypted external hard drive that only I had access to. This was done for redundancy in the case of trouble accessing one or the other source.

In two recordings, it was made clear by the children that they did not wish to be recorded. In one of these recordings, the parent managing the camera said they were not recording. This raised an ethical issue regarding use of the video. Due to their age, the children involved in this study could not give informed consent. As such, their parent's gave consent for them and the children provided their own additional assent. Despite this, the children's clear lack of assent to be recorded was considered over parental consent and these recordings were deleted.

Consent was not sought for just collecting recordings for analysis. Families were also asked if they would allow me to use non-anonymised images and video extracts in publications and conference presentations. All data is stored separately from records that name, locate or otherwise identify participants and participants places are pseudonymised in 
transcripts, however the video clips themselves are stored in an unaltered form. Participants were given the option of allowing me to use non-anonymised material, or anonymised material Anonymised material would mean that the images and video clips would be altered such that identity could not be derived from their faces. This would be done by applying a filter with video editing software over the image or video that would mask the face alone or the whole frame. An example of this would be a filter which highlights visual edges producing an image like a line drawing or sketch that would retain an image of the action while reducing identifiable detail. If participants did not consent to me using any material in publications of conference presentations, they were not included in the study. This decision was made to ensure that all data would be available in some way for inspection by readers of publications or attendees at conferences. All families excluding one consented to use of nonanonymised materials.

\section{Data Collection}

I visited each family at their homes to further explain how data collection and the project more generally would work. If they then consented to participate, I left them with two video cameras and asked them record anything they felt comfortable with sharing with me for research. I gave them suggestions of things like family mealtimes, game times, morning and bedtime routines and anything else they could think of. Together, we selected a location in the home that would serve as the primary recording area. In all cases this ended up being their living rooms. We agreed on locations for the cameras to be positioned in, but I also informed them they could remove the cameras to other sites as desired. I asked them also to have both cameras recording at the same time as much as possible. Originally, approximately ten hours of video was recorded across all families, however due to the considerations discussed below, eight hours of video comprised the final corpus. The amounts of video and brief selections of content recorded are described in Table 2.

Table 2

Amounts and brief description of video recorded.

\begin{tabular}{lcl}
\hline & Amount of video (hours) & $\begin{array}{l}\text { Selection of content } \\
\text { recorded }\end{array}$ \\
\hline Morgan and Aidan's family & 2 & $\begin{array}{l}\text { Preparing for/returning from } \\
\text { school, planning activities, } \\
\text { talking about media }\end{array}$ \\
Magnus and Olivia's family & 0.4 & $\begin{array}{l}\text { Dinner time, preparing for } \\
\text { school, planning activities, } \\
\text { preparing dinner }\end{array}$
\end{tabular}


Raphael and Cecilia's family

Trevor's family
2.6
Dinner time, playing with parent, playing together, preparing food

Dinner time, playing with parent, preparing for bed, opening presents, talking about media

In considering the impact of recording on the participants' comfort, privacy, and autonomy, I made the decision to give participants ultimate control over the cameras and what they record as well as control over what they shared with me for analysis. With my absence, not only did I minimise the impact of the researcher on the natural unfolding of their social interaction, I also gave the families the space to select what they were comfortable with me seeing, giving them privacy and control to exclude for analysis anything they would prefer not be seen outside the family.

In this study, families were given video cameras to turn on and off as they wished. As a result, the video collected was not as clean as it would have been had I stayed and managed the recording myself. For example, parents had difficulty with the two cameras and frequently only set one recording thus some action was missed due to the single viewpoint. As well, cameras were often started at times when action was already ongoing or turned off before courses of action that had been begun were recognisably complete and thus beginnings and ends were not recorded. This means individual recordings were of wildly different lengths, with some as short as five minutes and others as long as 20-30 minutes (one of the families provided video of them watching a movie together as a family which lasted approximately 2 hours, however was not included for analysis as their action was minimal and when it occurred required the movie footage to be comprehensible). However, within the longer recordings there are whole courses of actions captured and transcription and analysis ultimately focused on those scenes.

Regarding participant frameworks, the home positions that I and the participating families agreed upon were chosen as an attempt to capture as much of the action within a certain space as possible. Families were also given two video cameras in order to capture action from more than one perspective. Sometimes the parents would shift the cameras in order to better capture the relevant action. While individuals would move in and out of frame sometimes during the course of recording, there are full courses of action during which all participants were on screen and transcription and analysis focused on these moments. 
Regarding multimodality, as mentioned above, families were given two video cameras and they were initially set up such that action could be recorded from two perspectives, maximising the multimodal detail captured. This allowed for me to see what participants might be doing even when one camera view was obscured. Ultimately this was also hindered by whether or not parents would actually use both cameras. Many of the recordings were made with one view. Analysis prioritised recordings of moments where all relevant conduct was visually available. Recordings of moments where this was not the case were not excluded from analysis but were used as support for other analyses rather than standing tentatively on their own.

\section{Data Management and Selection}

At the end of the recording periods, I met with the families again to discuss their experiences of recording, to check whether there was anything they recorded they did not want to share with me, and to take back the camera equipment. As well, streaming the long high-quality videos from the server for analysis was strained and resulted in lag and so primarily analysis was done drawing on the data saved on the external hard drive as recordings could be played directly without difficulty.

Recordings were not stored anywhere near identifying information. The identities of participants were only recorded on the consent forms signed by them. All participants were given pseudonyms, and these were described on a .txt file in the folder associated with each family's recordings. Recordings were labelled anonymously and generically. Video labels took the form AUT-XX-YY. AUT here refers to the autism in family interactions project, the $\mathrm{XX}$ is a number from 01 to 04 indicating the family the recording is from, and the $\mathrm{YY}$ is another two-digit number identifying the specific interaction recorded. In the case of the same interaction recorded with two video cameras, an additional $\mathrm{C} 1$ or $\mathrm{C} 2$ was indicated after the main identifier to denote which camera/view a recording was from.

\section{Workflow}

Once recordings were stored, I watched through all the videos as I received them in order to get a sense of what kinds of social occasions were recorded. The recordings were of a wide variety of activities within the home and no specific contexts were initially selected to focus on for analysis. Instead, I began transcribing interactions that most easily and obviously appeared to be complete/contained courses of action, focusing first on the recordings that managed to capture all relevant participants in shot. After transcribing a portion of the total 
corpus, I began to notice interactional moments that I thought warranted further analysis as well as noting phenomena that become the basis of several different collections of interactional phenomena. For example, I began to form collections of explanations or accounts as well as a collection of physical embraces. As I continued transcription I would add to these collections and ultimately focused my transcription effort to courses of action that contained the phenomena of interest (these collections are described further in a later section). As a result of this process of starting wide and then homing in as the phenomena of interest became clear, not all of the data recorded was transcribed (about 2 hours), however all of what has been transcribed has been done in extensive detail and much of it also contains some degree of multimodal transcription as well allowing for in-depth analysis of many interactions.

The workflow for transcription is depicted here (Figure 1). Transcription proceeded with a Microsoft Word document open as well as the recording open in two forms; in VLC media player, and Audacity (sound editing software). Initial transcription primarily focused on the talk and simple annotation of features of its production. Commonly, transcription of talk would involve the repetitive listening of short moments in order to ascertain points of overlap onset/offset or intonational contours. The video was viewed alongside initial transcriptions in case some embodied component seemed particularly significant (e.g., when talk continued while leaving the room, or was not hearable due to other activity cooccurring).

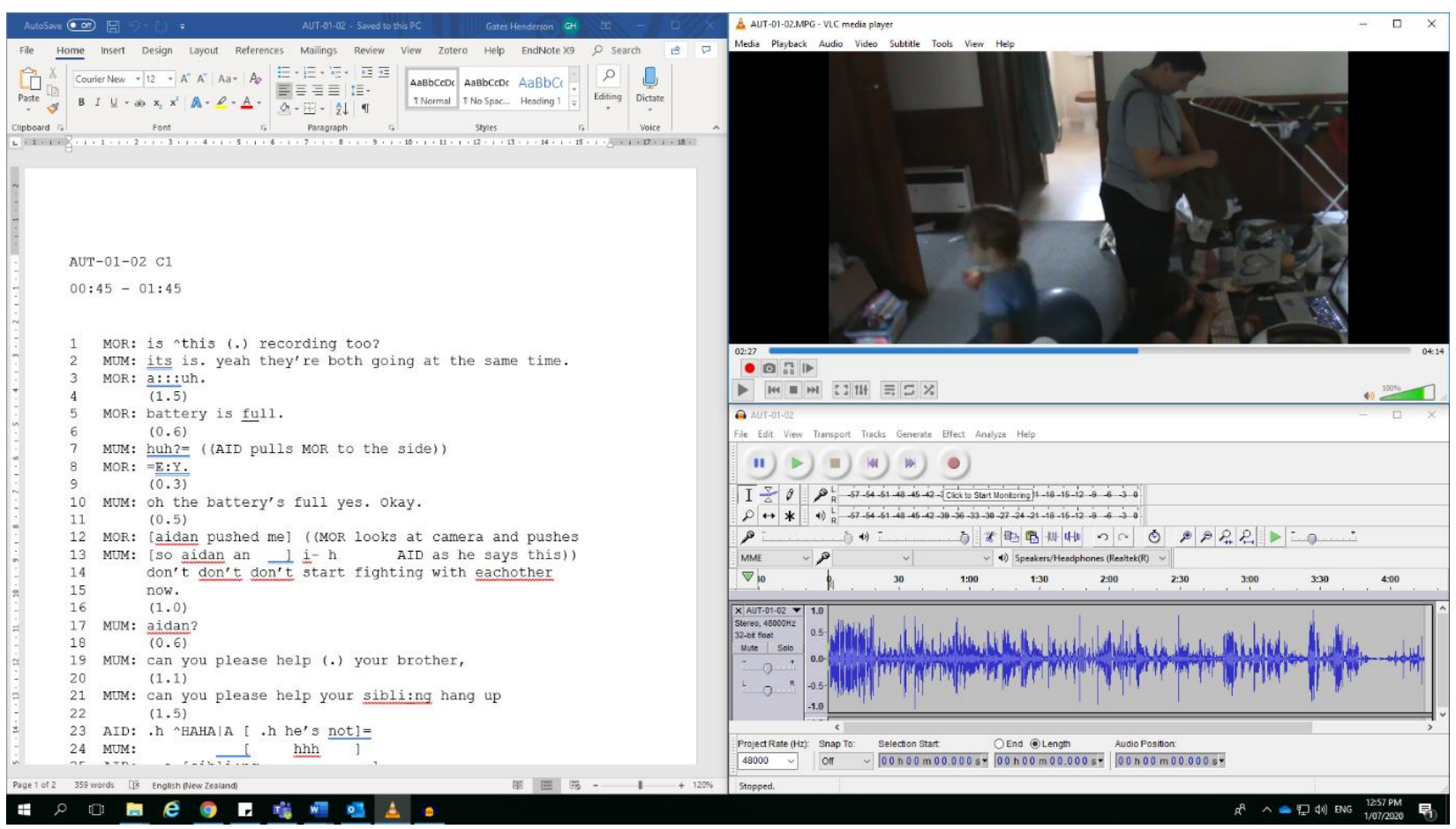


Figure 1. Workflow setup with the transcript (left), the video clip in VLC (top right), and audio in Audacity (bottom right).

Transcription is not a completely objective process and the resulting transcripts are not to be understood as complete representations of events. They are partial representations of the data (i.e., the video recordings) that are designed to optimise two features; demonstrability of relevant points for the current analysis, and readability. Through the process of analysis, transcripts of even the same interactions may take different forms due to representing different features of the talk for the purposes of different analytic points. When presenting transcripts, the version of the transcript that includes only those things that are most relevant to the analysis at hand are shown in order to avoid complicated and cluttered transcripts that are hard to read. In this way, any singular transcript is a selective representation of events, highlighting some elements and likely hiding others (ten Have, 2007). Note that in the transcripts presented in the analytic chapters of this thesis, the extracts that include multimodal components will have the lines of talk bolded in the service of readability.

In the course of transcribing and analysing segments of talk I noticed certain phenomena that seemed to reoccur in spite of their appearing to accomplish things autistic children were claimed not to do or experience difficulty doing. Specifically, phenomena that would seem to be highly sophisticated demonstrations of Self- and Other-oriented social activity (e.g., considerations of others' understanding in interaction and how they themselves could be seen and treated) and physical affection by the autism-diagnosed children despite difficulty maintaining social relationships and social-emotional reciprocity being core aspects of the description of Autism Spectrum Disorder in the DSM-5 (American Psychiatric Association, 2013). While basic aspects of talk like responding to first pair parts (e.g., questions, requests, offers et cetera) with second pair parts (e.g., answers, provision/withholding, acceptance/declining et cetera) are themselves other-attentive in their responsiveness, through unmotivated looking I found complex sequences of action in which the children oriented their action towards the understanding of their interactional partners.

One of the collections I produced was a collection of 45 instances of children's accounts for their own behaviour. After the initial cases were found through unmotivated looking, further cases were found by utilising previous conversation analytic research on accounts. I looked for children's utterances that included the causal clause marker because 
and looking in positions where accounts would normally be expected based on previous literature (i.e., in dispreferred second pair parts and associated with putative dispreferred first pair parts (Antaki, 1994; Heritage, 1988).

The second major collection I developed was a collection of instances of touch. After building this larger collection I became interested in a sub-collection involving embraces, moments when either parent or child would wrap their arm, partially or fully, around their cointeractant. This collection was comprised of 12 cases. These cases were transcribed in extensive multimodal detail and analysed for the way the embrace was entered into and exited from in the midst of the courses of interaction in which they were embedded.

As well as these collections, I analysed a case of an extended interaction during which a mother directs her child to use the bathroom before bed. This moment is extended as the child continually resists, and as such the mother persists in her direction. Throughout the course of this extended sequence, various resources are deployed to elicit engagement from the child with the children deploying the same to resist, including constructions of their relationships to each other, their relative autonomy, and other embodied/postural formations.

\section{Summary}

Conducting conversation analytic discursive psychological research involves developing an understanding of what the participants are doing from their own perspective as participants in the interactions being studied. This requires critical consideration of the data that would allow analysts to build that kind of emic account of any interactional moment. Naturalistic data (video and audio recordings) of naturally occurring interactions in naturally occurring interactional settings is paramount to ensuring that accounts of social interaction do not depend too heavily on external, etic presumptions about normal or appropriate action. And because action is fundamental in social interaction, the data itself and the analytic approach must be such that it can capture and appreciate all that the participants might be able to draw upon within their interactional ecology to accomplish whatever it is that they are doing. Appropriate data (i.e., recordings that capture the full temporal and spatial unfolding of full courses of activity) and an analytic approach (i.e., sequential analysis and next turn proof) are essential. Having described what is required of a project like this, and how the research was ultimately undertaken for this thesis, the next three chapters present three analytical results. 


\title{
Chapter Four: \\ Enacting Self- and Other-Orientation by Providing Explanations of One's Own Behaviour
}

\author{
"Paradoxically, individuals with autism spectrum conditions \\ have been characterized as both impaired in \\ self-referential cognitive processing, yet also egocentric. \\ How can the self in autism be both 'absent'... \\ yet 'all too present'..." \\ (Lombardo, \& Baron-Cohen, 2010, p. 393)
}

One of the most enduring characterisations of autistic people is that they are egocentric or tend to understand and engage with other people in terms of how they relate to the self (Frith, \& de Vignemont, 2005). It has been suggested that this claimed egocentrism might derive from, or be coextensive with, difficulties in self-related cognition, resulting in a difficulty in distinguishing Self from Other (Lombardo, \& Baron-Cohen, 2010). These tendencies (either to prioritise relations to the Self, or to struggle with distinguishing Self and Other) are what is claimed to be behind autistic people's so-called social difficulties, from judging appropriate conversational contributions (Surian, Baron-Cohen, \& Van der Lely, 1996) to understanding what other people might know of beliefs (Baron-Cohen, Leslie, \& Frith, 1985; Baron-Cohen, 1991).

This Theory of Mind characterisation of autism is based on an understanding that social interaction is largely a project in attributing mental states in order to predict behaviour. A discursive psychological approach (informed by conversation analysis and ethnomethodology) instead treats social interaction as accomplished through the mutual coordination of public, recognisable action. Participants are said to be concerned not with mental states but with appropriate next responses to prior actions (Enfield, \& Sidnell, 2017). A way that participants and analysts can see this attentiveness to other's action and concerns (i.e., other-attentiveness) is through the use of accounts. This chapter will begin by briefly introducing accounting practices and then present analyses which show the autistic participants of this research deploying accounts in ways that show them to be sensitive to the local interactional factors that might impact their interlocutors' producing appropriate next 
moves. In their use of accounts, the work of orienting to self and other is woven into the unfolding of action.

\section{Accounts}

Accounting practices refer to the ways that participants make their conduct "visiblyrational-and-reportable- for-all-practical-purposes i.e., account-able" (Garfinkel, 1967, p. vii). According to Garfinkel, we build social action such that it is comprehensible according to a normative organisation. Doing so allows our social conduct to be appropriately apprehended and responded to by others. This comprehensibility is what Garfinkel called accountability. One way that people make their conduct accountable is by providing an explanation, or an account, for it. Accounts are utterances which attend to breaches of social norms or expectations by excusing (i.e., admitting the behaviour was wrong, while denying full responsibility) or justifying (i.e., accepting responsibility while denying the negative qualities of the behaviour) conduct (Scott \& Lyman, 1968).

Garfinkel (1967) and Scott and Lyman (1968) described accountability and accounting at slightly different levels. Scott and Lyman described accounts as given to repair socio-moral breaches "whenever an action is subjected to valuative inquiry" (1968, p.46). To them, accounts were given for breaches of social norms, like piling your plate high at a buffet restaurant. For Garfinkel, accounting does not just concern social norms but also the level of the interactional order as well. Accounts regularly come alongside or in response to actions (or inaction, in the case of silences and withheld responses) that have been, or could be, evaluated as inconsistent with interactional norms. In fact, the presence of an account demonstrates the speakers understanding that just that is happening. For example, accounts are common in second position as part of (or entirely constituting) a dispreferred response to some first action (Heritage, 1988). A dispreferred response is one which disconfirms or rejects an initiating action (e.g., the dispreferred response to an invitation to a party is to decline). Accounts are commonly given in dispreferred responses as the normatively accountable response is not provided, and this absence is held to require some explanation. Heritage (1988) described these accounts as commonly designed with a no-fault orientation so as to maintain social cohesion in the face of disruption to the normative order. An example of this would be the case of declining a party invitation with the explanation that you have some prior commitment (e.g., a family engagement) or inability to attend (e.g., being out of town). Additionally, accounts can be provided pre-emptively or during the normatively 
unexpected or untoward behaviour in the case of people accounting for putative dispreferred first pair parts, for example when making a request of someone (Antaki, 1996).

Although there is a large literature on adult accounting, research on accounts given by children is limited. A couple of studies have looked at both neurotypical (Hutchby, 2002; Lamerichs, Alisic, \& Schasfoort, 2018) and autistic children's (Stickle, Duck, \& Maynard, 2017) use of I don't know as an account for not answering questions and a method of resistance to questioning in therapeutic settings. The few studies on children's accounts in parent-child talk has largely focused on how accounting constitutes the socialisation of morality (with neurotypical children, see Sterponi, 2003; with autistic children, see Sterponi, 2004). For example, Sterponi (2003) showed how parents can design their account solicitations to construct the problematic event in question such that it projects certain remedial moves in the child's accounting. This work also shows that children can build their accounts oriented to the specific construction of the problematic event in the elicitation, showing that their accounts are designed for specific, local purposes and demonstrate sophisticated moral reasoning (Sterponi, 2003). Sterponi (2004) showed how the autistic children in their study could explicitly formulate other's conduct as transgressive, construct their own conduct as rule-oriented, and make complaints about others' behaviour. As well, they showed how an autistic child can be socialised into accounting through the observation of their neurotypical siblings' uses of accounts (Sterponi, 2004).

The analyses presented here will address the issue of autistic children's Self- and Other-oriented social cognition. Such cognition is respecified as displayed knowledge of, and practical reasoning about, the characterisations of one's own behaviour as those characterisations are embedded in courses of action. As well, it expands on Sterponi's (2004) work by demonstrating how autistic children's accounts orient to the interlocuters' problem of producing an appropriate next move. By looking at accounts provided in two sequential positions, these analyses will show how the children manage the characterisations of their conduct available to their interlocuters, and specifically challenge other particular available characterisations of their conduct.

\section{Analysis}

The analysis presented here will proceed by first demonstrating how the children in my sample reasoned about the causes of behaviour by explaining their own behaviour in terms of internal states and their causes. From there, I will discuss how the children in my 
sample used explanations of their behaviour to address understandings of their conduct displayed by their parents' first pair parts. Lastly, I will demonstrate the children's use of explanations for their upcoming or concurrent behaviour, pre-empting possible understandings of their behaviour, perhaps the most powerful evidence because it shows the children to be projecting a course of action before it occurs.

\section{Explanations Constructing the Link between Cognition and Conduct}

Autistic individuals often show difficulty reasoning about their own mental states as well as others' (Lombardo \& Baron-Cohen, 2010). The following two cases, however, show two children diagnosed with autism constructing their internal states. Specifically, they construct a link between their internal states and their behaviour that can provide an explanation for their behaviour. Most of the explanations collected do not refer to the children's mental states as reasons for their behaviour. Despite that, they do occur and can be understood as demonstrating the child's practical reasoning about feelings their relation to conduct in the moment.

Extract 1 occurs when Mum, 10-year-old Magnus, and 6-year-old Olivia are making dinner together. Mum notices Magnus' heavy breathing and calls him to explain it at line 8 . Just before this extract, the children were told that their cat, Errol, is unwell

Extract 1

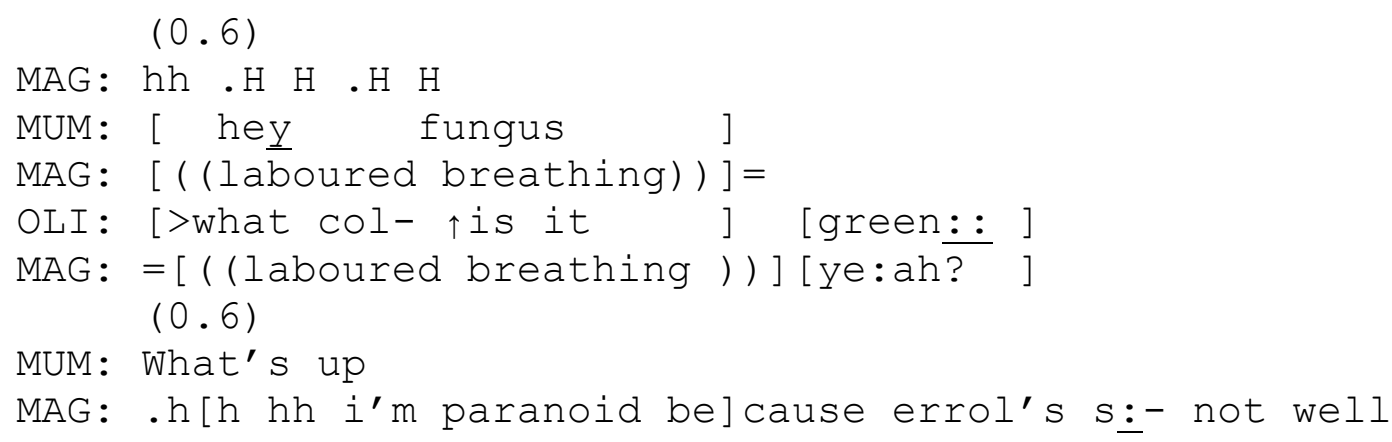

As Mum and Olivia continued speaking, Magnus left the kitchen and begins his laboured breathing, marked in line 2. Mum addresses Magnus with a pet name Fungus at line 3 and asks "what's up." at line 8, soliciting an account for his laboured breathing. Magnus provides the account at line 9 saying "I'm paranoid because errol's s:- not well.". This explanation treats Mum's utterance as if it was holding him to account for his heavy breathing (and not, for example, his leaving the room), and characterises a way that emotions and behaviour relate to each other in the intelligibility of action. His explanation is designed such that he explains his behaviour (his breathing) with a mental state term (paranoid) and 
then, in the same turn-constructional unit, he explains this mental state with an external eliciting stimulus (the previous announcement by Mum that Errol is unwell). As a result, he shows not only that he can explain his own behaviour, but that he can do so with reference to his own mental states, and that he understands his mental states need to be explained as having a cause, as well.

Extract 2 occurs at the beginning of a longer sequence in which 6-year-old Trevor and his Mum debate whether Trevor should go to the bathroom before bed. Trevor refers to mental states as well as other internal states as a basis for rejecting Mum's direction to use the bathroom.

\section{Extract 2}

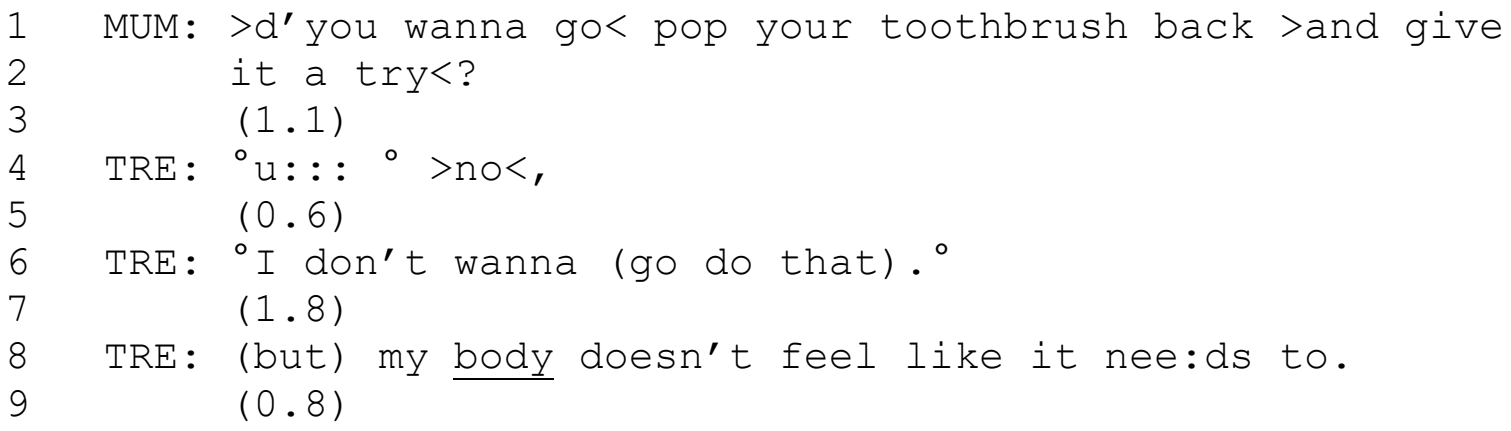

Trevor here shows that he can justify his inaction with reference to internal states, demonstrating an appreciation of the conversational norm that we have primary rights to describing our own experiences (Sacks, 1985). At line 1, Mum issues a directive to return his toothbrush and try using the toilet before going to bed. Trevor initially rejects the directive with " $\mathrm{u}::{ }^{\circ}>$ no $<$," at line 4 before giving two different accounts for his rejection, both of which refer to his internal states. The first, at line 6, refers to his desire to do what Mum has suggested by saying “o I don’t wanna (go do that). ${ }^{\circ}$. After this explanation is not taken up by Mum (shown by the long silence at line 7) a second explanation is given, at line 8 , constructing an absence of necessity. This second explanation is provided in the form of an object-side assessment. Object-side assessments attribute the qualities being commented on to the object itself, characterising the assessment as a recognition of fact rather than as the result of individual opinions or feelings (Wiggins, \& Potter, 2003). This is significant here as Trevor is positioning his compliance as specifically not a personal issue, but an objective one.

As with the case above, but less explicitly, Trevor is characterising a causal connection between internal states and behaviour. Firstly, and more generally, by giving explanations for not complying with Mum's directive that refers to internal states, he links 
behaviour with internal states, suggesting that behaviour is driven by (or at least requires) something inside people (in this case, desire and interoception). Secondly, and more specifically, in his second explanation, Trevor is referring to an absence of a feeling to explain his non-compliance. This builds an understanding that actions need some positive internal precursor (for example, a present need to urinate) behind it by suggesting that the absence of such a precursor is reason enough not to act.

The above two cases demonstrate how explicit practical reasoning about the connection between mental/internal states and their conduct is accomplished. They also demonstrate that this reasoning is conducted in at least two different interactional contexts; in response to a direct solicitation of an account, and in an unsolicited, self-orientation to the accountability of their conduct. These interactional contexts will be further discussed in the next sections.

\section{Explanations Addressing Others' Understandings Displayed in First Pair Parts}

While the previous cases showed how children utilised explanations for their behaviour that constructed a relationship between their internal, mental states and their behaviour, the following extracts demonstrate how they were able to provide explanations for their behaviour that addressed the characterisations of their behaviour constructed by others in their first pair parts.

Extract 3 occurs when Morgan and his guardian, Francesca, are in the kitchen before school. Morgan is looking for reusable straws to drink his tea with. Morgan uses an explanation for not looking where Francesca suggests that relies on a knowledge differential between them, as embodied by Francesca's suggestion.

\section{Extract 3}

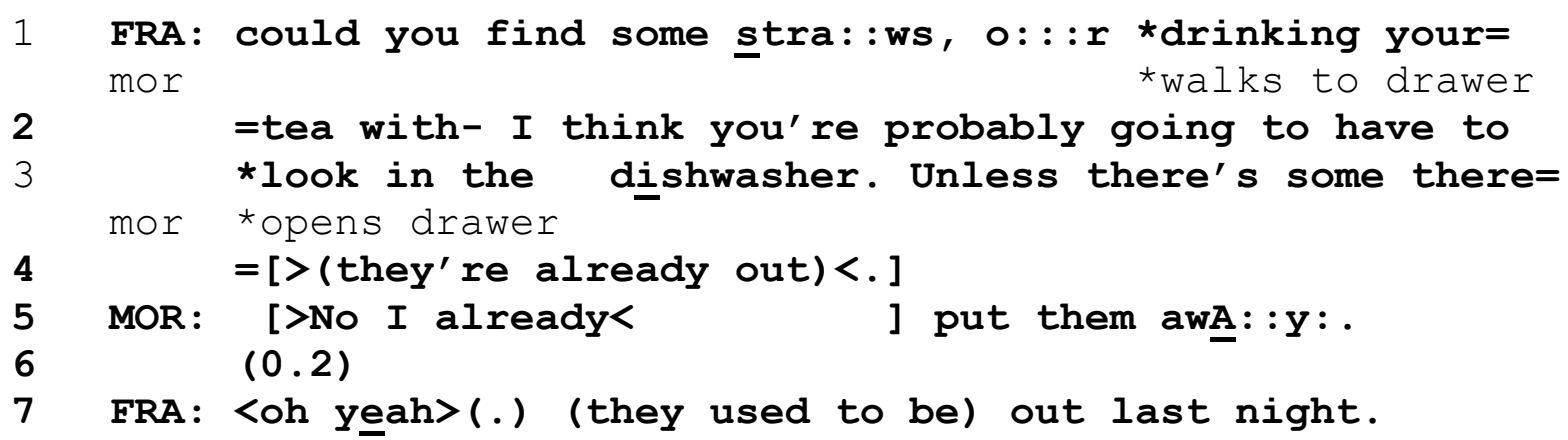

After Francesca asks him to find some drinking straws at lines 1-2, Morgan moves towards the cutlery drawer and begins to look inside. His embodied conduct constitutes his 
knowledge of the straws' location. As he begins to move towards the drawer, Francesca suggests looking in the dishwasher. This performs her understanding that the straws are not in the drawer. Heritage (2012) discusses knowledge in social interaction in terms of epistemic status and stance. Epistemic domains are characterised as territories of knowledge to which participants will have different degrees of access. This relative access is referred to as epistemic status which can be represented as lying on a gradient between $\mathrm{K}+$ and $\mathrm{K}$ (Heritage, 2012) and people display their knowledge and commitment to that knowledge by publicly taking an epistemic stance. By making her suggestion to look in the dishwasher after Morgan has already begun looking elsewhere, Francesca is taking a higher epistemic stance than Morgan; that is, she is suggesting she knows better than Morgan does.

After both parties' behaviour suggests candidate locations for the straws, Morgan responds to Francesca's utterance and its encapsulated epistemic claim and claims a higher epistemic status relative to her. At line 5, Morgan gives a dispreferred second pair part to Francesca's suggestion with and explanation. The fact that he provides one at all demonstrates his concern for how Francesca should treat his "no"; his "no" is not to be treated as purely being resistant to the directive, but as non-compliance for a valid reason. Morgan's explanation comes immediately after the "no" claiming that he has put the straws away already. By saying this rather than conceivable alternatives (e.g., "I don't want to", "I don't think so"), Morgan attends to Francesca's presupposition built into her suggestion (i.e., that Morgan does not know where the straws) are by positioning the location of the straws well inside his own epistemic domain.. In his explanation, Morgan draws on a construction of his previously acquired knowledge that puts him at a $\mathrm{K}+$ epistemic status relative to Francesca's K- status, in order to explain his conduct. This explanation directly attends to and modifies Francesca's enacted presumption of the straws location and shows Morgan's concern for her understanding of the situation.

Extract 4 occurs when Trevor is sitting at the table playing with a Lego construction while his Mum is preparing lunch. Trevor constructs the absence of a normatively expected response to his utterance from his mother as based in a lack of understanding by explaining what he said and did. 


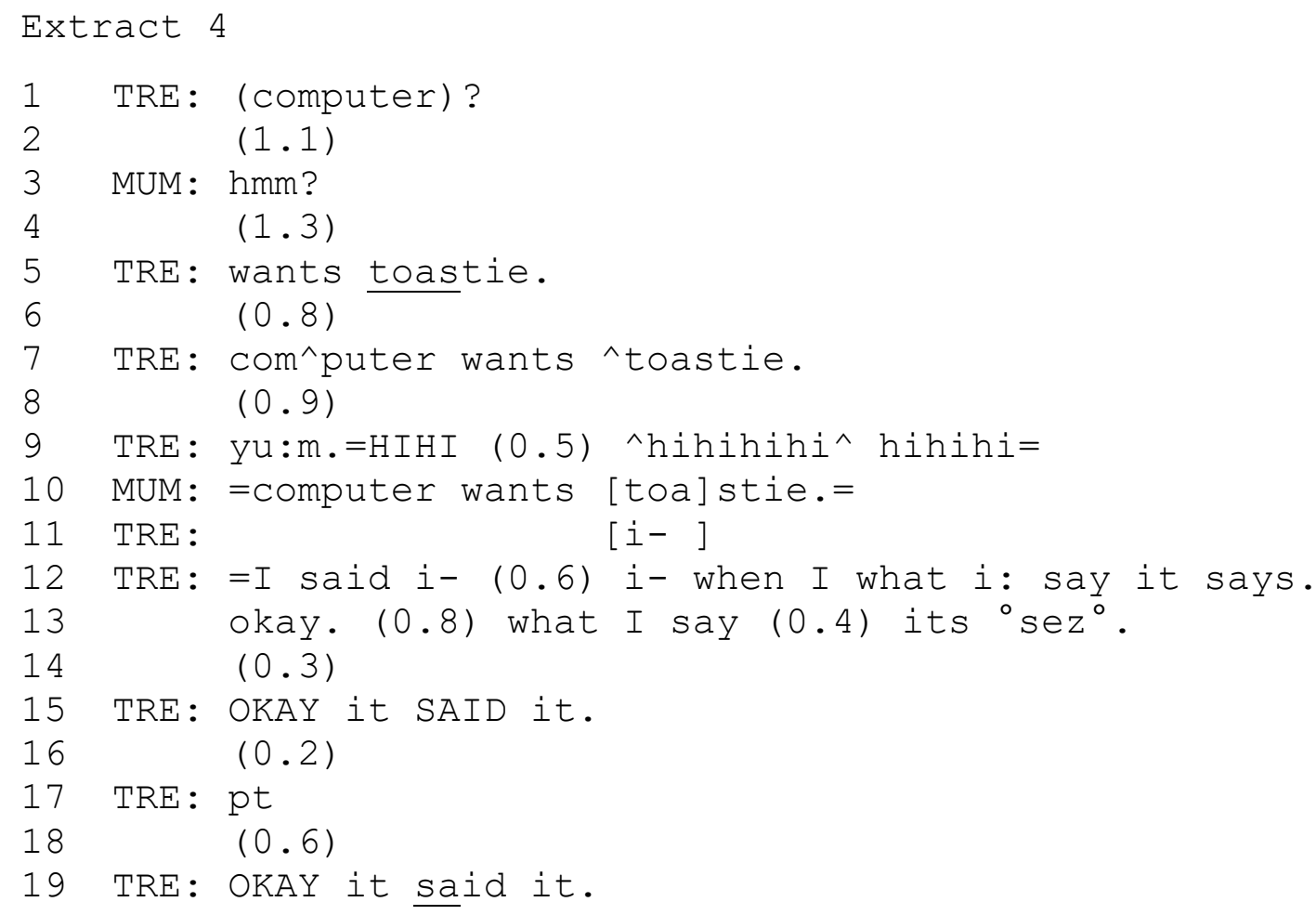

Trevor is sitting at the dining table. On the table he has a Lego contraption he has built and is pretending it is a computer. Trevor's explanation at line 12 explains how he was speaking as two different people; at lines 1 and 5 he was speaking as himself, and at line 7 he was speaking as the computer. These two voices are marked by slightly different, 'robotic' voicing on the utterance at line 7. At line 9, it is unclear if he his "yu:m" is speaking as the computer or not, but his turn ends in laughter, producing what he has done as a laughable.

At this point, Mum does not take up the invitation to join in the laughter herself (laughter after an utterance by same speaker normatively invites a second person to laugh; Jefferson, 1979). Instead, at line 10, Mum repeats what Trevor had said at line 7 . The fact that she repeats it (and thus clearly heard it correctly) yet does not join in the laughter suggests that her repetition is initiating repair, seeking clarification or further explanation (Sacks, Jefferson, Schegloff, 1977). It is at this point, at line 12, that Trevor explains that what he says the computer also says, rendering he previous repeated talk comprehensible as two different voices speaking. His explanation here shows that he treated Mum's repeat of his talk as a repair initiation. It also shows how Trevor is able to build his explanatory talk within the little game he is playing as he repeats his explanation as the computer.

The previous extracts demonstrated how participants can appreciate a speaker's analysis of the situation or their conduct as displayed in a verbal turn at talk. Extract 5 shows Morgan providing an explanation in response to an embodied move. Here, Francesca and 
Morgan are sitting next to each other on a couch and Francesca is typing in the password for Morgan's laptop. The children are not allowed to know these passwords so that their parents can regulate their technology use.

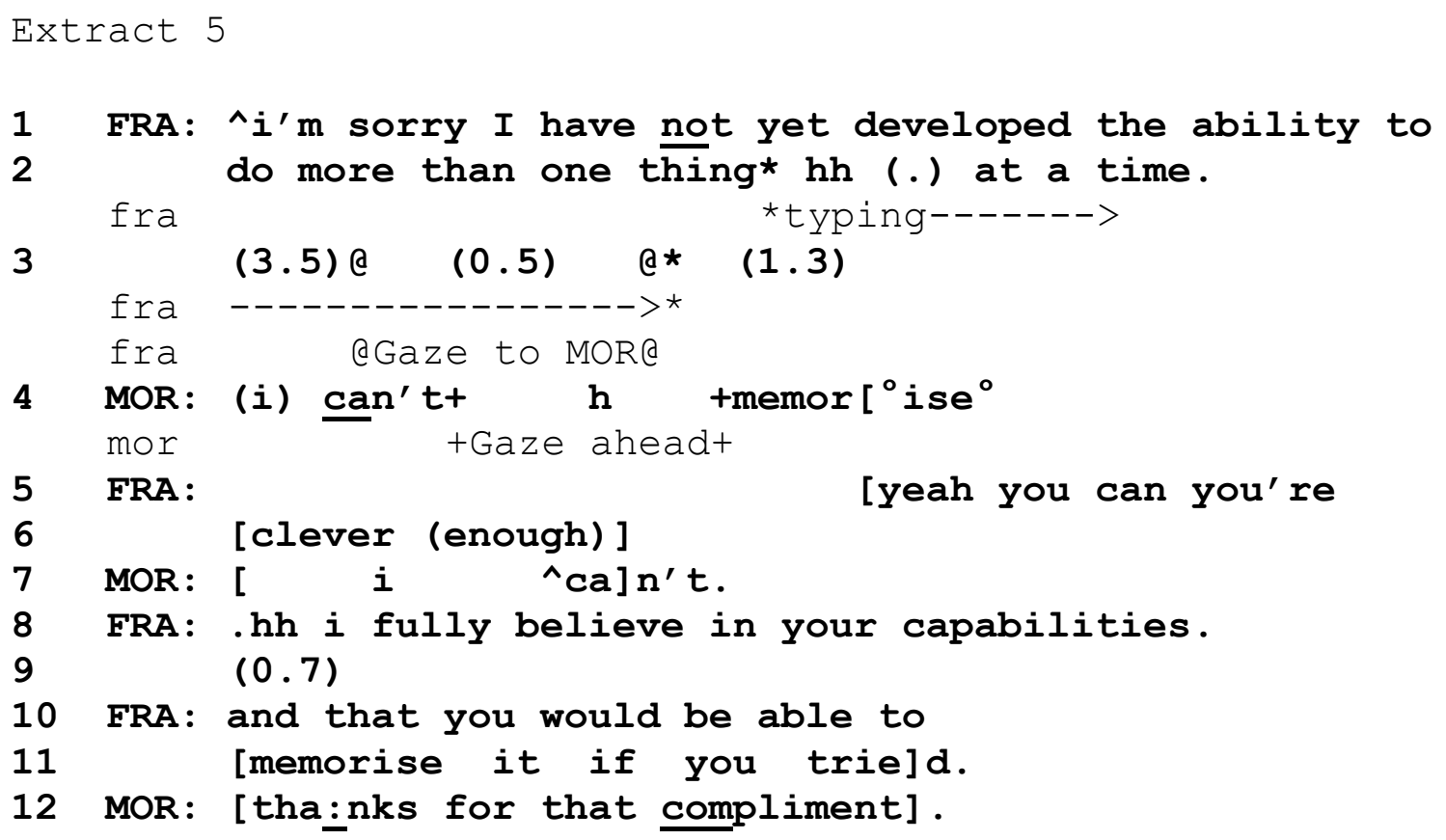

Francesca begins typing in Morgan's password as she finishes explaining to Aidan that she can only do one thing at a time in response to his own request for the password to his device. During her typing, Morgan is gazing to his left, right at the screen. After 3.5 seconds Francesca turns and gazes directly at Morgan. She turned her whole head, quickly to make eye contact with Morgan and she sustains this eye contact until Morgan acts. Kidwell (2005) described this quickly established, extended gaze as a social control practice used by caregivers. In her work, she found that children oriented to these kinds of extended gaze by halting or modifying their behaviour, recognising 'the look' as a comment on their conduct, rather than merely observing their conduct. Here, Francesca successfully utilises 'the look'. Morgan looks away, treating the 'the look' as a directive to change his behaviour (i.e., to stop looking at her typing the password).

Morgan utilises an explanation to address the analysis 'the look' embodies. By providing the explanation that he cannot memorise the password at line 4, Morgan treats the issue that 'the look' is designed to correct as being his ability to remember what he is watching Francesca type. Francesca confirms that that was her concern by countering his claim not to be able to remember with her own, saying that he can remember and is clever enough to do so at lines 5-6. Here, Morgan has shown that he can accurately appreciate the 
analysis of his behaviour embodied in a non-verbal act. He also shows he can design an explanation that attends to that analysis.

\section{Explanations Deployed in Initial Position}

The extracts in this section will demonstrate how explanations can be deployed in their speakers' own initiating moves in order to establish the analytic framework within which their co-occurring or upcoming conduct should be treated by co-participants. Extract 6 shows Morgan and his brother Aidan address one of the video cameras recording them. While Morgan is looking at the camera, Aidan pushes him out of the way to see it for himself. This case demonstrates how Morgan sets up the analytic framework for understanding his conduct (pushing his brother) in order to avoid his conduct being evaluated negatively.

\section{Extract 6}

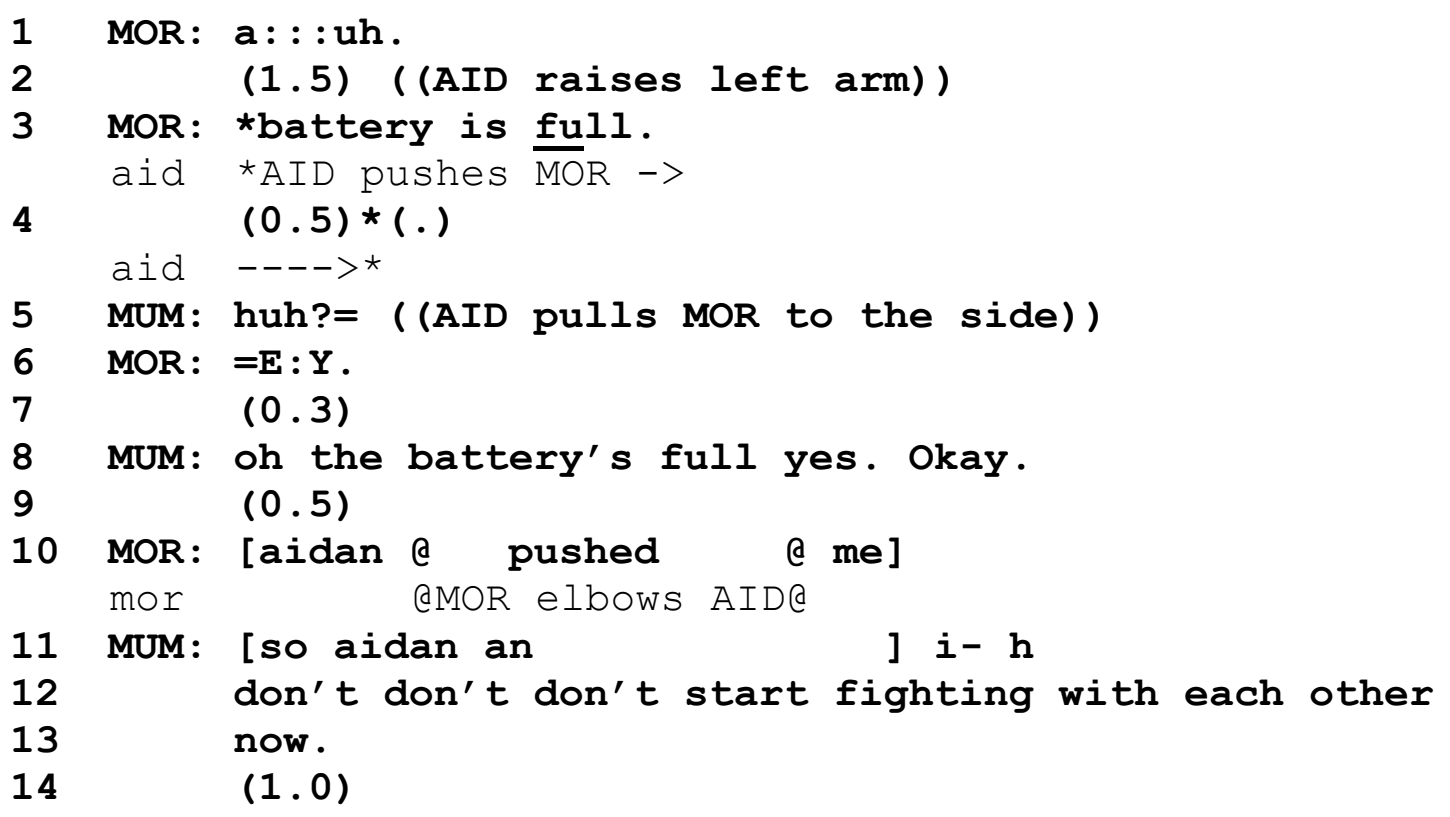

Morgan's explanation warrants his ongoing activity, pre-empting negative evaluation from Mum and/or her call to account. At line 2, Aidan lifts his arm towards Morgan and as Morgan reads the text on the camera screen at line 3, Aidan pushes him out of the way. At line 7 Morgan takes a stance towards Aidan's pushing him, evaluating it negatively with his outcry "E:Y.”. After some silence, where Aidan could have provided his own account for his behaviour, Morgan describes what Aidan did to him, at line 10. At the same time that Morgan begins to say “pushed”, he pushes his elbow into Aidan's back. Morgan's description of what Aidan did to him was built as an account and not merely a description, because he engages in the same behaviour (i.e., pushing) in retaliation as he says it. As such, he constructs his own 
pushing as retaliation, or justice. Without such an embodied response, his talk could have been heard as just a description of Aidan's behaviour. Morgan here attends to how Mum might treat his behaviour, especially in this case as Mum was not watching when Aidan initially pushed Morgan and thus might have treated Morgan's push as the first attack. Morgan demonstrates by his justification that a negative evaluation of his behaviour would have otherwise been warranted and so this is the specific understanding he attends to.

In Extract 7, Mum is looking through one of Aidan's school books after school and recognises she has not seen this book before. Just prior to this, Aidan had read to Mum from one of his other school books. In this case Aidan provides an explanation which sets up the framework within which he proposes Mum understand his conduct (i.e., not reading from this book).

Extract 7

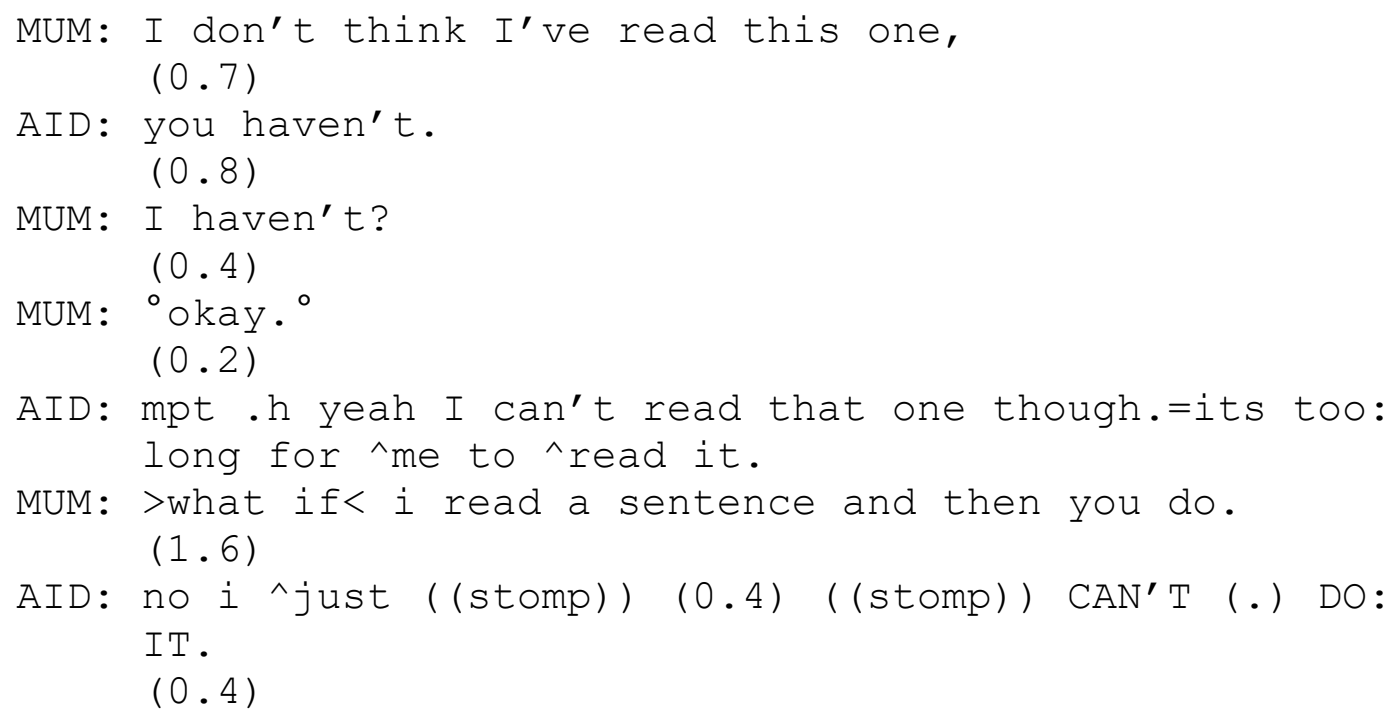

At line 1 Mum notices a piece of writing she claims not to have seen before. After agreeing that she has not seen it at line 3 and Mum closing the sequence at lines 5 and 7 , Aidan, unbidden, says he "can't read that one though." at line 9. Aidan's "though" here sets up the comparison between this moment and the previous one where he non-problematically read to Mum from his book. This shows that this turn is not just an assessment of his own ability. In setting this moment in contrast to the previous, it is an attempt to head off a possible expectation that he read it like he did the previous. Immediately, Aidan appends an explanation for why he cannot read it, saying "its too: long for ${ }^{\wedge} \mathrm{me}$ to ${ }^{\wedge}$ read it." At lines 9 and 10. This explanation serves as both an explanation for why he cannot read it and by extension why Mum should not expect him to read it out loud to her. 
Mum responds to this explanation by proffering a supposedly weakened proposal at line 11; i.e., that she and he could co-read the writing, rather than he alone reading it. This proposal treats Aidan's explanation as purely an inability account and not as shutting down the activity. In turn, Aidan rejects the proposal at line 13 by reissuing the explanation in an affectively upgraded manner by raising the volume of his voice and by physically disengaging with Mum by stomping out of the room.

Aidan's explanation that the text is too long for him to read sets up the framework of understanding Mum should use in designing her next turn. Despite this she pursues exactly what Aidan was attempting to avoid by his explanation.

Extract 8 shows Trevor and his Mum and Dad after having done an Easter holiday egg hunt around their house. Trevor has also received a gift and is about to open it with his parents when Dad asks him about the card that came with it. Trevor's explains why he does not have it with him and this account is built to head off criticism but, like in Extract 7, does not succeed.

\section{Extract 8}

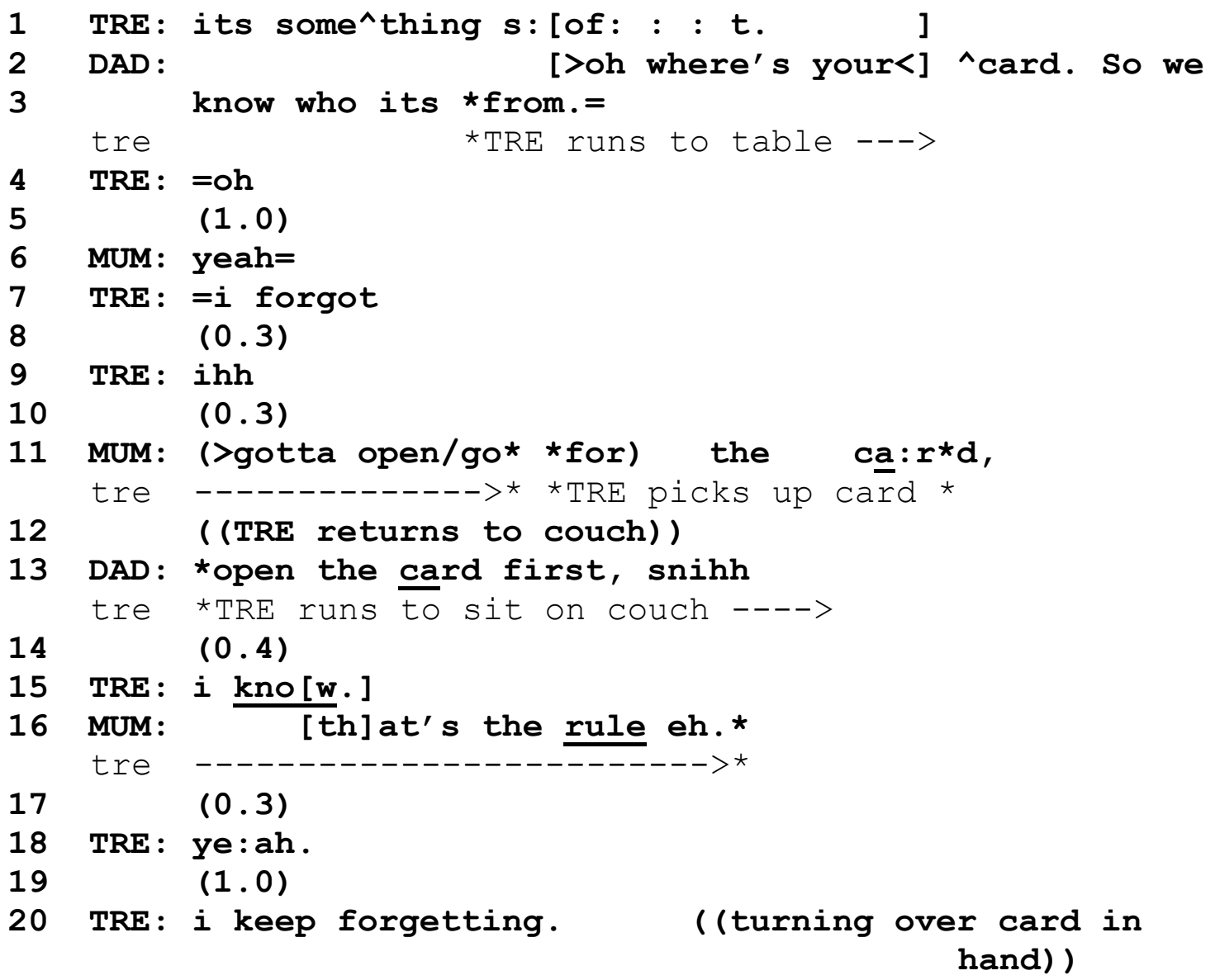


Before sitting down on the couch with the gift, Dad directs Trevor to retrieve the card at line 2. He prefaces his turn with "oh" marking his noticing of the card's absence before asking “where’s your $\wedge^{\wedge}$ card.”. Trevor treats this turn as a directive (rather than a request for information about the location of the card) by turning and returning to the table to acquire it. As he walks to the table, he utters his explanation at line 7; "i forgot". Whereas in Extract 7 Aidan gave his explanation in order to head off Mum's invitation for him to read, Trevor's explanation here is given to pre-empt an accusation of not knowing, or not adhering to, the expectations around gift-opening. By saying that he forgot, Trevor presents himself as less blameworthy for not having kept the card with him as it suggests that had he remembered he would have done it. His explanation pre-empts the presumption that he wilfully ignored the card. This utterance is heard as an explanation because it occurs during the remediation of behaviour that Trevor himself publicly oriented to as accountable.

Like in Extract 7, Trevor's explanation fails to prevent his parent's negative analysis of him as both Mum and Dad treat Trevor as if he does not know the rule of opening cards before presents. Mum and dad both give slightly different variations on an imperative to open the card first at lines 11 and 14 before mum explicitly describes doing so as the rule at line 17. By this time, Trevor is sitting on the couch with his gift and card and, like in Extract 7, he provides an upgraded explanation for his behaviour at line 22. In this version of his explanation, Trevor has not just forgotten this time, he keeps forgetting.

These explanations are designed to set up the framework of understanding that their interlocutors should use (from the perspective of the explainers) in comprehending their behaviour. This makes clear why the children explain themselves again in Extracts 7 and 8; Because their interlocutors make exactly the responsive moves that the explanations were pre-empting. Aidan's Mum still launches a proposal for Aidan to read aloud and Trevor's Mum and Dad treat him as if he were shirking the gift-receipt rules. By doing so, the parents misaligned with the framework of understanding the explanations set up and disregard them. The children's second explanations work to provide another position within which their demonstrably preferable analysis of their behaviour can be affirmed, or at least not disregarded outright. This demonstrates these children's concern with their parents' characterisations of action and how they can recognise when their parents have not accepted their provided analysis. This recognition does not come from a theorising about their possible change in mental states, but through an appreciation of their social actions. 
Why does Morgan not provide another explanation? In Extract 6, Morgan's explanation was given in order to pre-empt Mum's chastising him for pushing Aidan. Mum's response to this is, indeed, to issue a directive not to fight. However, the directive is not singularly addressed to Morgan, but to both children. This directive characterises both children as at fault for pushing each other and are thus not differentially responsible. Morgan escapes any specific scolding and demonstrates by his lack of response that no further work need be done.

\section{Summary}

This chapter addressed the significant cognitive element typically recognised as central to the characterisation of autism; concern for other people's thoughts, feelings and understandings. Autistic people are generally thought to have difficulty in, or a reduced tendency towards, considering other's perspectives. However, these analyses of some autistic children's talk in situ has shown that other's understandings of their behaviour (in the form of what they consider to be appropriate responses to their behaviour) are a salient concern. This is seen in their deployment of accounts.

The children in this data produced sensible accounts for behaviour in response to a first pair part. What this showed is that the children were recognising the first pair part (e.g., a directive) as making some kind of characterisation of the child or taking some kind of stance on expectations that they have of the child's conduct. The children were able to reason out the normative order at work in these contexts by providing accounts when one's behaviour goes against said characterisation or displayed expectation. The children in these cases were able to recognise the initial utterances as launching these kinds of directives or challenges to sense and provide some account that orients to the matters embedded in the first pair parts. In this way, the accounts were designed to manage a first position construction of the child and their behaviour from second position.

The children in the data also used accounts from first position to set the moral framework within which their behaviour should (according to them) be recognised and treated by other co-participants. In these cases, the child claims a higher position of epistemic status regarding their behaviour and its meaning in context by specifying how their behaviour should be treated by co-participants before they engage with it. These accounts oriented to possible ways that others might treat their conduct by dismissing those ways for those the child displays a preference. These kinds of accounts pre-empted possible response to the 
speaker's behaviour. As such, they comprise a kind of reflexivity where the child produces themselves as aware of at least some of the ways they are recognisable by others and presenting themselves as if from another's perspective.

A couple of the children also produced explanations that did not just address local interactional concerns but also explicitly reproduced the common-sense link between mental or internal causes and outward behaviours. While accounts do not necessarily invoke a causal direction from interior to exterior states, deploying an account that does so demonstrates a degree of socialisation into a common cultural understanding of causes of behaviour.

When considered with regard to experimental research and the resulting characterisation of autism that suggests autistic people do not tend to, or struggle to, think about their own or other mental states, it is clear that studies of autistic children's talk provide a much more nuanced picture. These analyses of accounts address the concerns raised regarding Self-oriented social cognition by recognising the practical reasoning about the Self the children do in the course of other activities. For example, providing both responsive and pre-emptive accounts address the Other by responding to and moderating people's characterisations and expectations as well as practically accomplishing a Self as (possibly) seen by other people. As well, accounts that construct the normative mind/behaviour causal link constitute a practical reasoning about one's own experiences and their meanings. Analysing the accomplishment of this social cognition as it is embedded in these children's talk exemplifies the kind of challenge to the narrative that decontextualised and deficitoriented research produce. In the same way, the following chapter will continue to challenge and provide nuance to these narratives of autistic social action by following recent research's explorations into autistic embodiment. 


\title{
Chapter Five: \\ Approach and Avoidance of Affectionate Touch by Autistic Children
}

\author{
"The performance of hugs in the family \\ constitutes and embodies the current states \\ of participants' relationships, indexing \\ how members are attuned with one another."
}

(Goodwin, 2020, p. 34)

Autistic people have reported sensorial experiences that appear different from neurotypical people's experience of senses, specifically touch (Jones, Quigny, Huws, 2003; Grandin, 1992). Autistic people's experience of touch has been studied via report and experimental approaches (Cascio, et al., 2008; Foss-Feig, Heacock, \& Cascio, 2012), and neuroscientific research has been used to explain differences in sensitivity (Kaiser, et al., 2016). This touch sensitivity difference is reported alongside the observation that autistic children engage in affectionate touch less than neurotypical children (Baranek, 1999; Vostanis, et al., 1998; Clifford et al., 2012), with the implication that autistic children's sensory differences impact how much they tend to engage in social touch (Smirni, et al., 2019; Hilton, et al., 2010).

This chapter will analyse episodes of touch between the autistic children and their family members who participated in this study using multimodal conversation analysis. These analyses will demonstrate how touch is not just a sensory experience to these children; touch is also both a resource for action and an environment within which other actions can occur. By analysing how moments of affectionate touch (in the form of embraces or cuddles) are invited, entered into, and/or rejected, I aim to demonstrate how the autistic children analysed make public their priorities for their own courses of action and produce themselves as autonomous. This analysis challenges the commonly presumed strong link between autistic touch sensitivity and physical touch avoidance demonstrated in the literature.

\section{Touch in Social Interaction}

"Touch is a signal in the communication process that, above all other communication channels, most directly and immediately escalates the balance of intimacy" (Thayer, 1986, p. 
8). Interpersonal touch in social interaction serves to build relationships with the self and others. By touching each other and allowing ourselves to be touched we come to a sensual understanding of who we are and who we are to each other. Interpersonal touch is thus the embodied instantiation of relationships between people and is suggested as a primal, initial form of intersubjectivity, called intercorporeality, through which we come to know ourselves and others not just as minds, but as full interacting bodies (Maclaren, 2014).

Psychological research into the social importance of interpersonal touch has looked at the variety of ways that it happens and how people understand the intimacy and meanings of touch. Studies of touch have involved participants writing of their experiences of touch and being touched in private (Willis \& Rinck, 1983), observations of couples in public (Willis \& Briggs, 1992), ratings of the meanings of touch in images (Burgoon, 1991), and experimental studies assessing ability to "decode" emotions from touch alone (Hertenstein, Keltner, App, Bulleit, \& Jaskolka, 2006). Touch in interaction is said to express "more composure, immediacy, receptivity/trust, affection, similarity/depth/equality, and informality" and that different forms of touch can vary on each of these dimensions (Burgoon, 1991, p. 254). The meaning of touch is also thought to vary as a function of who is touching who (Heslin, Nguyen, \& Nguyen, 1983). These studies show that touch is imbued with social meaning.

The social sciences have generally seen an increase in concern with the body in general and a significant site and resource for social action (Thanem, \& Knights, 2019). For example, Foucault $(1984 ; 1991)$ wrote about how the body became a significant site for political power through regulation of how people live and act. Importantly for the purposes of this chapter, this embodied turn was also taken up in studies of language and social interaction. For example, Goffman (1990) studied the way that social actors managed their bodies and manner dramaturgically in face-to-face social interaction. Over the last 40 years, analysts of language and social interaction have been increasingly drawing on elements of the embodied and otherwise material resources available and used in social interaction (Nevile, 2015). The next sections will briefly review the outcome of that increased attention.

\section{Interactional Studies of Social Touch}

Goodwin (2013) described all the different resources people have for designing their actions at any time (i.e., language, gesture, bodily configurations in space, uses of objects et cetera) as semiotic fields which are laminated together in order to produce the local context which participants to an interaction orient to. Touch and embodied configuration are thus to 
be understood by participants and analysts with consideration of their lamination into a contextual configuration with other semiotic fields in order to understand their meaning.

Interactional research has begun to look at the significance of touch as a resource for action by studying when and how touch happens in naturally occurring interaction. This growing area of research has shown touch to be an important resource in the construction of action in a variety of different contexts including in reference to other's or ones on body parts in medical contexts (Nishizaka, 2007; 2011), facilitating or directing people's behaviour in both health-care and child-care/parent-child interactions (Cekaite, 2010; Cekaite, 2016; Marstrand \& Svennevig, 2018), providing comfort and soothing children (Cekaite \& Bergnehr, 2018; Cekaite \& Kvist Holm, 2017; Goodwin, 2017; Kupetz, 2019), and escalating conflict (Whitehead, Bowman, \& Raymond, 2018). That interpersonal touch can have a role in such a variety of different actions and contexts demonstrates Goodwin's (2013) claim of the lamination of semiotic fields. The overall contextual configuration provides context to these different touches and helps us produce touch with recognisably different meanings in situ.

\section{Parent-Child Interaction and Touch}

Much of the systematic social interactional research on touch between adults and children has focused on directives and comforting. A directive is an action launched in order to get someone to do something (Goodwin, 2006; these will be further discussed in Chapter Six). Alongside verbal directives, like requests and imperatives, direction can be accomplished through touch. Cekaite (2010) demonstrated how parents can use a touch from behind to shepherd children towards locations and activities. Positioning themselves behind the child places the adult in an inequal position of power over the child as they have access to the child's body while the child has no access to the parent's for similar control or resistance. Relatedly, parents can use touch to manipulate and modify their children's engagement in different actions or with different aspects of the local interactional ecology (Cekaite, 2016). These different engagements are called participation frameworks (Goodwin, 2000). By constraining the mobility of the child parents can guide their child's attention on a singular participation framework, or by sustaining touch in multi-action contexts parents can manage the multiple participation frameworks they and their children are in (Cekaite, 2016).

Parents also utilise touch in comforting and soothing upset children (Cekaite, \& Kvist Holm, 2017; Cekaite, \& Bergnehr, 2018; Kupetz, 2019). Crying-soothing sequences in which 
children display their upset and adults respond with soothing contact have been studied for the ways involved participants coordinate their bodies in the process of soothing (Cekaite, \& Kvist Holm, 2017). This research has drawn on notions from Adam Kendon (1990) and Goodwin (2000) on participation frameworks. Participation framework refers to the mutual orientation to each other that interlocutors embody and make recognisable through talk and spatial organisation and within which interlocutors conduct their action (Goodwin, 2000). One of the basic embodied participation frameworks is the F-formation, in which participants physically orient themselves such that they are easily, mutually available to each other (for example via eye contact; Kendon, 1990). Cekaite and Kvist Holm (2017) describe how different embodied formations afford and constrict activity and thus promote intimacy between adult and child. These formations range from the head-to-head (or $\mathrm{H}-$ ) formation, the partial embrace, and the face-to-face formation. The H-formation constrains the upset child's engagement with other activities such that they are wholly engaged in the intercorporeal experience, and once a certain level of soothing is achieved, adults can shift their formation into a partial embrace of a face-to-fact one in moves towards shifting from upset to normal engagement in other activities (Cekaite, \& Kvist Holm, 2017). As well as this, affectionate touch is used by adults to prompt children into reciprocal affection (Cekaite, \& Bergnehr, 2018).

Recently, Goodwin (2020) documented some of the ways that hugs between parents and children sequentially unfold. Her work focused on how physical embraces between children occurring in leave-taking environments embodied mutual affective stances towards the parent and child. This affective environment is commonly accomplished via the lamination of different semiotic resources, including "an activity-appropriate lexicon (including terms of endearment), expansive pitch range, and voice quality" on top of the physical embrace (Goodwin, 2020, p. 30). This demonstrates how a hug constitutes a multimodal environment in which the relationship between those engaged is intercorporeally (i.e., through touch, embrace or other entanglement of the body) manifested.

This chapter adds nuance to the discussion of social touch in autistic people's social interaction by analysing the touch as a social phenomenon, as a resource for social action deployed in the moment, rather than as primarily a sensory experience. By analysing when and how the participants engage or disengage with a particular kind of social touch (i.e., an embrace, or cuddle), I aim to demonstrate how this kind of touch is not to be thought of as 
only a sensory experience, but as an interactional environment in which the children analysed can negotiate their own bodily autonomy and prioritise their own action.

\section{Analysis}

The following analyses are organised into two sections. The first section will analyse two cases in which children engage non-problematically in cuddles with their parents. One is a cuddle initiated by the child, the other is a cuddle initiated by the parent. The second section will analyse three cases of problematic cuddles. One case will show a child initially resisting, delaying, and then engaging in a cuddle. The second will show that child resisting before being non-consensually embraced into a cuddle and then ultimately struggling out of the cuddle. The last will show a case of what looks like the child rejecting a cuddle but is actually a case of misalignment between mother's and child's courses of action.

\section{Straightforward Cuddles}

In this section, I will present analyses of two straightforward cuddles. The first case is an example of a cuddle done for its own sake (i.e., as a cuddle) initiated by the child. The second case is an example of a cuddle done in the service of another activity (i.e., playing a riddle game together) initiated by the mother. Both of these cuddles are engaged in nonproblematically by the child. Both cuddles are also sequentially situated between courses of other actions (i.e., they are not interruptive of the child's ongoing course of action). This will be important for understanding some of the problematic cuddles I will analyse later.

This first analysis examines a case of a cuddle initiated by the child. Not only does the child initiate the cuddle with his parent, he shows a sophisticated interactional competence with the resources available to him and Dad (e.g., turn-taking and sequence orientations as well as bodily/gestural resources) for structuring this interactional moment. Prior to this extract, Magnus has voiced his concern that Dad may die soon due to an injury he sustained. Dad has been reassuring him that he is not going to die. Magnus initiates the cuddle after Dad issues the upshot of this reassurance talk. 


\section{Extract 1}

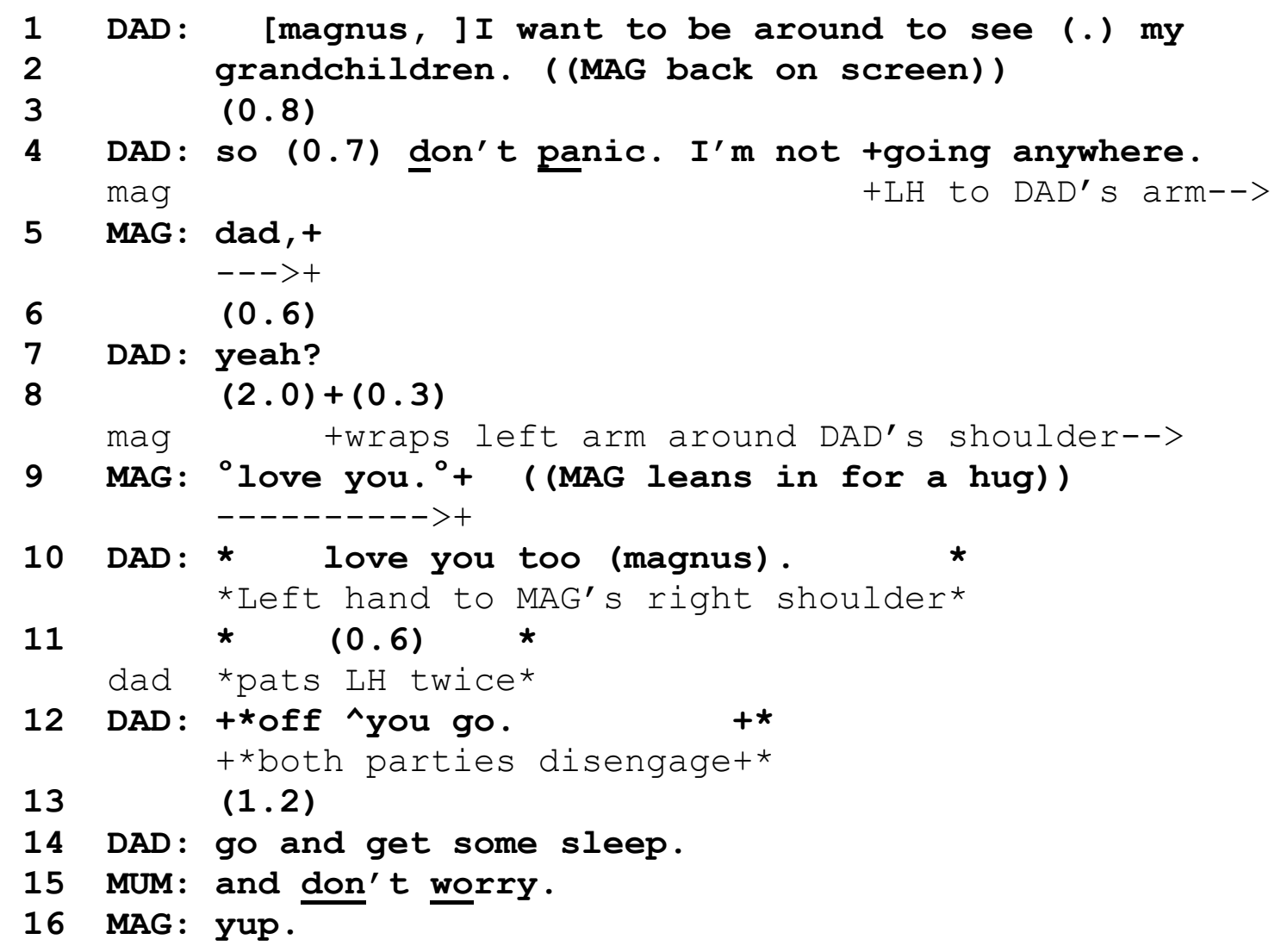

Magnus initiates the cuddle at a point where Dad's completion of not just his current turn, but his current course of action is projectable. At line 1, Dad states he wants to live to see his grandchildren. This is an indication of how long he intends to live as the oldest of his children (Magnus) is 11 years old at this point; grandchildren are temporally distant. This move is not taken up by Magnus at line 3. So-prefacing is a way by which a speaker can mark their upcoming talk as the upshot of previous discussion (Raymond, 2004). It is not until after Dad provides his so-prefaced upshot that Magnus puts his hand to Dad's arm. This movement occurs concurrently with Dad's next turn constructional unit "I'm not going anywhere.". This is a way by which Magnus uses his body to demonstrate his understanding that "so (0.7) don't panic." is an upshot and as such Dad is coming to the end of his current course of action. This would warrant Magnus's transition to a new activity (in this case, cuddling and verbalising affection). However, his touch is not disruptive of Dad's continued talking. Magnus himself does not talk until after Dad completes his current turn constructional unit. How do we know that Magnus was gearing up towards a new course of action? In dyadic interaction, address terms are redundant and are thus marked. Rendle-Short (2007) argues that pre-positioned address terms used by journalists and in political interviews are used to 
structure the interview, managing transition from one topic to the next. In line 5, after Dad has finished speaking, Magnus says "dad,", addressing him. At line 5, Magnus shows he is switching courses of action by re-engaging Dad's recipiency as if it were for the first time. Up to this point Magnus has organised his upcoming cuddle in relation to Dad's talk and his projection of when and how it will end, allowing him to prepare his own new course of action.

The cuddle is designed as a space for affection to occur within. At line 7, Dad responds to Magnus addressing him and at line 8, Magnus wraps his arm around Dad's shoulder and moves in for a hug, concurrently saying "olove you. ${ }^{\circ} . "$ Not only is affection verbalised, the cuddle here is constructed via a head-to-head formation. In soothing sequences, a head-to-head formation is employed as it physically engulfs the upset child's attention, constraining the child's capacity for engagement in any other activity that the embrace (Cekaite, \& Holm Kvist, 2017). This cuddle is not in the context of soothing, however, it still involves the production of intercorporeality (Goodwin, 2017) whereby bodies are entwined with little possibility for distraction from that fact. This embodied configuration contributes to the affectivity and intimacy of the cuddle. Dad reciprocates, wrapping his own arm around Magnus and respondes "love you too (magnus).", ratifying and contributing to this intimate moment.

While Magnus sensitively negotiated the entrance into a cuddle between him and Dad, Dad manages their mutual disengagement from the cuddle and transition into the next activity. He does this using an embodied practice (i.e., patting) that Magnus comprehends. When Dad finishes his turn constructional unit, there is a silence, during which Dad pats Magnus twice. These pats, coming after the reciprocation of affection, coordinate the mutual disengagement from the cuddle. The patting is understood as a signal to end the cuddle due to its placement. Rather than at the beginning of, or during, Dad's reciprocal "love you too" (where it could be understood as part of the affection), the pat comes after he has reciprocated his affection which closes an adjacency pair sequence. There is no specifically selected next speaker or next course of action. As such the pat punctuates the cuddle, providing a space where both parties know it would be appropriate to withdraw. After the 0.6 second silence and the two pats, Magnus and Dad withdraw from the cuddle at the same time. Magnus treats the pats appropriately as non-verbal, touch-based markers of Dad's readiness to disengage from the cuddle. Without speaking or even eye contact, the two were able coordinate their bodies to mutually end the cuddle and transition to saying good night. 
In this second case, I analyse an example of a cuddle initiated by someone other than the autism-diagnosed child; the mother. As well as differing from the previous case in terms of who initiated it, it is also different in terms of the concurrent action. While the previous cuddle was a cuddle in its own right, this is a cuddle that also serves as the enduring corporeal organisation for a longer course of interaction; playing a game together.

\section{Extract 2}

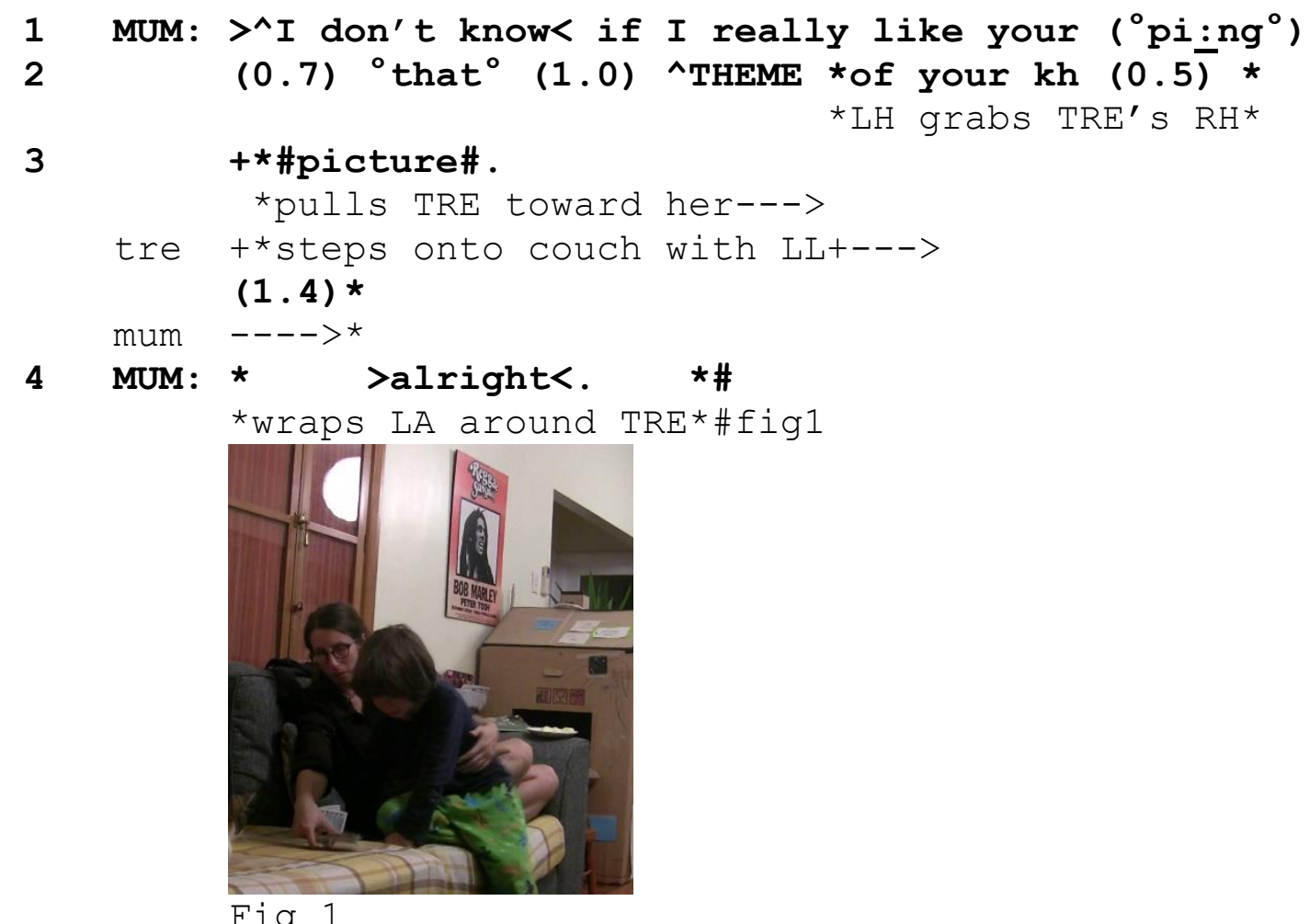

$(0.3)$

5 MUM: *haere mai.

*wraps RA around TRE with cards--->

6 $(0.4)+(1.3) *$

tre $---->+$

mum $---------->$ *

7 MUM: \# Um:

\#fig2

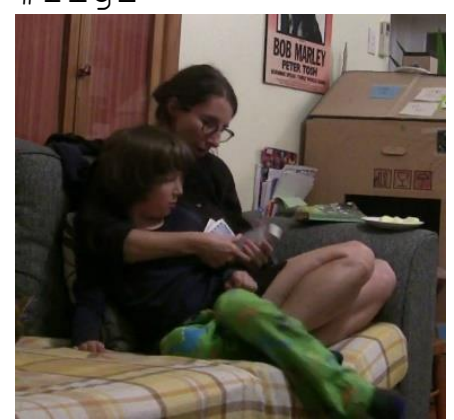

Fig 2

8

$(0.8)$

9 TRE : ( 


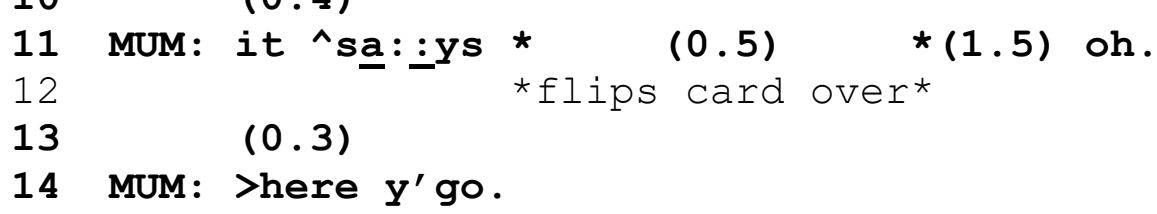

This cuddle is set up at a transition point between activities and directly places Trevor in engagement with the new course of action. Jefferson (1993) discusses the kinds of things that tend to come before topic changes, identifying a speaker's commentary on what has be discussed so far as one of those things. Mum uses her evaluation of the art and story discussed with Trevor as a place to transition to a new activity. Following a 1.4 second silence where Trevor could have addressed Mum's evaluation of his picture and story, Mum utters " $>$ alright $<$ " at line 4 . The silence demonstrates Trevor's orientation to the embodied action at hand and dismissal of the previous topic and Mum's utterance verbally marks the transition to a new course of action, as it is not designed as sequentially related to the prior topic or her prior turn (Maynard, 1980). Ultimately this embrace is initiated in such a way that both participants co-construct it as a transition between courses of action, collaboratively designing the cuddle as something mutual engaged in by them both.

This cuddle having been initiated in this transition between activities is designed to serve as the environment for further action, rather than a cuddle in its own right. Mum initiates the cuddle by grabbing Trevor's arm and directing him onto the couch at line 2. Trevor complies with this, stepping up on to the couch. At line 4 Mum uses her left arm to hold Trevor by the side and guide his body around as he finds a place to sit (seen in fig 1) and at line 5 Mum wraps her right arm around Trevor's shoulder, holding him close to her. Mum has the cards for the riddle game they are about to play in her right hand and so her arm being wrapped around Trevor as it is in figure 2 makes the game mutually orientable by Mum and Trevor. Unlike in Extract 1 where the cuddle was designed as a head-to-head formation between Magnus and Dad, this cuddle is designed such that they can be in contact but still mutually engage in a shared object of activity present in front of both them. Their sitting together on the couch precluded them from engaging in a face-to-face formation as described by Cekaite, \& Kvist Holm (2017), however Mum's arm wrapped around Trevor's shoulder constitutes the same kind of physically close, mutual touch and mutual engagement in a sideto-side formation that the face-to-face would otherwise provide.

These two cases demonstrate the ways that cuddles were positively engaged in between autistic children and their family members. What is seen in these cases is how they 
are situated between other courses of action and are accomplished as mutually engaged-in events even with the initiation of the cuddle is not necessarily mutual. In Extract 1, Magnus initiated the cuddle as a show of concern after an emotional discussion with his parents, and he did so in such a way that showed his understanding of when it would be appropriate to change courses of action in interacting with his Dad. In Extract 2, Mum initiated the cuddle as a space to play a game with Trevor in at a place where a change in course of action was available and appropriate, and Trevor complied with her initiation. These cases represent the cuddles in my data that were entered into non-problematically and describe how they were accomplished. Rather than social touch aversion, these moments demonstrate the in-themoment appropriately managed touch between autistic children and their parents. The next section will focus on some examples of cuddles that were not so straightforward and suggest that this is because they involved some amount of disruption to ongoing courses of action and accompanying misalignment.

\section{Problematic Cuddles}

The extracts presented here demonstrate cases of autistic children in my data avoiding cuddles from their parents, described here as problematic as they constituted sites of trouble to be managed by the child and their interlocutors. While all children engaged in cuddles nonproblematically, not all cuddles were without trouble. The following extracts are presented to demonstrate what those troubles were and how they were managed, providing evidence that not all cuddle resistance by children diagnosed with autism can be described via symptomatic touch sensitivity, but instead based in the child's prioritisation of different courses of activity.

In Extract 3, Aidan is leaving home for school. He has just previously heard the time and from Francesca and recognises he is late. His rushing to go leaves only minimal time for extended farewells, which Francesca chooses to utilise.

Extract 3

1 AID: \#bye francesca.

\#fig1

2 FRA: e^bye babe.=>can I have a cuddle\# before you go.^

mor aputting left arm in bag strap------> 


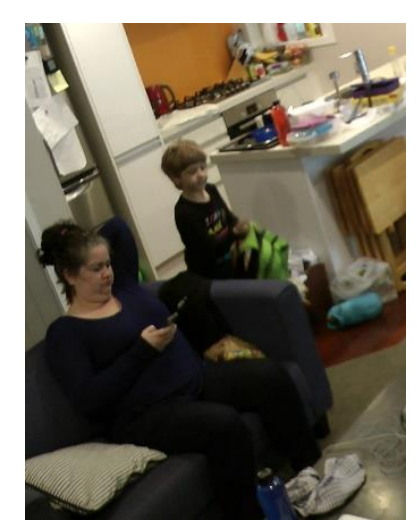

Fig 1

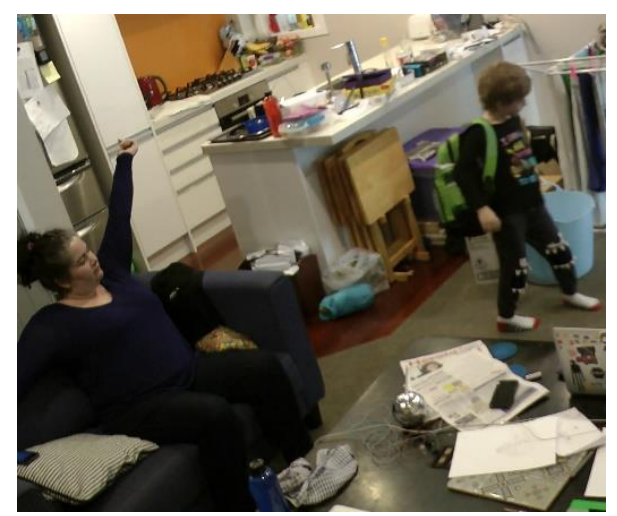

$3 \quad(0.4)$

Fig 2

4 AID : bu-=

5 FRA: ${ }^{\circ} \mathrm{sh}::^{\circ}=$

*lowers LA and shakes 8 times--->

6 AID: i wan[na bring my lego le]vel* crossing=

7 FRA : [hmhm hm hm hm: *]

8 AID : = to $\operatorname{sch}[00: 1$. e ]

*moves arms to head--->

Qquickly faces FRA

9 FRA: *[ah no: . ] $@=$ our lego *\#stays at th [ome]\#.*\#

10 ???:

fra

aid
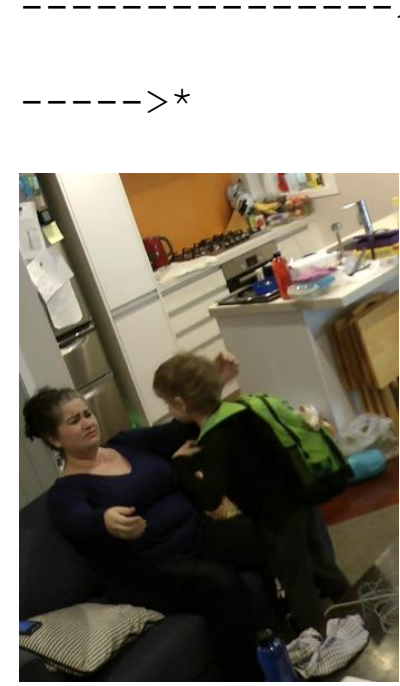

11

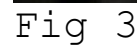

$\star(0.3)+$

aid $----->+$

fra *wraps arms around AID

12 FRA: \#okay\#*\#

\section{[. fh]}

*arms out in front *\#fig3

+ leans down--->

$----->* \#$ fig 4 


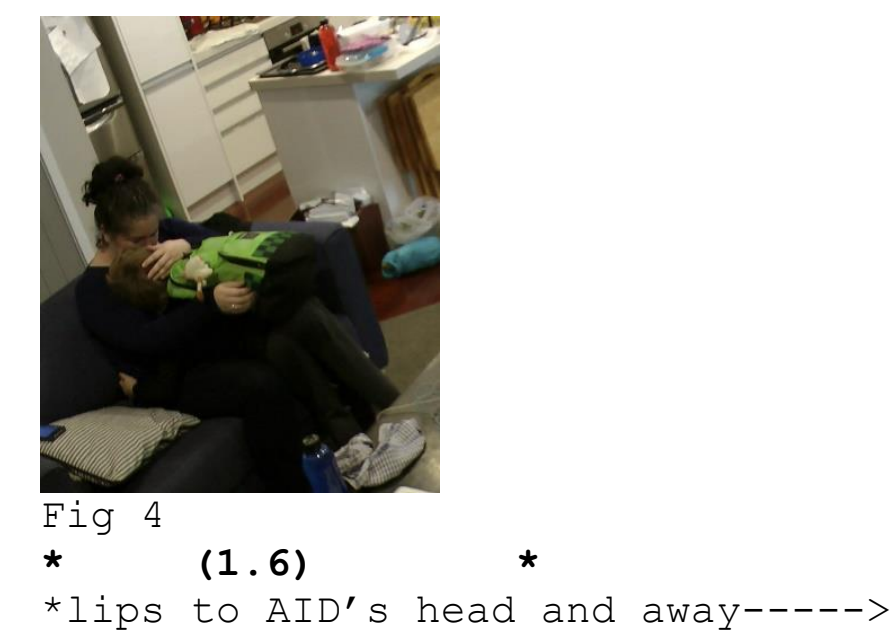

\section{3}

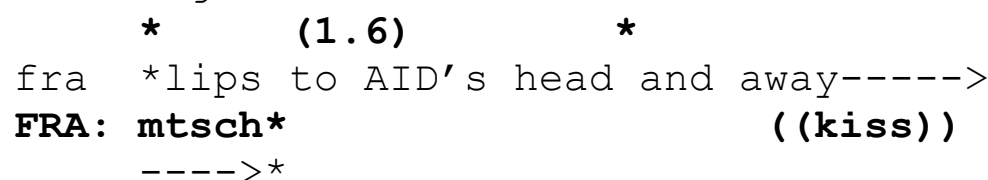

$(0.3)$

16 FRA: plus we don't want it to get lost.

17 mm [mmWAH. ]+

aid +stands up--->

18 AID: [IT won't] $(h)$.

$19 \quad(0.3)+$

aid $---->+$

20 FRA: >have a good< day.

In Extract 3, Francesca requests a cuddle, thus disrupting Aidan's leaving for school. Aidan says goodbye at line 1 as he is walking towards the front door. Francesca reciprocates the goodbye before latching quickly her request for a cuddle at line 2 . This request is made by using a conventionalised request form (i.e., "can I have..."). Conventional request forms have been shown to signal the speakers claimed entitlement to the thing requested (Curl \& Drew, 2008). Thus, Francesca's request claims entitlement to a cuddle before her child leaves for school. In response to this request we can see Aidan immediately stops, turns around, and walks towards Francesca. This embodied compliance is the preferred response to a request for some activity (Kent, 2012), however, he also utters a sound "bu-“" at line 4 which sounds like a cut off "but" on its way to verbalising resistance. While Aidan does what is asked of him, he does not do so without protest.

The request for the cuddle disrupts Aidan's goodbye and reinitialises possibilities for further action between the request and the cuddle itself. At line 5, Francesca wiggles her arms and hums concurrently at line 7 . This demonstrates her receptivity for the upcoming cuddle. However, while Francesca displays her excited expectation, Aidan takes his time before moving into the cuddle as he is still struggling to get his left arm through the strap of his backpack. While he does this, he takes the space available to him to state how he wants to 
bring a Lego toy of his to school at line 6. As Aidan says this, Francesca shuts down her excited anticipation by moving her hands to her head and manipulating her hair; she is no longer immediately anticipating the cuddle as Aidan appears to have moved into a different course of action, at least momentarily. This statement of desire is responded to as an entitled request and Francesca denies it at line 9 and accounts for the denial.

During Francesca's denial of Aidan's request is when the embrace itself occurs. Aidan leans forward and lays his head on Francesca's chest. After wrapping her arms around Aidan, Francesca utilises the interactional space this configuration affords her by giving a further explanation for the account for her denial bounded by two kisses. The first kiss is small and serves as a tactile indication to Aidan that he is the recipient of Francesca's upcoming talk. This required as his embrace configuration does not allow him to see Francesca talking. After this first kiss, she says "plus we don't want it to get lost." at line 16 further explaining why the Lego stays at home. This account is then punctuated by a second, much more exaggerated kiss. This kiss's exaggeration distinguishes it from the first - it is done as a kiss and not as something else, like the previous kiss -- and Aidan treats it as marking the end of the embrace as after this kiss is when he stands up.

Two main features of this case are worth highlighting. Firstly, Aidan verbalises (however minimally) resistance to the cuddle. Despite ultimately engaging in the cuddle, Aidan's resistant "bu-“ interprets the cuddle request as disruptive of his course of action. This resistance is warranted by the fact that he has already made his course of action (i.e., leaving for school) clear. Secondly, after he has abandoned his course of action for giving Francesca a cuddle, he utilises the re-opened interactional space to engage in other activities, delaying the cuddle. This delay is recognised by Francesca, but the cuddle ultimately occurs. This shows that, to Aidan, it was not the cuddle per se that was problematic here, but the fact that it disrupted his ongoing activity. This displayed aversion to the cuddle cannot be understood in terms of anticipation of sensory discomfort or similar, but instead in terms of bodily agency and autonomy in action. Once Trevor was then engaged in the new course of action, the disruptive cuddle was no longer a problem.

This next case demonstrates what appears to be a child resisting a cuddle but is actually a child misunderstanding that a cuddle is what is being proposed. In the minutes leading up to Extract 4, three year old Raphael and seven year old Cecilia have been playing together with some engagement from their Mum. The game has largely consisted of chasing 
and tickling. Of note is that when Mum tickled Raphael and when Raphael tickled Cecilia the tickling has been predominantly focused on the back. This will be significant later in the analysis.

\section{Extract 4}

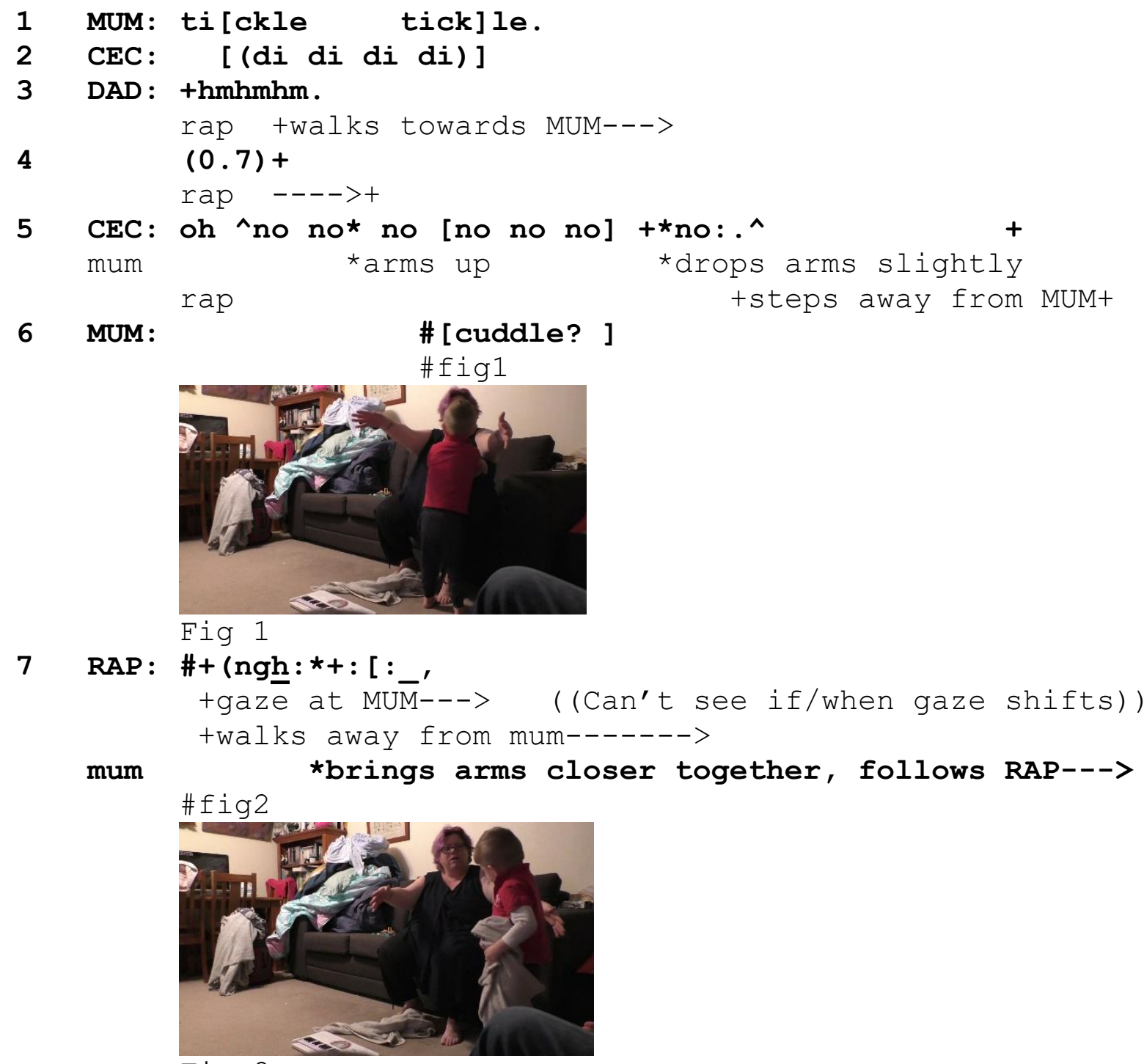

Fig 2

Extract 6 demonstrates how the entrance into affectionate touch is hindered by the local context. Until this point, Mum, Raphael, and Cecilia have been engaged in a chasing and tickling game and this context produces a misalignment in action between Mum and Raphael. At line 1 Mum beckons Raphael with "tickle tickle” and Raphael walks towards Mum at line 3. As he reaches Mum, Cecilia begins shouting " oh ^no no no [no no no] no:.^" at line 5 and this co-occurs with Mum's raising of her hands to receive Raphael and her seemingly re-specifying her activity from tickling (implied by her beckoning at line 1) to now a cuddle at line 6. Cecilia's shouting is much louder than mum's "cuddle?" and it appears to 
have drowned Mum out in Raphael's ears as he seems still to be playing the tickle game with her. After Mum's arms are raised and she requests a cuddle, Raphael turns away at the last minute and begins to walk away from mum, running to the other side of the lounge. As he runs, he is looking behind him at Mum; he is still playing the chasing and tickling game. In this extract, Mum's and Raphael's activities are misaligned. Mum changes course from initiating one activity (tickling) to another (cuddle) but the change is conducted in such a time and place (in the midst of Cecilia's overlapping speech) that Raphael is not party to the change and acts in accordance with the first initiated activity. At this point, the context of play has resulted in a difficulty transitioning into another activity .

Where Extract 6 showed how the local context of play disrupted a cuddle in the making, Extract 7 shows how Mum and Raphael are able to manage themselves and ultimately achieve their affectionate contact (despite complications).

\section{Extract 5}

1 MUM: *^come,

*raises hands,

2

*(0.4) *(0.5)

mum *flicks fingers towards herself*

3 RAP: *+(^I $\neq w i l l)($ ),

( (beckoning))

+steps towards MUM---->

mum *drops hands

mum $\quad \neq r a i s e s$ hands to receive RAP

4

$$
(0.4)+\#+(0.2)+
$$

rap ---->+\#fig3 tbends overt

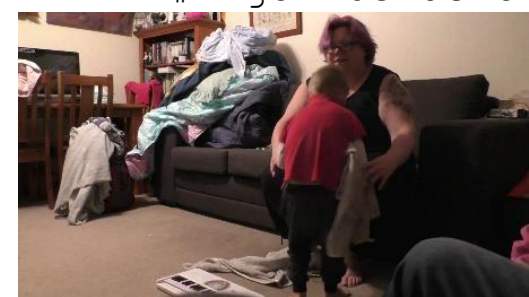

5

$$
\text { Fig } 3
$$

MUM: $* a[:+: w$.

* m: : tchmw\#+ah.*

*hands to RAP's side \#fig4

thands up to RAP's head and away

*lips to head *

6 RAP : [hmm.]

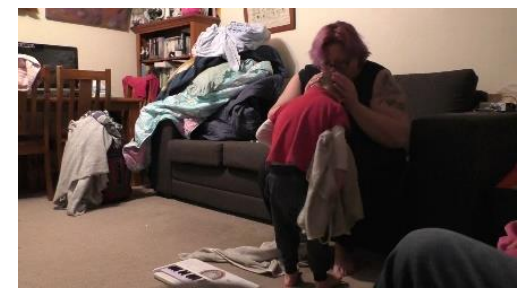

Fig 4 
After Raphael has been playfully avoiding Mum on the other side of the room, Mum calls him over at line 1 with "^come," and at the same time raising her arms and beckoning Raphael with her hands. At line 3 Raphael speaks. Much of Raphael's speech is rather highpitched and unclear. It sounds like he says “^I will” verbally acquiescing, consistent with his embodied response of walking towards Mum. As Raphael walks towards her, Mum raises her arms again before dropping them towards Raphael's side. Ultimately, Mum holds Raphael's head and gives him a kiss. While this is an achievement of affectionate contact, it is not the cuddle Mum initially seemed to be seeking. Recall from earlier that Raphael does not seem to have heard that Mum was after a cuddle as her switch from tickles to cuddles was talked over by Cecilia. As Raphael comes to Mum, he bends over which makes his back easily reachable by Mum. Mum previously had been tickling his back in their ticklingchasing game. Raphael presents his back to Mum in order that she resume tickling like she did in their game earlier because as far as he knows, they are both still playing the game and Mum was still teasing him with tickle threats. Mum's dropping and sudden raising of her arms to holding Raphael's head and giving him a kiss are in-the-moment management of the affordances of Raphael's bodily position as presented to her.

This case shows how two parties to affectionate touch in interaction can be misaligned in their action on the basis of their previous actions and broader activity context and address that misalignment on the go so that an approximation of the originally intended action can be accomplished. Here, Mum and Raphael are working under different presumptions of what kind of engagement is on offer based on each other's understanding of the play context they are in (where Raphael perpetuates play after Mum has concluded it) and yet are still able to coordinate such that affection touch is achieved.

The next extracts show another cuddle engaged in by Francesca and Aidan. Immediately prior to these extracts, Aidan has expressed frustration with his computer. Francesca has asked to know what is wrong so that she can try and help, and Aidan has rejected her requests instead asking for an apple. Francesca has said that him having an apple is contingent on him coming to her first (ostensibly for the upcoming cuddle and discussion). After that, Aidan stands up and walks towards the kitchen. This is when Extract 6 begins. 
Extract 6

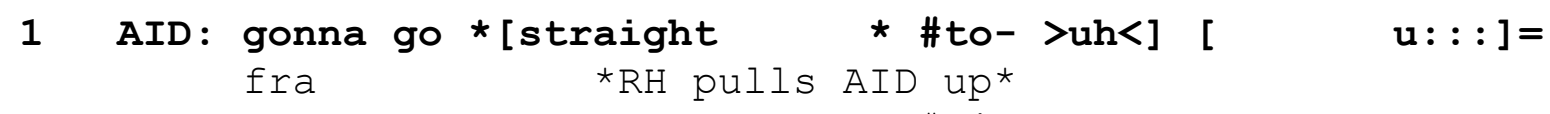

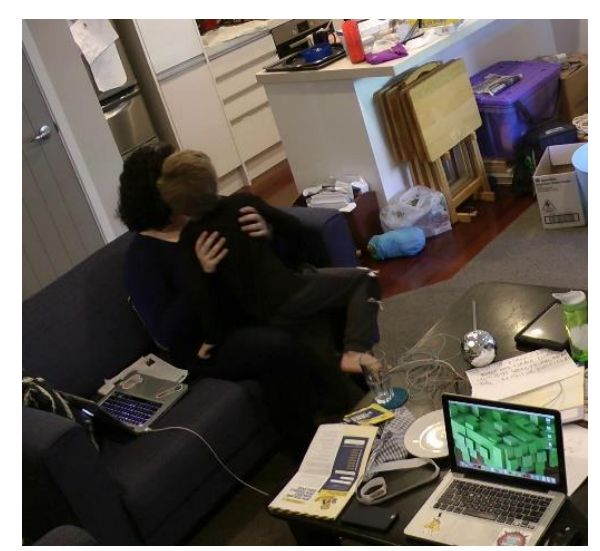

\#fig1

2 FRA:

Fig 1

3 AID: =>hihihihi< hi hi=

4 FRA: >nonono<\# no no [no no no no no: :]

\#fig2

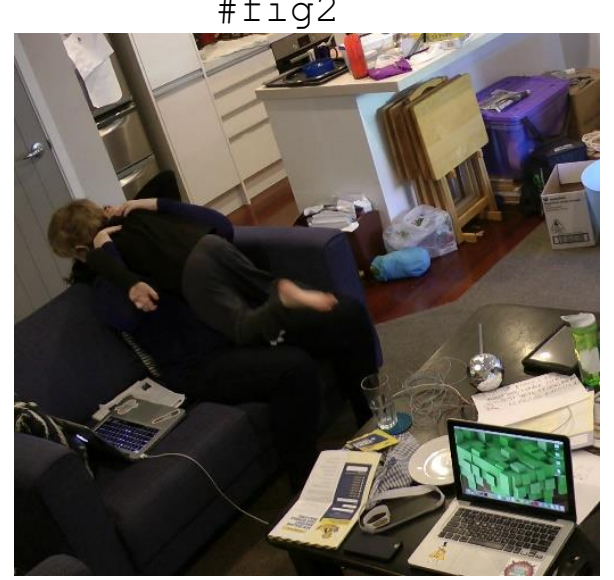

Fig 2

5 AID:

6 FRA: $=[$ don't be happy

[u:: : >hihihi< ] >hihihi< hyu:ck=

7 AID: [.h ha: ha >haha< ha]. H >hihihi<.H=

8 FRA : =* don't be ha*[ppy. *stop * lau]ghing, =

*wraps LA around AID* *wraps LA*

[no no no no]

9 AID : [ $\mathrm{u}:: \mathrm{h}$ ]

10 FRA: =\#[no:. you were busy feeling miserable.] \#fig3 


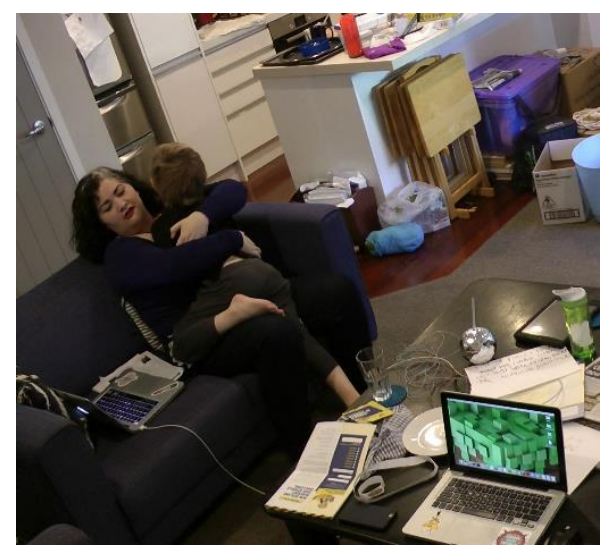

$$
\text { Fig } 3
$$

11 AID: [u>hihi< >hihihi< >hihi< > HAHAHA< ] HA HA HA=

12 FRA: =*[back to being* *miser] a+ble. +

* RA drops * *wraps LA to RShoulder--->

13 AID : [.H a: : : h +wraps RA+

$14(0.4)$ *

fra $--->*$

15 AID: .hh ^u: :.

$16 \quad(0.5)$

This part of the cuddle (Extract 6) is oriented towards Aidan's current emotional state and remedying it, specifically by eliciting displays of positive affect from Aidan. At line 1, Aidan verbalises that he is rejecting Francesca's demand that he come to her and is, in fact, on his way to the kitchen for the apple he asked for. Despite this rejection of close contact, Francesca scoops him up as he walks past. Francesca brings Aidan up into a head-to-head formation (Cekaite, \& Kvist Holm, 2017). Francesca is thus in control of a participation framework between her and Aidan in which Aidan has little choice but to be recipient to Francesca's action. Francesca's actions consist of tickling and talking. The tickling serves to elicit positive affective displays from Aidan, which successfully occurs in lines, 3, 5, 7, and 13 where Aidan is laughing. Francesca's talk seems, on the surface, to be inapposite. At lines 2 and 4 she produces repeated "no"s, rejecting and negatively assessing Aidan's laughter, and at lines 6 and 8 she directs Aidan to stop laughter and stop being happy. Considering Goodwin's (2013) notion of lamination, Francesca's overall action is best understood with reference to all semiotic resources she employs. By considering her talk laminated by her tickling, Francesca's directives and rejections of Aidan's positivity are recognisable as teasing and playful. Exactly their inappositeness is what makes them sound like playful teasing rather than genuine directives. During this, Aidan, despite having resisted Francesca's attempts to help with his frustration, is (at this point) fulling engaged in the ongoing haptic experience. Though this is not to last. 
Extract 7 follows immediately for Extract 6. In this extract, the tickles have ended and Aidan has settled into Francesca's embrace with a face-to-face configuration, prefiguring the discussion they are about to have which raises trouble for Aidan and his continuing within the embrace.

\section{Extract 7}

1

FRA :
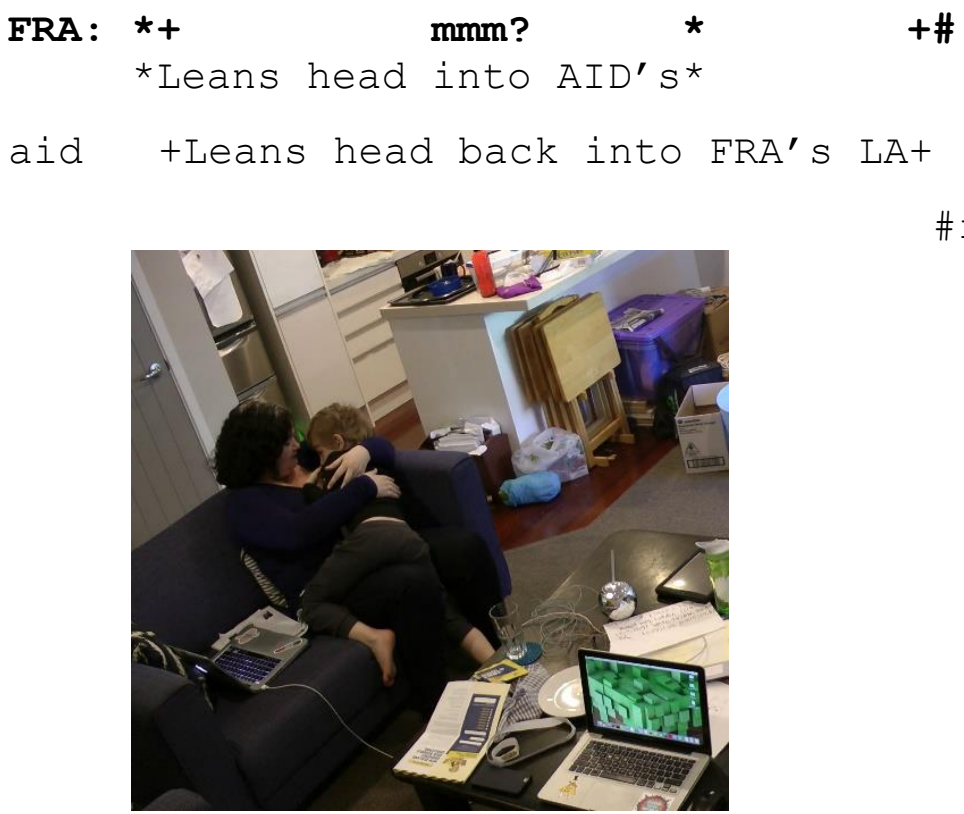

$$
\text { Fig } 4
$$

2

$$
\text { fra }{ }^{*} \text { RH rubs back } 3 \text { times * }
$$

\section{\#fig 4}

aid tgrasps FRA's necklace+

3 FRA: cuddles alwa [ys make you feel better].

4 AID:

AID: [+ungh ungh ungh ungh + ) interrup] te: [d.]

RA :

\section{[1}

[ I ] think

you need to (1.1) *start remembering when you feel * removes AID's LH from necklace-->

\section{Frustrated* that- (1.3) cuddles}

[always help you. feel a bit better.]

fra $+\mathrm{RH}$ rubs back 4 timest ] NO no tickle:s.

11 FRA: and ti[ckles, ]

12 AID: [its ti]ck*le::[s.

* Lifts head---->

13 FRA : [so when +you: $*$
*are on your computert and somethings not going the

aid [so when +you:*
*are on your computert and somethings not going the fra

aid *returns head to FRA's arm-----> way you wantt it to go and you go .h *nguhhh* and you 


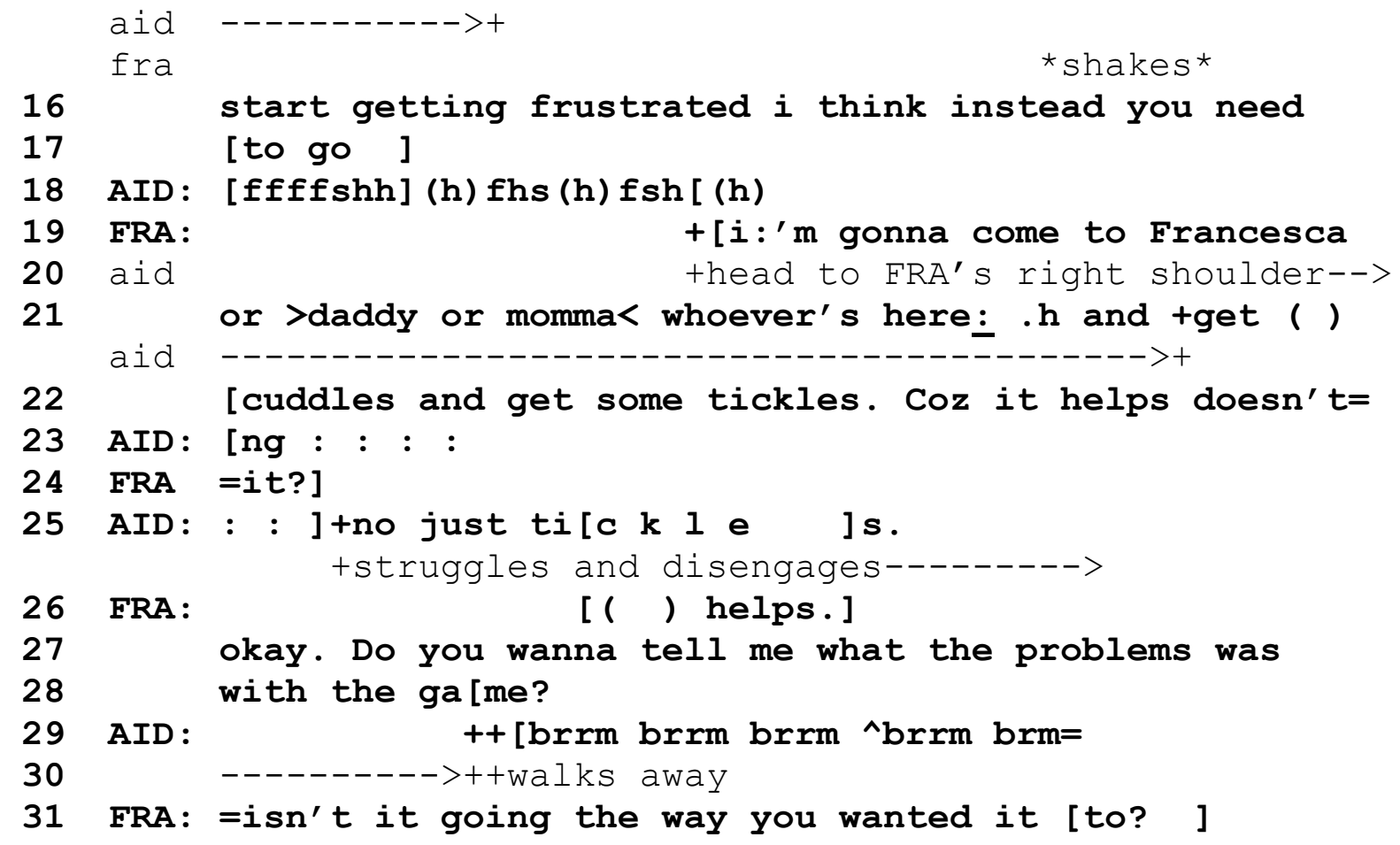

Here, Francesca alters course, turning the cuddle from one primarily oriented to modulating Aidan's affective state to a locus of socialisation into emotional regulation, teaching Aidan how he could manage future emotional incidents. Where Francesca is primarily oriented towards teaching emotion regulation, Aidan shows his concern with how this whole event is to be understood. The tickle game winds down as Aidan rests his head in the crook of Francesca's elbow at line 1. This shift in position brings the two into a face-toface position. This transition to face-to-face position marks Aidan's re-entrance into the role of possible speaker (Cekaite, \& Kvist Holm, 2017), and presages Francesca's expectation of talk from him. At line 3, Francesca assesses cuddles' ability to make Aidan happy. At line 5, Francesca provides the advice that Aidan needs to remember that cuddles make him feel better when he is frustrated. As soon as she voices "cuddles" at line 7, Aidan comes in rejecting the formulation of cuddles as the thing that works and specifying that it is, in fact, tickles (and only tickles) that works at lines 10 and 12.

Francesca provides more advice to Aidan about what to do when frustrated and continues to formulate the significant helping component as cuddles. This continuing rejecting of Aidan's formulation (that it is just tickles that works), prompts Aidan to ignore her advice and repeat his correction while struggling to withdraw from the cuddle. At lines 13-25, Francesca describes a hypothetical future scenario like what happened earlier (where Aidan is getting frustrated by his computer not cooperating) and suggests in such a scenario Aidan should go to someone and get "cuddles and some tickles" (line 21). She finishes this 
extended turn by attempting to elicit Aidan's agreement that it (ostensibly getting cuddles and tickles) helps. Instead of agreeing, Aidan overlaps at the end of her turn to say "no just tickles." at line 24. At the same time, he begins talking, Aidan, struggles free of Francesca. While Aidan is withdrawing from the cuddle, Francesca returns to her initial questioning regarding what was going wrong in the first place, but this is ignored by Aidan and she does not pursue a response. For Aidan, the cuddle was the environment within which he was not being listened to. While Aidan did not initially display enthusiasm for the cuddle (in fact, explicitly moved to avoid it), he did come to settle into Francesca's arms. Her continuing to talk about (and mischaracterise) Aidan's frustration was the triggering event that Aidan responded to by leaving the cuddle. This suggests that it was not anything about the cuddle or touch per se that necessitated his response; it was the course of action being pursued within the cuddle that was problematic.

The last case I will show demonstrates a cuddle between siblings, both of whom have received autism diagnoses, and one of whom is minimally verbal. While there is some initial misalignment, this cuddle is ultimately produced with mutual engagement and it leads into a period of extended touch as the children play together. Up until the point transcribed below, the two children have been playing in the same room but independently of each other. Cecilia is playing with a dollhouse when Raphael approaches her for a cuddle.

\section{Extract 8}

\section{CEC: +\# ((CEC speaking quietly to toys))\#+}

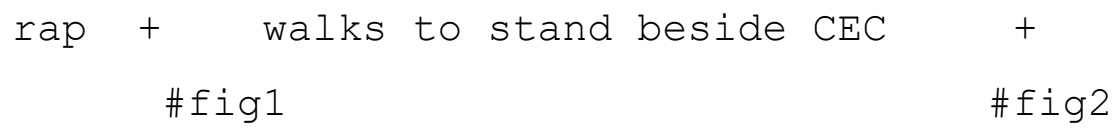

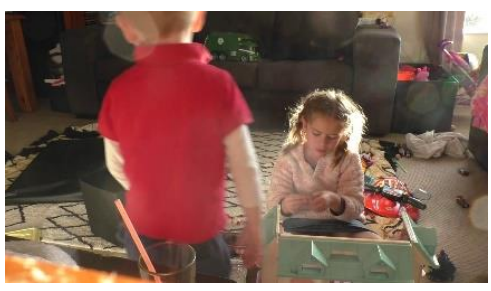

Fig1

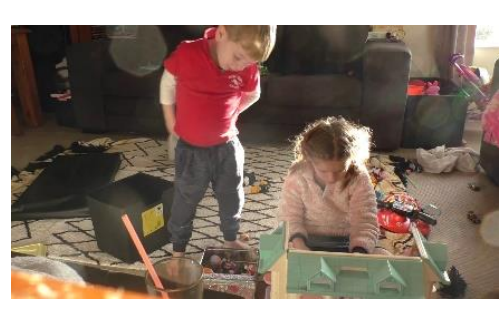

Fig 2

$2 \operatorname{RAP}: \wedge \wedge($ tay) ,^^

3

$(0.6)$

4

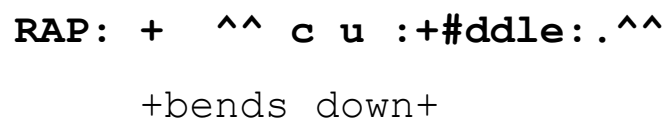




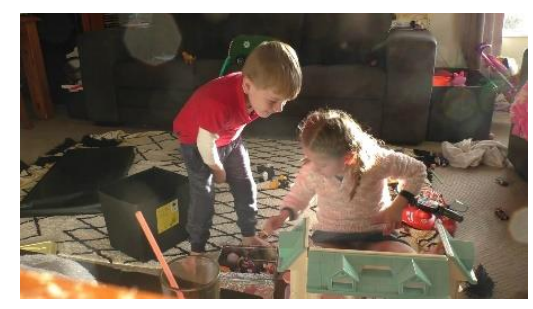

Fig3

In this extract, there is initial misalignment between the two siblings. While Cecilia is engaged playing with her toys, Raphael approaches her at line 1 before bending down to say “^^ c u:ddle:.^^" at line 4. It is unclear at this point whether this was designed as an announcement of Raphael's interest in a cuddle or request, or as an announcement that he was about to engage Cecilia in a cuddle, but what is clear is that he does not immediately engage in the cuddle. This suggests that whatever his turn was exactly designed as, it was at least a first pair part making a response from Cecilia relevant. This is where the initial misalignment occurs as Cecilia does not respond. The fact that Cecilia gives no response, rather than a negative evaluation of Raphael's request/announcement suggests that she is not resisting, she is just engaged in another course of action and prioritising attention on her play.

Extract 9

1

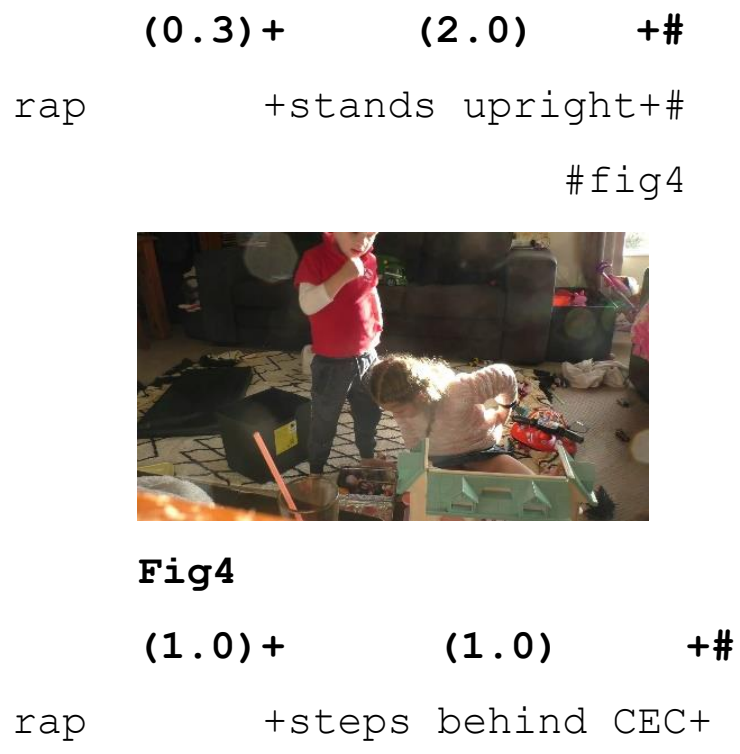

\# fig 5

Fig

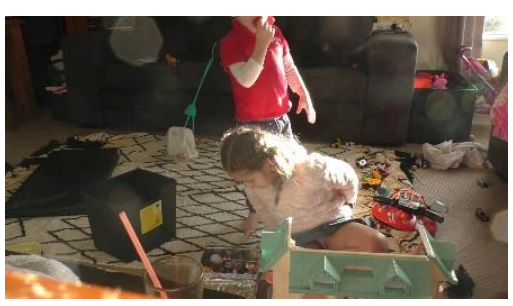




\section{Fig5}

\section{3}

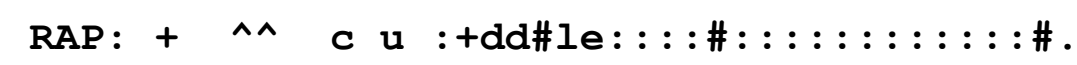

tarms raised+

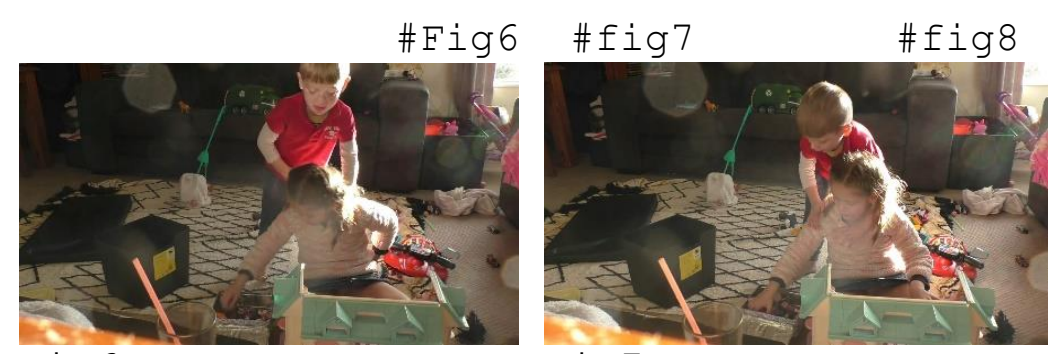

Fig 6

Fig7

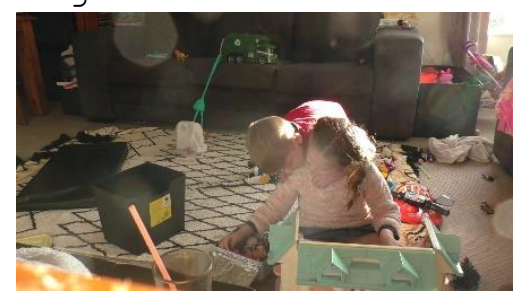

Fig 8

4 CEC : aww* ^ ng : . *\#

${ }^{*} \mathrm{RH}$ to $\mathrm{RAP}^{\prime} \mathrm{s}$ head*

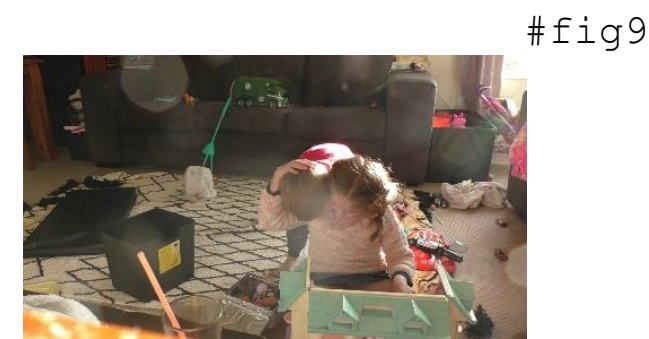

$$
\text { Fig } 9
$$

$$
5 \quad(0.3)
$$

6 RAP: ^^cuddle.^^

7 CEC : aww $(*)$ *

${ }^{*} \mathrm{RH}$ to toybox*

8 RAP: $=+(\mathrm{u}: \mathrm{m}, \mathrm{)}+$

$$
+ \text { stands upt }
$$

$9 \quad(0.3)$

10 CEC: d'you *wanna play with me?

11

*Tilts head back, follows RAP

$$
(0.3) *(0.2)
$$

12 RAP: ^^o:kay,^^

13 CEC: okay come here_( (CEC helps RAP to sit on her lap))

Extract 9 shows how Raphael manages the non-response from his sister after his requesting/announcing a cuddle. After two and half seconds of delay, Raphael announces a cuddle again at line 3. This announcement proceeds from him standing up after his initial request/announcement and stepping behind Cecilia. Once in position behind her, Raphael speaks “^^cu:ddle::::::::::::::.:.^^". Stribling, Rae, and Dickerson (2007) describe how a child 
diagnosed with autism is able to use resources like repeats in contexts where it is not clear that their interlocutor heard them. Raphael's turn is a repeat produced with a sound extension, possibly attending to this as a pursuit or a second issuing, oriented to the possibility that Cecilia missed it. As he produces this turn, Raphael raises his arms before bringing them down around Cecilia's shoulders. In this way he initiates the cuddle on line 3 (figures 6, 7, and 8).

Despite being engaged in a different activity and not taking up the cuddle as initially announced/offered, Cecilia accepts the cuddle and actively engages in it too. At line 4 of Extract 9, Cecilia wraps her right arm around Raphael's head, holding him tight to her shoulder (figure 9). This cuddle resembles a head-to-head formation (Cekaite, Kvist Holm, 2017) in that the two have pressed their heads close together, however it is a fundamentally different organisation employed here. Rather than each in front of the other, Raphael is standing behind Cecilia who is herself facing away from him. By coming at the cuddle this way, Raphael, as the one who initiated the cuddle, was able to embrace his sister in a way that did not impact, or reduced the impact of, her ability to continue doing what she was doing. In this way, Cecilia, could engage with the cuddle, as she does, or continue using both arms to play with her toys and Raphael could still get his cuddle. Once Cecilia returns her right hand to the toys, Raphael stands up and at line 10 Cecilia asks whether Raphael wants to play with her. In this way, Cecilia does not just participate in the cuddle, but uses it as her own springboard to initiate extended affectionate touch and play activity.

The way this cuddle unfolds demonstrates that despite Raphael not talking much and both children being diagnosed with autism, they are both able to competently negotiate a cuddle in these difficult circumstances. This case shows how, even though the cuddle was initiated while Cecilia was engaged in some other action (which, as we've seen above can be a site of cuddle refusal), the cuddle was managed in such a way that it could occur while not being fundamentally disruptive to the course of action Cecilia was engaged in. As well, this case shows how Raphael oriented to the cuddle as naturally implicating someone else in his course of action. He did not just leap right into the cuddle with his sister but indicated that a cuddle was desired and incoming, providing Cecilia with an opportunity to engage willingly or resist. While, ultimately, Cecilia did not respond and Raphael initiated the cuddle anyway, the fact that he did it twice suggests he considered it relevant and important to rather than presume a cuddle would be welcome from the start. 
All of the cases in this section demonstrated some of the ways attempts by parents or sibling to engage in affectionate touch with their autistic family members might unfold in a less-than-straight-forward way. I have shown that looking at the local interactional context of an attempt to cuddle can aid in understanding why the cuddle might have been negatively evaluated (Extract 3), withheld (Extracts 4 and 5), or abandoned (Extracts 6 and 7). As well as these, I have shown how what starts off problematically can be mutually approved in the offing (Extract 8 and 9).

\section{Summary}

This chapter analysed the engagement in embraces by autistic children and their parents/family members. Because literature on touch and autism takes for granted that autistic people avoid interpersonal touch for sensory reasons, cases of interpersonal touch (or where interpersonal touch was invited and negotiated, whether or not it actually occurred) stood out in viewing the data. Analysing these cases demonstrated a technical skill in embodied coordination possessed by both children and family members, both in terms of entering into, exiting out of, and avoiding cuddles. This practical, embodied competence was exemplified most simply in the first two cases analysed. One of these cuddles was initiated by the child and the other by a parent. For example, Extract 1 showed how Magnus was sensitive to the sequential position in which a change in course of action would be appropriate (demonstrating again some of the Other-oriented concern discussed in the previous chapter by not interrupting Dad), and sensitive to the possibly embodied, tactile cues that can be used to mark the end of the cuddle for a smooth, mutual disengagement.

When it came to cases where interpersonal touch was on the table and resisted, avoided, or abandoned by the child, a lot of small yet significant local interactional contingencies impacted how it unfolded. Primarily, all of these cuddles involved some degree of misalignment in activity between the child and the family member. These misalignments are the result of the children, as potential cuddle recipients, prioritising some other course of action they were already engaged in. Some of these cases involved protests snd heightened negative affect against the disruption the cuddle produced, while others were more peacefully remedied. Fundamentally, these cuddles were not treated as problematic by the children due to the sensory nature of the touch involved, but due to the significance of the action at that sequential position within other courses of action. 
These analyses of interpersonal touch between autistic children and their family members demonstrates two significant things about common conceptions of both autism and touch. The first is that, while research literature has tended to take for granted that social touch aversion in autistic people is related to the sensory experience of touch, that is not necessarily so. All people will experience negotiating being touched at some point and not just because of what that touch feels like but because of when it happens and what it means. By negotiating when and how they get touched, the children in this data are taking a stance on their autonomy as active social agents. Seeing the problematic (to neurotypical people) behaviour of psychiatrically diagnosed people as a manifestation of some psychiatric symptom removes the agency of the diagnosed. Seeing this autonomy in action repositions autistic people as actively engaged, functional, and agentic.

The second thing is that, while touch is generally considered as a sensory phenomenon in psychological literature, this is actually a limited perspective. Touch does entail a sensory experience but also much more. Cuddles, for example, are not just times when two people's arms wrap around and feel each other; they are environments in themselves in which other activities can be accomplished. In this data, cuddles were sites for sharing affection, for providing advice on emotional matters, for play (and initiating play). Focusing on the sensorial or tactile experience of touch ignores the fact that touch is bound up within courses of action, enabling and constraining certain kinds of actions and relations.

This chapter addressed an issue associated with embodiment and autism. The next chapter will draw ideas from this and the previous chapters so see how issues within the cognitive and sensorial domains are managed within the course of longer, ongoing interactional sequence. It will demonstrate how issues of embodiment and touch, emotion, knowledge, and relationships come to be embedded into the unfolding of another activity; the negotiation of an activity contract in an extended directive sequence. 


\title{
Chapter Six: \\ Negotiating an Activity Contract Between an Autistic Child and their Parent
}

\author{
"Family members engage in considerable negotiations \\ with respect to rights and responsibilities regarding \\ the timing and progress of activity in the household. \\ Parents serve as time managers $[\ldots]$ and children \\ constantly bargain about parental time regulations $[\ldots]$ ”
}

(Goodwin, \& Cekaite, 2018, p. 83)

Parents report that parenting an autistic child is difficult (Meirsschaut, Roeyers, \& Warreyn, 2010; Ooi, Ong, Jacob, \& Khan, 2016). Getting an autistic child to do something is a demanding task. A review of qualitative studies on parenting autistic children (O'Nions, Happé, Evers, Boonen, \& Noens, 2018) reported that managing non-compliance and distress are two of the nine major reported parenting concerns. Parents report repeatedly issuing directives to their autistic children and using various other strategies to reduce the chances of non-compliance, like developing routines, breaking activities into smaller tasks, behavioural reward strategies, giving choices, and tricking/coercing (Larson, 2006; Neely-Barnes, Hall, Roberts, \& Graff, 2011; Johnson, Bekhet, Robinson, \& Rodriguez, 2014; O’Nions et al., 2018). As well, research shows autistic children's' pragmatic difficulties can commonly result in non-responses to parents' initiating turns (Loveland, Landry, Hughes, Hall, \& McEvoy, 1988), and autistic children are less likely to respond to parents' bids for joint attention (including those in the form of directives) to objects (Adamson, McArthur, Markov, Dunbar, \& Bakeman, 2001).

This chapter presents an analysis of a single, extended interactional project. In this case, Mum is attempting to get to Trevor to use the bathroom before bed. This analysis demonstrates some of the variety in activities that that can be engaged in in the process of securing compliance with a parental directive. These different activities involve negotiation between Mum and Trevor about who they are to each other and their responsibilities to each other while also serving as sites where management or affect and knowledge is relevant. This analysis shows how directing an autistic child to act is more than just a case of telling 
children what to do; it is also a site for the mutual construction of the participant's selves and their relationships.

\section{Deontics in Interaction}

The issue of getting children to do tasks is one of deontics, a term which refers to the social capacity to determine action (Stevanovic, 2018). The deontic domain in interaction concerns the locally negotiated rights to direct one's own or another's actions. In social interaction, people negotiate their deontic stances and statuses. Deontic stance concerns the publicly available claims to the rights to direct some action, while deontic status refers to the rights to direct some action that a person is considered to have by others, regardless of their claims (Stevanovic, 2018). When parents ask (or tell) children to do something, they publicly take a stance on their rights to do so and their status may be affirmed or negotiated by the child.

A primary way that parents get children to act is through directives (Goodwin, 2006; Goodwin \& Cekaite, 2018). Directives are, broadly speaking, actions employed for getting people to act (Goodwin, 2006). Directive language has been studied extensively (Kent, 2011); however, much of this work has been in the service of developing taxonomies based on linguistic form (Ervin-Tripp, 1976), or presumed politeness (Brown \& Levinson, 1987), or degree of explicitness (Blum-Kulka, 1997). Interactional research has, however, shown that directives can take many forms when recognised based on action (Kent, 2011).

Parents orient to deontic matters in the ways that they design their directives to children. Conversation analytic work has examined how directives can be built to orient differentially towards entitlement to direct or request and the possible contingencies acting upon the recipient (Curl \& Drew, 2008; Craven \& Potter, 2010). For example, imperative directives (e.g., "eat your dinner") tell children what to do, rather than ask or propose, with no concern for the possible contingencies impacting the child's ability or interest in complying. In contrast, interrogative directives also can orient to these contingencies and the entitlement to ask (e.g., “do you wanna eat your dinner?”). These different formats show how parents can orient to the need to direct while also seemingly allowing for differing levels of autonomous participation.

Children are sensitive to the ways that directives construct the relationship between themselves and their parents and can respond in ways that enable or resist that construction. The ways that children respond to parents' directives show that direction is not just a one- 
way street with parents making all the decisions about children's behaviour in the home. Children can recognise the entitlement of a parent to direct them (i.e., affirm the parent's deontic status) through simple embodied compliance (Kent, 2012a) or they can negotiate what the parent is entitled to direct them to do or what they are willing/able to do (Aronsson, \& Cekaite, 2011; Goodwin \& Cekaite, 2018). They can baldly refuse to comply, resisting parental control and asserting complete autonomy (Goodwin \& Cekaite, 2018; Kent, 2012a: Kent, 2012b), or they can delay actual compliance in order to present themselves as doing the directed activity but on their own terms (Kent, 2012a).

Parental directive practices and their displayed stance on their children's deontic rights differ cross-culturally. Demuth (2013) showed that, while families in Cameroon tended to use upgraded, authoritative directive forms, German parents tended to use mitigated, downgraded directive practices, downplaying their directive authority and highlighting the children's autonomy. This is demonstrated in the use of activity contracts by Western families (Aronsson, \& Cekaite, 2011). Activity contracts are extended directive trajectories in which a parent issues some directive that is negotiated with the child via upgrades, downgrades, alternative actions, and provision of accounts/reasons until a form of the directive is ratified by the child. These contracts are a way for parents to establish expectations of accountability for the children's future conduct, socialising them into accountability (Aronsson, \& Cekaite, 2011), and orient to the child as possessing rights to understand, and participate in, household matters (Demuth, 2013).

This chapter further develops on the study of parental direction of their autistic children by analysing how a parent directs their child to use the bathroom before going to bed. Analysing how this activity contract negotiation unfolds will show what resources the parent utilises in support of her directive as well as how the child himself contributes to the negotiation by responding to deployed resources. These analyses will also demonstrate how the negotiation of the activity contract is a locus for the negotiation of the social relationship between the two as issues of autonomy, group membership, and what is normal become resources for direction.

The case analysed here features Trevor and his mum comes after they have spent some time after dinner playing a riddle game together on the couch. After the game, Mum proposes heading to bed to read some of their book together before sleep. This episode demonstrates a diversity of strategies Mum uses to direct Trevor to try to use the toilet before 
going to bed. These strategies include negotiating Trevor's autonomy, negotiating categorytied activities tied to the category of Trevor's family, utilising beneficiary status and coercion, and physical shepherding Trevor. These are reported in the order they occurred and were chosen as they represented clear, distinct activities constituting the overarching project. This interaction was selected for analysis here because it shows how issues that raised individually in the previous chapters (issues around autonomy, rights, knowledge, emotion, and embodiment) are all live in the interactional substrate of this sequence. While the overall project and the individual activities are not necessarily explicitly about these issues (i.e., the participants are talking about those things), the actions they accomplish construct and depend upon them. Unlike hypothesis-driven research, which requires high degrees of specification in the phenomena of interest and strict control of variable which may confound the results, these analyses show how these complex social phenomena are visible and live in unconstrained, naturally occurring social interaction.

\section{Analysis}

Throughout this interaction, Mum and Trevor are seated next to each other on their couch, with Mum to the left of Trevor (Figure 1)

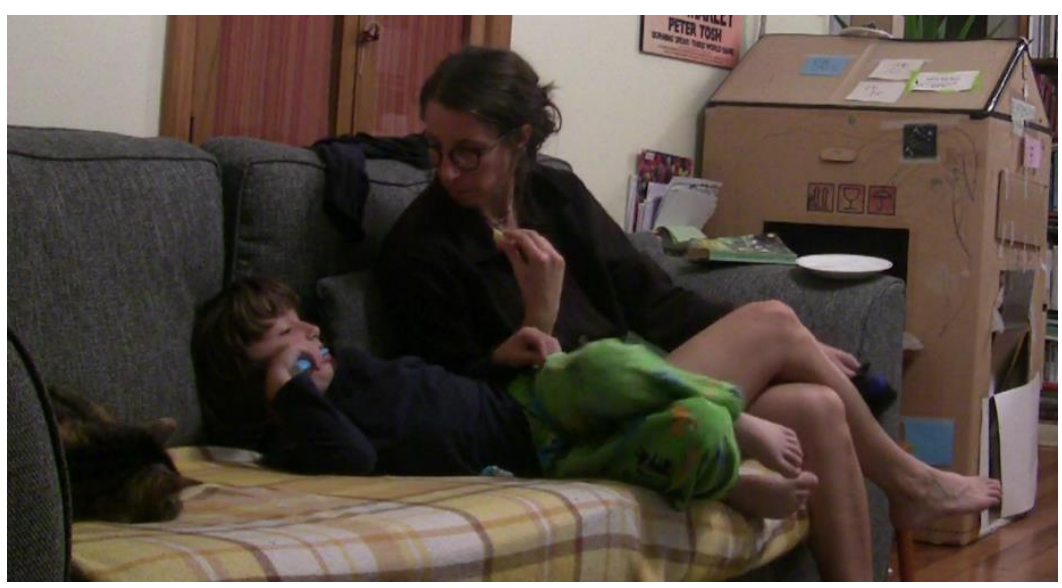

Figure 1. Mum and Trevor seated.

\section{Negotiating Autonomy}

This first sequence demonstrates Mum and Trevor mutually negotiating Trevor's autonomy in the course of Mum's initial directive for Trevor to use the toilet before bed. Mum issues her directive in terms of Trevor's desires and Trevor uses his desires to resist compliance. Before line 1, Mum has made the proposal that she and Trevor go to bed and read a couple of chapters of their book together. Trevor accepted the proposal which led to Mum asking the question in line 1. 
Extract 1

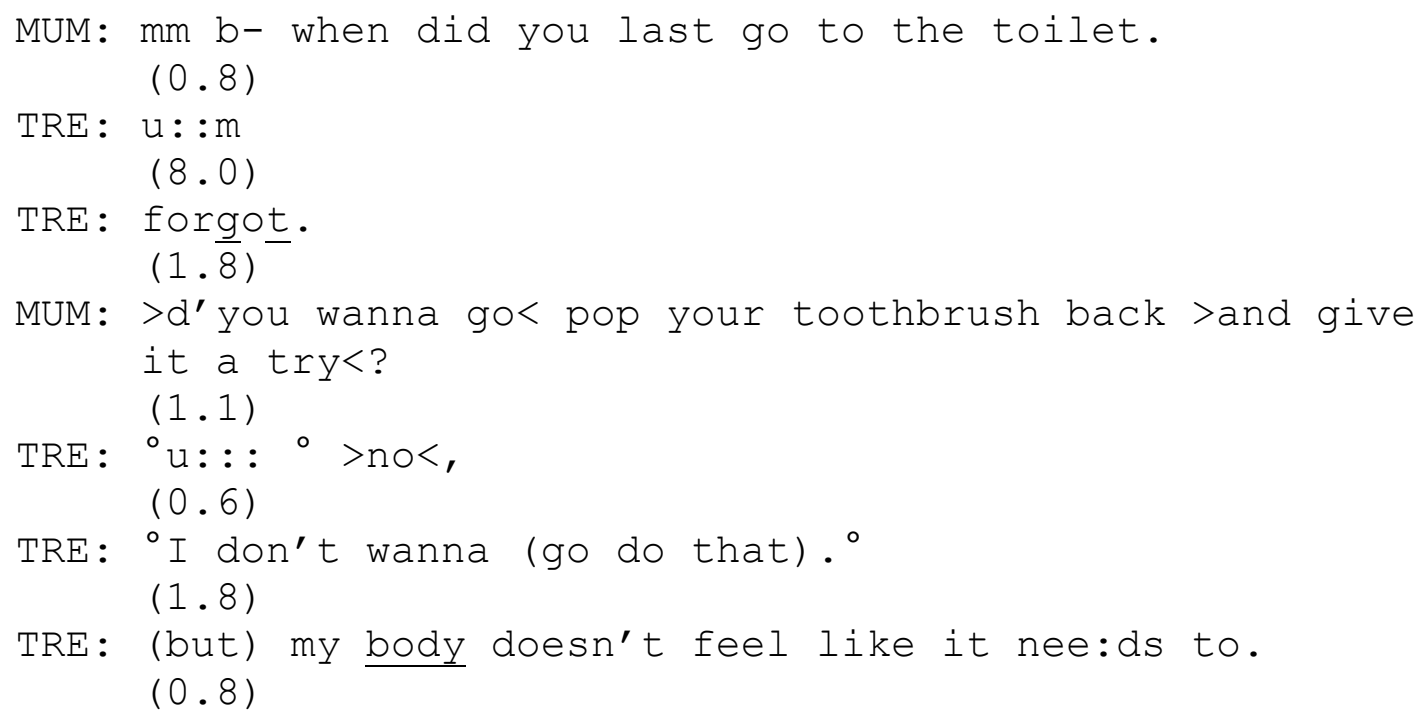

In this extract Mum issues a directive that allows space for Trevor to act autonomously while also telling Trevor what to do. Stevanovic (2018) wrote about first position downgrading incongruence, whereby participants can depend on their turn's sequential position (i.e., first position) and presumed deontic status for the deontic thrust of their turn despite their turn's displayed low deontic stance. Mum's directive at line 7 displays a low deontic stance by orienting towards whether Trevor wants to do as proposed. Analysis of requests and directives has shown that they are designed along a continuum of orientation to both the speaker's entitlement to make the request/directive and to the possible contingencies hindering the recipient's compliance (Curl \& Drew, 2008; Craven \& Potter, 2010). Mum begins with the question-formatted information request " $>$ d'you wanna go $<$ pop your toothbrush back $>$ and give it a try<?" at lines 7-8. This directive is designed with an orientation to a possible contingency that might hinder Trevor's compliance; his desire. By using this format as the initial directive form, Mum provides a space for Trevor to appear to be acting autonomously, based on his own desire, rather than complying with someone else's will. Mum's first position downgrading incongruence move was recognised by Trevor as a directive and not just an information request; however, it did not secure compliance from him.

Trevor's rejections of Mum's directive display a sophisticated awareness of the social work being done in this short sequence. Trevor shows his understanding of Mum's directive as oriented towards his desire in lines 10-14 when he first refuses with "० $\mathrm{u}::{ }^{\circ}>$ no $<$,", explains his rejection on the basis of his desire (as the directive format allowed for) with " $\mathrm{I}$ 
don't wanna (go do that). "’, and further adding “(but) my body doesn't feel like it nee:ds to.”. Rather than just issue a bald rejection, Trevor provides accounts for his rejection. Heritage (1988) wrote that accounts in dispreferred responses tend to be based on inability rather than desire, stating that accounts often have a "no fault" quality that "avoid[s] any threat to the social relationship" (p. 36). Trevor's first account is not oriented toward saving the face of Mum. However, his account does maintain the social relationship on the grounds set up by Mum in her directive. By responding that he does not want to do what was proposed he is in a sense ratifying the stance taken by Mum in making the low-entitlement and high-contingency directive in the first place. His second account is a more typical no-fault style. Additional to not wanting to, Trevor does not need to use the bathroom. As such it is no one's fault that the action does not get accomplished at this point and Trevor gets to position himself as sensitive to the social relationship being constructed by Mum's directive while also resisting blame form non-compliance. Trevor does here what Stevanovic (2018) calls second position upgrading incongruence. His declining and two accounts combat directly the presumed desire proposed in Mum's directive as well as counter the possibility that he might need to use the toilet. As the one that has epistemic rights to his experiences, Trevor can upgrade the incongruence between Mum's deontic stance and his own deontic status over his own behaviour.

This sequence shows how in the moment of telling Trevor what to do, Mum and Trevor become mutually involved in the construction of Trevor's autonomy. Here, Mum presented what she wanted Trevor to do in terms of Trevor's own desires, while Trevor used his own desires to resist compliance and ratify the low deontic stance Mum claimed in her directive turn.

\section{Negotiating Category-Tied Activities}

Extract 2 comes 36 seconds after Extract 1 and shows how Mum and Trevor engaged in negotiating what is expected of members of their family. Mum constructs trying to use the bathroom before bed as a normative feature of their family while Trevor dismisses that construction. This negotiation is done in the service of accounting for Mum's directive. As she ties the behaviour to category membership, Trevor responds by denying that categorybehaviour association. 
Extract 2

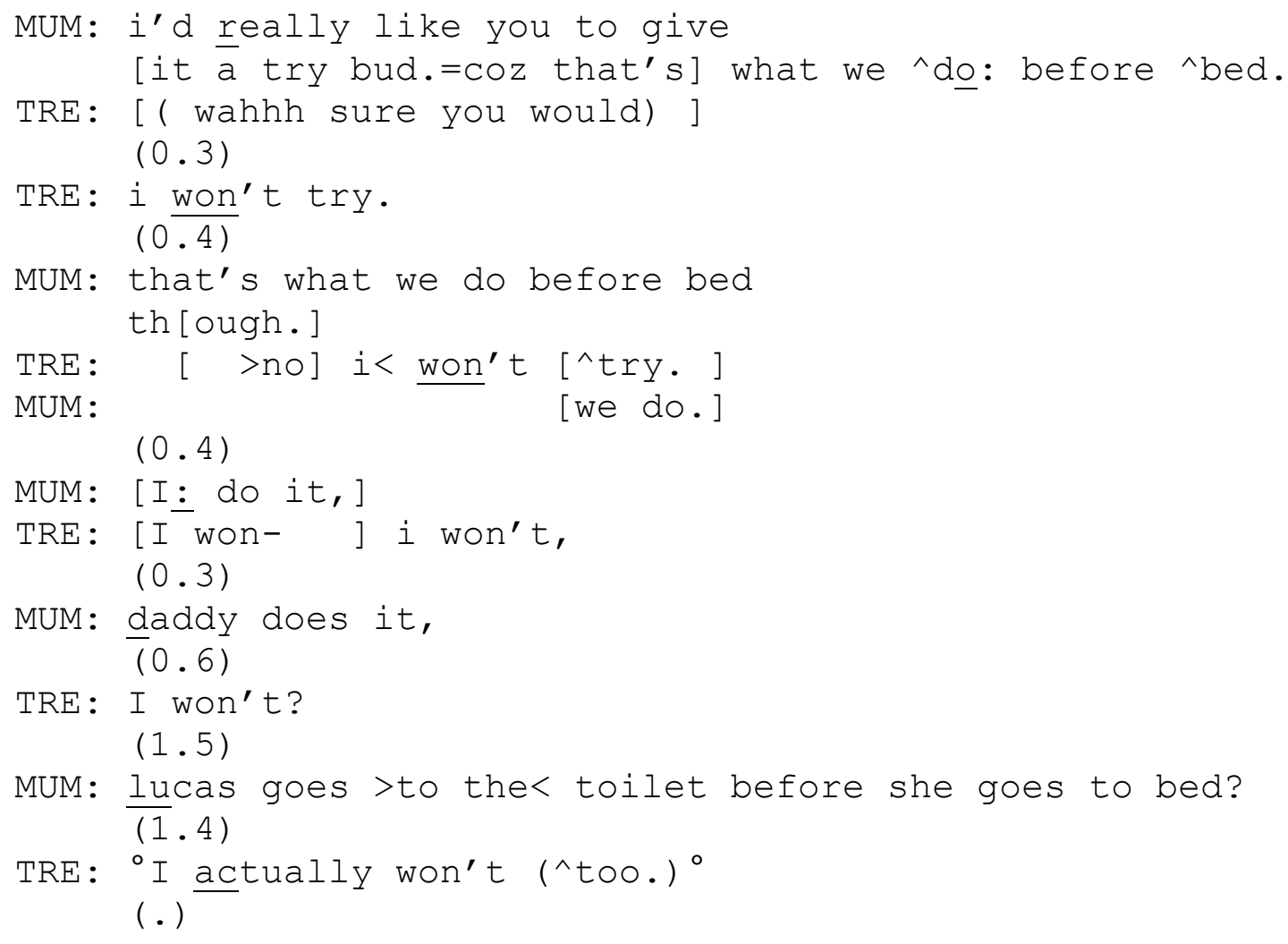

Mum construction of trying to use the toilet before bed is as a category-tied activity. A category-tied activity is a category feature whose link to a category is not taken for granted and thus needs to be explicitly stated (Reynolds \& Fitzgerald, 2015). In line 1, Mum produces her directive for Trevor to "give it a try" before accounting on line 2, saying "coz that's what we ^dㅁ: before ^bed.”. Mum unpacks the collective pronoun "we" (line 7) as including Daddy (line 15) and Lucas the cat (line 19). These are the members of Trevor's household and family. Therefore, Mum is accounting for her directive by setting up trying to use the toilet before bed as an activity all members of the family normatively do.

Mum also accounts for her directive by including Trevor in the group referred to as "we". Not only is it something that the people she mentioned do; it's something Trevor is expected to do as a member of the same category. After Trevor takes a mocking stance at line 3 towards Mum's directive by producing a designedly fake cry and acknowledging Mum's desire for him to give it a try and then rejects it outright on line 5, Mum restates her explanation "that's what we do before bed though". This turn coming after Trevor's explicit rejection shows Mum treats Trevor as a member of "we" and as such his refusal to do something "we" do is counter to what is expected of a member. As a member of the same category "we" he should not refuse. Trevor's response to this at line 9 is to reject the 
formulation before giving his refusal again. His rejection challenges that trying to use the toilet before bed is an activity he needs to perform as a member of the family.

Trevor also continues his rejection at line 21 after the "we" has been unpacked. The "actually" here marks that the not doing it is inapposite to expectations (see Clift, 2001). Mum has just demonstrated how all the members of the family try to use the toilet before bed and so Trevor acknowledges that his refusal as a member of the family is inapposite or unrespectable, but regardless he still will not try. Note that this is not positioning himself outside of the category constructed here; Trevor positions himself within the category but continues to challenge the tie between the category and the behaviour.

This extract demonstrates the negotiation of categories and category tied activities by Mum and Trevor. A couple of different activities are being accomplished here. Firstly, Mum and Trevor are negotiating what it means to be part of their family, with Mum proposing that members of the family can be expected to try using the toilet before bed and Trevor denying that link. Secondly, Mum is using this negotiation in order to issue her directive to Trevor, with Trevor using his own negotiating to resist that directive. Mum uses the expectations set up by category membership (in this case even those expectations that are not normative but are locally made explicit) to direct their children into behaving.

\section{Invocation of Consequences}

In this extract (occurring immediately following Extract 2), Mum provides more reasons for why Trevor might want to use the bathroom now, namely that if he does not go now he may have to in the night when it would be both uncomfortable and annoying and also might leave him prone to attack by a spider that had previously been an issue in the bathroom. Trevor engages with this reasoning, understanding it not just as a separate activity (discussing the spider) but as being in service of Mum's directive, and dismisses it for his own prioritised experiential reasoning.

Extract 3

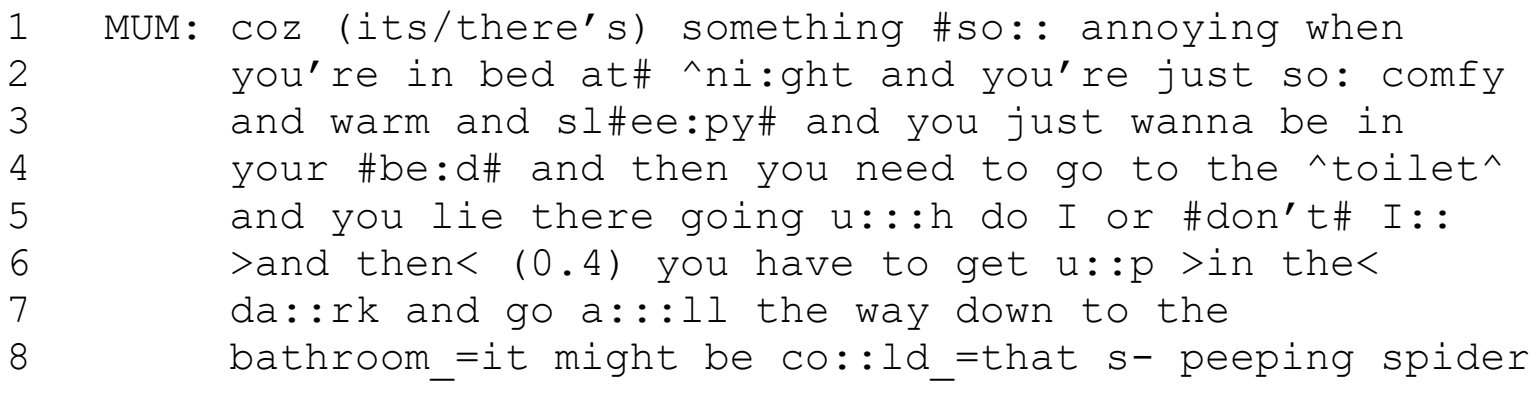




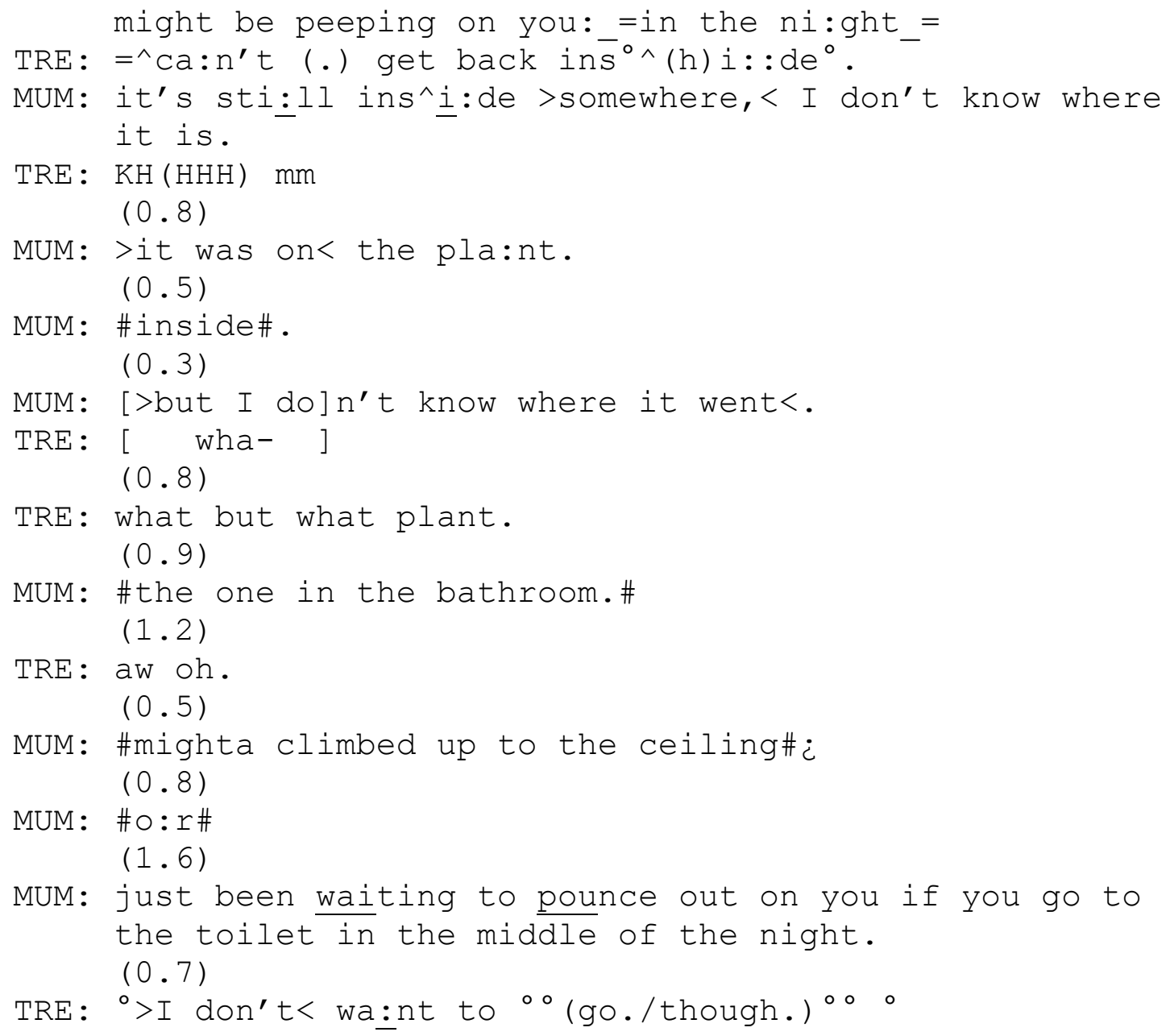

In lines 1-9, Mum is providing an extended, multi-unit turn which outlines reasons for why going to the toilet in the night should seem a less preferable option to Trevor. These reasons include that it will be annoying, you will be warm and sleepy in bed, you will have to deliberate getting up, it will be dark, it might be cold, and lastly there might be a spider. This multi-unit turn is accomplished via common turn constructional methods such as compressing the transition relevance place via turn constructional unit-final sound stretches (“do I or \#don't\# i::" on line 5), latching ("be co::ld_=that s- peeping" on line 8) or suppressing turn-final intonation ("bathroom_" on line 8; Clayman, 2012). This has the effect of giving Mum the space to list all the negatives consequences to not using the toilet nor before bed, presenting it as a wholly undesirably thing.

Mum's reasoning ends with reference to a possible encounter with a spider should Trevor need to get up in the night. Mum's talk presents the spider as not just any spider. Firstly, it is referred to with the demonstrative determiner that, characterising it as a specific spider, not just any spider that could be there. As well, that refers to a spider that is not just 
spatially distant (i.e., in the bathroom), but also temporally distant, having been a consequential object in previous activity. This is made clear in the following lines where both Mum and Trevor appear have dealt with the spider in the bathroom in the past. Secondly, Mum's insertion repair constructs the spider as not just a spider, but a threatening spider, by cutting of production of spider at just "s-" on line 8 and inserting "peeping". Characterising this spider as one that Trevor is aware of and one that might not just be watching, but peeping with its connotations of malicious intent, is a way of building a consequence that is outside of the experiential domain of the other consequences. The spider is not just a feeling or experience of discomfort, darkness, or cold, but is an agentic threat against those who might draw near.

Mum's reference to the spider provides an opportunity for Trevor to redirect the current course of action away from Mum's continuing pursuit of compliance with her directive, and this results in a negotiation of the significance of the spider. At line 10, Trevor responds to the spider reference, topicalising it by recasting it as not a problem because it “^ca:n’t (.) get back ins ${ }^{\circ \wedge}(\mathrm{h}) \mathrm{i}:: \mathrm{de}^{\circ}$." This description of the spider's inability to return inside characterises the spider as outside, and thus not a threat to Trevor in the case of him needing to use the toilet in the night. This diffuses the spider as a resource for Mum's directive.

At this point, Mum moves to re-establish the spider as a threat which would motivate avoiding using the bathroom in the night. She does this by countering Trevor's presumption that it is outside as well as by presenting the absence of the spider as potentially threatening. On lines 11-12, Mum says the spider is still inside, directly countering the presumption imbedded in Trevor's previous turn that the spider is outside. She further adds that she does not know where inside the spider is at lines 11-12, 15, 17, and 19. Her description of her last seeing it on the plant inside re-establishes it as a threat to Trevor as it is inside, but even more so as now it is inside but its specific location is unknown. This creates an uncertainty regarding the danger to Trevor of being caught by the spider if he were to use the toilet in the night. Trevor makes an attempt to rectify this uncertainty by asking Mum on which plant she saw the spider on line 22, but after Mum further establishes the uncertainty around the spider's whereabouts he ultimately abandons his concern for the spider potential implications, returning to his current feeling of need (or lack of) to use the toilet.

This extract demonstrates how the directive trajectory can be supported by drawing on shared-in-common knowledge from the day's affairs. Mum here could take for granted that 
Trevor remembered the spider she speaks of and thus this could become a resource for motivating his action. Not only this, it is a site where events for the day can be recapitulated and provided new meaning in different interactional contexts. Mum and Trevor negotiate the relevance of the spider to their current course of action, with Mum treating it as a potential future threat and Trevor ultimately dismissing it for his own present interests.

\section{Utilising Beneficiary Status and Coercion}

Clayman and Heritage (2014) describe how requests can be formulated to differentially highlight the participants' benefactor/beneficiary relationship. Extract 4 comes immediately after Extract 3 and shows how Mum designs her directive to construct the benefactor/beneficiary relationship between Trevor and herself, respectively.

\section{Extract 4}

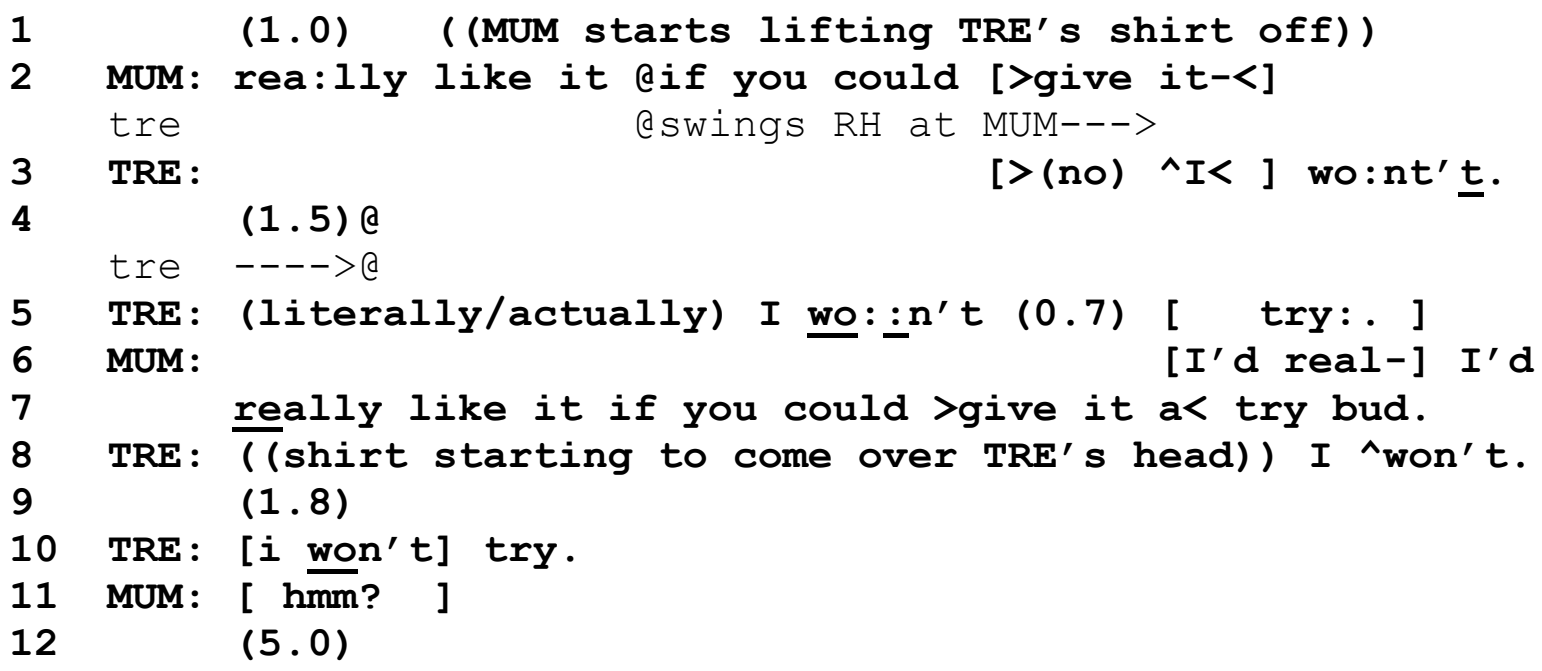

Mum's directive here (lines 2 and 6-7) is formulated as a request where she is the beneficiary of Trevor's benefactive action; trying to use the toilet. In this way, she can portray Trevor as someone who could do a good or nice thing for her. Clayman \& Heritage (2014) write that by formulating the speaker's interests, their turn can be heard as a request. Mum starts in line 2, saying she'd "really like it if you could >give it- $<$ ", projectable as heading towards "a try", and launches this again at lines 6-7 after Trevor's refusal in overlap (lines 3 and 6). Mum refers to what she would like Trevor to do and Trevor demonstrates he hears this as a request to do it at lines $3,5,8$, and 10 with his rejections.

Mum reissues the same request, this time completely, ending with "give it a try bud" at line 7. Post-positioned address terms, like "bud" here, have been found not to do simple person reference or address work, but instead have a personalising function, displaying a 
stance toward the person addressed (Lerner, 2003; Rendle-Short, 2007). As an address term, bud constructs a certain type of relationship between Mum and Trevor, namely one of friendship or friendliness (as bud is a common shortened form of buddy). By this, Mum takes a stance on Trevor's obligations towards her. Favours are implicitly bound to the category of friendships. By constructing their relationship as one of friendship, Mum can produce the moral accountability of the relationship to attempt to secure compliance.

Trevor's rejection of the benefactor role is strengthened by his physically aggressive behaviour (i.e., when he swings his arm at Mum in lines 3-4) which places Mum and Trevor in an unfavourable relationship of dispute, rather than constructs their relationship felicitously. Mum's directive at lines 6-7 takes the same form as earlier by highlighting her interests but beyond just placing the benefactive responsibility on Trevor, she also explicitly categorises him as the kind of person who would do favours (namely a friend), and redefines their relationship from one of conflict to one of kindness.

Extract 5 continues immediately after the 5 second silence at line 12 of Extract 4 and shows how Mum coerces Trevor into being responsible for her negative mood and how Trevor rejects this positioning by ending the conversational sequence.

\section{Extract 5}

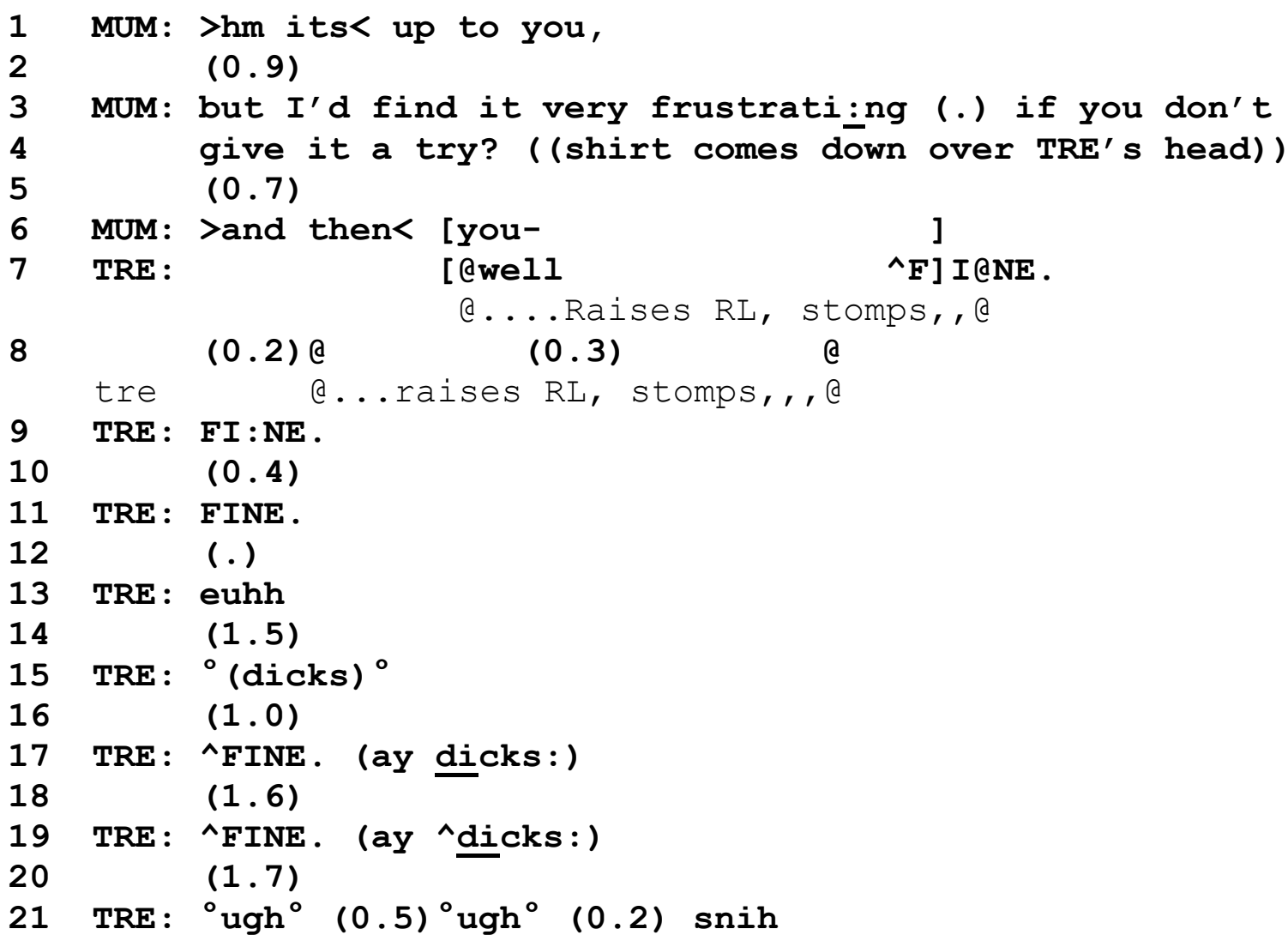


23 TRE: i'm ^not gonna ^try.

$24 \quad(1.8)$

25 TRE: ${ }^{\circ}$ (i want to cry:.) ${ }^{\circ}$

26 MUM: ptch kay maybe you're right \#about doing two tops

27 at one\# $t(h)$ ime, hh

$28 \quad(1.4)$

29 TRE : ye: :ah

30 MUM: myeah

$31 \quad(1.8)$

After rejection from Trevor, Mum pushes her role as the beneficiary of Trevor's action in a way that coercively makes Trevor responsible for her negative feelings. Clayman and Heritage (2014) describe the ways whereby a claimed beneficiary can further their request with reference to the maximised benefits or minimised costs should the benefactor comply. Mum breaks the silence with " $>$ hm it's $<$ up to you" (Extract 5, line 1), which presents compliance as a choice. Mum's turn at lines 3 and 5 add that there are some consequences in choosing; should Trevor choose not to comply Mum would find it very frustrating. Mum is expressing the maximised costs of not complying, rather than the minimised cost or maximised benefit of complying. This focus on her frustration emphasises Trevor's responsibility for her negative emotions. Rather than providing a friendly favour, Trevor is positioned as choosing Mum's mood.

Trevor's response to this emphasis on causing Mum's negative mood is a display of his own negative affective stance while providing the preferred response to Mum's directive. Trevor demonstrates a sophisticated understanding of conversational sequence in his response as he uses this understanding to move to end the directive sequence. Trevor shouts "FI:NE" three times successively (lines 7, 9, and 11). After the last "FI:NE" Trevor makes a guttural grunt sound before a continued series of "FINE" accompanied by swearing (lines 15, 17, and 19). Sterponi and Shankey (2014) found delayed self-echoes like Trevor's repetition of "FI:NE" were common in contexts of diverging projects between interlocutors. In this way the child is able to redirect their interlocutor's attention towards something else, even if only temporarily. Each of Trevor's echoed utterances are exaggerated in their length, tone, and volume and are accompanied by punctuating foot stamping. These marked displays negatively assess the course of action and also end the course of action by providing the preferred second pair part to Mum's directive and reorienting Mum away from her persistent directing. Whether or not he intends to comply (line 23 suggests he does not), providing the 
answer he understands Mum to be looking for is a method for ending the current course of action that upsets him.

These extracts demonstrated two strategies used by Mum and Trevor. First, Mum constructed a relationship between her and Trevor where he was a friendly benefactor towards her. However, that relationship construction gave way to coercion. Second, Trevor was an active participant in this relationship construction, opposing it for a more combative construction, as well highlighting his sequential understanding in the way he managed his response to mum's coercion.

\section{(Incipient) Compliance}

This final extract comes 30 seconds after Extract 5 demonstrates both Mum's and Trevor's use of their bodies in this directive trajectory. Mum uses her hand to physically shepherd Trevor off the couch while Trevor uses his body to resist this shepherding by slumping to the floor. In Trevor's action we finally see his compliance, but it does not come smoothly as he asserts his own independence.

Extract 6

1 MUM: \#>so you're gonna give *@\#it a try, < * $\mathrm{RH}$ on TRE's back--->

tre deans forwards \#fig1 \#fig2

Fig:
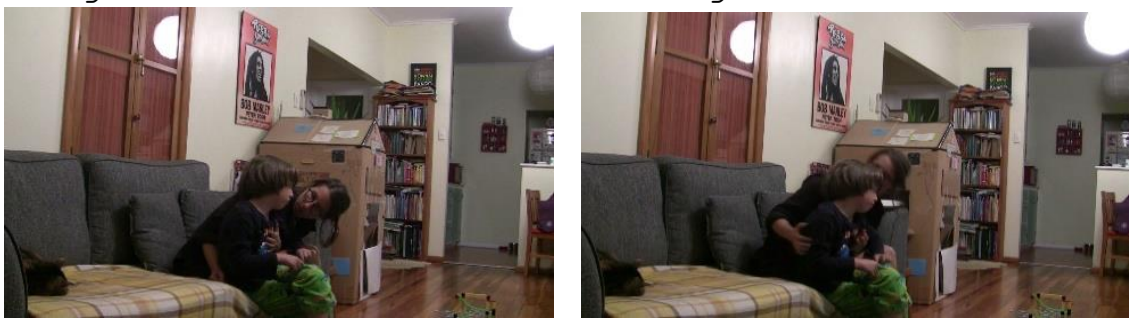

Fig1

Fig2

2 TRE: i won't (.)\# @

Qslides to floor--->

mum

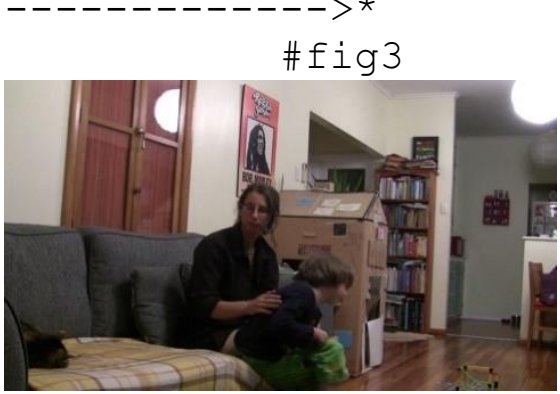

Fig3

3 MUM: I'd rea:1[ly like it] if you ecould bud,

4 TRE : [ @\#OWW] 


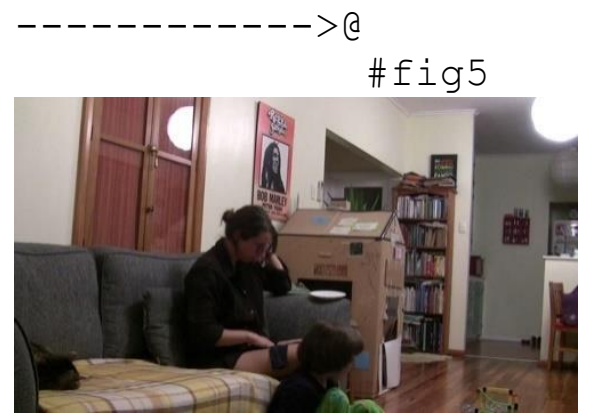

estands up--->

Fig5

MUM: we're going around

[in circle- ]

7 TRE: [ $>$ well $i<$ won' $^{\prime}$ ] actually tr@\#y.
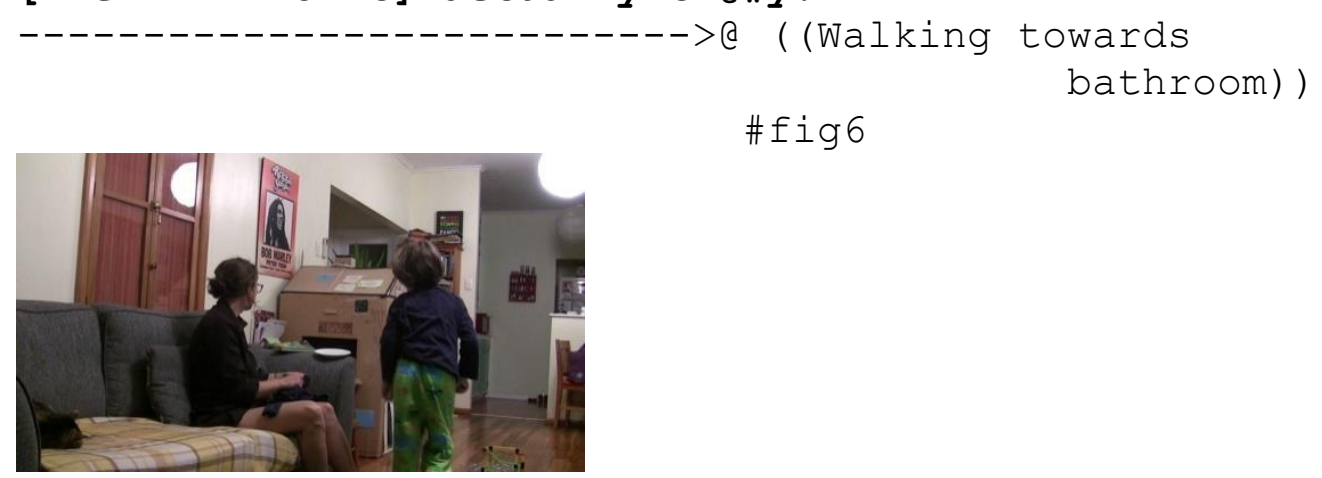

$\#$ fig 6

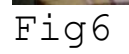

8 MUM: @ we're a going around in circles

$$
\text { tre a..gaze at MUM, , a }
$$

9 here?=[but i'd real]ly like it=

10 TRE: [( )]

11 MUM: =if you would.

$12(0.4)$

13 TRE: @\#^yea:h but I'm |not.

14 (1.3) @\#

deturns to face MUM--->

tre $\begin{aligned} & --->0 \\ & \text { \#fig7\#fig } 8\end{aligned}$

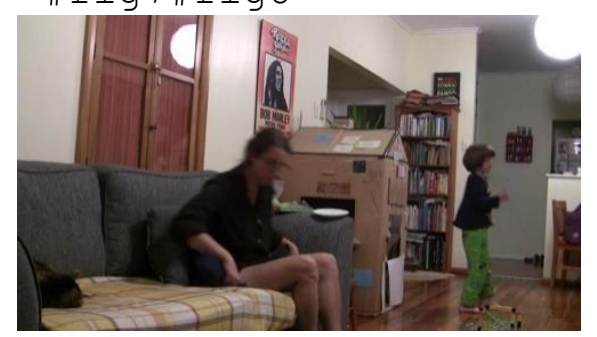

Fig7

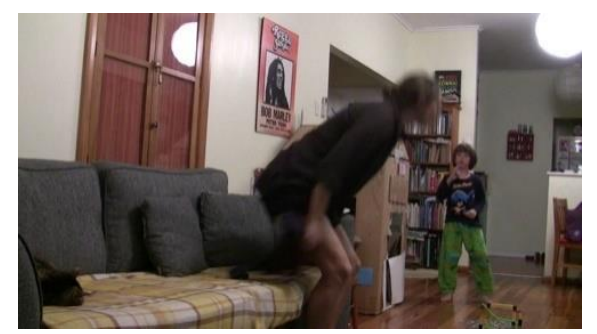

Fig 8

15 TRE: ( (gulp)) $\mathrm{mm} \mathrm{mm}$

$16 \quad$ (3.5)

17 TRE: ^i'm not trying. Mm mm

18 (6.5) ((Bathroom light turned on))

19 TRE: fine. I'll try: : .

20 MUM: tha:nk you 
Just prior to this extract, Trevor provided a minimal acceptance of consequences suggested by Mum for continuing to delay going to bed; He will have less time to read his book. At line 1, Mum demonstrates with both her spoken and embodied conduct that she understands Trevor's acceptance as a move towards compliance. Mum does this verbally with a request for confirmation "so you're gonna give it a try," at line 1 . This turn makes the statement that Trevor is going to try and turns it into a confirmation check with the rising turn final intonation. As well, the initial "so" marks that it is not just a check for confirmation coming out of nowhere; she is confirming the upshot of Trevor's utterance (see Raymond, 2004). This shows her understanding of Trevor's acceptance as possibly projecting his compliance.

While Mum's verbal conduct appears to primarily function as a confirmation of her understanding of the upshot of Trevor's action, her concurrent embodied activity demonstrates she is still oriented towards the directive and attending to the possibility that Trevor still will not comply. This is shown in how Mum positions herself physically as a directive agent in relation to Trevor. Mum's initial position is that of a side-by-side arrangement producing an F-formation (Kendon, 1990). Kendon (1990) describes an Fformation (i.e., facing formation) as the result of an arrangement which produces a space between interactants that they mutually orient to and maintain. As she produces her turn, Mum withdraws her face-to-face contact and reorganises herself into a C-formation with Trevor (Cekaite, 2010), placing herself behind him with her hand on his back. Contrasted with an F-formation where everyone is visibly co-present, the C-formation is defined by an asymmetry, in that one person retains exclusive rights over access to their own bodily positioning while also claiming rights over others' (Cekaite, 2010). Mum moves from mutual interaction, to leaning back behind Trevor (removing her upper body and face from Trevor's view) and raising her hand to his back in order to push him, utilising her new position behind him as a directive tool (fig 2, line 1). This movement from a position of mutual engagement to one of control during her spoken request for confirmation changes its tone. Rather than being a request for information that requires answering with a yes or no, this turn becomes a further directive. Mum is not concerned with getting a verbal answer and displays a strong interactional preference for embodied compliance.

Where Mum uses her body to further her directive trajectory, Trevor uses his to continue rejecting it before ultimately complying. His embodied conduct is not just rejection, however, but also constitutes his stance towards being told what to do. Trevor first turns his 
forward movement into resistance as, instead of leaning forward and standing up, he slips off the edge of the couch onto the floor while saying that he won't try. His landing on the floor is punctuated with "oww" at line 4, overlapping with Mum's "I'd really like it if you could bud", which paints his physical experience as his primary concern over Mum's continued talk.

Once Trevor stands up at line 4, he continues to make verbal rejections to the directive at lines 7, 13, and 18 whilst also slowly moving in the direction of the bathroom. This sequence of moves towards the bathroom despite claims he is not doing what Mum asked before actually complying looks similar to incipient compliance (Kent, 2012). Incipient compliance involves a display that some complaint action is "in the offing" (Kent, 2012, p. 723) despite their not actually complying. In our case, this segment is the first time Trevor makes an embodied move off the couch and physically in the direction of the bathroom. This embodied move can be read as a precursor to actual compliance (i.e., going to the bathroom and trying to use the toilet). Kent (2012) explains that incipient compliance moves can be used to buy time between the directive and the compliance, weakening their direct sequential relations. When the compliant action is ultimately completed it is unclear whether it was done as a compliant response to a directive, or just a first move, initiated by the complier. Trevor orients to this feature of incipient compliance as he seems adamant to indicate that, despite appearances, he is not going to go to the bathroom. Keeping up this defiance while bodily moving towards compliance helps to construct Trevor as an autonomous agent when he does ultimately make it to the bathroom. He is doing what he was told but not because he was told but because he chose to, on his own time.

\section{Summary}

This analysis demonstrated how a mother and autistic child oriented to relational concerns in negotiating an activity contract. It explicated a directive trajectory in which a mother attempted to get her autistic child to try using the toilet before bed. Mum deployed practices for gaining compliance from Trevor including the use of interrogative requests and imperative directives oriented to Mum's entitlement and/or Trevor's autonomy, locally tying using the toilet before bed to family membership and challenging that tie, emphasising Mum and Trevor's roles as beneficiary and benefactor in order to make Trevor responsible for Mum's feelings, and use of touch to shepherd Trevor towards compliance while Trevor uses his body to comply on his own terms. In the course of this interaction, Mum showed her openness to Trevor's resistance and the negotiation of his own future conduct (as per 
Demuth, 2013). Using these varied practices in an effort to gain compliance from Trevor treats him as a agentic social actor and gives him the space to negotiate his participation in the ongoing action. The fact that Mum attempts to secure this compliance through multiple means (regardless of some of them relying on constructions of their relationship that minimises his autonomy) produces the relationship between the two as one that depends on mutual participation.

The analyses also showed Trevor's sensitivity to the strategies Mum employed and highlighted how he asserted his own autonomy with accounts in rejecting directives, negotiated the requirement that he try to use the bathroom before bed based on his family membership, responded to coercion by shutting down the interactional sequence, and used his body to comply on his own terms and demonstrating his awareness of his observability by Mum. As such, analyses demonstrate the sensitivity both participants showed to the local interactional order organising the different activities which made up this directive sequence. The way he responds to Mum's different directing moves presents himself as adopting a selfdirecting stance towards himself and this stance is moderated in response to Mum's contributions. Trevor does not just respond in the negative to Mum's moves. He provides explanations, negotiates the expectations of him based on group membership, weighs up consequences against each other, and uses his body to enact his ultimate choice and production of his autonomous self.

The explication of these different approaches used by both Mum and Trevor in negotiating this activity contract highlight an important point about mundane parenting activities. While parents commonly report that directing their autistic children to do things is difficult, this chapter shows how such an example of a difficult case is more than just a child resisting a directive. Issues of Trevor's autonomy, his relationship to himself and to Mum, affect and knowledge are managed throughout the accomplishment of this activity contract negotiation. An appreciation of the kinds of things that are done in the service of a directive changes these moments from just mundane parenting acts to complex social work.

This chapter shown how both cognitive and sensorial features commonly associated with autism are managed in the course of an everyday parenting exchange. The next, and final, chapter will conclude this thesis by drawing together the analyses of this and the previous two chapters in order to speak to the bigger issues around autism and neurodiversity in social interaction. 


\title{
Chapter Seven: \\ Respecifying Autism: General Discussion
}

\author{
"The aspects of things that are most important \\ to us are hidden because of their familiarity \\ and simplicity. (One is unable to notice \\ something - because it is always before one's eyes.)"
} (Wittgenstein, 1958, p. 50e)

In this chapter, I will review and synthesis the findings of the previous empirical sections. A respecification of autism as an accomplished social category will be discussed. The findings of the empirical chapters will be reviewed briefly and discussed in terms of their implications for both discursive psychological research on the accomplishment of social phenomena and autistic people's social practices in interaction at home. As the findings of this research describe the sophisticated social competence accomplished by the children analysed, the relevance for the categories of autism and autistic child for understanding their accomplishment of interaction is unclear. I claim that, rather than presuming noticeable social phenomena in their interactions are the result of autistic pathology, a variety of things in the social environment contribute to their competence and the achievement of intersubjectivity with their family members.

Dinishak's (2016) critiques of a deficit view in understanding autism were that the deficit model meant leaving autism with only a negative identity in comparison to neurotypicality, and it emphasised quantitative differences rather than qualitative ones. This thesis uniquely brings together the fields of critical autism studies, neurodiversity studies, and ethnomethodology/conversation analysis/discursive psychology. The previous chapters show how many of the concerns of autism and neurodiversity researchers and theorists are amenable to a kind of analysis that is not reductionist, individualist, essentialist, or pathologising. Using conversation analysis within discursive psychology to analyse the interactions had by autistic children has enabled abandoning a concern for deficits. These analyses have taken for granted that interactional intersubjectivity is managed and achieved in interactions in which autistic people are parties and rejected any concern for comparing how these participants compare to other, neurotypical children in interaction. With 
conversation analysis, autistic children can be seen as social agents negotiating their world with their families.

After providing my respecification of autism as accomplished membership category drawing on Garfinkel's (2002) critique of category use in social sciences, the findings that support the claims of competence will be summarised and discussed in relation to previous literature. Following this, the body of work as whole will be discussed in order to explain why autism does not appear to be a relevant category for understanding these interactions (from both the perspective of the analyst and participants). Lastly, some ways that this research could be extended will be described. I will provide some possible targets for future analysis and explain why including autistic participant researchers would benefit the research.

\section{Respecifying Autism}

In analysing instances of these children's talk, I have said very little regarding autism. In fact, without context, it might appear that these children were neurotypical. This might (and has in data sessions and conferences) prompt people to ask, "where is the autism?". How do we reconcile the fact that the children who participated in this research have received Autism Spectrum Disorder diagnoses, however do not appear to be very autistic, as might be expected from the research and autobiographical literature? By that, I mean that the interactions analysed do not appear to be disrupted by what are commonly thought of as features of autistic social dis-/ability. The way to address this requires an alternative understanding of what it means to be recognised or categorised as autistic, and what social significance that category might have.

The ethnomethodological approach taken in this thesis reverses the common way of thinking about categories like "autistic". Garfinkel's (2002) major critique of the social sciences was that they tended to treat social categories as a priori facts about people that could have causal impacts on their behaviour (e.g., one IS autistic and therefore one acts a certain way or experiences a certain thing). His response to this was to treat categories and membership in them as real, but largely constructed. Rather than the category membership having an impact on the person's conduct, the person's conduct was justification for categorising someone. This kind of reasoning about categories was further explicated in his work with a transgender woman, Agnes, as he described the ways the gender categories were managed in interviews with her (Garfinkel, 1967), as well as later in the form of Membership Categorisation Analysis (Sacks; 1967; Stokoe, 2012). 
Under this framework, it is more appropriate to say that the interactions analysed here did not involve autistic children, but instead children who had as some point, for some reason, been called autistic on the basis of their conduct ${ }^{9}$. This is not to deny that these children or other autistic individuals are impacted their differences (or more rightly, the interaction between their differences and society), or that people should change how they categorise themselves or others (noting that using the category of autistic for oneself has important individual, social, and political implications; Chapman, 2020). Instead it is to recognise that who is rightfully called autistic, by who, and for why, is an interactional problem managed by participants.

The ethnomethodological, discursive psychological, and conversation analytic approach to autism and autistic people is concerned with how social interactants themselves make themselves understood (or not) and understand others (or not). By bracketing off concerns about whether or not autistic people possess certain kinds of individual differences, their actual conduct can be analysed for what it accomplishes (and how) in the moment. Doing so has clearly demonstrated the minimal relevance of autism as a category for sensemaking in autistic children's household interactions. The following sections will briefly review the findings of this research, discuss some of the ways they fit within previous literature, and consider what they add to understanding of the broader, relevant psychological concerns.

\section{Accounts as Complex Orientation to Others in Interaction}

Autistic people are typically characterised in the psychological literature in terms that suggest they have little ability to engage (or interest in engaging) with other people's thoughts, feelings, knowledge, or beliefs (Kanner, 1943; Baron-Cohen, Leslie, \& Frith, 1985). One explanation for this is that autistic people possess a deficit in Theory of Mind (Baron-Cohen, Leslie, \& Frith, 1985). In fact, this proposed deficit in conceptualising mental states is also suggested to extend to concerns about the Self and the autistic person's own mental states, contributing to a difficulty in distinguishing Self from Other (Lombardo, \& Baron-Cohen, 2010). Studies of this purported Theory of Mind deficit tend to rely on experimental tasks in contrived settings. As a result, the literature on how autistic children actually manage social psychological concerns is largely decontextualised and reveals a

\footnotetext{
${ }^{9}$ Though, it is important to consider that this, nor any other characterisation, is not unproblematic. For example, categorising them as children also requires imposing an identity upon them through which we (as analysts or lay observers) further analyse and interpret their conduct.
} 
picture of how autistic people may act not in naturally-occurring social settings, but instead in contexts stripped of interactive support.

Studying the natural social interactions of autistic children in their homes made clear that they commonly provided explanations for their behaviour. Providing an explanation for one's behaviour demonstrates some concern with how behaviour will be recognised and understood by other people. Inspecting places in the data where accounts might be expected on the basis of previous conversation analytic work (i.e., in dispreferred second pair parts and first pair parts like requests or complaints) showed that the children provided many accounts in exactly these sequential positions. This suggests that not only were the children concerned with how their behaviour would be recognised by other people, they themselves recognised some of the normative locations for accounts, suggesting an understanding of normal expectancies operating in those interactional environments. This builds on work by Ochs (2015) who analysed an autistic child's embodied conduct in the school playground, showing how the child demonstrated a corporeal reflexivity, or an understanding that her body was available for others to observe. By explaining their behaviour, the children in this study showed the same concern, that their conduct is available for others to respond to, and thus sometimes conditions on appropriate responses (from the child's perspective) may need to be set.

The children's accounts in second position were responsive to implications about themselves or their behaviour embedded in their interlocutor's first pair part they were responding to. In producing a first pair part, the speaker constructs the conditional relevance of a certain kind of response (Schegloff, 2007). By doing so, it characterises the recipient as being in a certain position to make the expected response. Providing an account when giving a dispreferred response to that first pair part manages the negotiation of that characterisation, and, as such, is fundamentally other-oriented. When an autistic child provides an account for their provision of a dispreferred (or otherwise inapposite) response, they challenge something about the way the first pair part speaker has characterised them (i.e., challenging their knowledge about, or understanding of, the second pair part speaker or something about their conduct). In this way, the provision of accounts in this sequential position by the children demonstrated their concern with other people's knowledge or understanding of them or their behaviour. 
The children's accounts in first position demonstrates a similar other-attentiveness. Where accounts in second position are designed to manage characterisations of the person or conduct embedded in first pair parts, accounts in first position pre-empt such characterisations that might come from interactants in second position (Antaki, 1996). By providing accounts before others can respond to, or evaluate, them or their conduct, the children characterised themselves as observable objects in the world, available to others for inspection, while also addressing the possible conclusions that people might draw about the significance of their conduct. Descriptions construct the thing that they describe as well as attending to specific possible, or actual, alternative descriptions (Edwards, \& Potter, 2005; Edwards, 1997). In that same way, by characterising themselves or their conduct, the children show themselves to be concerned with particular possible ways of recognising their action as they try to counter exactly that recognition.

Two of the children produced a specific construction of behaviour and its causes in the process of addressing other interactional issues. What this shows is that these children have been socialised into a particular method of accounting for their behaviour which involves the culturally common-sensical ordering of the relationship between behaviour, the mind, and the environment (Potter, Edwards, \& Wetherell, 1993). While all the other cases described in Chapter Five also demonstrate children's practical reasoning about their behaviour and its relationship to the local sequential context, very few cases in the collection involved mental state terminology or explicitly rendered the mind or internal states as part of the relationship. Although not required for explaining all behaviours, references to internal states (like thoughts, feelings, or internal drives like hunger) by autistic children in explanations of their behaviour accomplish specific things. In the cases presented, the accounts involving mental state terms characterise the reasons for the speaker's behaviour as motivational, internal, and not accessible to observers. This is similar to how formulating something as what one thinks/thought presents that thing as coming from the particularly position of the speaker (Barnes, \& Moss, 2007).

My work expands upon previous conversation analytic research on accounting in children's talk. Sterponi (2003) showed that children in her research were sensitive to the ways that they were called to account for their conduct by their parents. That finding is extended here by showing that children attended to the accountability of their action without necessarily being explicitly called to account, and do so despite an autism diagnosis. Sterponi (2004) also analysed autistic children's moral and accountability discourse, showing how 
children constructed others' conduct as transgressive and complained about it, used previous condemnations of behaviour to explain current conduct or rebuke complaints, and constructed their own behaviour as rule-oriented. As well, she demonstrated the socialisation of an autistic sibling into accountability via the observation of and deployment of their sibling's previously-used account. The autistic children in this study can generalise their accounting ability such that their accounts are designed to fit within the specific, local environment of the accountable action. Where Sterponi $(2003 ; 2004)$ show the children studied as engaging in moral discourse, my analyses build on this work by linking the kinds of processes thought to be implicated in Theory of Mind-driven behaviour such as reasoning about one's own and others' knowledge and reasons for acting to ethnomethodological notions of accounting, reflexivity, and category/action ascription.

My analysis of accounts showed that the children were able to build accounts in at least some of the different ways that neurotypical individuals can. Accounts can be more or less obviously marked, relying differentially on resources like using because as a causal clause marker (Schiffrin, 1987), continuous prosody to link a turn constructional unit that accounts to the thing it accounts for (Couper-Kuhlen, 2012), or sequential organisation (Heritage, 1988). I found the children produced accounts using at least because and sequential position. Being able to rely not just on clearly marked linguistic resources but sequential ones as well strongly implicates these autistic children as members of a community of interactors (Garfinkel, \& Sacks, 1970) whose action, and action recognition, depends on sequential turn-by turn talk.

This analysis of autistic children's explanations of their own behaviour constitutes an alternate way of considering autistic people's social skills outside of a Theory of Mind framework. By showing how these children orient to others' knowledge and understanding via their attending to local, sequential social action, I have demonstrated how the notion of a cognitive capacity to theorise about minds is not needed to explain why and how these children act.

\section{Engaging in, and Avoiding, Social Touch in Interaction}

While touch has been increasingly examined as a sensory domain experienced differently by autistic and non-autistic people, affectionate touch and how autistic individuals manage their engagement with it within a course of unfolding social interaction has not been examined in detail. Studies on autistic people's experiences of touch have typically relied on 
methods which gather information on autistic people's responses to touch in decontextualized experimental settings (Blakemore, et al., 2006; Cascio, et al., 2008) or post-hoc reports from autistic people or their significant interactional partners (Vostanis, et al., 1998; Clifford et al., 2012). The impact of this is that the understanding of the intersection of touch and autism is focused primarily around touch as a sensory modality, and that touch avoidance or aversion is understood as primarily resulting from the discomfort from, or disinterest in, the sensory experience (Smirni, et al., 2019; Hilton, et al., 2010), ignoring that touch is also an embodied resource for doing social activity.

In Chapter Five, I addressed the issue of social touch avoidance/aversion by autistic children. I looked at how moments of social touch involving embraces (or requests/invitations to embrace) were managed as social interactional phenomena. These analyses showed that at least one place where social touch avoidance occurs is when the children are already doing something else that would be hindered by the touch. This suggests that autistic children's touch avoidance should not always be considered in terms of sensory sensitivity but in terms of the broader social ecology in which that sensory experience happens. Firstly, some cases of non-problematic embraces were analysed, demonstrating both a child- and parent-initiated embrace. Both of these embraces served as environments for some action; the first provided a moment of physical intimacy in a head-to-head formation (Cekaite, \& Kvist Holm, 2017) designed for both parties to be singly focused on the action at hand, the second provided a space for mutual engagement in another task (playing a game together). Secondly, cases of problematic embraces were analysed. These embraces included ones where invitations/requests were denied, where the embrace was forcibly withdrawn from by the child, and where the child negatively evaluated the invitation to an embrace. A significant difference between the problematic and un-problematic cuddles was that, while the unproblematic cases occurred in between courses of action (either at the projectable end of an extended interaction or at the beginning), the second cases all involved some misalignment between the two parties. Whenchildren were already engaged in some other course of action, they prioritised that over parents attempts to initiate embraces with them.

The results of this study show how in the course of unfolding social interaction, touch is not just treated as a feeling but also as a space for a variety of activities; the mutual sharing of affection, engagement in a joint activity or game, showing who you are to others and who they are to you. At the same time, touch can also be something that disrupts or is otherwise 
unwelcome as a locus of social action. Touch is rightly more than just a sense; touch is a resource, an action, an environment, a relationship, a meaning.

This study showed that affectionate touch plays a social role beyond just being a sensory experience for the individual being touched. It also showed how the rejection or avoidance of touch is not just about avoiding an unpleasant sensory experience. Researchers explain autistic children's aversion to touch with reference to the idea that autistic people experience touch negatively (Smirni, et al., 2019). While there is no denying that the certain kinds of tactile sensations are aversive (e.g., pain), the cases of touch aversion in my data show that it is not necessarily the case that autistic children's touch aversion or rejection should be seen as principally the result of "symptomatic" or different sensory experience. Instead, individual cases of touch aversion or rejection should be understood on their own terms as something that arose within an unfolding social context. Touch aversion by autistic children can be driven by aspects of this social context (like the perceived disruptiveness of the touch to the child's own ongoing course of action) and touch aversion based on these extra-individual, social concerns should reasonably be understood and treated differently from touch aversion on the basis of sensory experience.

Affectionate touch is not just a matter of positive or negative sensory experiences to the autistic children involved in this research. To these children, affectionate touch in the form of embraces embodied emotional and deontic relationships, constituting the affective tone of the actions they participated in around or within the embrace. Chapter Six also expanded upon multimodal analyses of touch in social interaction more generally. Previous studies have looked at affectionate touch in the contexts of consoling upset children, farewells, reconciliation, and celebration (Cekaite, \& Kvist Holm, 2017; Cekaite, \& Bergnehr, 2018; Goodwin, 2018; Goodwin, 2020).

These previous studies of embrace have tended to focus on how affectionate touch in the form of an embrace is accomplished. Chapter Five begins from this point in the first two cases analysed as cuddles that are actually accomplished, but then focuses on how cuddles that are requested (or in some other way set up) are specifically not accomplished or their accomplishment is impacted by some other factor. Goodwin (2020) hints at these occasions, saying "Hugs need not run off as interactively attuned... A hug invitee has options for how to proceed" (p. 38) before briefly describing a case where a child actively resists a cuddle and another where the same child negotiates the cuddle by agreeing to "just a little one". My 
analyses of cuddle avoidance expand on the description of how cuddles are avoided or negotiated by drawing on data internal evidence to provide an explanation for why a resistant course of action was taken. For example, there is data internal evidence in Extract 3 of Chapter six for the idea that the resistance to the cuddle was due to its proposal as a conflicting course of action from that which Aidan was already engaged in. By making these links, I have described more than just the unfolding of the cuddle itself, but the unfolding of the broader cuddle/resistance context.

\section{Negotiating Activity Contracts}

Parents commonly report that getting their autistic children to participate in household activities is difficult (O’Nions et al., 2018; Ooi et al., 2016) but there is very little research on how exactly parents attempt to secure compliance from their autistic children. The negotiation of an activity contract between Mum and Trevor played out through a variety of different smaller activities oriented towards getting Trevor to use the toilet before bed. These different activities involved the negotiation of Trevor's bodily autonomy and knowing when he needs to use the toilet, negotiating category membership and what behaviours membership in the family makes expectable, managing a benefactor/beneficiary relationship whereby Trevor is doing Mum a favour, and the management of physical compliance. Each of these individual moments were described with reference to how they progressed or pushed back on Mum's directive.

The findings demonstrated that parenting, at least in this household, is not just a onesided affair. In line with Demuth's (2013) discussion on European mothers' directives, Mum's attempt at securing compliance depended on negotiation in which she gave reasons why Trevor should comply, and she was receptive to Trevor's rejection of the directive trajectory. While Mum oriented to her deontic authority via continually issuing directive first pair parts, Trevor was given, or claimed for himself, a certain amount of deontic space, with both parties actively participating in the directive trajectory. This is consistent with approaches that emphasise the mutuality of social participants' socialisation (Pontecorvo, Fasulo, \& Sterponi, 2001).

This work advances the conversation analytic literature on directives in parenting by demonstrating some of the resources available when making a directive beyond the design and position of the directive utterance itself. Directives do not stand in isolation. They can be part of an overall course of action geared towards acquiring compliance (Aronsson, \& 
Cekaite, 2011; Goodwin \& Cekaite, 2018). The negotiation of compliance is itself a setting within which other important social concerns are managed. In this case, compliance is not just an issue of scheduling activities or material consequences. Who Trevor and Mum are to each other are also at stake. The overall activity builds Trevor as a rational agent via Mum's provision of reasons and justifications and not just demanding (Goodwin, \& Cekaite, 2018), but the specific things reasoned about include further specifications of their relationships together in terms of responsibility, family ties, and their meanings.

This study highlights future research topics that could be addressed. This case showed an activity contract negotiation in the course of bedtime preparation. Sirota (2006) wrote that transitions to bedtime preparation activities are a common site of negotiations and parents of autistic children report bedtime routines are especially difficult (O'Nions et al., 2018; Ooi et al., 2016). It would be worth investigating these routines in families with autistic children with a larger collection study to establish where difficulties might lie and to explicate parents' practices for resolving them. Parental directive practices and their orientations to children's autonomy differ cross culturally (Demuth, 2013). While this case demonstrates a mother treating her autistic child in the manner typical of Western families, it would be important to establish whether this differs along the same cultural lines or if it differs according to the differences in support needs of autistic children.

This study analysed a single, unfolding moment involving one mother and one autistic child. Consequently, these findings are not generalisable to any other directive trajectories in different contexts or involving different people. It is also not feasible to generalise Trevor's methods to other autistic children. This case study does, however, demonstrate a selection of possible local interactional practices that were used in the negotiation of an activity contract, highlighting that these moments can be complex with a variety of social, relational implications at stake in the completion of routine, household activities.

\section{Being Autistic in a Neurotypical World}

Attributing membership with a psychiatric diagnosis, whether by doctors or by lay people, is something that is done in order to make relevant or justify a next action or treatment in the face of disturbing conduct (Coulter, 1979; Moncrieff, 2010). In ethnomethodological terms, category ascriptions can make sense of, or account for, disturbing conduct on the basis of predicates (or features or behaviour common-sensically linked to the category; Sacks, 1967; Stokoe, 2012), which would then afford some next 
interactional or procedural move (e.g., dismissing the talk of something categorised as psychotic, or committing someone categorised as an addict to a therapeutic program). To say that the participants of my research do not appear very autistic speaks more about us onlookers to their conduct in that we do not find it necessary (for most of the time) to explain their behaviour as deviant. So, why is that? Why does the conduct of these children not draw our psychiatric labelling?

A possibility for why "autistic" appears an inappropriate description of these children's behaviour might be that they are well supported by their interlocutors to progress the interactional activities they are engaged in. One reason for autistic children's interactional competence could be the support of generous interlocutors (Ochs, \& Solomon, 2005). Neurotypical, or otherwise relatively- and relevantly-competent, family members can scaffold autistic people's social participation, for example through the recipient design of first pair parts like questions (Kremer-Sadlik, 2004; Rendle-Short, 2014). This possibility is also supported by recent research outside of conversation analysis suggesting that interactions between autistic/autistic and autistic/non-autistic might unfold differently on the basis of different expectations about interlocutors. Autistic individuals do not necessarily accomplish all interaction actions the same way as each other (i.e., autistic individuals are not necessarily able to recognise the emotional expressions of other autistic people; Brewer, et al., 2016), but autistic dyads in interaction have been shown to generously assume a shared knowledge with each other and have a low expectation for the tight coordination of action (Heasman, \& Gillespie, 2019; Sng, Carter, Stephenson, \& Sweller, 2020). This means that interactional problems deriving from a momentary lapse in intersubjectivity do not necessarily arise commonly. As well, research shows neurotypical interactants presume autistic interactants' are more egocentric than they are (Heasman, \& Gillespie, 2018) and prefer other neurotypical interaction partners over autistic ones, whereas autistic individuals either have no such preference (Morrison, et al., 2020) or prefer other autistic interlocutors (Crompton, Hallett, Ropar, Flynn, Fletcher-Watson, 2020). Recognising that interactional competence is a function of the entire local social ecology (including other interactants) is a significant step away from presuming that competence in individually localised in people's social brains.

Alongside the interactional scaffolding (or support in accomplishing actions) that appears in supportive families, different interactional settings and the activities commonly completed within them often have different interactional expectancies tied to them (Garfinkel, 1963). For example, the interactional demands children face at school are very 
different from the interactional demands they face in the home with family (Fleer, \& Hedegaard, 2010; Florio, \& Shultz, 1979). There are more and a wider diversity of people/temperaments to manage as well as reduced flexibility in the kinds of activities the children are expected to be engaged in and when they can do them (e.g., eating at set break times, sitting and working/reading for extended, predetermined periods). These different interactional expectancies might be what turns up the signs and symptoms of autism (Lynch, 1984). This has been shown in conversation analytic studies of autistic children participating in formal assessments of cognitive abilities (Maynard, \& Turowetz, 2017; Korkiakangas, Dindar, Laitila, \& Kärnä, 2016). These studies showed that some of the children who failed aspects of these standardised tests were orienting to everyday conversational features in the manner of the test delivery. For example, Maynard and Turowetz (2017) show that a child treats items in a test as if they were sequential prompts for a narrative telling rather than isolating them as individual items as the tester expects. Because they were treating the task items as produced by the professional tester as if they were part of a conversation, they did not provide the responses sought by the task items. Consequently, their conversational competence actually got in the way of them achieving a score indicative of that competence (Maynard, \& Turowetz, 2017). As well as this, in making a diagnosis of autism clinicians tend to individualise and decontextualise symptoms, mystifying the role of the interaction context of the assessment in producing the behaviour of concern (Turowetz, 2015).

This discussion has primarily been concerned with the conduct of verbal autistic people. However, an important avenue for developing a conversation analytic understanding of autistic people's social practices is the further investigation of non-verbal autistic people's social interaction. In terms of social interaction and socialisation into particular social methods, verbal and non-verbal autistic children differ. Non-verbal autistic children, by definition, do not have access to a set of semiotic fields, specifically speech. This is not to say that they are limited, as the entire social ecology can be made available as resources for action (Mondada, 2016). But it does mean that in most interactional settings, they will stand out in comparison to a norm of privileging speech. Analyses of non-verbal autistic children would help to understand how different activities can be accomplished without the use of speech and what kinds of activities are more likely to suffer disruptions to progressivity. This has been done in the case of aphasic speakers with very minimal verbal output (Bryan, McIntosh, \& Brown, 1998; Goodwin, 1995) and has shown how with support from 
interlocutors, minimally verbal or non-verbal people can participate in complex social activity.

Psychology has been described as a field which consists of "techniques for the disciplining of human difference" (Rose, 1988, p. 187). Those techniques have primarily relied on the comparison of groups or individuals against group norms produced by through measurement and averaging (Rose, 1988). The very existence of the category of autistic people is the result of comparing people against a produced normal distribution of cognition and/or behaviour and the study of autistic people has further perpetuated this negative image based on comparison to a neurotypical normal (Dindar, Lindblom, \& Kärnä, 2017). This thesis attests to the fact that autistic children achieve social competence in interaction with others. It does this by taking seriously autistic children's behaviour as resources available for the intersubjective accomplishment of action in interaction with their family members. Because this research avoids direct comparison with neurotypical interactants or their practices, it produces a different picture of autistic people as competent interactants in their own right. The analyses show how autistic children are competently able to manage the interactional spaces they find themselves in at home with supportive family members. As is likely the case of families in general, these relationships constitute a membership in the same interactional practices (Garfinkel, \& Sacks, 1970); not necessarily just a shared autistic membership, or just a family membership, but membership in a set of available practices that overlap enough that they can engage interactionally with each other.

Being socialised into the interactional practices of a particular community (or serial collectivity; Chapman, 2020) like a family or the autistic community is similar to being socialised into a culture ${ }^{10}$. Hillary (2002) discusses the notion of cross-neurotype communication and advocates that much of the same considerations that are made when interacting with people from different ethnicities, countries et cetera would help produce smoother interaction if made in reference to interacting across neurotypes (e.g.,

\footnotetext{
${ }^{10}$ It is important to note that while the conceptualisation of autism proposed here is as a socially constructed membership category, I am not saying that it is entirely constructed from nothing. Membership categories are constituted by category-bound predicates (i.e., some activities are understood to be done by certain types of people), and correspondingly membership ascription is made based on these predicates (Sacks, 1974; Watson, 1979). The aim here is to recognise that when we (social participants or analysts) make observations and ascribe categories to we are hardly "carving nature at its joints". Instead we are imposing a moral, categorical order which produces individuals with individual differences, and produces groups of people with similar sets of individual differences, for the purposes of social action. Explanations of the nature and origins of those purported individual differences are also part of this moral work. Autistic people are different from others because everyone is different. How we make sense of that difference is the crux of the matter.
} 
neurotypical/autistic interactions). Rather than understanding another's behaviour in terms of their having a social disorder and thus placing all responsibility for interactional trouble on them, interlocutors can presume a distance between themselves such that they both need to meet each other in the middle (Hillary, 2020; Neurodivergent K, 2011). In the interactions analysed for this research, there was no talk of autism in any of the recordings made. The parents and children mutually socialised themselves into a household culture (Pontecorvo, Fasulo, \& Sterponi, 2001) where the children, despite being diagnosed with Autism Spectrum Disorder, are not commonly treated like their ascribed autistic status is a cause of disruptive, or bad, behaviour.

\section{The Future for Discursive Psychology and Neurodiversity Studies}

This thesis demonstrates the analysis of several interactional phenomena as produced in interactions with autistic children. While these analyses have provided important nuance to a consideration of autism-relevant interactional concerns, there are ways this research could be further extended or developed. This section will describe some suggestions for how the specific analyses conducted here could be extended before addressing some larger concerns and critiques with methodology and engagement of autistic participant researchers.

\section{Possible Analytic Targets}

Regarding the discursive respecification of Theory of Mind, further research could expand on this by looking at other ways that children address their and their interlocutors' relative knowledge about each other and their actions. For example, a study of autistic children's responses to other's accounts for the others' behaviour would elucidate how they treat accounts as explanations or as other activities. Because accounts can be made recognisable in a variety of ways (as mentioned above, via prosody, sequence or explicit marking) this may pose troubles for autistic people in recognising accounts as such, especially when they are not responsive to an account solicitation from the child.

As well as accounts, talk that produces one's own or others' thoughts would be a clear environment for the accomplishment of other-attentiveness. From a discursive psychological perspective, no talk can be understood as actually unproblematically reporting anyone's thoughts, but some actions are accomplished with reference to one's thinking. For example, turn-initial "I don't know" can be used as an epistemic hedge regarding what comes next. (Weatherall, 2011), and structures like "I think" or "I thought" can be used to convey a sense of how some relevant object to the action at hand appears, or appeared, to the speaker in a 
kind of reporting of thoughts (Barnes, \& Moss, 2007). Furthering an analysis of autistic children's use and responses to accounts and analysis of their reference to thoughts in interaction are two ways (indirect and direct) that their concern with others' and their experiences can be studied.

In regards to autistic sensation and touch, a way this work could be further developed is with the comparative analysis of cases of touch avoidance like I've shown here with ones that might more appropriately be considered due to sensory issues. What this would look like in the first instance would be a collection of cases where autistic children provide an account for their touch avoidance on the basis of how it feels. This work would also need to tie in to work that looks at autistic children's touch sensitivity generally in order to address what "doing hyper-/hyposensitive" looks like. Ethnomethodological, conversation analytic, and discursive psychological research into "doing sensation" already exists. For example, Mondada (2019) and Fele (2019) have discussed the practices by which taste and smell, respectively, are interactionally accomplished. As well, research has looked into children's discomfort and pain to understand how these become interactionally available (Jenkins, \& Hepburn, 2015). This research has looked at how children interactionally produce pain and discomfort through lexical formulations of the pain (e.g., "My X hurts."), prosody commonly also seen in emotional upset, recognisable pain-indexing cries (e.g., ouch, ow), and physical action like grimacing. This kind of work could provide the basis for a similar analysis of autistic hyper-/hyposensitivity. This would be significant because autistic people are more likely to live with an elevated prevalence of pain (Whitney, \& Shapiro, 2019) and they reportedly present themselves as in pain differently from neurotypical people and/or neurotypical people may not recognise pain in autistics the same way (Allely, 2013). Being able to recognise pain in autistic people could help reduce pressure on autistic people struggling to make their pain recognisable to neurotypical people.

\section{Cognition, Behaviour, and Masking}

None of the children involved in this research were formally assessed (by myself) for their Theory of Mind capacities, touch sensitivity or any other formal psychological/psychiatric feature. The children were selected for participation on the basis of having received a formal diagnosis of Autism Spectrum Disorder in the past. This was because the aim of analysing these children's social interactions was to show what children who have received such a diagnosis interact despite it, and how their social interaction can be studied in a naturalistic, emic way. There is no principled way of claiming that any individual 
act was committed as a result of their autism so that concern was bracketed off from the analysis. But a possible critique that could be made is that while all these children received diagnoses, autism is reportedly highly heterogenous (Masi, DeMayo, Glozier, \& Guastella, 2017) and as such these children may just have been particularly socially capable or were compensating for their putative deficits through alternative cognitive strategies.

Compensation refers to "the processes contributing to improved behavioural presentation of a neurodevelopmental disorder, despite persisting core deficit(s) at cognitive and/or neurobiological levels" (Livingston, \& Happé, 2017, p. 731). Examples of compensatory strategies include using prepared scripts in social interaction or reasoning about others' mental states by comparing their conduct to book/movie characters (Livingston, Shah, Milner, \& Happé, 2020). Compensation is said to explain autistic people's ability to act in socially competent ways despite formal assessments suggesting the presence of Theory of Mind deficits that would hinder that capacity (Livingston, Colvert, Social Relationships Study Team, Bolton, \& Happé, 2019). As well autistic people themselves report that engaging in neurotypically dominant activities or ways of thinking can be cognitively/emotionally taxing (Livingston, Shah, \& Happé, 2019; Crompton, Hallett, Ropar, Flynn, \& Fletcher-Watson, 2020).

A complete respecification of compensation is beyond the aims and scope of this thesis but some comments are made here to address why compensation is a problematic notion within a discursive psychological study of autism. In much the same way this thesis critiques the expectations one should have of individuals based on their autism diagnosis, it is important to critique the notion of a discrepancy between cognitive ability formally assessed in standardised tests of social cognition and the actual behaviour that cognitive ability is said to govern. Part of the notion of compensation seems to be prioritising the formal cognitive assessments over the actual behaviour in determining whether a "core deficit" is present. From an ethnomethodological/conversation analytic perspective, cognitive assessments are themselves interactional accomplishments (Maynard, \& Turowetz, 2017); as they are built in response to the local contingencies the relationship between experimental findings about some behaviour and that behaviour in situ is complicated. Understanding a behaviour in one interactional setting does not necessarily provide an understanding of that behaviour elsewhere. As such, prioritising the results of formal cognitive assessments over observations of behaviour in ordinary social life is not warranted. What is important to this thesis and the analyses it reports is that intersubjectivity in social interaction between autistic children and 
their family members is accomplished and that accomplishment is produced through and made visible in sophisticated practical actions.

As a direction for future discursive psychological work, analysts could investigate compensation as psychological notion that is interactionally realised. This could approach the notion of compensation in a variety of different ways. One way would be to look at the ways that autistic people characterise their action-in-progress as difficult for them for reasons associated with their neurodivergence. For example, an analyst may analyse cases of an autistic speaker accounting for an inference made of an interlocutor by describing their inference as relying on learned psychological theory or characters from a book. Alternatively, analysts could study moments where participants characterise behaviour as demonstrative of compensation failing, accounting for their behaviour as the result of struggling against neurotypical expectations, or just displays of emotionality and discomfort in settings that autistic people report being difficult.

\section{Autistic Participation in Research}

A primary aim of this thesis, and of critical autism studies generally, is to challenge the narratives and practices that marginalise autistic people (Woods, Arnold, Milton, \& Graby, 2018). Analysing autistic people's social interaction as it happens and attending to the same issues that they did is a way of challenging the common practice in psychology of pitting autistic against non-autistic social skills in a test against neurotypical expectations. As such it constitutes a step towards the emancipatory goal of critical autism studies. A further step (and to many, a requisite one; Oliver, 1997; Chown, et al., 2017) is to involve autistic people themselves in the direction of the research and the analysis. This is not only for ethical reasons but because it would also support the validity of the analysis.

The ethical side of this critique is the idea that without autistic voices in a research project on autism, research can be directed in ways that do not serve the interests of the autistic participants and autistic community (Milton, \& Bracher, 2013). In fact, that has been the case for much research on autism and autistic people. Examples of this include the funding focus on research into the neuro-genetic development, and treatment of, autistic features (Pellicano, Dinsmore, \& Charman, 2014), and the focus of psychological research into aspects of autism as they appear to others (e.g., social "disability") and not aspects of autism as they are experienced by autistic people (e.g., embodiment and sensoriality; Fournier et al., 2010; Leary \& Hill, 1996). This balance in the power to define autism 
prioritises the researchers' interests and not the autistic participants'/community's (FletcherWatson et al., 2018), perpetuating a vision of autism in comparison to the comparatively unscrutinised neurotypical contribution to the difficulties in the lived experiences of autism.

Future conversation analytic research into autistic people's social interaction should involve autistic stakeholders in design and analysis. Doing so benefits the study of autistic social interaction and of talk in general. Including autistic people on the research team helps to direct research such that it can meet the needs or interests of the autistic participants and/or community. Furthermore, autistic participants or community members may have insights into the methods and practices used by other autistic people, or their interests and social goals. Like in national/ethnic cultures, autistic people may make sense of their own and other's behaviours in different ways. Examples of this include the way silences of different lengths are treated more or less problematically across speaking communities (e.g., the Garrwa language; Gardner, \& Mushin, 2015), or the variability of normative phone call opening sequences across national cultures (Schegloff, 1968; Schegloff, 1979; Houtkoop-Steenstra, 1991; Lindström, 1994). Having some cultural competence in the relevant cultures makes more accessible the differences between cultures being documented in the above cited studies.

Despite the utility of the next turn proof procedure for analysing how participants understand their local interaction context, discursive psychological analysis still relies on analysts describing participants' treatment of action as it appears to them (Potter, Edwards, \& Wetherell, 1993). For analysts to understand what participants are doing, analysts still have to describe actions. Because actions are not specific, delineated phenomena so easily categorised (Enfield, \& Sidnell, 2017), the analyst's decision to describe interactional moves, and their responses, in certain ways in effect leads to the analysts understanding the accountability of certain moves in ways potentially quite different from participants themselves. In this way, autistic participant researchers could contribute to the analysis by providing descriptions of action that may not necessarily be easily available to the nonautistic analysts. An example of this in autistic interaction might be the ways eye gaze of other facial expressions are used. Experimental research and autobiographical reports show that autistic people may de-prioritise language in social interaction (Hillary, 2020), and understand/use interactional resources like eye gaze and expression, and movement differently from neurotypical people (Fletcher-Watson, Leekam, Benson, Frank, \& Findlay, 2009; Davidson, \& Smith, 2009). Rather than just pointing out that they do not use these 
interactional resources in the same ways as neurotypical people, autistic analysts might be more able to describe alternative ways autistic people manage speaker selection and affective stances. Conversation analytic research has the benefit of the next turn proof procedure and a concern with participant's orientations that allows analysts to make sense of action with reference to how participants make sense of it, yet analysis can benefit from some degree of understanding members' practices to recognise (i.e., describe) what might be otherwise foreign normative cultural practices.

While conversation analytic research is highly technical, conversation analysts have used participatory frameworks to design research, select data excerpts for transcription and analysis and analyse the data in collaboration with untrained participants. Most relevantly this has been done with participants with neurological diagnoses, such as intellectual disabilities (Williams, 2011) and dementia (Williams, Webb, Read, James, \& Davis, 2020). For example, Williams (2011) describes how they facilitated a team of patients with early onset dementia to engage with all phases of conversation analytic research. Excepts were chosen by the patient researchers for transcription and analysis, and these were fed back to the group by academics who developed their analyses on the basis of the patient researchers' contributions. In this way, the project was directed by them and supported by their members' knowledge.

In my research, participant involvement was primarily actioned by inviting families to choose for themselves how, when, where, and what they would record for analysis. In discussions with the families, we talked about the kinds of things that were of interest to us and what kinds of things might have come up in the recording. In future research, I would like (and I would encourage others) to more formally invite participants and relevant community members to participate in the design and analysis of data including them.

\section{Applications}

Understanding autism in the way this thesis has exemplified has important practical implications, primarily for parents/caregivers and others who routinely engage with autistic people in support or service roles. Interventions are commonly designed to develop autistic children's social skills by training Theory of Mind, however reviews of the Theory of Mind intervention literature shows that improvements on Theory of Mind tasks are not likely to generalise to other contexts or are not maintained over time, and it is uncertain if these skill increases even impact the quality of real life social interactions (Fletcher-Watson, McConnell, Manola, \& McConachie, 2014; Hadwin \& Kovshoff, 2013). The analyses 
reported in this thesis support the idea that an appropriate avenue for intervention design might instead involve teaching an interactional approach focused on the mutual production of action in context.

Instead of focusing on training autistic individuals' interactional skills, interventions could focus on teaching both autistic people and their friends, family, and/or professionals about how people make themselves understood in interaction. In this way, people who interact with autistic people might learn to appreciate autistic people's conduct for what it accomplishes rather than for what it appears to lack. An educational approach to social interactional intervention has been developed called the conversation analytic role-play method, or CARM (Stokoe, 2014). With CARM, people are shown cases of their own reallife interactions where they are trying to accomplish some activity and are shown how, across a collection of such instances, some interactional moves work to accomplish that activity while others do not. This has been used successfully by teachers in early childhood education in resolution of children's disputes (Church \& Bateman, 2019). A CARM-like approach may help family, friends, and professionals to, at least, mitigate some degree of the interactional difficulty associated with autism by demonstrating their part in the double empathy problem of interaction (Milton, 2012) and increasing their own sensitivity to autistic children's interactional moves. An example of this kind of increasing sensitivity is discussed in relation to reflective interventionist conversation analysis (O’Reilly, Kiyimba, Lester, \& Muskett, 2020), an approach to applied conversation analysis that encourages looking at interactions in the provision of clinical interventions in order that best practices for accomplishing some intervention-relevant goal can be identified.

\section{Conclusion}

Since its description by Leo Kanner and Hans Asperger in the 1940s, autism has been characterised as a condition which impacts children's development of appropriate social cognition and behaviour. This thesis, however, demonstrates that autistic children engage a variety of sophisticated social practices in interaction with others. Those practices contribute to the intersubjectivity accomplished between them and their interlocutors. By their deployment of such practices, the children interactionally accomplished complex relationships with their family members, attended to the ways their action was, or could have been, treated by interlocutors, and actively participated in the constitution of everyday homelife. 
Whether someone is autistic, or not, is an interactional accomplishment. The findings reported here highlight how an autism diagnosis is not necessarily required for understanding how or why any so-diagnosed child is acting in any moment. In the data, neither parents nor children needed to draw on autism or its commonly recognised predicates to make sense of each other's or their own action. Instead, local conduct was organised with respect to local organisation. The children made themselves understood utilising the design and local organisation of their action. They demonstrated sensitivity to what their family members did and how their own conduct was recognised.

While autism research has always highlighted cognition, a recent trend is to consider the autistic mind in terms of how it is embodied. Recognising that autistic people report different sensory experiences to neurotypical people is a good step in resituating autistic people in the social world. This thesis goes further to show how moments of sensation are achieved within the course of action and how they are avoided for other activities. These analyses show how, in the moment, the children accomplish a kind of embodied competence, using their bodies as social resources as much as their talk to negotiate the complex landscape of parent/child authority and autonomy. In these cases, the autistic mind is clearly accomplished in embodiment as actions of the body interactionally accomplish decision making and authority, affection, planning and other commonly mentalised psychological notions.

How we as researchers, and as members of society, understand autism depends on the analytic choices we make when attempting to understand behaviour. This thesis highlights what is gained by treating autistic people as social agents in their own right. The parents analysed here demonstrated a sensitivity towards their children that would be an ideal model for everyone in engaging with autistic people. Neurotypical people's sensitivities to action and the available interactional resources, and trust that one's interlocutor is acting agentically, contribute to the social ecology in which autistic people can thrive. 


\section{References}

Adamson, L. B., McArthur, D., Markov, Y., Dunbar, B., \& Bakeman, R. (2001). Autism and joint attention: Young children's responses to maternal bids. Journal of Applied Developmental Psychology, 22(4), 439-453. https://doi.org/10.1016/S01933973(01)00089-2

Alkhaldi, R. S., Sheppard, E., \& Mitchell, P. (2019). Is there a link between autistic people being perceived unfavorably and having a mind that Is difficult to read? Journal of Autism and Developmental Disorders, 49(10), 3973-3982. https://doi.org/10.1007/s10803-019-04101-1

Allely, C. S. (2013). Pain sensitivity and observer perception of pain in individuals with autistic spectrum disorder. The Scientific World Journal, 2013, 1-20. https://doi.org/10.1155/2013/916178

American Psychiatric Association (Ed.). (2013). Diagnostic and statistical manual of mental disorders: DSM-5 (5th ed). American Psychiatric Association.

Anderson, D. K., Lord, C., Risi, S., DiLavore, P. S., Shulman, C., Thurm, A., Welch, K., \& Pickles, A. (2007). Patterns of growth in verbal abilities among children with autism spectrum disorder. Journal of Consulting and Clinical Psychology, 75(4), 594-604. https://doi.org/10.1037/0022-006X.75.4.594

Antaki, C. (1994). Explaining and arguing: The social organization of accounts. Sage Publications.

Antaki, C. (1996). Explanation slots as resources in interaction. British Journal of Social Psychology, 35(3), 415-432. https://doi.org/10.1111/j.2044-8309.1996.tb01105.x

Antaki, C. (2004). Reading minds or dealing with interactional implications? Theory \& Psychology, 14(5), 667-683. https://doi.org/10.1177/0959354304046178

Arciuli, J. (2014). Prosody and autism. In J. Arciuli \& J. Brock (Eds.), Communication in Autism (pp. 103-124). John Benjamins Publishing Company.

Aronsson, K., \& Cekaite, A. (2011). Activity contracts and directives in everyday family politics. Discourse \& Society, 22(2), 137-154.

https://doi.org/10.1177/0957926510392124

Baranek, G. T. (1999). Autism during infancy: A retrospective video analysis of sensorymotor and social behaviors at 9-12 months of age. Journal of Autism and Developmental Disorders, 29(3), 213-224. https://doi.org/10.1023/A:1023080005650

Baranek, G. T., David, F. J., Poe, M. D., Stone, W. L., \& Watson, L. R. (2006). Sensory experiences questionnaire: Discriminating sensory features in young children with 
autism, developmental delays, and typical development: SEQ. Journal of Child Psychology and Psychiatry, 47(6), 591-601. https://doi.org/10.1111/j.14697610.2005.01546.x

Barker, R. G., Wright, H. F., \& Barker, L. S. (1961). One boy's day: A specimen record of behavior. Archon Books.

Barnes, R., \& Moss, D. (2007). Communicating a feeling: The social organization of 'private thoughts'. Discourse Studies, 9(2), 123-148. https://doi.org/10.1177/1461445607075339

Baron-Cohen, S. (2004). The cognitive neuroscience of autism. Journal of Neurology, Neurosurgery \& Psychiatry, 75(7), 945-948. https://doi.org/10.1136/jnnp.2003.018713

Baron-Cohen, S., Jolliffe, T., Mortimore, C., \& Robertson, M. (1997). Another advanced test of theory of mind: Evidence from very high functioning adults with autism or asperger syndrome. Journal of Child Psychology and Psychiatry, and Allied Disciplines, 38(7), 813-822.

Baron-Cohen, S., Leslie, A. M., \& Frith, U. (1985). Does the autistic child have a "theory of mind"? Cognition, 21(1), 37-46.

Baron-Cohen, Simon. (1991a). Do people with autism understand what causes emotion? Child Development, 62(2), 385-395. JSTOR. https://doi.org/10.2307/1131011

Baron-Cohen, Simon. (1991b). Precursors to a theory of mind: Understanding attention in others. In A. Whiten (Ed.), Natural theories of mind: Evolution, development, and simulation of everyday mindreading (pp. 233-251).

Baron-Cohen, Simon. (1997). Mindblindness: An essay on autism and theory of mind. MIT.

Barriage, S., \& Searles, D. K. (2019). "Okay okay okay, now the video is on”: An analysis of young children's orientations to the video camera in recordings of family interactions. Journal of Childhood Studies, 18-41. https://doi.org/10.18357/jcs00019172

Bartolucci, G., Pierce, S., Streiner, D., \& Eppel, P. T. (1976). Phonological investigation of verbal autistic and mentally retarded subjects. Journal of Autism and Childhood Schizophrenia, 6(4), 303-316. https://doi.org/10.1007/BF01537908

Bateman, A. (2017). Hearing children's voices through a conversation analysis approach. International Journal of Early Years Education, 25(3), 241-256. https://doi.org/10.1080/09669760.2017.1344624

Baumeister, R. F., Vohs, K. D., \& Funder, D. C. (2007). Psychology as the science of selfreports and finger movements: Whatever happened to actual behavior? Perspectives on 
Psychological Science, 2(4), 396-403. https://doi.org/10.1111/j.1745$\underline{6916.2007 .00051 . x}$

Ben-Sasson, A., Soto, T. W., Martínez-Pedraza, F., \& Carter, A. S. (2013). Early sensory over-responsivity in toddlers with autism spectrum disorders as a predictor of family impairment and parenting stress: Sensory over-responsivity, family impairment, and autism. Journal of Child Psychology and Psychiatry, 54(8), 846-853. https://doi.org/10.1111/jcpp.12035

Bertilsdotter Rosqvist, H., Chown, N., \& Stenning, A. (2020). Neurodiversity studies: A new critical paradigm. https://www.taylorfrancis.com/books/9780429322297

Bertilsdotter Rosqvist, H., Stenning, A., \& Chown, N. (2020). Neurodiversity Studies: Proposing a new field of inquiry. In Neurodiversity Studies: A new critical paradigm (pp. 226-229). https://www.taylorfrancis.com/books/9780429322297

Blakemore, S.-J., Tavassoli, T., Calò, S., Thomas, R. M., Catmur, C., Frith, U., \& Haggard, P. (2006). Tactile sensitivity in Asperger syndrome. Brain and Cognition, 61(1), 5-13. https://doi.org/10.1016/j.bandc.2005.12.013

Blum-Kulka, S. (1997). Dinner talk: Cultural patterns of sociability and socialization in family discourse. L. Erlbaum Assoc. Publishers.

Bögels, S., \& Levinson, S. C. (2017). The brain behind the response: Insights into turn-taking in conversation from neuroimaging. Research on Language and Social Interaction, 50(1), 71-89. https://doi.org/10.1080/08351813.2017.1262118

Bottema-Beutel, K. (2017). Glimpses into the blind spot: Social interaction and autism. Journal of Communication Disorders, 68, 24-34. https://doi.org/10.1016/j.jcomdis.2017.06.008

Bottema-Beutel, K., Kim, S. Y., \& Crowley, S. (2019). A systematic review and metaregression analysis of social functioning correlates in autism and typical development: Bottema-Beutel et al./Social functioning meta-analysis. Autism Research, 12(2), 152175. https://doi.org/10.1002/aur.2055

Bottema-Beutel, K., Louick, R., \& White, R. (2015). Repetition, response mobilization, and face: Analysis of group interactions with a 19-year-old with Asperger syndrome. Journal of Communication Disorders, 58, 179-193. https://doi.org/10.1016/j.jcomdis.2015.05.002

Boucher, J. (2012). Putting theory of mind in its place: Psychological explanations of the socio-emotional-communicative impairments in autistic spectrum disorder. Autism, 16(3), 226-246. https://doi.org/10.1177/1362361311430403 
Brown, P., \& Levinson, S. C. (1987). Politeness: Some universals in language usage. Cambridge University Press.

Bryan, K., McIntosh, J., \& Brown, D. (1998). Extending conversation analysis to nonverbal communication. Aphasiology, 12(2), 179-188. https://doi.org/10.1080/02687039808250474

Burgoon, J. K. (1991). Relational message interpretations of touch, conversational distance, and posture. Journal of Nonverbal Behavior, 15(4), 233-259. https://doi.org/10.1007/BF00986924

Butler, C. W. (2008). Talk and social interaction in the playground. Ashgate.

Cascio, C., McGlone, F., Folger, S., Tannan, V., Baranek, G., Pelphrey, K. A., \& Essick, G. (2008). Tactile perception in adults with autism: A multidimensional psychophysical study. Journal of Autism and Developmental Disorders, 38(1), 127-137. https://doi.org/10.1007/s10803-007-0370-8

Cekaite, A. (2010). Shepherding the child: Embodied directive sequences in parent-child interactions. Text \& Talk - An Interdisciplinary Journal of Language, Discourse \& Communication Studies, 30(1), 1-25. https://doi.org/10.1515/text.2010.001

Cekaite, A. (2016). Touch as social control: Haptic organization of attention in adult-child interactions. Journal of Pragmatics, 92, 30-42. https://doi.org/10.1016/j.pragma.2015.11.003

Cekaite, A., \& Bergnehr, D. (2018). Affectionate touch and care: Embodied intimacy, compassion and control in early childhood education. European Early Childhood Education Research Journal, 26(6), 940-955. https://doi.org/10.1080/1350293X.2018.1533710

Cekaite, A., \& Kvist Holm, M. (2017). The comforting touch: Tactile intimacy and talk in managing children's distress. Research on Language and Social Interaction, 50(2), 109-127. https://doi.org/10.1080/08351813.2017.1301293

Chapman, R. (In Press). Neurodiversity and the social ecology of mental functions. Perspectives on Psychological Science.

Chapman, R. (2019). Neurodiversity theory and its discontents: Autism, schizophrenia, and the social model of disability. In R. Bluhm (Ed.), The Bloomsbury Companion to Philosophy of Psychiatry (pp. 371-390). Bloomsbury Academic.

https://doi.org/10.5040/9781350024090 
Chapman, R. (2020). The reality of autism: On the metaphysics of disorder and diversity. Philosophical Psychology, 33(6), 799-819. https://doi.org/10.1080/09515089.2020.1751103

Chomsky, N. (1957). Syntactic structures. (p. 116). Mouton.

Chown, N., \& Beardon, L. (2017). Autism theory. In F. R. Volkmar (Ed.), Encyclopedia of Autism Spectrum Disorders (pp. 1-7). Springer New York. https://doi.org/10.1007/978$\underline{1-4614-6435-8 \_102224-1}$

Chown, N., Robinson, J., Beardon, L., Downing, J., Hughes, L., Leatherland, J., Fox, K., Hickman, L., \& MacGregor, D. (2017). Improving research about us, with us: A draft framework for inclusive autism research. Disability \& Society, 32(5), 720-734. https://doi.org/10.1080/09687599.2017.1320273

Church, A. (2009). Preference organisation and peer disputes: How young children resolve conflict (1st ed.). Routledge. https://doi.org/10.4324/9781315602035

Church, A., \& Bateman, A. (2019). Methodology and professional development: Conversation Analytic Role-play Method (CARM) for early childhood education. Journal of Pragmatics, 143, 242-254. https://doi.org/10.1016/j.pragma.2019.01.022

Clayman, S. E. (2012). Turn-constructional units and the transition-relevance place. In J. Sidnell \& T. Stivers (Eds.), The Handbook of Conversation Analysis (pp. 151-166). John Wiley \& Sons, Ltd. https://doi.org/10.1002/9781118325001.ch8

Clayman, S. E., \& Heritage, J. C. (2014). Benefactors and beneficiaries: Benefactive status and stance in the management of offers and requests. In P. Drew \& E. Couper-Kuhlen (Eds.), Studies in Language and Social Interaction (Vol. 26, pp. 55-86). John Benjamins Publishing Company. https://doi.org/10.1075/slsi.26.03cla

Cleland, J., Gibbon, F. E., Peppé, S. J. E., O’Hare, A., \& Rutherford, M. (2010). Phonetic and phonological errors in children with high functioning autism and Asperger syndrome. International Journal of Speech-Language Pathology, 12(1), 69-76. https://doi.org/10.3109/17549500903469980

Clifford, S. M., Hudry, K., Elsabbagh, M., Charman, T., Johnson, M. H., \& The BASIS Team. (2013). Temperament in the first 2 years of life in infants at high-risk for autism spectrum disorders. Journal of Autism and Developmental Disorders, 43(3), 673-686. https://doi.org/10.1007/s10803-012-1612-y

Clift, R. (2001). Meaning in interaction: The case of actually. Language, 77(2), 245-291. JSTOR. 
Cook Jr, E., Courchesne, R., Lord, C., Cox, N. J., Yan, S., Lincoln, A., Haas, R., Courchesne, E., \& Leventhal, B. L. (1997). Evidence of linkage between the serotonin transporter and autistic disorder. Molecular Psychiatry, 2(3), 247-250. https://doi.org/10.1038/sj.mp.4000266

Coulter, J. (1979). The social construction of mind: Studies in ethnomethodology and linguistic philosophy. Palgrave Macmillan Limited. https://public.ebookcentral.proquest.com/choice/publicfullrecord.aspx?p=5643927

Coulter, J. (2008). Twenty-five theses against cognitivism. Theory, Culture \& Society, 25(2), 19-32. https://doi.org/10.1177/0263276407086789

Couper-Kuhlen, E. (2012). Turn continuation and clause combinations. Discourse Processes, 49(3-4), 273-299. https://doi.org/10.1080/0163853X.2012.664111

Crane, L., Chester, J. W., Goddard, L., Henry, L. A., \& Hill, E. (2016). Experiences of autism diagnosis: A survey of over 1000 parents in the United Kingdom. Autism, 20(2), 153162. https://doi.org/10.1177/1362361315573636

Craven, A., \& Potter, J. (2010). Directives: Entitlement and contingency in action. Discourse Studies, 12(4), 419-442. https://doi.org/10.1177/1461445610370126

Crompton, C. J., Hallett, S., Ropar, D., Flynn, E., \& Fletcher-Watson, S. (2020). 'I never realised everybody felt as happy as I do when I am around autistic people': A thematic analysis of autistic adults' relationships with autistic and neurotypical friends and family: Autism, 1438-1448. https://doi.org/10.1177/1362361320908976

Cullen, L., \& Barlow, J. (2002). 'Kiss, Cuddle, Squeeze': The experiences and meaning of touch among parents of children with autism attending a touch therapy programme. Journal of Child Health Care, 6(3), 171-181. https://doi.org/10.1177/136749350200600303

Curl, T. S., \& Drew, P. (2008). Contingency and action: A comparison of two forms of requesting. Research on Language \& Social Interaction, 41(2), 129-153. https://doi.org/10.1080/08351810802028613

Damico, J. S., \& Nelson, R. L. (2005). Interpreting problematic behavior: Systematic compensatory adaptations as emergent phenomena in autism. Clinical Linguistics \& Phonetics, 19(5), 405-417. https://doi.org/10.1080/02699200400027163

Davidson, J., \& Smith, M. (2009). Autistic autobiographies and more-than-human emotional geographies. Environment and Planning D: Society and Space, 27(5), 898-916. https://doi.org/10.1068/d4308 
Demetriou, E. A., Lampit, A., Quintana, D. S., Naismith, S. L., Song, Y. J. C., Pye, J. E., Hickie, I., \& Guastella, A. J. (2018). Autism spectrum disorders: A meta-analysis of executive function. Molecular Psychiatry, 23(5), 1198-1204. https://doi.org/10.1038/mp.2017.75

Demuth, C. (2013). Handling power-asymmetry in interactions with infants: A comparative socio-cultural perspective. Interaction Studies, 14(2), 212-239. https://doi.org/10.1075/is.14.2.04dem

den Houting, J., \& Pellicano, E. (2019). A portfolio analysis of autism research funding in Australia, 2008-2017. Journal of Autism and Developmental Disorders, 49(11), 44004408. https://doi.org/10.1007/s10803-019-04155-1

Deppermann, A. (2012). How does 'cognition' matter to the analysis of talk-in-interaction? Language Sciences, 34(6), 746-767. https://doi.org/10.1016/j.langsci.2012.04.013 de Ruiter, J. P., \& Albert, S. (2017). An appeal for a methodological fusion of conversation analysis and experimental psychology. Research on Language and Social Interaction, 50(1), 90-107. https://doi.org/10.1080/08351813.2017.1262050

Dichter, G. S. (2012). Functional magnetic resonance imaging of autism spectrum disorders. Dialogues in Clinical Neuroscience, 14(3), 319-351.

Diehl, J. J., Bennetto, L., Watson, D., Gunlogson, C., \& McDonough, J. (2008). Resolving ambiguity: A psycholinguistic approach to understanding prosody processing in highfunctioning autism. Brain and Language, 106(2), 144-152. https://doi.org/10.1016/j.bandl.2008.04.002

Di Martino, A., Yan, C.-G., Li, Q., Denio, E., Castellanos, F. X., Alaerts, K., Anderson, J. S., Assaf, M., Bookheimer, S. Y., Dapretto, M., Deen, B., Delmonte, S., Dinstein, I., ErtlWagner, B., Fair, D. A., Gallagher, L., Kennedy, D. P., Keown, C. L., Keysers, C., ... Milham, M. P. (2014). The autism brain imaging data exchange: Towards a large-scale evaluation of the intrinsic brain architecture in autism. Molecular Psychiatry, 19(6), 659-667. https://doi.org/10.1038/mp.2013.78

Dindar, K., Korkiakangas, T., Laitila, A., \& Kärnä, E. (2016a). Facilitating joint attention with salient pointing in interactions involving children with autism spectrum disorder. Gesture, 15(3), 372-403. https://doi.org/10.1075/gest.15.3.06din

Dindar, K., Korkiakangas, T., Laitila, A., \& Kärnä, E. (2016b). Building mutual mnderstanding: How children with autism spectrum disorder manage interactional trouble. Journal of Interactional Research in Communication Disorders, 7(1), 49-77. https://doi.org/10.1558/jircd.v7i1.28228 
Dindar, K., Lindblom, A., \& Kärnä, E. (2017). The construction of communicative (in)competence in autism: A focus on methodological decisions. Disability \& Society, 32(6), 868-891. https://doi.org/10.1080/09687599.2017.1329709

Dinishak, J. (2016). The deficit view and its critics. Disability Studies Quarterly, 36(4). https://doi.org/10.18061/dsq.v36i4.5236

Duranti, A. (1997). Linguistic Anthropology (1st ed.). Cambridge University Press. https://doi.org/10.1017/CBO9780511810190

Edwards, D. (1997). Discourse and Cognition. Sage Publications.

Edwards, D. (2012). Discursive and scientific psychology: Discursive and scientific psychology. British Journal of Social Psychology, 51(3), 425-435. https://doi.org/10.1111/j.2044-8309.2012.02103.x

Edwards, D., \& Potter, J. (1992). Discursive psychology. Sage Publications.

Edwards, D., \& Potter, J. (2005). Discursive psychology, mental states and descriptions. In H. te Molder \& J. Potter (Eds.), Conversation and Cognition (pp. 241-259). Cambridge University Press; Cambridge Core. https://doi.org/10.1017/CBO9780511489990.012

Enfield, N., \& Sidnell, J. (2017). On the concept of action in the study of interaction. Discourse Studies, 19(5), 515-535. https://doi.org/10.1177/1461445617730235

Ervin-Tripp, S. (1976). Is Sybil there? The structure of some American English directives. Language in Society, 5(1), 25-66. https://doi.org/10.1017/S0047404500006849

Eyal, G. (2013). For a sociology of expertise: The social origins of the autism epidemic. American Journal of Sociology, 118(4), 863-907. https://doi.org/10.1086/668448

Fele, G. (2019). Olfactory objects: Recognizing, describing and assessing smells during professional tasting sessions. In D. Day \& J. Wagner (Eds.), Objects, Bodies and Work Practice (Issue Vol. 3, pp. 250-284). Multilingual Matters; eBook Collection (EBSCOhost).

http://helicon.vuw.ac.nz/login?url=http://search.ebscohost.com/login.aspx?direct=true\& $\underline{\mathrm{db}=\text { nlebk \&AN}=2182710 \& \text { site }=\text { ehost-live }}$

Fleer, M., \& Hedegaard, M. (2010). Children's development as participation in everyday practices across different institutions. Mind, Culture, and Activity, 17(2), 149-168. https://doi.org/10.1080/10749030903222760

Fletcher-Watson, S., Leekam, S. R., Benson, V., Frank, M. C., \& Findlay, J. M. (2009). Eyemovements reveal attention to social information in autism spectrum disorder. Neuropsychologia, 47(1), 248-257. https://doi.org/10.1016/j.neuropsychologia.2008.07.016 
Fletcher-Watson, Sue, Adams, J., Brook, K., Charman, T., Crane, L., Cusack, J., Leekam, S., Milton, D., Parr, J. R., \& Pellicano, E. (2019). Making the future together: Shaping autism research through meaningful participation. Autism, 23(4), 943-953. https://doi.org/10.1177/1362361318786721

Fletcher-Watson, Sue, McConnell, F., Manola, E., \& McConachie, H. (2014). Interventions based on the Theory of Mind cognitive model for autism spectrum disorder (ASD). Cochrane Database of Systematic Reviews. https://doi.org/10.1002/14651858.CD008785.pub2

Foss-Feig, J. H., Heacock, J. L., \& Cascio, C. J. (2012). Tactile responsiveness patterns and their association with core features in autism spectrum disorders. Research in Autism Spectrum Disorders, 6(1), 337-344. https://doi.org/10.1016/j.rasd.2011.06.007

Foucault, M. (1984). The history of sexuality. Penguin.

Foucault, M. (1991). Discipline and punish: The birth of the prison. Penguin Books.

Fournier, K. A., Hass, C. J., Naik, S. K., Lodha, N., \& Cauraugh, J. H. (2010). Motor coordination in autism spectrum disorders: A synthesis and meta-analysis. Journal of Autism and Developmental Disorders, 40(10), 1227-1240. https://doi.org/10.1007/s10803-010-0981-3

Francis, D., \& Hester, S. (2004). An invitation to ethnomethodology: Language, society and social interaction. SAGE Publications Ltd. https://doi.org/10.4135/9781849208567

Frankel, R. (1982). Autism for all practical purposes: A micro-interactional view. Topics in Language Disorders, 3(1), 33-42.

Frith, U. (Ed.). (1991). Autism and Asperger syndrome. Cambridge University Press.

Frith, U., \& de Vignemont, F. (2005). Egocentrism, allocentrism, and Asperger syndrome. Consciousness and Cognition, 14(4), 719-738. https://doi.org/10.1016/j.concog.2005.04.006

Gallagher, S. (2004). Understanding interpersonal problems in autism: Interaction theory as an alternative to theory of mind. Philosophy, Psychiatry, \&amp; Psychology, 11(3), 199-217. https://doi.org/10.1353/ppp.2004.0063

Gallagher, S. (2020). Action and interaction (1st ed.). Oxford University Press. https://doi.org/10.1093/oso/9780198846345.001.0001

Gardner, R., \& Mushin, I. (2015). Expanded transition spaces: The case of Garrwa. Frontiers in Psychology, 6, 251. https://doi.org/10.3389/fpsyg.2015.00251 
Garfinkel, H. (1963). A conception of, and experiments with, "trust" as a condition of stable concerted actions. In O. J. Harvey (Ed.), Motivation and Social Interaction (pp. 187238). Ronald Press.

Garfinkel, H. (1967). Studies in ethnomethodology. Prentice-Hall.

Garfinkel, H., \& Rawls, A. W. (2002). Ethnomethodology's program: Working out Durkeim's aphorism. Rowman \& Littlefield Publishers.

Garfinkel, H., \& Sacks, H. (1970). On formal structures of practical actions. In J. Mickinney \& E. A. Tiryakian (Eds.), Theoretical Sociology: Perspectives and developments (pp. 337-366). Appleton-Century-Crofts.

Gernsbacher, M. A., Dawson, M., \& Hill Goldsmith, H. (2005). Three reasons not to believe in an autism epidemic. Current Directions in Psychological Science, 14(2), 55-58. https://doi.org/10.1111/j.0963-7214.2005.00334.x

Gernsbacher, M. A., \& Yergeau, M. (2019). Empirical failures of the claim that Autistic people lack a theory of mind. Archives of Scientific Psychology, 7(1), 102-118. https://doi.org/10.1037/arc0000067

Geschwind, D. H. (2011). Genetics of autism spectrum disorders. Trends in Cognitive Sciences, 15(9), 409-416. https://doi.org/10.1016/j.tics.2011.07.003

Goffman, E. (1990). The presentation of self in everyday life (Repr). Penguin.

Goodley, D. (2016). Disability studies: An interdisciplinary introduction (2nd edition). Sage Ltd.

Goodwin, C. (1995). Co-constructing meaning in conversations with an aphasic man. Research on Language and Social Interaction, 28(3), 233-260. https://doi.org/10.1207/s15327973rlsi2803_4

Goodwin, C. (2000). Action and embodiment within situated human interaction. Journal of Pragmatics, 32(10), 1489-1522. https://doi.org/10.1016/S0378-2166(99)00096-X

Goodwin, C. (2013). The co-operative, transformative organization of human action and knowledge. Journal of Pragmatics, 46(1), 8-23. https://doi.org/10.1016/j.pragma.2012.09.003

Goodwin, C., \& Heritage, J. (1990). Conversation analysis. Annual Review of Anthropology, 19(1), 283-307. https://doi.org/10.1146/annurev.an.19.100190.001435

Goodwin, M. H., \& Cekaite, A. (2018). Embodied family choreography. Routledge.

Goodwin, Marjorie Harness. (2006). Participation, affect, and trajectory in family directive/response sequences. Text \& Talk - An Interdisciplinary Journal of Language, 
Discourse Communication Studies, 26(4-5), 515-543.

https://doi.org/10.1515/TEXT.2006.021

Goodwin, Marjorie Harness. (2017). Haptic sociality. In Intercorporeality (Vol. 1). Oxford University Press. https://doi.org/10.1093/acprof:oso/9780190210465.003.0004

Goodwin, Marjorie Harness. (2020). The interactive construction of a hug sequence. In A. Cekaite \& L. Mondada (Eds.), Touch in social interaction: Touch, language and body (pp. 27-53). Routledge.

Grandin, T. (1992). Calming effects of deep touch pressure in patients with autistic disorder, college students, and animals. Journal of Child and Adolescent Psychopharmacology, 2(1), 63-72. https://doi.org/10.1089/cap.1992.2.63

Hacking, I. (1996). The looping effects of human kinds. In D. Sperber, D. Premack, \& A. J. Premack (Eds.), Causal Cognition: A Multidisciplinary Debate. Oxford University Press. https://doi.org/10.1093/acprof:oso/9780198524021.001.0001

Hacking, I. (2006). Making up people. London Review of Books, 29(16). https://www.lrb.co.uk/the-paper/v28/n16/ian-hacking/making-up-people

Hadwin, J. A., \& Kovshoff, H. (2013). A review of theory of mind interventions for children and adolescents with autism spectrum conditions. In Simon Baron-Cohen, M. Lombardo, \& H. Tager-Flusberg (Eds.), Understanding Other Minds (pp. 413-427). Oxford University Press. https://doi.org/10.1093/acprof:oso/9780199692972.003.0023

Hallmayer, J. (2011). Genetic Heritability and Shared Environmental Factors Among Twin Pairs With Autism. Archives of General Psychiatry, 68(11), 1095-1102. https://doi.org/10.1001/archgenpsychiatry.2011.76

Hannant, P., Tavassoli, T., \& Cassidy, S. (2016). The role of sensorimotor difficulties in autism spectrum conditions. Frontiers in Neurology, 7. https://doi.org/10.3389/fneur.2016.00124

Happé, F., \& Frith, U. (2006). The weak coherence account: Detail-focused cognitive style in autism spectrum disorders. Journal of Autism and Developmental Disorders, 36(1), 525. https://doi.org/10.1007/s10803-005-0039-0

Happé, F. G. E. (1994). An advanced test of theory of mind: Understanding of story characters' thoughts and feelings by able autistic, mentally handicapped, and normal children and adults. Journal of Autism and Developmental Disorders, 24(2), 129-154. https://doi.org/10.1007/BF02172093 
Happé, F. G. E. (1996). Studying weak central coherence at low levels: Children with autism do not succumb to visual illusions. Journal of Child Psychology and Psychiatry, 37(7), 873-877. https://doi.org/10.1111/j.1469-7610.1996.tb01483.x

Heasman, B., \& Gillespie, A. (2018). Perspective-taking is two-sided: Misunderstandings between people with Asperger's syndrome and their family members. Autism, 22(6), 740-750. https://doi.org/10.1177/1362361317708287

Heasman, B., \& Gillespie, A. (2019). Neurodivergent intersubjectivity: Distinctive features of how autistic people create shared understanding. Autism, 23(4), 910-921. https://doi.org/10.1177/1362361318785172

Heath, C., Hindmarsh, J., \& Luff, P. (2010). Video in qualitative research: Analysing social interaction in everyday life. SAGE Publications, Inc. https://doi.org/10.4135/9781526435385

Heath, C., \& Luff, P. (1993). Explicating face-to-face interaction. In N. Gilbert (Ed.), Researching Social Life (pp. 306-326). SAGE Publications.

Hepburn, A. (2004). Crying: Notes on description, transcription, and interaction. Research on Language \& Social Interaction, 37(3), 251-290. https://doi.org/10.1207/s15327973rlsi3703_1

Hepburn, A., \& Bolden, G. B. (2017). Transcribing for social research. SAGE Publications. Heritage, J. (2011). Conversation analysis: Practices and methods. In D. Silverman (Ed.), Qualitative research: Theory, method, and practice (3rd ed.). Sage.

Heritage, J. (1984). Garfinkel and ethnomethodology. Polity Press.

Heritage, J. (1988). Explanations as accounts: A conversation analytic perspective. In C. Antaki (Ed.), Analysing Everyday Explanation: A casebook of methods (pp. 127-144). Sage.

Heritage, J. (2012). Epistemics in action: Action formation and territories of knowledge. Research on Language \& Social Interaction, 45(1), 1-29. https://doi.org/10.1080/08351813.2012.646684

Heritage, J., Robinson, J. D., Elliott, M. N., Beckett, M., \& Wilkes, M. (2007). Reducing patients' unmet concerns in primary care: The difference one word can make. Journal of General Internal Medicine, 22(10), 1429-1433. https://doi.org/10.1007/s11606-007$\underline{0279-0}$

Hertenstein, M. J., Keltner, D., App, B., Bulleit, B. A., \& Jaskolka, A. R. (2006). Touch communicates distinct emotions. Emotion, 6(3), 528-533. https://doi.org/10.1037/1528$\underline{3542.6 .3 .528}$ 
Heslin, R., Nguyen, T. D., \& Nguyen, M. L. (1983). Meaning of touch: The case of touch from a stranger or same sex person. Journal of Nonverbal Behavior, 7(3), 147-157. https://doi.org/10.1007/BF00986945

Hill, E. L. (2004). Executive dysfunction in autism. Trends in Cognitive Sciences, 8(1), 2632. https://doi.org/10.1016/j.tics.2003.11.003

Hillary, A. (2020). Neurodiversity and cross-cultural communication. In H. Bertilsdotter Rosqvist, N. Chown, \& A. Stenning (Eds.), Neurodiversity studies: A new critical paradigm (pp. 91-107). Taylor \& Francis Group. https://www.taylorfrancis.com/books/9780429322297

Hilton, C. L., Harper, J. D., Kueker, R. H., Lang, A. R., Abbacchi, A. M., Todorov, A., \& LaVesser, P. D. (2010). Sensory responsiveness as a predictor of social severity in children with high functioning autism spectrum disorders. Journal of Autism and Developmental Disorders, 40(8), 937-945. https://doi.org/10.1007/s10803-010-0944-8

Hodges, H., Fealko, C., \& Soares, N. (2020). Autism spectrum disorder: Definition, epidemiology, causes, and clinical evaluation. Translational Pediatrics, 9(S1), S55S65. https://doi.org/10.21037/tp.2019.09.09

Hoey, E., \& Kendrick, K. (2017). Conversation analysis. In A. M. B. de Groot \& P. Hagoort (Eds.), Research methods in psycholinguistics and the neurobiology of language: A practical guide (pp. 151-173). John Wiley \& Sons Inc.

Hollin, G. (2014). Constructing a social subject: Autism and human sociality in the 1980s. History of the Human Sciences, 27(4), 98-115. https://doi.org/10.1177/0952695114528189

Hollin, G. J. S., \& Pilnick, A. (2015). Infancy, autism, and the emergence of a socially disordered body. Social Science \& Medicine, 143, 279-286. https://doi.org/10.1016/j.socscimed.2014.07.050

Hosozawa, M., Sacker, A., Mandy, W., Midouhas, E., Flouri, E., \& Cable, N. (2020). Determinants of an autism spectrum disorder diagnosis in childhood and adolescence: Evidence from the UK Millennium Cohort Study. Autism, 24(6), 1557-1565. https://doi.org/10.1177/1362361320913671

Houtkoop-Steenstra, H. (1991). Opening sequences in Dutch telephone conversations. In D. Boden \& D. Zimmerman (Eds.), Talk and Social Structure: Studies in Ethnomethodology and Conversation Analysis (pp. 232-250). Polity Press. 
Huma, B., Alexander, M., Stokoe, E., \& Tileaga, C. (2020). Introduction to special issue on discursive psychology. Qualitative Research in Psychology, 17(3), 313-335. https://doi.org/10.1080/14780887.2020.1729910

Hurtig, R., Ensrud, S., \& Tomblin, J. B. (1982). The communicative function of question production in autistic children. Journal of Autism and Developmental Disorders, 12(1), 57-69. https://doi.org/10.1007/BF01531674

Hutchby, I. (2002). Resisting the incitement to talk in child counselling: Aspects of the utterance 'I don't know'. Discourse Studies, 4(2), 147-168. https://doi.org/10.1177/14614456020040020201

Hutchby, I., \& Wooffitt, R. (2008). Conversation analysis (2nd ed). Polity. James, A., Jenks, C., \& Prout, A. (1998). Theorizing childhood (Repr). Polity Press.

Jarrold, C., Boucher, J., \& Smith, P. (1993). Symbolic play in autism: A review. Journal of Autism and Developmental Disorders, 23(2), 281-307. https://doi.org/10.1007/BF01046221

Jefferson, G. (1993). Caveat speaker: Preliminary notes on recipient topic-shift implicature. Research on Language \& Social Interaction, 26(1), 1-30. https://doi.org/10.1207/s15327973rlsi2601_1

Jenkins, L., \& Hepburn, A. (2015). Children's sensations as interactional phenomena: A conversation analysis of children's xxpressions of pain and discomfort. Qualitative Research in Psychology, 12(4), 472-491. https://doi.org/10.1080/14780887.2015.1054534

Johnson, N. L., Bekhet, A., Robinson, K., \& Rodriguez, D. (2014). Attributed meanings and strategies to prevent challenging behaviors of hospitalized children with autism: Two perspectives. Journal of Pediatric Health Care, 28(5), 386-393. https://doi.org/10.1016/j.pedhc.2013.10.001

Jones, R. S. P., Quigney, C., \& Huws, J. C. (2003). First-hand accounts of sensory perceptual experiences in autism: A qualitative analysis. Journal of Intellectual \& Developmental Disability, 28(2), 112-121. https://doi.org/10.1080/1366825031000147058

Juranek, J., Filipek, P. A., Berenji, G. R., Modahl, C., Osann, K., \& Spence, M. A. (2006). Association Between Amygdala Volume and Anxiety Level: Magnetic Resonance Imaging (MRI) Study in Autistic Children. Journal of Child Neurology, 21(12), 10511058. https://doi.org/10.1177/7010.2006.00237

Kaiser, M. D., Yang, D. Y.-J., Voos, A. C., Bennett, R. H., Gordon, I., Pretzsch, C., Beam, D., Keifer, C., Eilbott, J., McGlone, F., \& Pelphrey, K. A. (2016). Brain mechanisms 
for processing affective (and nonaffective) touch are atypical in autism. Cerebral Cortex, 26(6), 2705-2714. https://doi.org/10.1093/cercor/bhv125

Kanner, L. (1943). Autistic disturbances of affective contact. Nervous Child, 2, 217-250.

Karim, K. (2017). Autism spectrum disorder: An introduction. In M. O’Reilly, J. N. Lester, \& T. Muskett (Eds.), A Practical Guide to Social Interaction Research in Autism Spectrum Disorders (pp. 33-60). Palgrave Macmillan UK. https://doi.org/10.1057/978$\underline{1-137-59236-1 \_2}$

Keel, S. (2016). Socialization: Parent-child interaction in everyday life.

Kelley, E., Paul, J. J., Fein, D., \& Naigles, L. R. (2006). Residual language deficits in optimal outcome children with a history of autism. Journal of Autism and Developmental Disorders, 36(6), 807-828. https://doi.org/10.1007/s10803-006-0111-4

Kendon, A. (1990). Conducting interaction: Patterns of behavior in focused encounters. Cambridge University Press.

Kendrick, K. H. (2017). Using conversation analysis in the lab. Research on Language and Social Interaction, 50(1), 1-11. https://doi.org/10.1080/08351813.2017.1267911

Kenny, L., Hattersley, C., Molins, B., Buckley, C., Povey, C., \& Pellicano, E. (2016). Which terms should be used to describe autism? Perspectives from the UK autism community. Autism, 20(4), 442-462. https://doi.org/10.1177/1362361315588200

Kent, A. (2012). Compliance, resistance and incipient compliance when responding to directives. Discourse Studies, 14(6), 711-730. https://doi.org/10.1177/1461445612457485

Kent, Alexandra. (2011). Directing dinnertime: Practices and resources used by parents and children to deliver and respond to directive actions [Loughborough University]. https://dspace.lboro.ac.uk/dspacejspui/bitstream/2134/8960/1/Kent\%20PhD\%20Thesis\%20Final.pdf

Kent, Alexandra. (2012). Responding to directives: What can children do when a parent tells them what to do? In S. Danby \& M. Theobald (Eds.), Sociological Studies of Children and Youth (Vol. 15, pp. 57-84). Emerald Group Publishing Limited. https://doi.org/10.1108/S1537-4661(2012)0000015007

Kern, J. K., Trivedi, M. H., Garver, C. R., Grannemann, B. D., Andrews, A. A., Savla, J. S., Johnson, D. G., Mehta, J. A., \& Schroeder, J. L. (2006). The pattern of sensory processing abnormalities in autism. Autism, 10(5), 480-494. https://doi.org/10.1177/1362361306066564 
Kidwell, M. (2005). Gaze as social control: How very young children differentiate "the look" from a "mere look" by their adult caregivers. Research on Language \& Social Interaction, 38(4), 417-449. https://doi.org/10.1207/s15327973rlsi3804_2

Kim, S. H., Paul, R., Tager-Flusberg, H., \& Lord, C. (2014). Language and communication in Autism. In Handbook of autism and pervasive developmental disorders: Diagnosis, development and brain mechanisms (4th ed., Vol. 1, pp. 230-262). John Wiley and Sons.

Kimhi, Y. (2014). Theory of mind abilities and deficits in autism spectrum disorders. Topics in Language Disorders, 34(4), 329-343. https://doi.org/10.1097/TLD.0000000000000033

Kissine, M., Cano-Chervel, J., Carlier, S., De Brabanter, P., Ducenne, L., Pairon, M.-C., Deconinck, N., Delvenne, V., \& Leybaert, J. (2015). Children with autism understand indirect speech acts: Evidence from a semi-structured act-out task. PLOS ONE, 1O(11), e0142191. https://doi.org/10.1371/journal.pone.0142191

Kissine, M., De Brabanter, P., \& Leybaert, J. (2012). Compliance with requests by children with autism: The impact of sentence type. Autism, 16(5), 523-531. https://doi.org/10.1177/1362361311406296

Kjelgaard, M. M., \& Tager-Flusberg, H. (2001). An investigation of language impairment in autism: Implications for genetic subgroups. Language and Cognitive Processes, 16(23), 287-308. https://doi.org/10.1080/01690960042000058

Korkiakangas, T., Dindar, K., Laitila, A., \& Kärnä, E. (2016). The Sally-Anne test: An interactional analysis of a dyadic assessment: Sally-Anne test: an interactional analysis. International Journal of Language \& Communication Disorders, 51(6), 685-702. https://doi.org/10.1111/1460-6984.12240

Korkiakangas, T. K., Rae, J. P., \& Dickerson, P. (2012). The interactional work of repeated talk between a teacher and a child with autism. Journal of Interactional Research in Communication Disorders, 3(1), 1-25. https://doi.org/10.1558/jircd.v3i1.1

Korkiakangas, T., \& Rae, J. (2014). The interactional use of eye-gaze in children with autism spectrum disorders. Interaction Studies, 15(2), 233-259. https://doi.org/10.1075/is.15.2.12kor

Kremer-Sadlik, T. (2004). How Children with Autism and Asperger Syndrome Respond to Questions: A 'Naturalistic' Theory of Mind Task. Discourse Studies, 6(2), 185-206. https://doi.org/10.1177/1461445604041767 
Kupetz, M. (2019). Embodying Empathy in Everyday and Institutional Settings: On the Negotiation of Resources, Rights, and Responsibilities in Comforting Actions. In E. Reber \& C. Gerhardt (Eds.), Embodied Activities in Face-to-face and Mediated Settings (pp. 329-367). Springer International Publishing. https://doi.org/10.1007/978-3-319$\underline{97325-8 \_10}$

Kyratzis, A., \& Goodwin, M. H. (2017). Language Socialization in Children's Peer and Sibling-Kin Group Interactions. In P. A. Duff \& S. May (Eds.), Language Socialization (pp. 1-16). Springer International Publishing. https://doi.org/10.1007/978-3-319$\underline{02327-4 \_10-1}$

Lamerichs, J., Alisic, E., \& Schasfoort, M. (2018). Accounts and their epistemic implications. Research on Children and Social Interaction, 2(1), 25-48. https://doi.org/10.1558/rcsi.35244

Larson, E. (2006). Caregiving and Autism: How Does Children's Propensity for Routinization Influence Participation in Family Activities? OTJR: Occupation, Participation and Health, 26(2), 69-79. https://doi.org/10.1177/153944920602600205 Laurier, E., \& Philo, C. (2006). Natural problems of naturalistic video data. In H. Knoblauch, J. Raab, H.-G. Soeffner, \& B. Schnettler (Eds.), Video Analysis (pp. 183-192). Peter Lang.

Le Couteur, A., Lord, C., \& Rutter, M. (2003). Autism diagnostic interview-revised. Western Psychological Services.

Leary, M. R., \& Hill, D. A. (1996). Moving on: Autism and movement disturbance. Mental Retardation, 34(1), 39-53.

Lerner, G. H. (2003). Selecting next speaker: The context-sensitive operation of a contextfree organization. Language in Society, 32(02), 177-201. https://doi.org/10.1017/S004740450332202X

Leslie, A. M. (1987). Pretense and representation: The origins of "theory of mind." Psychological Review, 94(4), 412-426. https://doi.org/10.1037/0033-295X.94.4.412

Leung, R. C., Vogan, V. M., Powell, T. L., Anagnostou, E., \& Taylor, M. J. (2016). The role of executive functions in social impairment in Autism Spectrum Disorder. Child Neuropsychology, 22(3), 336-344. https://doi.org/10.1080/09297049.2015.1005066

Lindström, A. (1994). Identification and recognition in Swedish telephone conversation openings. Language in Society, 23(2), 231-252. https://doi.org/10.1017/S004740450001784X 
Livingston, L. A., Colvert, E., Social Relationships Study Team, Bolton, P., \& Happé, F. (2019). Good social skills despite poor theory of mind: Exploring compensation in autism spectrum disorder. Journal of Child Psychology and Psychiatry, and Allied Disciplines, 60(1), 102-110. https://doi.org/10.1111/jcpp.12886

Livingston, L. A., \& Happé, F. (2017). Conceptualising compensation in neurodevelopmental disorders: Reflections from autism spectrum disorder. Neuroscience and Biobehavioral Reviews, 80, 729-742. https://doi.org/10.1016/j.neubiorev.2017.06.005

Livingston, L. A., Shah, P., \& Happé, F. (2019). Compensatory strategies below the behavioural surface in autism: A qualitative study. The Lancet Psychiatry, 6(9), 766777. https://doi.org/10.1016/S2215-0366(19)30224-X

Livingston, L. A., Shah, P., Milner, V., \& Happé, F. (2020). Quantifying compensatory strategies in adults with and without diagnosed autism. Molecular Autism, 11(1), 15. https://doi.org/10.1186/s13229-019-0308-y

Local, J., \& Wootton, T. (1995). Interactional and phonetic aspects of immediate echolalia in autism: A case study. Clinical Linguistics \& Phonetics, 9(2), 155-184. https://doi.org/10.3109/02699209508985330

Lomax, H., \& Casey, N. (1998). Recording Social Life: Reflexivity and Video Methodology. Sociological Research Online, 3(2), 121-146. https://doi.org/10.5153/sro.1372

Lombardo, M. V., \& Baron-Cohen, S. (2010). Unraveling the paradox of the autistic self. Wiley Interdisciplinary Reviews: Cognitive Science, 1(3), 393-403. https://doi.org/10.1002/wcs.45

Lord, C., Rutter, M., DiLavore, P., Risi, S., Gotham, K., \& Bishop, S. (2012). Autism diagnostic observation schedule, (ADOS-2) modules 1-4. Los Angeles, California: Western Psychological Services.

Lorde, A. (1984). Sister outsider: Essays and speeches. Crossing Press.

Loveland, K. A., \& Landry, S. H. (1986). Joint attention and language in autism and developmental language delay. Journal of Autism and Developmental Disorders, 16(3), $335-349$.

Loveland, Katherine A., Landry, S. H., Hughes, S. O., Hall, S. K., \& McEvoy, R. E. (1988). Speech Acts and the Pragmatic Deficits of Autism. Journal of Speech Language and Hearing Research, 31(4), 593. https://doi.org/10.1044/jshr.3104.593

Lundqvist, L.-O. (2015). Hyper-responsiveness to touch mediates social dysfunction in adults with autism spectrum disorders. Research in Autism Spectrum Disorders, 9, 13-20. https://doi.org/10.1016/j.rasd.2014.09.012 
Lynch, M. (1984). “Turning up Signs” in Neurobehavioral Diagnosis*. Symbolic Interaction, 7(1), 67-86. https://doi.org/10.1525/si.1984.7.1.67

Lynch, M. (1985). Discipline and the Material Form of Images: An Analysis of Scientific Visibility. Social Studies of Science, 15(1), 37-66. https://doi.org/10.1177/030631285015001002

Lytton, H. (1971). Observation studies of parent-child interaction: A methodological review. Child Development, 42(3), 651-684. https://doi.org/10.2307/1127439

Mackay, R. (1991). Conceptions of children and models of socialization. In F. C. Waksler (Ed.), Studying The Social Worlds Of Children: Sociological Readings (pp. 23-37). Palmer Press.

Maclaren, K. (2014). Touching matters: Embodiments of intimacy. Emotion, Space and Society, 13, 95-102. https://doi.org/10.1016/j.emospa.2013.12.004

Maenner, M. J., Shaw, K. A., Baio, J., EdS1, Washington, A., Patrick, M., DiRienzo, M., Christensen, D. L., Wiggins, L. D., Pettygrove, S., Andrews, J. G., Lopez, M., Hudson, A., Baroud, T., Schwenk, Y., White, T., Rosenberg, C. R., Lee, L.-C., Harrington, R. A., ... Dietz, P. M. (2020). Prevalence of autism spectrum disorder among children aged 8 years. MMWR. Surveillance Summaries, 69(4), 1-12. https://doi.org/10.15585/mmwr.ss6904a1

Mammen, M. A., Moore, G. A., Scaramella, L. V., Reiss, D., Ganiban, J. M., Shaw, D. S., Leve, L. D., \& Neiderhiser, J. M. (2015). Infant avoidance during a tactile task predicts autism spectrum disorders in childhood: Infant avoidance during a tactile task. Infant Mental Health Journal, 36(6), 575-587. https://doi.org/10.1002/imhj.21539

Mannion, A., \& Leader, G. (2013). An analysis of the predictors of comorbid psychopathology, gastrointestinal symptoms and epilepsy in children and adolescents with autism spectrum disorder. Research in Autism Spectrum Disorders, 7(12), 16631671. https://doi.org/10.1016/j.rasd.2013.10.002

Maracek, J. (2003). Dancing through minefields: Toward a qualitative stance in psychology. In P. Camic, J. Rhodes, \& L. Yardley (Eds.), Qualitative Research In Psychology: Expanding perspectives in Methodology and design (pp. 49-69). Americvan Psychological Association.

Margoni, F., \& Surian, L. (2016). Mental state understanding and moral judgment in children with autistic spectrum disorder. Frontiers in Psychology, 7, 1478. https://doi.org/10.3389/fpsyg.2016.01478 
Marstrand, A. K., \& Svennevig, J. (2018). A preference for non-invasive touch in caregiving contexts. Social Interaction. Video-Based Studies of Human Sociality, 1(2). https://doi.org/10.7146/si.v1i2.110019

Masi, A., DeMayo, M. M., Glozier, N., \& Guastella, A. J. (2017). An overview of autism spectrum disorder, heterogeneity and treatment options. Neuroscience Bulletin, 33(2), 183-193. https://doi.org/10.1007/s12264-017-0100-y

Maynard, D. W. (1980). Placement of topic changes in conversation. Semiotica, 30(3-4), 263-290. https://doi.org/10.1515/semi.1980.30.3-4.263

Maynard, D. W., \& Turowetz, J. (2017a). Doing diagnosis: Autism, interaction order, and the use of narrative in clinical talk. Social Psychology Quarterly, 80(3), 254-275. https://doi.org/10.1177/0190272517720683

Maynard, D. W., \& Turowetz, J. J. (2017b). Doing testing: How concrete competence can facilitate or inhibit performances of children with autism spectrum disorder. Qualitative Sociology, 40(4), 467-491. https://doi.org/10.1007/s11133-017-9368-5

McCabe, R., Leudar, I., \& Antaki, C. (2004). Do people with schizophrenia display theory of mind deficits in clinical interactions? Psychological Medicine, 34(3), 401-412. https://doi.org/10.1017/S0033291703001338

McCann, J., \& Peppé, S. (2003). Prosody in autism spectrum disorders: A critical review. International Journal of Language \& Communication Disorders, 38(4), 325-350. https://doi.org/10.1080/1368282031000154204

Meirsschaut, M., Roeyers, H., \& Warreyn, P. (2010). Parenting in families with a child with autism spectrum disorder and a typically developing child: Mothers' experiences and cognitions. Research in Autism Spectrum Disorders, 4(4), 661-669. https://doi.org/10.1016/j.rasd.2010.01.002

Milton, D. (2013). 'Filling in the Gaps': A micro-sociological analysis of autism. Autonomy, the Critical Journal of Interdisciplinary Autism Studies, 1(2). http://www.larryarnold.net/Autonomy/index.php/autonomy/article/view/7

Milton, D. (2014). Embodied sociality and the conditioned relativism of dispositional diversity. Autonomy, the Critical Journal of Interdisciplinary Autism Studies, 1(3). http://www.larry-arnold.net/Autonomy/index.php/autonomy/article/view/AR10/html Milton, D., \& Bracher, M. (2013). Autistics speak out but are the heard? Medical Sociology Online, 7(2), 63-69. 
Milton, Damian Elgin Maclean, Heasman, B., \& Sheppard, E. (2018). Double empathy. In F. R. Volkmar (Ed.), Encyclopedia of Autism Spectrum Disorders (pp. 1-8). Springer New York. https://doi.org/10.1007/978-1-4614-6435-8_102273-1

Milton, Damian E.M. (2012). On the ontological status of autism: The 'double empathy problem.' Disability \& Society, 27(6), 883-887. https://doi.org/10.1080/09687599.2012.710008

Moncrieff, J. (2010). Psychiatric diagnosis as a political device. Social Theory \& Health, 8(4), 370-382. https://doi.org/10.1057/sth.2009.11

Mondada, L. (2006). Video recording as the reflexive preservation and configuration of phenomenal features for analysis. In H. Knoblauch, J. Raab, H.-G. Soeffner, \& B. Schnettler (Eds.), Video Analysis (pp. 51-68). Lang.

Mondada, L. (2016a). Conventions for multimodal transcription. https://franzoesistik.philhist.unibas.ch/fileadmin/user_upload/franzoesistik/mondada_m ultimodal_conventions.pdf

Mondada, L. (2016b). Challenges of multimodality: Language and the body in social interaction. Journal of Sociolinguistics, 20(3), 336-366. https://doi.org/10.1111/josl.1_12177

Mondada, L. (2019). Rethinking bodies and objects in social interaction: A multimodal and multisensorial approach to tasting. In U. T. Kissmann \& J. van Loon (Eds.), Discussing New Materialism (pp. 109-134). Springer Fachmedien Wiesbaden. https://doi.org/10.1007/978-3-658-22300-7_6

Moore, M. L., Eichner, S. F., \& Jones, J. R. (2004). Treating Functional Impairment of Autism with Selective Serotonin-Reuptake Inhibitors. Annals of Pharmacotherapy, 38(9), 1515-1519. https://doi.org/10.1345/aph.1D543

Morrison, K. E., DeBrabander, K. M., Faso, D. J., \& Sasson, N. J. (2019). Variability in first impressions of autistic adults made by neurotypical raters is driven more by characteristics of the rater than by characteristics of autistic adults: Autism, 1817-1829. https://doi.org/10.1177/1362361318824104

Moss, P., Mandy, W., \& Howlin, P. (2017). Child and adult factors related to quality of life in adults with autism. Journal of Autism and Developmental Disorders, 47(6), 1830-1837. https://doi.org/10.1007/s10803-017-3105-5

Muskett, T., Perkins, M., Clegg, J., \& Body, R. (2010). Inflexibility as an interactional phenomenon: Using conversation analysis to re-examine a symptom of autism. Clinical Linguistics \& Phonetics, 24(1), 1-16. https://doi.org/10.3109/02699200903281739 
Nadesan, M. H. (2013). Constructing Autism: Unravelling the "truth" and understanding the social. Routledge. https://doi.org/10.4324/9780203299500

Neely-Barnes, S. L., Hall, H. R., Roberts, R. J., \& Graff, J. C. (2011). Parenting a child with an autism spectrum disorder: Public perceptions and parental conceptualizations.

Journal of Family Social Work, 14(3), 208-225.

https://doi.org/10.1080/10522158.2011.571539

Neurodivergent K. (2011, April 11). What would meeting you halfway be? Radical Neurodivergence Speaking. http://timetolisten.blogspot.com/2011/04/what-wouldmeeting-you-halfway-be.html

Nevile, M. (2015). The embodied turn in research on language and social interaction.

Research on Language and Social Interaction, 48(2), 121-151.

https://doi.org/10.1080/08351813.2015.1025499

New Zealand Ministries of Health and Education. (2016). New Zealand autism spectrum disorder: Guideline. (2nd ed.).

Nguyen, M., Roth, A., Kyzar, E. J., Poudel, M. K., Wong, K., Stewart, A. M., \& Kalueff, A. V. (2014). Decoding the contribution of dopaminergic genes and pathways to autism spectrum disorder (ASD). Neurochemistry International, 66, 15-26. https://doi.org/10.1016/j.neuint.2014.01.002

Nishizaka, A. (2007). Hand touching hand: Referential practice at a japanese midwife house. Human Studies, 30(3), 199-217. https://doi.org/10.1007/s10746-007-9059-4

Nishizaka, A. (2011). Touch without vision: Referential practice in a non-technological environment. Journal of Pragmatics, 43(2), 504-520. https://doi.org/10.1016/j.pragma.2009.07.015

Ochs, E. (2002). Becoming a speaker of a culture. In C. Kramsch (Ed.), Language Acquisition and Language Socialization (pp. 99-120). Continuum.

Ochs, E. (2015). Corporeal reflexivity and autism. Integrative Psychological and Behavioral Science, 49(2), 275-287. https://doi.org/10.1007/s12124-015-9306-6

Ochs, E., Kremer-Sadlik, T., Sirota, K. G., \& Solomon, O. (2004b). Autism and the social world: An anthropological perspective. Discourse Studies, 6(2), 147-183. https://doi.org/10.1177/1461445604041766

Ochs, E., \& Solomon, O. (2005). Practical logic and autism. In C. Casey \& R. B. Edgerton (Eds.), A Companion to Psychological Anthropology (pp. 140-167). Blackwell Publishing Ltd. https://doi.org/10.1002/9780470996409.ch9 
Ochs, E., \& Solomon, O. (2010). Autistic sociality. Ethos, 38(1), 69-92. https://doi.org/10.1111/j.1548-1352.2009.01082.x

Oliver, M. (1990). The politics of disablement. Macmillan.

Oliver, M. (1997). Emancipatory research: Realistic goal or impossible dream? In C. Barnes \& G. Mercer (Eds.), Doing disability research (pp. 15-31). Disability Press.

O’Nions, E., Happé, F., Evers, K., Boonen, H., \& Noens, I. (2018). How do parents manage irritability, challenging behaviour, non-compliance and anxiety in children with autism spectrum disorders? A Meta-Synthesis. Journal of Autism and Developmental Disorders, 48(4), 1272-1286. https://doi.org/10.1007/s10803-017-3361-4

Ooi, K., Ong, Y. S., Jacob, S. A., \& Khan, T. M. (2016). A meta-synthesis on parenting a child with autism. Neuropsychiatric Disease and Treatment, 745. https://doi.org/10.2147/NDT.S100634

O’Reilly, M., Kiyimba, N., Nina Lester, J., \& Muskett, T. (2020). Reflective interventionist conversation analysis. Discourse \& Communication, 14(6), 619-634. https://doi.org/10.1177/1750481320939710

O’Reilly, C., Lewis, J. D., \& Elsabbagh, M. (2017). Is functional brain connectivity atypical in autism? A systematic review of EEG and MEG studies. PLOS ONE, 12(5), e0175870. https://doi.org/10.1371/journal.pone.0175870

Orsini, M., \& Davidson, J. (2013). Introduction. In M. Orsini \& J. Davidson (Eds.), Worlds of Autism (pp. 1-28). University of Minnesota Press; JSTOR. http://www.jstor.org/stable/10.5749/j.ctt4cggsn.4

Orsmond, G. I., Shattuck, P. T., Cooper, B. P., Sterzing, P. R., \& Anderson, K. A. (2013). Social participation among young adults with an autism spectrum disorder. Journal of Autism and Developmental Disorders, 43(11), 2710-2719. https://doi.org/10.1007/s10803-013-1833-8

Ozonoff, S., \& Jensen, J. (1999). Specific executive function profiles in three neurodevelopmental disorders. Journal of Autism and Developmental Disorders, 29(2), 171-177. https://doi.org/10.1023/A:1023052913110

Paul, R., Augustyn, A., Klin, A., \& Volkmar, F. R. (2005). Perception and production of prosody by speakers with autism spectrum disorders. Journal of Autism and Developmental Disorders, 35(2), 205-220. https://doi.org/10.1007/s10803-004-1999-1

Peled-Avron, L., \& Shamay-Tsoory, S. G. (2017). Don't touch me! autistic traits modulate early and late ERP components during visual perception of social touch: Autistic traits 
modulate ERP for social touch. Autism Research, 10(6), 1141-1154. https://doi.org/10.1002/aur.1762

Pellicano, E. (2010). Individual differences in executive function and central coherence predict developmental changes in theory of mind in autism. Developmental Psychology, 46(2), 530-544. https://doi.org/10.1037/a0018287

Pellicano, E., Dinsmore, A., \& Charman, T. (2014). What should autism research focus upon? Community views and priorities from the United Kingdom. Autism, 18(7), 756770. https://doi.org/10.1177/1362361314529627

Pellicano, L., Mandy, W., Bölte, S., Stahmer, A., Lounds Taylor, J., \& Mandell, D. S. (2018). A new era for autism research, and for our journal. Autism, 22(2), 82-83. https://doi.org/10.1177/1362361317748556

Pennington, B. F., \& Ozonoff, S. (1996). Executive functions and developmental psychopathology. Journal of Child Psychology and Psychiatry, 37(1), 51-87. https://doi.org/10.1111/j.1469-7610.1996.tb01380.x

Pickles, A., Starr, E., Kazak, S., Bolton, P., Papanikolaou, K., Bailey, A., Goodman, R., \& Rutter, M. (2000). Variable expression of the autism broader phenotype: Findings from extended pedigrees. Journal of Child Psychology and Psychiatry, and Allied Disciplines, 41(4), 491-502.

Pierce, K., Gazestani, V. H., Bacon, E., Barnes, C. C., Cha, D., Nalabolu, S., Lopez, L., Moore, A., Pence-Stophaeros, S., \& Courchesne, E. (2019). Evaluation of the Diagnostic Stability of the Early Autism Spectrum Disorder Phenotype in the General Population Starting at 12 Months. JAMA Pediatrics, 173(6), 578. https://doi.org/10.1001/jamapediatrics.2019.0624

Pontecorvo, C., Fasulo, A., \& Sterponi, L. (2001). Mutual apprentices: The making of parenthood and childhood in family dinner conversations. Human Development, 44(6), 340-361. https://doi.org/10.1159/000046155

Potter, J. (2002). Two kinds of natural. Discourse Studies, 4(4), 539-542. https://doi.org/10.1177/14614456020040040901

Potter, J., Edwards, D., \& Wetherell, M. (1993). A model of discourse in action. American Behavioral Scientist, 36(3), 383-401. https://doi.org/10.1177/0002764293036003008

Potter, J., \& Shaw, C. (2018). The virtues of naturalistic data. In U. Flick (Ed.), The Sage handbook of qualitative data collection (pp. 182-199). Sage. 
Premack, D., \& Woodruff, G. (1978). Does the chimpanzee have a theory of mind?

Behavioral and Brain Sciences, 1(4), 515-526. https://doi.org/10.1017/S0140525X00076512

Raymond, G. (2004). Prompting action: She stand-alone "so" in ordinary conversation. Research on Language \& Social Interaction, 37(2), 185-218. https://doi.org/10.1207/s15327973rlsi3702_4

Rendle-Short, J. (2002). Managing interaction: A conversation analytic approach to the management of interaction by an 8 year-old girl with Asperger's Syndrome. Issues in Applied Linguistics, 13(2), 161-186.

Rendle-Short, J. (2007). "Catherine, you're wasting your time”: Address terms within the Australian political interview. Journal of Pragmatics, 39(9), 1503-1525. https://doi.org/10.1016/j.pragma.2007.02.006

Rendle-Short, J. (2014). Chapter 9. Using conversational structure as an interactional resource: Children with Asperger's syndrome and their conversational partners. In J. Arciuli \& J. Brock (Eds.), Trends in Language Acquisition Research (Vol. 11, pp. $217-$ 244). John Benjamins Publishing Company. https://doi.org/10.1075/tilar.11.10ren

Rendle-Short, J. (2019). Analysing atypical interaction: Reflections on the intersection between quantitative and qualitative research. Journal of Pragmatics, 143, 267-278. https://doi.org/10.1016/j.pragma.2018.04.009

Rendle-Short, J., Wilkinson, R., \& Danby, S. (2015). Name-calling by a child with Asperger's syndrome. In M. O'Reilly \& J. N. Lester (Eds.), The Palgrave Handbook of Child Mental Health: Discourse and Conversation Studies (pp. 350-366). Palgrave Macmillan UK. https://doi.org/10.1057/9781137428318_19

Reynolds, E., \& Fitzgerald, R. (2015). Challenging normativity re-appraising category bound, tied and predicated features. In R. Fitzgerald \& W. Housley, Advances in Membership Categorisation Analysis (pp. 99-122). SAGE Publications Ltd. https://doi.org/10.4135/9781473917873.n5

Riquelme, I., Hatem, S. M., \& Montoya, P. (2016). Abnormal pressure pain, touch sensitivity, proprioception, and manual dexterity in children with autism spectrum disorders. Neural Plasticity, 2016, 1-9. https://doi.org/10.1155/2016/1723401

Rose, N. (1988). Calculable minds and manageable individuals. History of the Human Sciences, 1(2), 179-200. https://doi.org/10.1177/095269518800100202

Rosenberg, R. E., Law, J. K., Yenokyan, G., McGready, J., Kaufmann, W. E., \& Law, P. A. (2009). Characteristics and Concordance of Autism Spectrum Disorders Among 277 
Twin Pairs. Archives of Pediatrics \& Adolescent Medicine, 163(10), 907. https://doi.org/10.1001/archpediatrics.2009.98

Rozin, P. (2001). Social psychology and science: Some lessons from Solomon Asch.

Personality and Social Psychology Review, 5(1), 2-14. https://doi.org/10.1207/S15327957PSPR0501_1

Russell-Smith, S. N., Maybery, M. T., Bayliss, D. M., \& Sng, A. A. H. (2012). Support for a link between the local processing bias and social deficits in autism: an investigation of embedded figures test performance in non-clinical individuals. Journal of Autism and Developmental Disorders, 42(11), 2420-2430. https://doi.org/10.1007/s10803-012$\underline{1506-\mathrm{Z}}$

Rutter, M. (1978). Diagnosis and definition of childhood autism. Journal of Autism and Childhood Schizophrenia, 8(2), 139-161.

Sacks, H. (1967). The search for help: No one to turn to. In E. Schneidman (Ed.), Essays in Self-Destruction (pp. 203-223). Science House.

Sacks, H. (1974). On the analyzability of stories by children. In R. Turner (Ed.), Ethnomethodology (pp. 216-232). Penguin.

Sacks, H. (1985). On doing “being ordinary.” In J. M. Atkinson (Ed.), Structures of Social Action (pp. 413-429). Cambridge University Press. https://doi.org/10.1017/CBO9780511665868.024

Sacks, H. (1995). Lectures on conversation: Volumes I \& II. Blackwell.

Sacks, H., Schegloff, E. A., \& Jefferson, G. (1974). A simplest systematics for the organization of turn-taking for conversation. Language, 50(4), 696. https://doi.org/10.2307/412243

Sanders, R. E. (2005). Validating 'observations' in discourse studies: A methodological reason for attention to cognition. In H. te Molder \& J. Potter (Eds.), Conversation and Cognition (1st ed., pp. 57-78). Cambridge University Press. https://doi.org/10.1017/CBO9780511489990.003

Sasson, N. J., Faso, D. J., Nugent, J., Lovell, S., Kennedy, D. P., \& Grossman, R. B. (2017). Neurotypical peers are less willing to interact with those with autism based on thin slice judgments. Scientific Reports, 7(1), 40700. https://doi.org/10.1038/srep40700

Sasson, N. J., \& Morrison, K. E. (2019). First impressions of adults with autism improve with diagnostic disclosure and increased autism knowledge of peers. Autism, 23(1), 50-59. https://doi.org/10.1177/1362361317729526 
Schegloff, E. (1979). Identification and recognition in telephone conversation openings. In G. Psathas (Ed.), Everyday Language: Studies in Ethnomethodology (pp. 23-78). Irvington Publishers.

Schegloff, E. A. (1968). Sequencing in conversational openings. American Anthropologist, 70(6), 1075-1095. https://doi.org/10.1525/aa.1968.70.6.02a00030

Schegloff, E. A. (1987). Analyzing single episodes of interaction: an exercise in conversation analysis. Social Psychology Quarterly, 50(2), 101. https://doi.org/10.2307/2786745

Schegloff, E. A. (1992). Repair after next turn: The last structurally provided defense of intersubjectivity in conversation. American Journal of Sociology, 97(5), 1295-1345. https://doi.org/10.1086/229903

Schegloff, E. A. (1995). Discourse as an interactional achievement iii: The omnirelevance of action. Research on Language and Social Interaction, 28(3), 185-211. https://doi.org/10.1207/s15327973rlsi2803_2

Schegloff, E. A. (1996). Confirming allusions: Toward an empirical account of action. American Journal of Sociology, 102(1), 161-216. JSTOR.

Schegloff, E. A. (2007). Sequence organization in interaction: A primer in conversation analysis. Cambridge University Press. https://doi.org/10.1017/CBO9780511791208

Schiffrin, D. (1996). Discourse markers (1st paperback ed., repr). Cambridge Univ. Press.

Schopler, E., Reichler, R. J., \& Renner, B. R. (1986). The childhood autism rating scale (CARS): For diagnostic screening and classification of autism. Irvington.

Scott, M. B., \& Lyman, S. M. (1968). Accounts. American Sociological Review, 33(1), 4662. JSTOR. https://doi.org/10.2307/2092239

Shumway, S., \& Wetherby, A. M. (2009). Communicative acts of children with autism spectrum disorders in the second year of life. Journal of Speech Language and Hearing Research, 52(5), 1139. https://doi.org/10.1044/1092-4388(2009/07-0280)

Sidnell, J. (2013). Basic conversation analytic methods. In J. Sidnell \& T. Stivers (Eds.), The Handbook of Conversation Analysis (pp. 76-99). Wiley-Blackwell.

Silva, L. M. T., Schalock, M., \& Gabrielsen, K. R. (2015). About face: Evaluating and managing tactile impairment at the time of autism diagnosis. Autism Research and Treatment, 2015, 1-10. https://doi.org/10.1155/2015/612507

Silva, L., \& Schalock, M. (2016). First skin biopsy reports in children with autism show loss of c-tactile fibers. Journal of Neurological Disorders, 04(02). https://doi.org/10.4172/2329-6895.1000262 
Sirota, K. G. (2006). Habits of the hearth: Children's bedtime routines as relational work. Text \& Talk - An Interdisciplinary Journal of Language, Discourse Communication Studies, 26(4-5), 493-514. https://doi.org/10.1515/TEXT.2006.020

Smirni, D., Smirni, P., Carotenuto, M., Parisi, L., Quatrosi, G., \& Roccella, M. (2019). Noli me tangere: Social touch, tactile defensiveness, and communication in neurodevelopmental disorders. Brain Sciences, 9(12), 368. https://doi.org/10.3390/brainsci9120368

Sng, C. Y., Carter, M., Stephenson, J., \& Sweller, N. (2020). Partner perceptions of conversations with individuals with autism spectrum disorder. Journal of Autism and Developmental Disorders, 50(4), 1182-1197. https://doi.org/10.1007/s10803-019$\underline{04348-8}$

Soke, G. N., Maenner, M. J., Christensen, D., Kurzius-Spencer, M., \& Schieve, L. A. (2018). Prevalence of co-occurring medical and behavioral conditions/symptoms among 4- and 8 -year-old children with autism spectrum disorder in selected areas of the united states in 2010. Journal of Autism and Developmental Disorders, 48(8), 2663-2676. https://doi.org/10.1007/s10803-018-3521-1

Solomon, O. (2004). Narrative introductions: Discourse competence of children with autistic spectrum disorders. Discourse Studies, 6(2), 253-276. https://doi.org/10.1177/1461445604041770

Sparrman, A. (2005). Video recording as interaction: Participant observation of children's everyday life. Qualitative Research in Psychology, 2(3), 241-255. https://doi.org/10.1191/1478088705qp041oa

Speer, S. A. (2002). Sexist talk: Gender categories, participants' orientations and irony. Journal of Sociolinguistics, 6(3), 347-377. https://doi.org/10.1111/1467-9481.00192

Staal, W. G. (2015). Autism, DRD3 and repetitive and stereotyped behavior, an overview of the current knowledge. European Neuropsychopharmacology, 25(9), 1421-1426. https://doi.org/10.1016/j.euroneuro.2014.08.011

Sterponi, L. (2004). Construction of rules, accountability and moral identity by highfunctioning children with autism. Discourse Studies, 6(2), 207-228. https://doi.org/10.1177/1461445604041768

Sterponi, L. (2009). Accountability in family discourse: Socialization into norms and standards and negotiation of responsibility in italian dinner conversations. Childhood, 16(4), 441-459. https://doi.org/10.1177/0907568209343269 
Sterponi, L. A. (2003). Account episodes in family discourse: The making of morality in everyday interaction. Discourse Studies, 5(1), 79-100. https://doi.org/10.1177/14614456030050010401

Sterponi, L., \& de Kirby, K. (2016). A multidimensional reappraisal of language in autism: Insights from a discourse analytic study. Journal of Autism and Developmental Disorders, 46(2), 394-405. https://doi.org/10.1007/s10803-015-2679-z

Sterponi, L., de Kirby, K., \& Shankey, J. (2015). Rethinking language in autism. Autism, 19(5), 517-526. https://doi.org/10.1177/1362361314537125

Sterponi, L., \& Shankey, J. (2014). Rethinking echolalia: Repetition as interactional resource in the communication of a child with autism. Journal of Child Language, 41(02), 275304. https://doi.org/10.1017/S0305000912000682

Stevanovic, M. (2018). Social deontics: A nano-level approach to human power play. Journal for the Theory of Social Behaviour, 48(3), 369-389. https://doi.org/10.1111/jtsb.12175

Stickle, T., Duck, W., \& Maynard, D. W. (2017). Children's use of “I don't know” during clinical evaluations for autism spectrum disorder: Responses to emotion questions. In M. O'Reilly, J. N. Lester, \& T. Muskett (Eds.), A Practical Guide to Social Interaction Research in Autism Spectrum Disorders (pp. 247-273). Palgrave Macmillan UK. https://doi.org/10.1057/978-1-137-59236-1_10

Stivers, T., Sidnell, J., \& Bergen, C. (2018). Children's responses to questions in peer interaction: A window into the ontogenesis of interactional competence. Journal of Pragmatics, 124, 14-30. https://doi.org/10.1016/j.pragma.2017.11.013

Stokoe, E. (2012). Moving forward with membership categorization analysis: Methods for systematic analysis. Discourse Studies, 14(3), 277-303. https://doi.org/10.1177/1461445612441534

Stokoe, E. (2014). The Conversation Analytic Role-play Method (CARM): A Method for Training Communication Skills as an Alternative to Simulated Role-play. Research on Language and Social Interaction, 47(3), 255-265. https://doi.org/10.1080/08351813.2014.925663

Stribling, P., Rae, J., \& Dickerson, P. (2007). Two forms of spoken repetition in a girl with autism. International Journal of Language \& Communication Disorders, 42(4), 427444. https://doi.org/10.1080/13682820601183659

Stribling, P., Rae, J., \& Dickerson, P. (2009). Using conversation analysis to explore the recurrence of a topic in the talk of a boy with an autism spectrum disorder. Clinical Linguistics \& Phonetics, 23(8), 555-582. https://doi.org/10.1080/02699200802491165 
Surian, L., Baron-Cohen, S., \& Van der Lely, H. (1996). Are children with autism deaf to gricean maxims? Cognitive Neuropsychiatry, 1(1), 55-72. https://doi.org/10.1080/135468096396703

Tager-Flusberg, H., \& Anderson, M. (1991). The development of contingent discourse ability in autistic children. Journal of Child Psychology and Psychiatry, and Allied Disciplines, 32(7), 1123-1134.

Tager-Flusberg, H. (2009). Language and communication disorders in Autism Spectrum Disorders. In The neurobiology of autism (2nd ed., pp. 45-58). Johns Hopkins University Press.

Ten Have, P. (2002). The notion of member is the heart of the matter: On the role of membership knowledge in ethnomethodological inquiry. Forum Qualitative Sozialforschung / Forum: Qualitative Social Research, Vol 3. https://doi.org/10.17169/FQS-3.3.834

ten Have, P. (2004). Understanding qualitative research and ethnomethodology. SAGE Publications. https://ebookcentral.proquest.com

ten Have, P. (2007). Doing conversation analysis: A practical guide, 2nd ed. Sage Publications Ltd.

Thanem, T., \& Knights, D. (2019). Embodied research methods. SAGE Publications, Ltd. https://doi.org/10.4135/9781529716672

Thayer, S. (1986). History and strategies of research on social touch. Journal of Nonverbal Behavior, 10(1), 12-28. https://doi.org/10.1007/BF00987202

Torske, T., Nærland, T., Øie, M. G., Stenberg, N., \& Andreassen, O. A. (2018).

Metacognitive aspects of executive function are highly associated with social functioning on parent-rated measures in children with autism spectrum disorder. Frontiers in Behavioral Neuroscience, 11, 258. https://doi.org/10.3389/fnbeh.2017.00258

Turowetz, J. (2015). Citing conduct, individualizing symptoms: Accomplishing autism diagnosis in clinical case conferences. Social Science \& Medicine, 142, 214-222. https://doi.org/10.1016/j.socscimed.2015.08.022

Turowetz, J., \& Rawls, A. W. (2020). The development of Garfinkel's 'Trust' argument from 1947 to 1967: Demonstrating how inequality disrupts sense and self-making. Journal of Classical Sociology, 1468795X1989442. https://doi.org/10.1177/1468795X19894423 
van Heijst, B. F., \& Geurts, H. M. (2015). Quality of life in autism across the lifespan: A meta-analysis. Autism, 19(2), 158-167. https://doi.org/10.1177/1362361313517053

Veenstra-VanderWeele, J., Muller, C. L., Iwamoto, H., Sauer, J. E., Owens, W. A., Shah, C. R., Cohen, J., Mannangatti, P., Jessen, T., Thompson, B. J., Ye, R., Kerr, T. M., Carneiro, A. M., Crawley, J. N., Sanders-Bush, E., McMahon, D. G., Ramamoorthy, S., Daws, L. C., Sutcliffe, J. S., \& Blakely, R. D. (2012). Autism gene variant causes hyperserotonemia, serotonin receptor hypersensitivity, social impairment and repetitive behavior. Proceedings of the National Academy of Sciences, 109(14), 5469-5474. https://doi.org/10.1073/pnas.1112345109

Vom Lehn, D. (2014). Harold Garfinkel: The creation and development of ethnomethodology. Left Coast Press, Inc.

Vostanis, P., Smith, B., Corbett, J., Sungum-Paliwal, R., Edwards, A., Gingell, K., Golding, R., Moore, A., \& Wiilliams, J. (1998). Parental concerns of early development in children with autism and related disorders. Autism, 2(3), 229-242. https://doi.org/10.1177/1362361398023002

Walker, N. (2013). Throw away the master's tools: Liberating ourselves from the pathology paradigm. https://neurocosmopolitanism.com/throw-away-the-masters-tools-liberatingourselves-from-the-pathology-paradigm/

Walker, N. (2014). Neurodiversity: Some basic terms \& definitions. https://neurocosmopolitanism.com/neurodiversity-some-basic-terms-definitions/

Waltz, M. (2013). Autism a social and medical history. Palgrave Macmillan.

Waltz, M. (2014). Worlds of autism: Across the spectrum of neurological difference. Disability \& Society, 29(8), 1337-1338. https://doi.org/10.1080/09687599.2014.934064

Warnell, K. R., \& Redcay, E. (2019). Minimal coherence among varied theory of mind measures in childhood and adulthood. Cognition, 191, 103997. https://doi.org/10.1016/j.cognition.2019.06.009

Watson, D. R. (1978). Categorization, authorization and blame-Negotiation in conversation. Sociology, 12(1), 105-113. https://doi.org/10.1177/003803857801200106

Weatherall, A. (2011). I don't know as a prepositioned epistemic hedge. Research on Language \& Social Interaction, 44(4), 317-337. https://doi.org/10.1080/08351813.2011.619310 
Whitehead, K. A., Bowman, B., \& Raymond, G. (2018). "Risk factors" in action: The situated constitution of "risk" in violent interactions. Psychology of Violence, 8(3), 329-338. https://doi.org/10.1037/vio0000182

Whitney, D. G., \& Shapiro, D. N. (2019). National prevalence of pain among children and adolescents with autism spectrum disorders. JAMA Pediatrics, 173(12), 1203. https://doi.org/10.1001/jamapediatrics.2019.3826

Wiggins, S., \& Potter, J. (2003). Attitudes and evaluative practices: Category vs. item and subjective vs. objective constructions in everyday food assessments. British Journal of Social Psychology, 42(4), 513-531. https://doi.org/10.1348/014466603322595257

Wilkinson, K. M. (1998). Profiles of language and communication skills in autism. Mental Retardation and Developmental Disabilities Research Reviews, 4(2), 73-79. https://doi.org/10.1002/(SICI)1098-2779(1998)4:2<73::AID-MRDD3>3.0.CO;2-Y

Wilkinson, R. (2019). Atypical interaction: Conversation analysis and communicative impairments. Research on Language and Social Interaction, 52(3), 281-299. https://doi.org/10.1080/08351813.2019.1631045

Wilkinson, R., Rae, J. P., \& Rasmussen, G. (Eds.). (2020). Atypical interaction: The impact of communicative impairments within everyday talk. Springer International Publishing. https://doi.org/10.1007/978-3-030-28799-3

Williams, V. (2011). Disability and discourse: Analysing inclusive conversation with people with intellectual disabilities. John Wiley \& Sons, Ltd. https://doi.org/10.1002/9780470977934

Williams, V., Webb, J., Read, S., James, R., \& Davis, H. (2020). Future lived experience: Inclusive research with people living with dementia. Qualitative Research, 20(5), 721740. https://doi.org/10.1177/1468794119893608

Willis, F. N., \& Briggs, L. F. (1992). Relationship and touch in public settings. Journal of Nonverbal Behavior, 16(1), 55-63. https://doi.org/10.1007/BF00986879

Willis, F. N., \& Rinck, C. M. (1983). A personal log method for investigating interpersonal touch. The Journal of Psychology, 113(1), 119-122. https://doi.org/10.1080/00223980.1983.9923565

Wing, L. (1981a). Asperger's syndrome: A clinical account. Psychological Medicine, 11(01), 115. https://doi.org/10.1017/S0033291700053332

Wing, L. (1981b). Language, social, and cognitive impairments in autism and severe mental retardation. Journal of Autism and Developmental Disorders, 11(1), 31-44. 
Wing, L., \& Gould, J. (1979). Severe impairments of social interaction and associated abnormalities in children: Epidemiology and classification. Journal of Autism and Developmental Disorders, 9(1), 11-29.

Wing, L., Gould, J., Yeates, S. R., \& Brierly, L. M. (1977). Symbolic play in severely mentally retarded and in autistic children. Journal of Child Psychology and Psychiatry, 18(2), 167-178. https://doi.org/10.1111/j.1469-7610.1977.tb00426.x

Wittgenstein, L. (1958). Philosophical investigations (G. E. M. Anscombe, Trans.; 2nd ed.). Basil Blackwell.

Wood, C., \& Freeth, M. (2016). Students' stereotypes of autism. Journal of Educational Issues, 2(2), 131. https://doi.org/10.5296/jei.v2i2.9975

Woods, R., Milton, D., Arnold, L., \& Graby, S. (2018). Redefining Critical Autism Studies: A more inclusive interpretation. Disability \& Society, 33(6), 974-979. https://doi.org/10.1080/09687599.2018.1454380

Wootton, A. J. (2006). Children's practices and their connections with "mind." Discourse Studies, 8(1), 191-198. https://doi.org/10.1177/1461445606059567

Wootton, Anthony J. (1981). Two request forms of four year olds. Journal of Pragmatics, 5(6), 511-523. https://doi.org/10.1016/0378-2166(81)90016-3

Wootton, Anthony J. (1999). An investigation of delayed echoing in a child with autism. First Language, 19(57), 359-381. https://doi.org/10.1177/014272379901905704

Wootton, Anthony J. (2007). A puzzle about please: repair, increments, and related matters in the speech of a young child. Research on Language and Social Interaction, 40(2-3), 171-198. https://doi.org/10.1080/08351810701354623 


\section{Appendix A \\ Transcription Conventions}

The following describes the transcription conventions employed in this thesis. The basic conventions for the transcription of talk will be provided first in a table before the conventions for multimodal transcription of embodied activity. The conventions used here are derived from Hepburn and Bolden (2017) and Mondada (2016).

\begin{tabular}{|c|c|}
\hline Symbol & Description \\
\hline [word] & Onset and offset of overlapping speech \\
\hline$=$ & $\begin{array}{l}\text { Latched speech (continuing speech with no } \\
\text { pause between) }\end{array}$ \\
\hline word. & Turn-final, downward intonation \\
\hline word, & Turn-final, slight rising intonation \\
\hline word? & Turn-final, strong rising intonation \\
\hline word_ & Turn-final, stable intonation \\
\hline (.) & $\begin{array}{l}\text { Micropause (silence of less than one tenth } \\
\text { or a second) }\end{array}$ \\
\hline$(1.2)$ & Silences in seconds and tenths of seconds \\
\hline Word & Increased stress \\
\hline$\overline{\text { WORD }}$ & Increased volume \\
\hline Wo:rd & Sound elongation \\
\hline wor- & Sound cut off \\
\hline${ }^{\circ}$ word ${ }^{\circ}$ & Decreased volume \\
\hline $\mathrm{h}$ & Outbreath \\
\hline.$h$ & Inbreath \\
\hline$(($ word $))$ & Transcriber's commentary \\
\hline (word) & Possible/unclear hearing \\
\hline$>$ word $<$ & Quicker/temporally compressed speech \\
\hline${ }^{\wedge}$ word $^{\wedge}{ }^{\wedge}$ word $^{\wedge}$ & $\begin{array}{l}\text { Sharp increase in pitch returning to normal } \\
\text { through production/Sustained sharp increase } \\
\text { in pitch }\end{array}$ \\
\hline |word/|word| & $\begin{array}{l}\text { Sharp decrease in pitch returning to normal } \\
\text { through production/Sustained sharp } \\
\text { decrease in pitch }\end{array}$ \\
\hline \#word & Creaky voice (vocal fry) \\
\hline
\end{tabular}

Transcription of multimodal conduct follows Mondada's (2016) conventions.

Embodied conduct is described on its own lines beneath the co-occurring talk or silences. These lines are not numbered and producer of the embodied conduct is only marked when they are not the speaker of the co-occurring talk or when the action occurs within a period of silence. Onset and offset of embodied conduct is marked by a symbol (for example + or *) corresponding to the actor performing it. This symbol is marked on the line of talk during which the embodied conduct happens and on the following line where the conduct is 
described. When conduct continues across multiple lines, the ---> arrow is used to show this continuation until the corresponding conduct offset symbol. The temporal location of figures is marked on the lines of talk and in conduct lines with a \# and the number of the figure.

Some descriptions will include abbreviations such as RH or RA for right hand or right arm. An annotated example of a multimodal transcript is provided below.

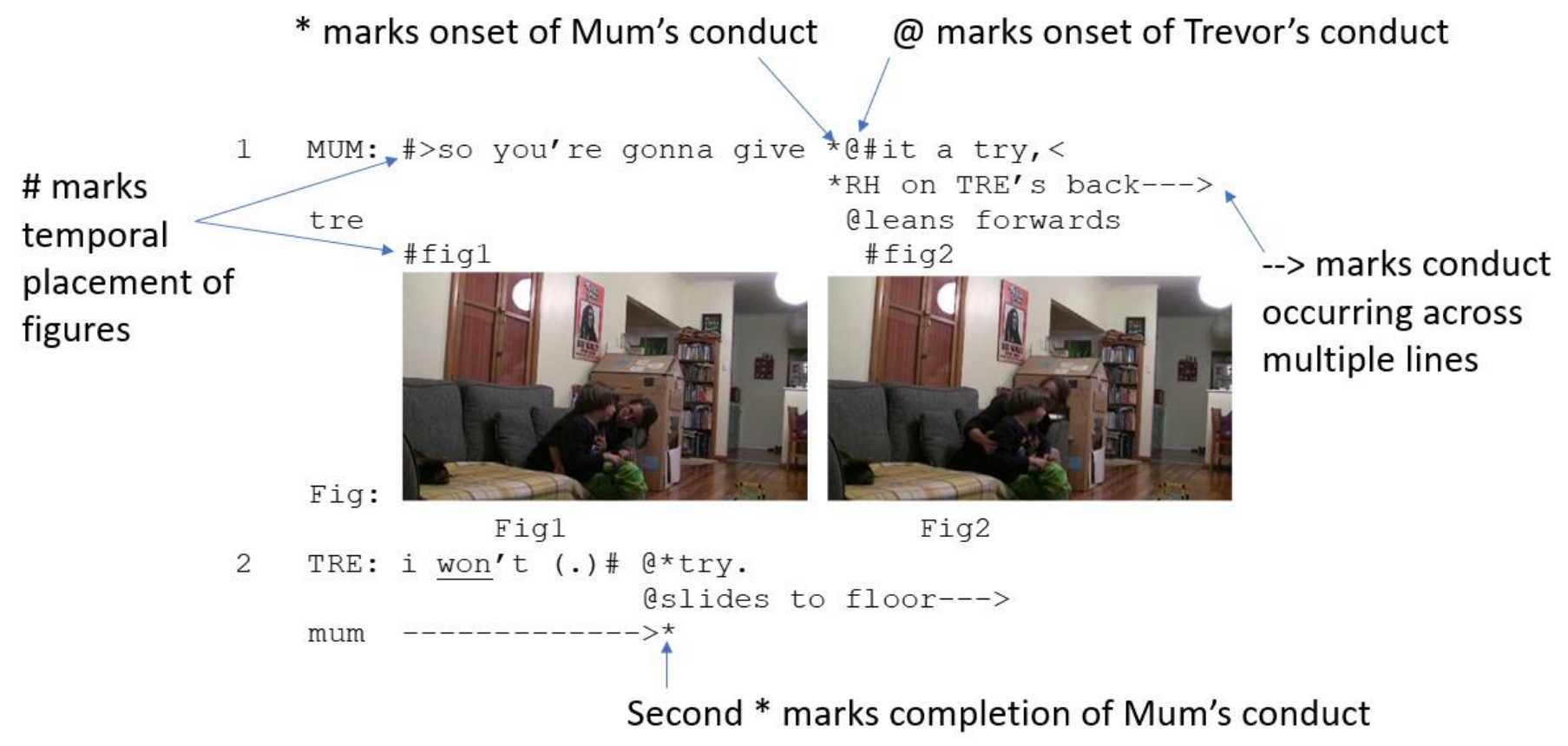

